\title{
The role of estrogen receptors in prostate cancer development and their contributions in new treatment opportunities
}

\section{Doctoral Thesis}

In partial fulfillment of the requirements for the degree

\author{
"Doctor rerum naturalium (Dr. rer. nat.)" \\ in the Molecular Medicine Study Program \\ at the Georg-August University Göttingen
}

submitted by

Julia Gehrig, née Meyer

born in Hamelin, Germany

Göttingen, 2016 


\section{Members of the Thesis Committee:}

\section{Official Supervisor:}

Prof. Dr. Peter Burfeind, Department of Human Genetics, University Medical Center Göttingen

Second member of the thesis committee:

Prof. Dr. Hubertus Jarry, Clinical and Experimental Endocrinology, University Medical Center Göttingen, Germany

Third member of the thesis committee:

Prof. Dr. Dieter Kube, Department of Hematology and Oncology, University Medical Center Göttingen

Date of Disputation: 


\section{AFFIDAVIT}

Herewith I declare that my doctoral thesis entitled "The role of estrogen receptors in prostate cancer development and their contributions in new treatment opportunities" has been written independently with no other sources and aids than quoted.

Göttingen, December 2016

Julia Gehrig 


\section{Table of contents}

Table of contents

Abbreviations

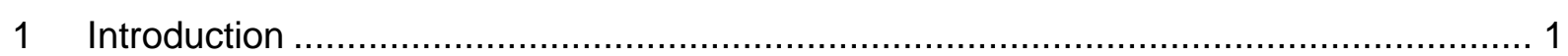

1.1 Prostate cancer- incidence, risk factors and treatment ...................................... 1

1.2 AR-targeted PCa treatment strategies and why they fail..................................... 2

1.3 New treatment strategies for prostate cancer ................................................ 4

1.3.1 ER $\beta$ as a potential target in PCa treatment ............................................ 5

1.3.1.1 Estrogen receptors- structure and signaling ...................................... 5

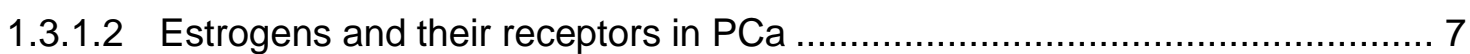

1.3.2 Amygdalin as a potential drug in PCa treatment.......................................... 9

1.3.2.1 The active agent amygdalin .................................................................... 9

1.3.2.2 Mechanism of metabolism of amygdalin ................................................10

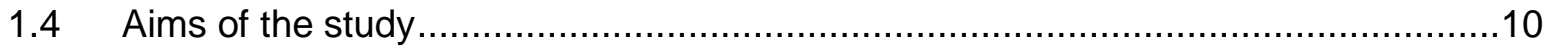

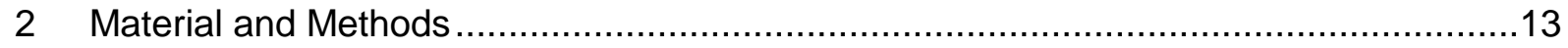

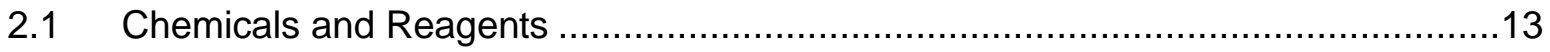

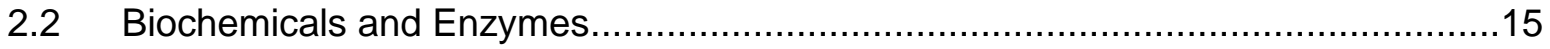

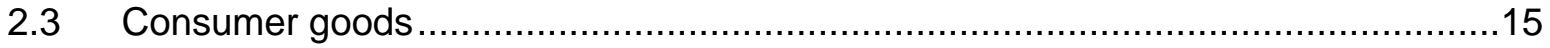

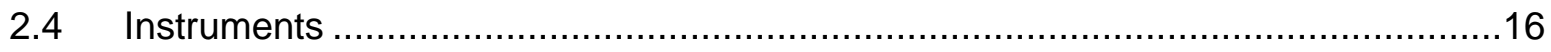

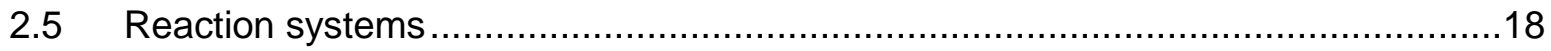

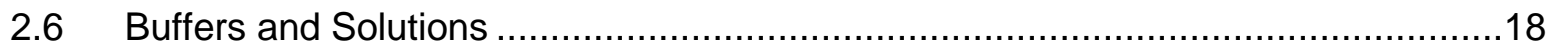

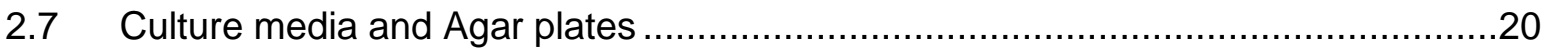

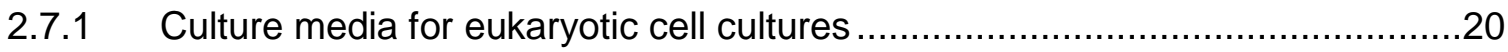

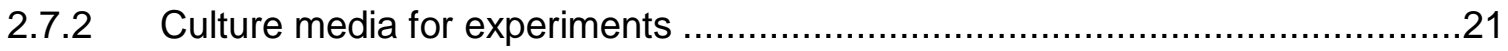

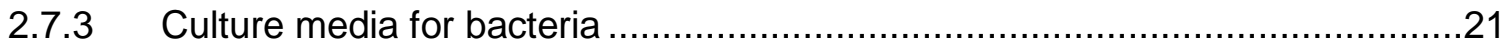

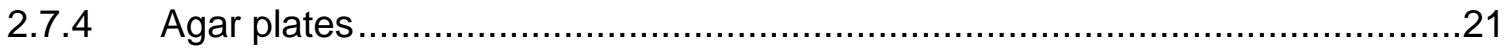

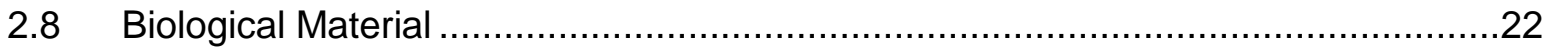

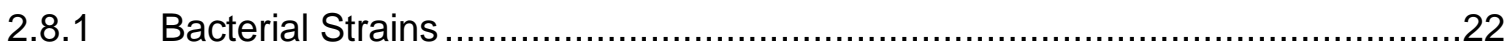

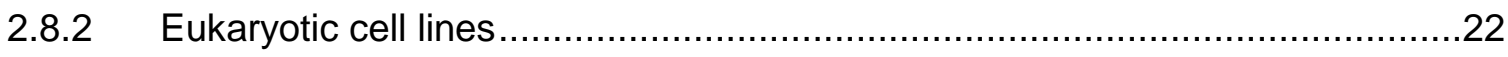

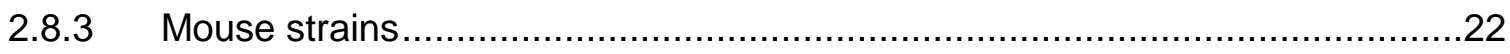

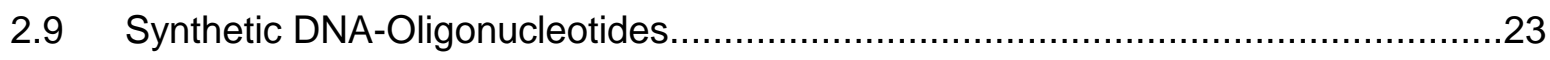

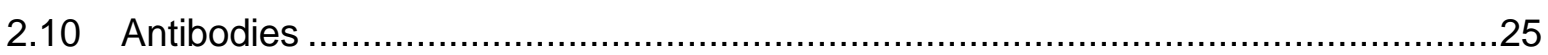

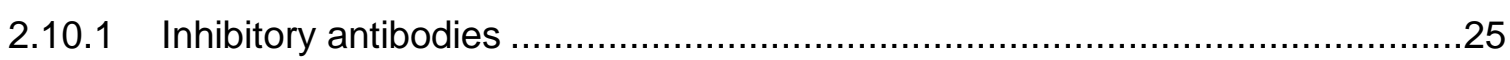

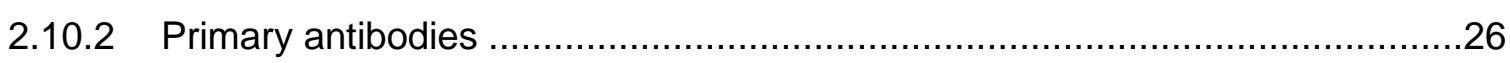

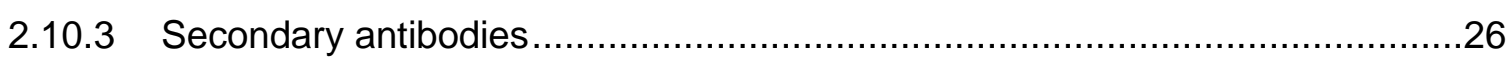

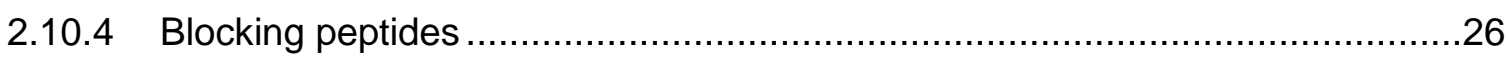




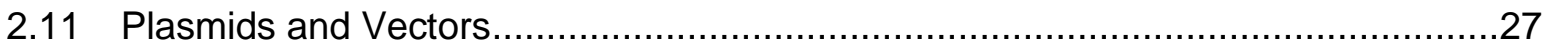

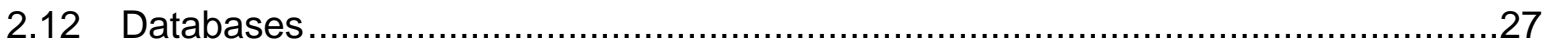

2.13 Isolation, purification and concentration measurement of nucleic acids ................27

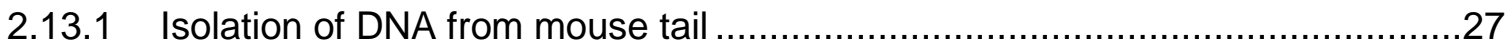

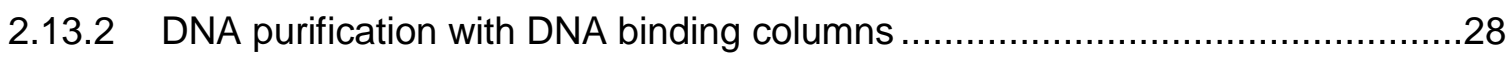

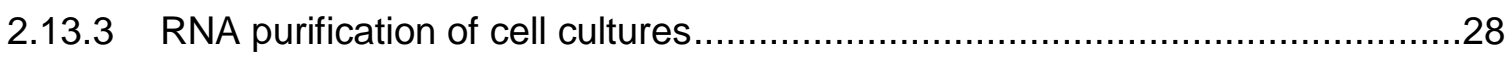

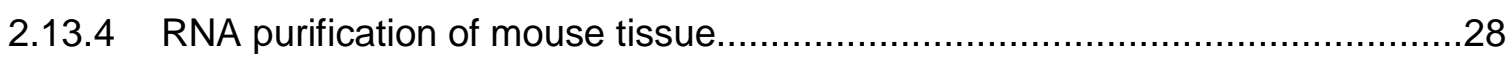

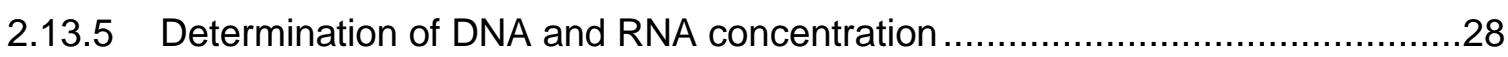

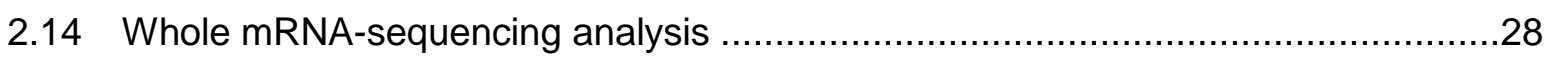

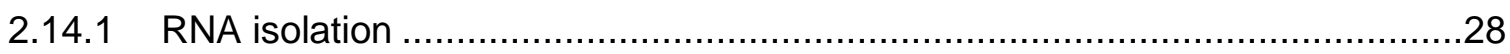

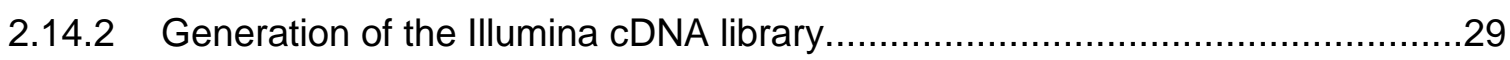

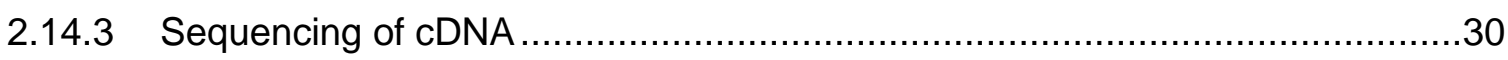

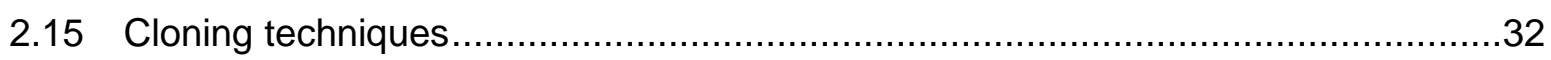

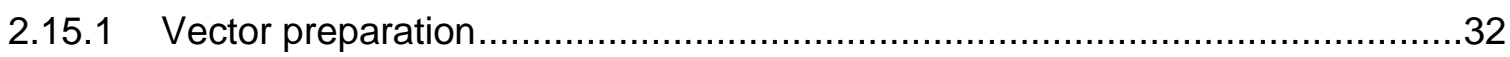

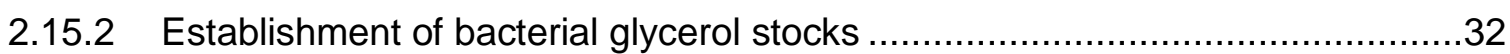

2.15.3 Transformation of DH5 $\alpha$ Escherichia coli bacteria ...........................................32

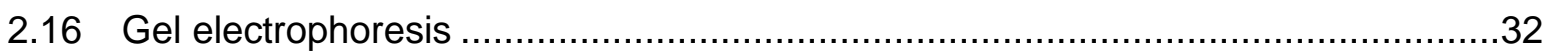

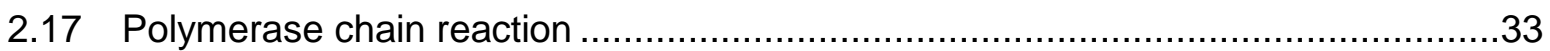

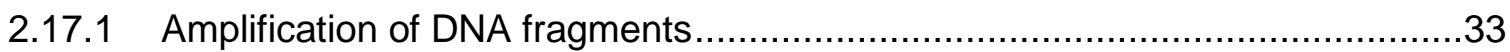

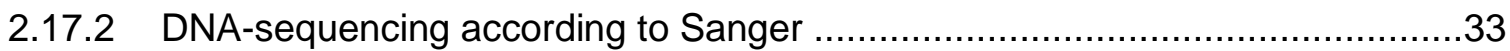

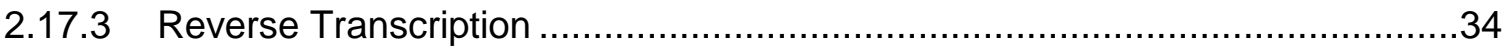

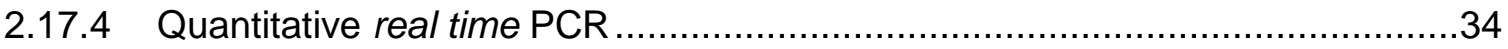

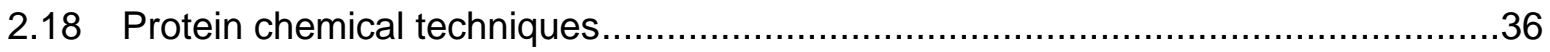

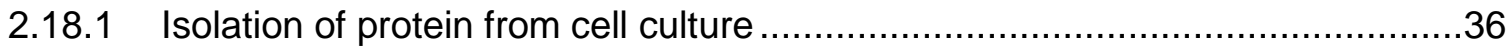

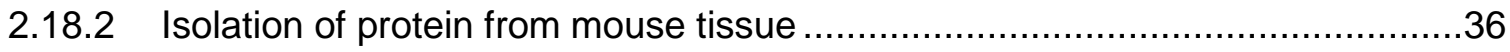

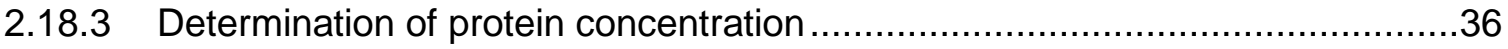

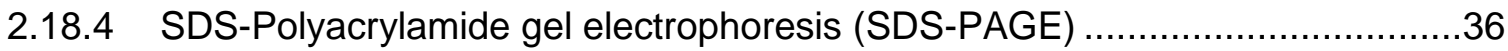

2.18.5 Western blotting of proteins onto Polyvinylidene Difluoride membranes ..........37

2.18.6 Incubation of protein-bound membranes with antibodies ...............................37

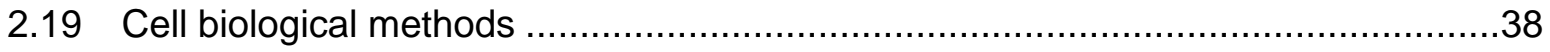

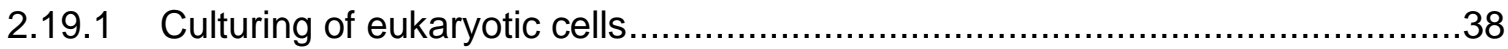

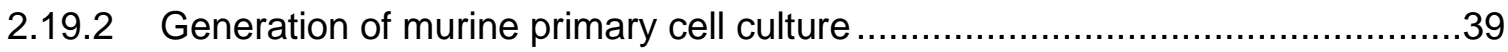

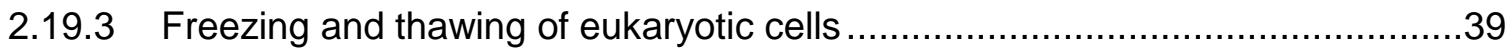

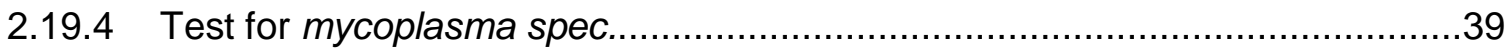

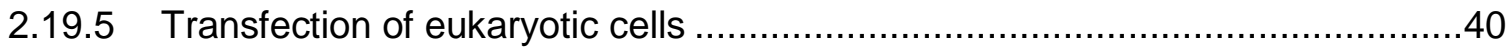

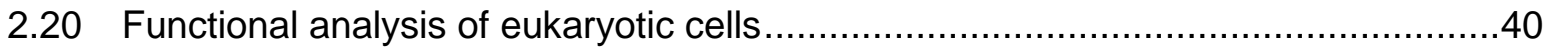

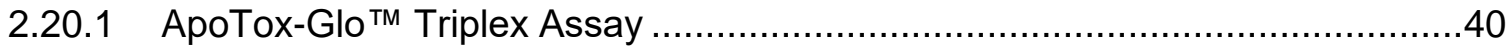




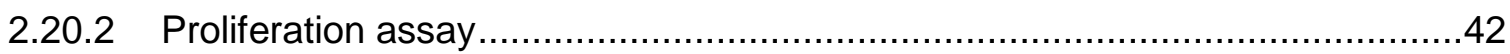

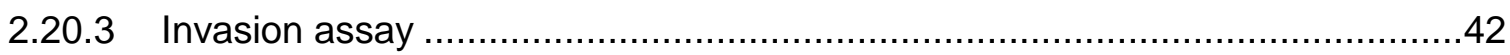

2.20.4 Senescence-associated beta-galactosidase staining..................................42

2.21 Dual-Luciferase Reporter Assay .................................................................43

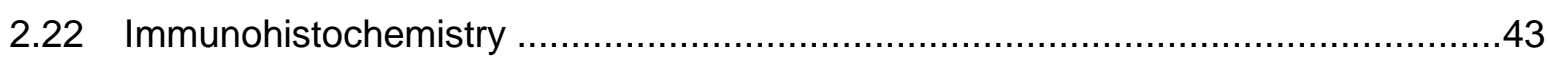

2.22.1 Immunofluorescence staining of eukaryotic cells ........................................43

2.22.2 Immunohistochemical staining of paraffin embedded tissue sections ..............44

2.23 Necrotic area calculation ............................................................................45

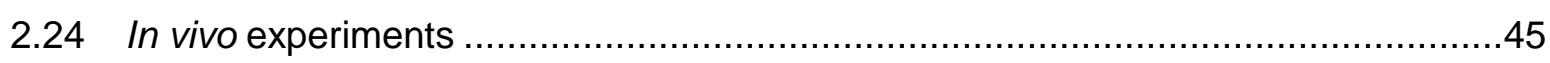

2.24.1 Treatment of TRAMP-FVB mice with amygdalin.........................................45

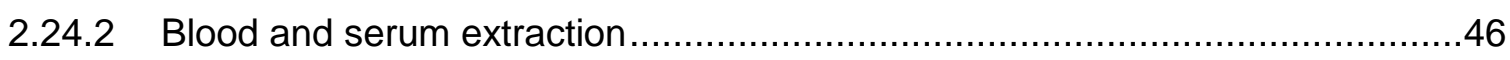

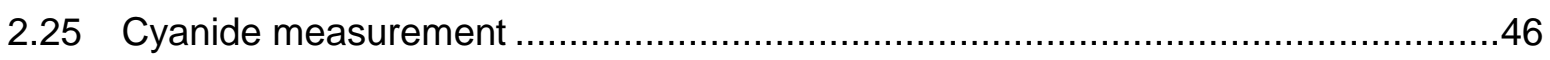

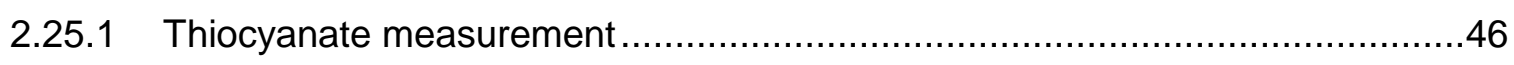

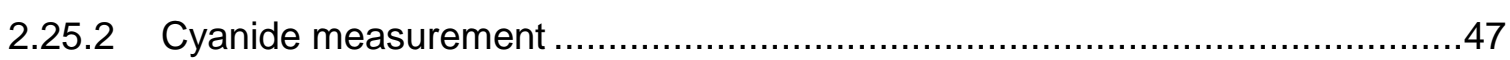

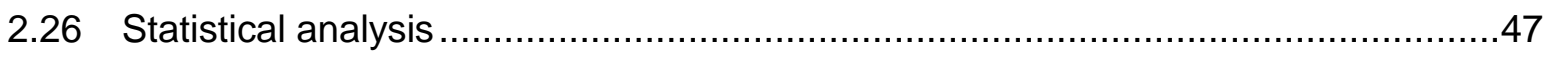

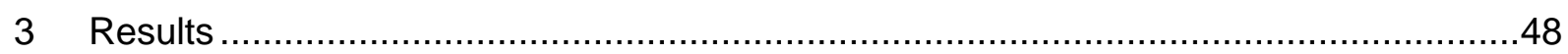

3.1 Investigation of the role of estrogen receptors in prostate cancer .........................48

3.1.1 Analysis of the role of ER $\alpha$ and ER $\beta$ knockout in the TRAMP mouse model .48

3.1.1.1 Survival times of ERKO, BERKO, TRAMP, ERKO/TRAMP and BERKO/TRAMP mice

3.1.1.2 Histopathological analysis of genotype-specific abnormalities in organ formation

3.1.1.3 Histopathologic analysis of the urogenital tract in TRAMP, ERKO/TRAMP and BERKO/TRAMP mice

3.1.1.4 Establishment of PCa cell lines from TRAMP and BERKO/TRAMP mice ....52

3.1.1.5 Confirmation of the Esr2 knockout genotype in BERKO/TRAMP 283 cells ..52

3.1.1.6 Expression of ER $\beta$ in BERKO/TRAMP 283 and T244 cells. .54

3.1.1.7 Sequencing of exon 3 of the Esr2 gene to confirm the insertion of a stop codon......

3.1.1.8 Analysis of the proliferation rate of T244 and BERKO/TRAMP 283 cells .....56

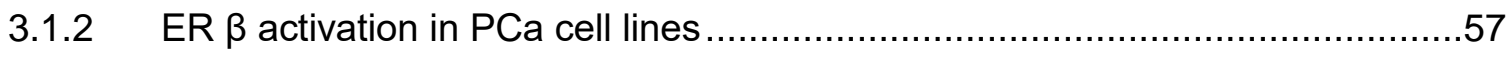

3.1.2.1 Identification of differentially expressed genes in VCaP rev., VCaP and VCaP AA cells .57

3.1.2.2 Analysis of the effect of $8 \beta-\mathrm{VE} 2$ treatment in VCaP cell variants .58

3.1.2.3 Whole mRNA-sequencing analysis in VCaP cell variants after $8 \beta$-VE2 treatment

3.1.2.4 Analysis of the role of ER $\beta$ in 8$\beta$-VE2-mediated treatment effects

3.1.2.4.1 Analyses of cellular effects of ERB 041 treatment on VCaP cell variants69 
3.1.2.4.2 Analyses of cellular effects of FERb 033 treatment on $\mathrm{VCaP}$ cell variants.

3.1.2.5 Analyses of alternative ER $\beta$-mediated signaling pathways .76

3.1.2.6 Analysis of the role of the cholesterol pathway in 8ß-VE2-mediated treatment effects. .78

3.1.2.7 Analysis of the role of PI3K and MAPK signaling pathways in 8 $\beta$-VE2mediated treatment effects

3.1.2.7.1 Analyses of PI3K and MAPK pathway inhibition as the underlying mechanism for $8 \beta$-VE2-induced effects on VCaP cells.

3.1.2.7.2 Analysis of PI3K as a mediator of 8ß-VE2-induced effects in VCaP cells..

3.1.2.8 Investigation of underlying mechanism of AR target gene expression after $8 \beta$-VE2 treatment

3.1.2.8.1 Analysis of glucocorticoid receptor expression and transcriptional activity in VCaP cells .86

3.1.2.8.2 The role of AR in 8 $\beta$-VE2-mediated treatment effects .87

3.1.2.8.2.1 Analysis of $A R$ and $A R$ target gene expression after inhibition of testosterone or AR signaling in VCaP cell variants.

3.1.2.8.2.2 Analysis of the effect of DHT treatment in VCaP cells....

3.1.2.8.2.3 Analysis of the effect of $8 \beta-V E 2$ on the human PCa cell line PC3 ....92

3.1.2.8.2.4 Analysis of AR activation after 8 $\beta$-VE2 treatment using a reporter gene assay in VCaP cells

3.2 Influence of amygdalin on mouse prostate cancer .95

3.2.1 Amygdalin treatment of TRAMP-FVB mice .95

3.2.1.1 Analysis of side effects in TRAMP-FVB mice associated with amygdalin treatment

3.2.1.2 Impact of amygdalin treatment on overall survival time of TRAMP-FVB mice.

3.2.1.3 Tumor burden and metastasis formation in amygdalin-treated TRAMP-FVB mice

3.2.1.4 Examination of the mechanism of amygdalin metabolism. 102

3.2.1.4.1 Analysis of the expression of $\beta$-glucosidase and rhodanase in mouse tissues.

3.2.1.4.2 Detection of cyanide $\left(\mathrm{CN}^{-}\right)$and thiocyanate $\left(\mathrm{SCN}^{-}\right)$in blood samples of amygdalin-treated TRAMP-FVB mice.

3.2.1.5 Correlation of amygdalin treatment and the formation of necrotic tumor areas in TRAMP-FVB mice.

3.2.2 In vitro analyses of the effect of amygdalin on murine PCa cells 109

3.2.2.1 Effect of amygdalin on cell survival, cytotoxicity and apoptosis induction on T244 and 2E cells 
3.2.2.2 Analysis of protein integrity of amygdalin-treated primary PCa cell lines T244 and $2 \mathrm{E}$

3.2.2.3 Effect of amygdalin on the invasive potential of primary PCa cell lines T244 and $2 \mathrm{E}$.

3.2.2.4 Morphological analysis of amygdalin-treated T244 and 2E cells 112

3.2.2.5 Analysis of cell number in amygdalin-treated primary PCa cell lines T244 and $2 \mathrm{E}$.

3.2.2.6 Analysis of proliferation in amygdalin-treated primary PCa cell lines T244 and $2 \mathrm{E}$.

3.2.2.7 Analysis of senescence induction in amygdalin-treated primary PCa cell lines T244 and 2E

3.2.2.8 Analysis of senescence inducing factors in amygdalin-treated primary PCa cell lines T244 and 2E

3.2.2.8.1 Detection of $\mathrm{yH} 2 \mathrm{Ax}$ expression in amygdalin-treated primary $\mathrm{PCa}$ cell lines T244 and 2E

3.2.2.8.2 Detection of mitotic catastrophe in amygdalin-treated primary PCa cell lines T244 and 2E

4 Discussion

4.1 Summary of results 123

4.2 Are the used ER mouse models suitable for knockout experiments? 125

4.3 Treatment of PCa cells with the ER $\beta$ agonist $8 \beta-V E 2$ 130

4.3.1 Are the 8$\beta$-VE2-induced effects dependent on ER $\beta$ signaling? 135

4.3.2 Are the 8$\beta$-VE2-induced effects in VCaP cells dependent on the cholesterol synthesis pathway?

4.3.3 Are the 8ß-VE2-induced effects in VCaP cells dependent on PI3K signaling?....

4.3.4 Are the 8ß-VE2-induced effects in VCaP cells dependent on AR signaling? .139

4.4 AR-mediated alternative survival pathways .................................................. 145

4.5 Amygdalin- a potent anti-cancer agent or toxic nonsense? ................................149

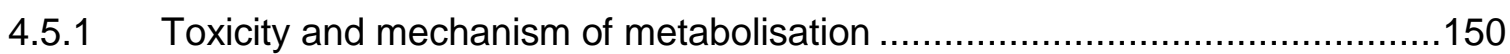

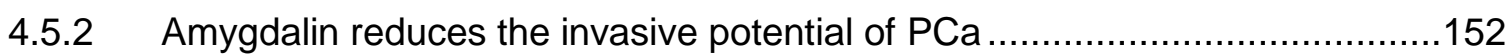

4.5.3 Amygdalin treatment induces senescence in vitro .......................................152

4.5.4 Amygdalin treatment induces necrosis in vivo ............................................155

4.5.5 How could amygdalin mediates its anti-cancerous effects? ..........................156

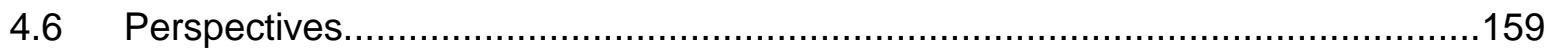

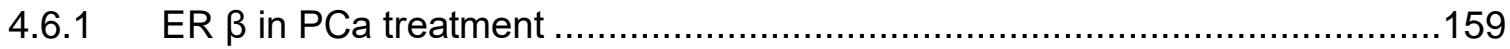

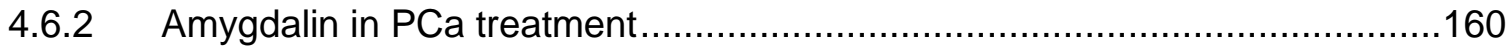

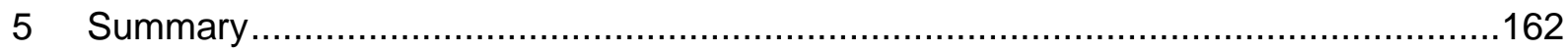

5.1 The role of ER $\beta$ and effects of $8 \beta$-VE2 treatment in PCa................................162 


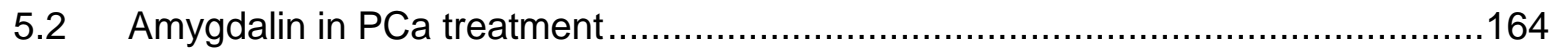

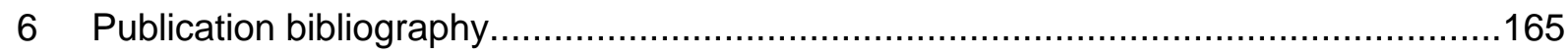

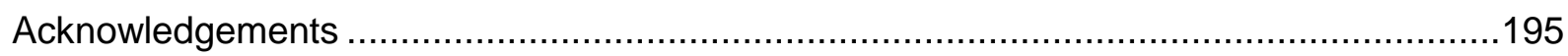




\section{Abbreviations}

8ß-VE2

$\alpha$

A

AA

ADP

ADT

Adv

AED

AGE

AMP

AR

ARV

ATP

Bax

BERKO

Bid

bis-AAF-R110

bp

BSA

${ }^{\circ} \mathrm{C}$

C

$\mathrm{Ca}^{2+}$

cDNA

$\mathrm{CN}^{-}$

$\mathrm{CO}_{2}$

CPM

CRPC

Ct value

CYP17

DAPI

DBD

$\mathrm{ddH}_{2} \mathrm{O}$

DHCR

DHT

DMSO

DNA
8-Vinylestra-1,3,5 (10)-triene-3,17ß-diol

Anti

Purine base Adenin

Abiraterone acetate resistant

Adenosine diphosphate

Androgen deprivation therapy

Advanced

5'-Androstenediol

Aminoglutethimide

Adenosine monophosphate

Androgen receptor

Androgen receptor splice variant

Adenosine triphosphate

B-cell lymphoma 2-associated $\mathrm{X}$

Beta estrogen receptor knockout

$\mathrm{BH} 3$ interacting-domain death agonist

Bis-alanylalanyl-phenylalanyl-rhodamine 110

Base pair

Bovine serum albumin

Degree Celsius

Pyrimidine base Cytosine

Calcium

Complementary DNA

Cyanide

Carbon dioxide

Counts per million

Castration-resistant prostate cancer

Threshold value

17a-Hydroxylase

4',6-Diamidino-2-phenylindole dihydrochloride

DNA binding domain

Bi-distilled $\mathrm{H}_{2} \mathrm{O}$

Dehydrocholesterol reductase

Dihydrotestosterone

Dimethyl sulfoxide

Deoxyribonucleic acid 
dNTP

DSB

DTT

E2

ECL

EDTA

e.g.

EGF

EGFR

EMT

ER

ERB 041

ERKO

et al.

FBS

FDA

FDR

FDPS

FERb 033

FGF

FITC

Fw

g

G

GF-AFC

GR

$\mathrm{h}$

$\mathrm{H}_{2} \mathrm{O}_{2}$

HBSS

HDR

HE3235

HMGCR

HRP

ICC

IDI1

i.e.

$\lg G$
Deoxyribonucleotide phosphate

Double strand break

1,4-Dithiothreitol

Estradiol

Enhanced chemical luminescence

Ethylenediamine tetraacetic acid

exempli gratia (for example)

Epidermal growth factor

Epidermal growth factor receptor

Epithelial-mesenchymal transition

Estrogen receptor

7-Ethenyl-2-(3-fluoro-4-hydroxyphenyl)-5-benzoxazolol

Alpha estrogen receptor knockout

et alteri (and others)

Fetal bovine serum

Food and drug administration

False discovery rate

Farnesyl diphosphate synthase

2-Chloro-3'-fluoro-3,4'-dihydroxy-[1,1-biphenyl]-4-

carboxaldehyde oxime

Fibroblast growth factor

Fluorescein isothiocyanate

Forward

Gram

Purine base Guanine

Glycyl-phenylalanyl-aminofluorocumarin

Glucocorticoid receptor

Hour

Hydrogen peroxide

Hank's Balanced Salt Solution

High dose rate

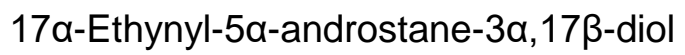

3-Hydroxy-3-methylglutaryl-CoA reductase

Horseradish peroxidise

Immunocytochemistry

Isopentenyl-diphosphate delta isomerase 1

id est (that is)

Immunoglobulin G 


\begin{tabular}{|c|c|}
\hline $\mathrm{IHC}$ & Immunohistochemistry \\
\hline $\mathrm{kDa}$ & Kilodalton \\
\hline LBD & Ligand binding domain \\
\hline $\mathrm{LHRH}$ & Luteinizing hormone-releasing hormone \\
\hline Luc & Luciferase \\
\hline M & Molar (moles per litre) \\
\hline $\mathrm{mA}$ & Milliampere \\
\hline MAPK & Mitogen-activated protein kinase \\
\hline MDC & Moderate-differentiated carcinoma \\
\hline MDM2 & Mouse double minute 2 \\
\hline $\mathrm{MeOH}$ & Methanol \\
\hline $\mathrm{Mg}^{2+}$ & Magnesium \\
\hline $\mathrm{mg}$ & Milligram \\
\hline $\min$ & Minute \\
\hline $\mathrm{mM}$ & Millimolar \\
\hline MTS & $\begin{array}{l}\text { 3-(4,5-Dimethylthiazol-2-yl)-5-(3-carboxymethoxyphenyl)-2-(4- } \\
\text { sulfophenyl)-2H-tetrazolium }\end{array}$ \\
\hline $\mathrm{NaCl}$ & Sodium chloride \\
\hline $\mathrm{NaOH}$ & Sodium hydroxide \\
\hline $\mathrm{NCBI}$ & National Center for Biotechnology Information \\
\hline $\mathrm{ng}$ & Nanogram \\
\hline $\mathrm{nm}$ & Nanometer \\
\hline $\mathrm{nM}$ & Nanomolar \\
\hline NR & Nuclear receptor \\
\hline $\mathrm{O}_{2}$ & Oxygen \\
\hline P450scc & Cholesterol side chain cleavage enzyme \\
\hline PAGE & Polyacrylamide gel electrophoresis \\
\hline PARP & Poly (ADP-ribose) polymerase \\
\hline PBS & Phosphate buffered saline \\
\hline $\mathrm{PCa}$ & Prostate cancer \\
\hline PCA3 & Prostate cancer associated 3 \\
\hline PCR & Polymerase chain reaction \\
\hline PDC & Poorly-differentiated PCa \\
\hline PFBBr & 2,3,4,5,6-Pentafluorobenzylbromid \\
\hline PFBSCN & 2,3,4,5,6-Pentafluorobenzylthiocyanat \\
\hline $\mathrm{pH}$ & Preponderance of hydrogen ions \\
\hline PI3K & Phosphatidylinositol-4,5-bisphosphate 3-kinase \\
\hline PIN & Prostatic intraepithelial neoplasia \\
\hline
\end{tabular}




\begin{tabular}{|c|c|}
\hline PML & Promyelocytic leukemia protein \\
\hline pmol & Picomolar \\
\hline PMS & Phenazine methosulfate \\
\hline PMSF & Phenylmethylsulfonyl fluoride \\
\hline$P P_{i}$ & Pyrophosphat \\
\hline $\mathrm{pRB}$ & Retinoblastoma protein \\
\hline $\mathrm{P} / \mathrm{S}$ & Penicillin/streptomycin \\
\hline PSA & Prostate-specific antigen \\
\hline PUMA & p53 upregulated modulator of apoptosis \\
\hline PVDF & Polyvinylidene Difluoride \\
\hline qRT-PCR & Quantitative real time PCR \\
\hline $\operatorname{Rev}$ & Reverse \\
\hline RIP1 & Receptor-interacting protein \\
\hline RKI & Robert Koch Institute \\
\hline RNA & Ribonucleic acid \\
\hline RNase & Ribonuclease \\
\hline ROS & Reactive oxygen species \\
\hline RT & Room temperature \\
\hline SA $\beta$-gal & Senescence associated $\beta$-galactosidase \\
\hline $\mathrm{SCN}^{-}$ & Thiocyanate \\
\hline SDS & Sodium dodecyl sulfate \\
\hline SDS-PAGE & SDS-polyacrylamide gel electrophoresis \\
\hline sec & Second \\
\hline SGCa & Seminal gland cancer \\
\hline spec. & Species \\
\hline SV 40 & Simian virus 40 \\
\hline $\mathrm{T}$ & Pyrimidine base Thymidine \\
\hline TBS & Tris-buffered saline \\
\hline TMPRSS2-ERG & $\begin{array}{l}\text { Transmembrane protease, serine 2- erythroblast } \\
\text { transformation-specific-related gene }\end{array}$ \\
\hline TNFR1 & Tumor necrosis factor receptor 1 \\
\hline TRAMP & Transgenic adenocarcinoma of mouse prostate \\
\hline Tris & Trihydroxymethylaminomethane \\
\hline$U$ & Units \\
\hline UV & Ultraviolet \\
\hline V & Voltage \\
\hline VCaP & Vertebral-Cancer of the Prostate \\
\hline VEGF & Vascular endothelial growth factor \\
\hline
\end{tabular}


Vol

WDC

$x g$

$X-G a l$

$\mu \mathrm{g}$

$\mu l$

$\mu \mathrm{M}$
Volume

Well-differentiated PCa

Multiple of acceleration of gravity

5-Bromo-4-chloro-3-indolyl-a-D-galactopyranoside

Microgram

Microlitre

Micromolar 


\section{Introduction}

\subsection{Prostate cancer- incidence, risk factors and treatment}

Prostate cancer (PCa) is the second most common cancer type worldwide, representing $15 \%$ of all diagnosed cancers in men (World Cancer Research Fund, 2012). In Germany, $\mathrm{PCa}$ is the most common newly diagnosed cancer type in men with more than 60.000 diagnosed cases every year and the third leading reason for cancer related death (RobertKoch-Institut, Gesellschaft der epidemiologischen Krebsregister in Deutschland 2015). PCa mostly occurs in men older than 50 years. Primary PCa progresses slowly and the 5-year survival rate is high (93\%) (Robert-Koch-Institut, Gesellschaft der epidemiologischen Krebsregister in Deutschland 2015). Screening for PCa is performed by digital rectal examination and measurement of prostate-specific antigen (PSA). PSA blood concentrations $>4 \mathrm{ng} / \mathrm{ml}$ need further examination. However, disadvantage of PSA as a marker is a relatively high detection of false positive results and for diagnosis confirmation tissue biopsies are necessary. Currently, other marker genes, e.g. the fusion gene TMPRSS2-ERG (Transmembrane protease, serine 2- erythroblast transformation-specific-related gene) or PCA3 (prostate cancer associated 3) are under investigation (Barry Delongchamps 2014), to improve non-invasive PCa diagnostics.

Little is known about risk factors for PCa. Certainly, the risk of disease increases with age. The Robert-Koch-Institute stated that the likelihood to develop PCa within the next 10 years is $0.1 \%$ for 35 -year-old men; for 70 -year-old men the risk is $6 \%$. Clustering between near relatives indicate an involvement of hereditary factors, however no gene mutation could be connected to PCa development to date (Robert-Koch-Institut, Gesellschaft der epidemiologischen Krebsregister in Deutschland 2015). Also environmental factors seem to have influence on PCa risk. For example, diet is supposed to be an important factor. Whereas high fat diet might facilitate PCa development (Sonn et al. 2005), the lower PCa incidence in Asian population seems to be reasoned in phytoestrogen-rich diet (Tominaga 1985; Goetzl et al. 2007). Hormone expression, in particular a high testosterone level, is reckoned to promote PCa development (Michaud et al. 2015).

The treatment of PCa distinguishes between localized and metastasized cancer. Localized $\mathrm{PCa}$ is commonly treated by either active surveillance or radical prostatectomy combined with irradiation. Also high dose rate (HDR)-brachytherapy is applied (S3 Leitlinie Prostatakarzinom - Deutsche Gesellschaft für Urologie, 2011). HDR-brachytherapy is an internal radiation by implanting weakly radioactive iodide into PCa tissue. This ensures long term treatment directly at the tumor tissue with minimal side effects (Tselis et al. 2013). Also other minimal invasive treatment approaches such as high intensity focal ultrasound, cryotherapy and laser phototherapy can be used for prostate cancer treatment (American cancer society, 2016; Zhu et al. 2005; Trachtenberg et al. 2007). Patients under active 
surveillance are under intense observation, but the cancer is not treated. This method is applicable in cases of slow growing carcinomas or aged patients when benefits of cancer treatment are small compared to the severe side effects induced by other treatment options. After radical prostatectomy and irradiation patients often suffer from incontinence and loss of potency, but for fast growing carcinomas it is still the method of choice to prevent metastasis formation.

Metastasized $\mathrm{PCa}$ is treated by radiation, chemotherapy and androgen deprivation therapy (ADT). First-line chemotherapy for PCa is treatment with doxetaxel or cabazitaxel (American cancer society, 2016). To lower side effects, therapy is often combined with prednisone treatment (Teply et al. 2016). For ADT, commonly used agents are bicalutamide and enzalutamide. ADT aims to inhibit androgen receptor (AR) signaling by blocking the AR ligand binding domain or inhibit AR ligand synthesis. Aberrant AR signaling is of major importance for PCa growth and its inhibition temporary discontinues tumor growth. Although treatment increases progression free survival by 2-4 years (Robert-Koch-Institut, Gesellschaft der epidemiologischen Krebsregister in Deutschland 2015), ADT is connected to serious side effects such as impotence, osteoporosis, anemia and fatigue (American cancer society, 2016) and finally, the treatment is only palliative. Almost all PCas develop adaptation mechanisms to all currently known treatment options and become castrationresistant prostate cancer (CRPC). CRPC is defined as a PCa under treatment with a total serum testosterone of under $0.5 \mathrm{ng} / \mathrm{mL}$ (or $1.7 \mathrm{nmol} / \mathrm{L}$ ) with three successive increases in PSA levels (Heidenreich et al. 2014). Different approaches were performed to efficiently target CRPC, but to date no agent was identified that suppresses tumor relapse.

\subsection{AR-targeted PCa treatment strategies and why they fail}

PCa cells are dependent on AR signaling which is activated by testosterone, respectively its metabolite dihydrotestosterone (DHT). ADT blocks this signaling and therefore inhibits tumor growth. On the one hand, the termination of AR signaling can be achieved by depletion of testosterone (castration), and on the other hand by blockade of AR signaling. Castration can be attained chemically by treatment with analogs of the luteinizing hormone-releasing hormone (LHRH), e.g. Gonadorelin or surgically by excision of testes, both terminating the production of testosterone (American cancer society, 2016). Blockade of the AR can be achieved by antiandrogens that inhibit its activation by competitive binding to the ligand binding site. Therefore, AR signaling is blocked even in the presence of DHT. These treatment options delay PCa progression and induce cell death and cell cycle arrest, but cancer cells adapt to the androgen deprivation. Different adaptation mechanisms were described, including AR amplification, gain of function mutations of the AR, AR modifications, constitutively active splice variants, gain or loss of AR cofactors or intratumoral ligand 
synthesis (Fig. 1.1) (Knudsen, Penning 2010). All these adaptations lead to a restored function of the AR and therefore result in therapeutic failure and tumor growth progression.

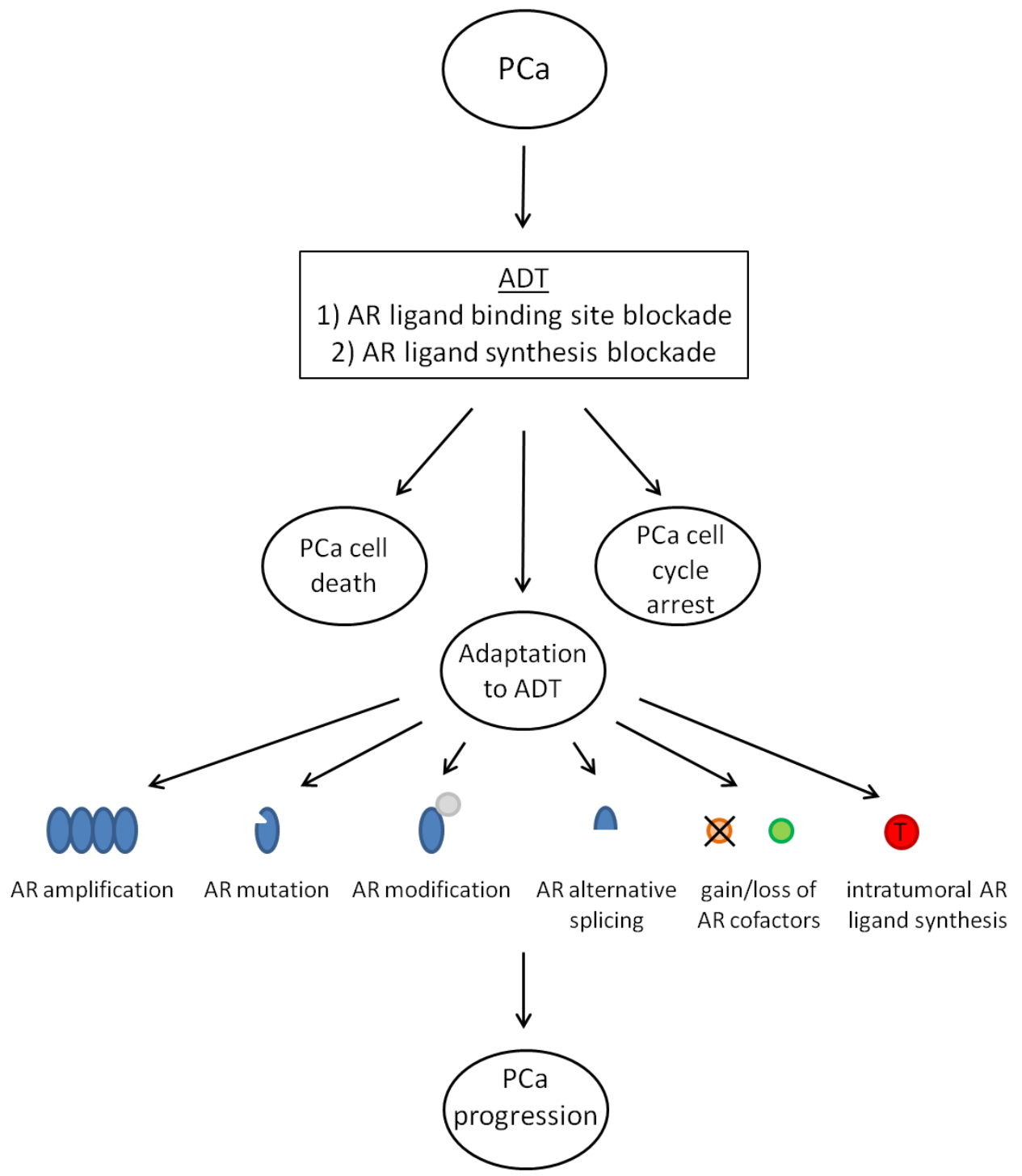

Figure 1.1: AR-mediated resistance to androgen deprivation therapy (ADT) in PCa. Androgen deprivation leads to initial tumor shrinkage as androgen signaling is the major cell growth-promoting pathway in PCa. Lately, the tumors develop resistance mechanisms leading to tumor recurrence. In this case, AR signaling is reactivated in the absence of exogenous ligands, e.g. due to AR amplification and overexpression, mutations in the ligand-binding domain of the AR, AR modifications such as phosphorylation, constitutively active AR splice variants (ARVs), differentially expressed cofactors of the AR or intratumoral AR ligand synthesis. All of these adaptations lead to a restored activity of AR signaling and therefore to PCa progression. Modified according to Knudsen, Penning (2010).

In order to cease the development of resistances two main approaches were performed to improve ADT. The first approach aims to improve the interruption of AR ligand synthesis using abiraterone acetate. Abiraterone acetate is a steroidal androgen synthesis inhibitor. It inhibits the enzyme 17a-hydroxylase (CYP17), which catalyzes the conversion of pregnenolone and progesterone to their 17a-hydroxy derivatives and subsequently the 
formation of dehydroepiandrosterone and androstenedione. Compared to its predecessors, abiraterone acetate impedes essential steps of androgen synthesis and not only the release of testosterone. Second, antiandrogens with higher affinity to the AR were developed. Enzalutamide or MDV3100 is a currently investigated second-generation synthetic nonsteroidal antiandrogen. It binds to the ligand binding site of the AR with higher specificity than its predecessors flutamide and bicalutamide (Rodriguez-Vida et al. 2015). Enzalutamide inhibits AR translocation into the nucleus and its binding to DNA (Tran et al. 2009). Furthermore, enzalutamide causes fewer side effects than its predecessors (Keating 2015). Although both approaches, i.e. indirect or direct inhibition of AR signaling, exhibit advantages over former drugs, they are finally circumvented by tumor resistance mechanisms. Even more, the adaptation to the ADT leads to cross resistances between both agents (Schrader et al. 2014; Loriot et al. 2013). The most commonly described escape mechanism to these new drugs is the expression of constitutively active AR splice variants (Antonarakis et al. 2014; Buttigliero et al. 2015; Mostaghel 2013; Mostaghel et al. 2011). Furthermore, as already observed after bicalutamide therapy, the treatment with enzalutamide results in the occurrence of $A R$ gene mutations leading to the conversion of enzalutamide from an $A R$ antagonist to an AR agonist (Korpal et al. 2013).

\subsection{New treatment strategies for prostate cancer}

First approaches to use blockade of AR signaling as a treatment for PCa were described by Charles Huggins in 1941, using castration and estrogen application for treatment of PCa (Huggins, Hodges 2002). Although drugs improved since this time, targeting the AR signaling still ends up in the development of CRPC. Therefore, different new approaches were established to treat PCa without directly targeting AR signaling.

The inhibition of PI3K (phosphatidylinositol-4,5-bisphosphate 3-kinase) signaling in CRPC is such a promising approach. PI3K is an important pathway promoting cell survival and cancer growth (Edlind, Hsieh 2014). In PCa, inhibition of PI3K inhibits cell survival very effectively, especially when combined with AR inhibition (Edlind, Hsieh 2014; Qi et al. 2015). PI3K inhibition is also supposed to decrease PCa cell invasiveness (Shukla et al. 2007) and expression of angiogenesis marker vascular endothelial growth factor (VEGF) in vitro (Fang et al. 2007). Clinical studies with PI3K inhibitors or inhibitors of PI3K targets are currently ongoing, albeit first results indicate that $\mathrm{PI} 3 \mathrm{~K}$ inhibition alone is not sufficient to treat $\mathrm{PCa}$ (Tang, Ling 2014).

Other promising approaches aim to inhibit vascularization of tumors by e.g. bevacizumab, target gene expression via histone deacetylase inhibitors, e.g. by hydroxamic acids or modifications in promoter methylation, e.g. by 5-azacytidine (Kluetz et al. 2010; Abbas, Gupta 2008; Kikuno et al. 2008; Massie et al. 2016; Graca et al. 2016). Furthermore, several 
alternative drugs (agents of natural occurrence without scientifically proven efficacy), e.g. lycopene or vitamin D are under investigation (Chen et al. 2013; Beer et al. 2003).

In this study, two new PCa treatment approaches were investigated in more detail: first, the role and efficacy of estrogen receptor (ER)-targeted therapy was analyzed, and second, the effects of the putative anti-cancer drug amygdalin were studied.

\subsubsection{ER $\beta$ as a potential target in PCa treatment}

\subsubsection{Estrogen receptors- structure and signaling}

Estrogen receptors (ER) are expressed in many tissues, e.g. lung, brain and prostate (Couse et al. 1997). The two known ER subtypes, ER $\alpha$ and ER $\beta$, were encoded by two different genes. ESR1 is located on chromosome 6q24-q27 and encodes for ER a (Gosden et al. 1986). ESR2 is located on chromosome 14q22-q24 and encodes for ER $\beta$ (Enmark et al. 1997). Both receptors show great similarities in amino acid sequence and structure. ERs have five functional domains (Fig. 1.2). The A/B domain is located at the $\mathrm{N}$-terminus and is responsible for transcriptional activity and interaction with co-regulators (activation function 1 or AF-1). Here, ER $\alpha$ and ER $\beta$ show strong differences with only $30 \%$ conserved regions. The $\mathrm{C}$ domain encodes the DNA binding domain (DBD) (96\% homology) (Sotoca et al. 2012). The $D$ domain encodes the nuclear localization signal (NLS) domain and ER $\alpha$ and ER $\beta$ share $30 \%$ homology in this region (Sotoca et al. 2012). The $E$ domain is located on the C-terminal region and encodes the ligand binding domain (LBD) and the F domain encodes for the AF-2 (activation function 2), which is involved in regulation of transcriptional activity and interaction with various co-regulators (Edwards 2000). ER $\alpha$ and ER $\beta$ share a sequence identity of $53 \%$ in the LBD. Presumably, the differences in the F domains of the ERs contribute to the ability of ERs to selectively control transcription of target genes (Skafar, Zhao 2008; Lee et al. 2012; Kong et al. 2003).

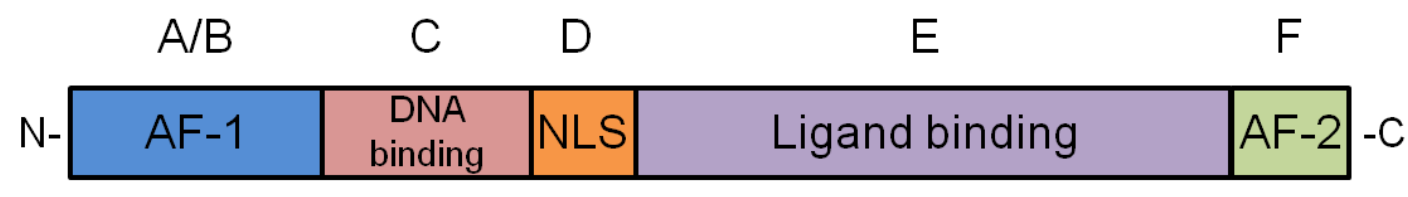

\begin{tabular}{|c|c|c|c|}
\hline $\begin{array}{l}\text { Homology } \\
F R \alpha / F R B\end{array}$ & $30 \%$ & $96 \%$ & $30 \%$ \\
\hline
\end{tabular}

Figure 1.2: Schematic illustration of ER domain structure. ERs show five functional domains. The $A / B$ domain encodes for the activation function $1(A F-1)$, determining transcriptional activity and interaction with cofactors. The $\mathrm{C}$ domain encodes for the DNA binding domain. Domain $\mathrm{D}$ contains the nuclear localization signal (NLS). The $E$ domain determines the ligand binding domain and domain $F$ encodes for the AF-2. Modified according to Huang et al. (2010) and Sotoca et al. (2012).

ERs belong to the steroid receptor family. Other steroid receptors are the androgen receptor, the progesterone receptor, the glucocorticoid receptor and the mineralocorticoid receptor. 
Steroid receptors are involved in the regulation of gene expression. They either function as transcription factors (ligand-dependent and ligand-independent) or mediate nontranscriptional effects via second messenger activation (Fig. 1.3) (Cui et al. 2013; Lonergan, Tindall 2011; Liao et al. 2013). One of the best described molecular mechanisms is the function of steroid receptors as ligand-dependent transcription factors. Ligand binding initiates dimerization of steroid receptors and translocation into the nucleus. The endogenous ligand for both ER subtypes is the steroid hormone estrogen (E2). Each steroid receptor regulates the expression of a specific set of genes. Steroid receptors can form heterodimers with other members of their class or different splice variants and thereby change transcriptional activity and target gene sets (Cowley et al. 1997; Li et al. 2004). Receptor activation can also be mediated via phosphorylation, e.g. due to the activation of receptor tyrosine kinases and their downstream targets PI3K or MAPK. Furthermore, steroid receptors can be membrane-bound and upon ligand binding, the steroid receptors activate second messengers, e.g. G protein-coupled receptors or calcium release. These second messengers either activate transcription factors and therefore regulate gene transcription or activate non-genomic processes (Cui et al. 2013). 


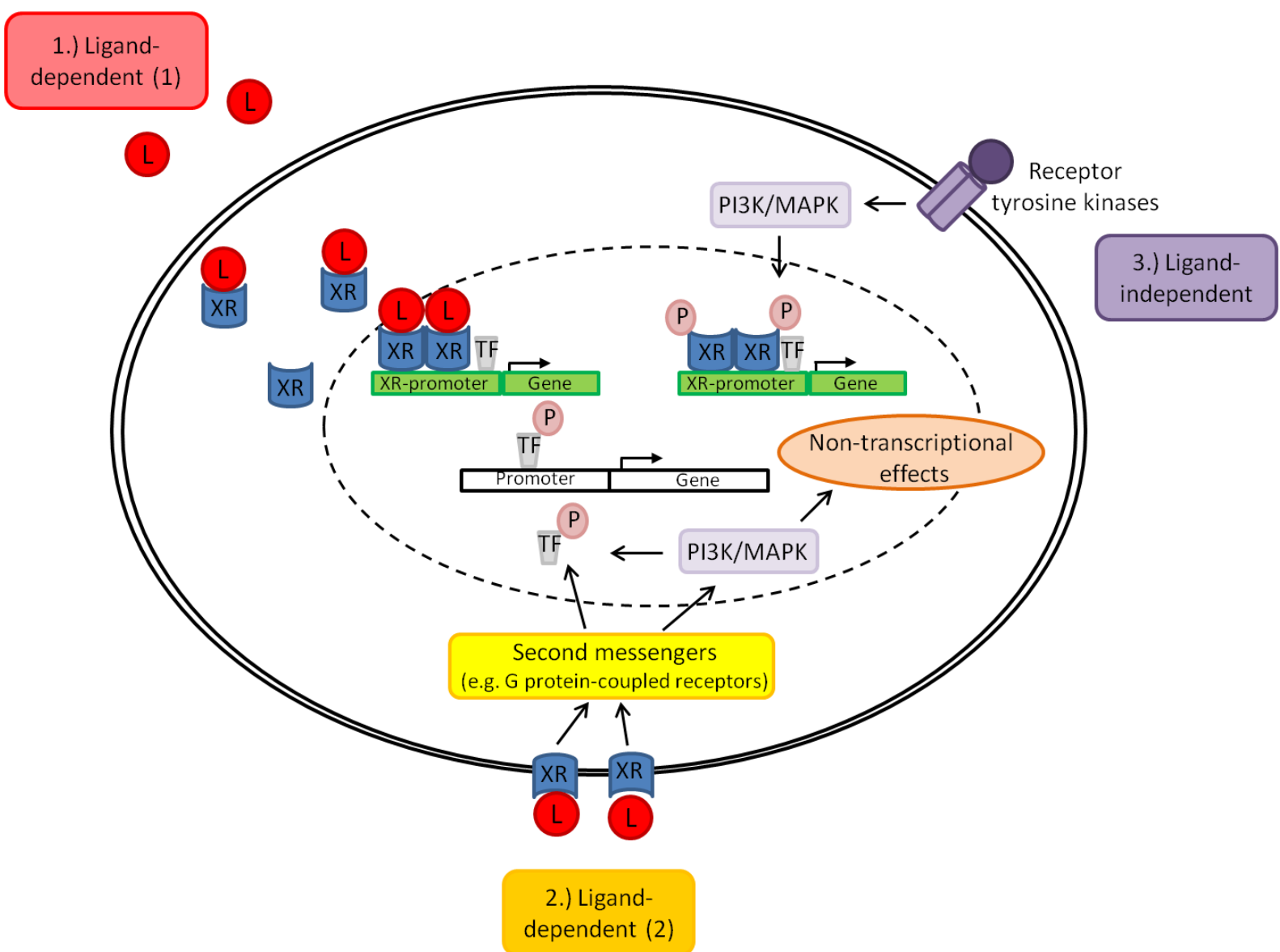

Figure 1.3: Signaling of steroid receptors. Steroid receptors $(X R)$ are involved in gene expression. They can act either as transcription factors (TF) or regulate transcription of several genes. The signaling of steroid receptors can be activated by different pathways. The best described activation of the steroid receptors is mediated by dimerisation and translocation into the nucleus upon ligand $(\mathrm{L})$ binding (1. ligand-dependent (1)). Here, the ligand-bound steroid receptors directly bind to their target DNA and initiate transcription. Steroid receptors can also be membrane-bound. Upon ligand binding, second messengers (e.g. G protein-coupled receptors) are activated. These messengers either activate transcription factors and therefore regulate gene transcription or activate non-genomic processes (2. ligand-dependent (2)). Steroid receptor activation can also be mediated via phosphorylation, e.g. due to the activation of receptor tyrosine kinases and their downstream targets PI3K or MAPK (3. ligand-independent). Modified according to Cui et al. (2013), Lonergan, Tindall (2011) and Liao et al. (2013).

\subsubsection{Estrogens and their receptors in PCa}

Estrogens have a long history in PCa treatment. In 1941, estrogens were found to induce chemical castration in men with metastasized PCa. Estrogen treatment downregulates testosterone synthesis by feedback inhibition of hypothalamic and pituitary luteinizing hormone release. However, treatment with estrogens caused severe side effects such as cardiac failure and thrombosis (Klotz et al. 1999). Therefore, estrogen treatment was replaced by treatment with $\mathrm{LHRH}$ analogs. More recently, phytoestrogens came up as an alternative treatment for PCa. Phytoestrogens naturally occur in soy, whole grain and some 
seeds. They show structural similarities to estrogens and there are three classes of phytoestrogens: lignans, isoflavones and coumestans. The initial assumption that phytoestrogens affect $\mathrm{PCa}$ is based on the hypothesis that the low $\mathrm{PCa}$ risk in the Asian population is associated to the phytoestrogen-rich diet. Different studies and approaches were made to elucidate the effect of phytoestrogens on PCa and research is still ongoing. In summary, the greatest therapeutically significance is attained by isoflavones, e.g. tectorigenin and genistein. Isoflavones cause cell growth arrest and apoptosis in PCa, which was shown both in vitro and in vivo (Kumi-Diaka et al. 2000; Mentor-Marcel et al. 2001; Wang et al. 2007; Shen et al. 2000). However, the underlying mechanism of action of isoflavones in PCa remains largely unknown. Antiandrogenic function, antioxidative features, inhibition of 5alpha-reductase, 17beta-hydroxysteroid dehydrogenase, aromatase, tyrosinespecific protein kinases and DNA topoisomerase II are described to be involved in phytoestrogen-mediated effects (Morrissey, Watson 2003, 2003; Goetzl et al. 2007). Due to their structural similarity to estrogen, it is supposed that phytoestrogens activate estrogen receptors and thus induce treatment effects. Further analyses revealed that phytoestrogens showed greater affinity to the estrogen receptor subtype $\beta$ (ER $\beta$ ) than to the estrogen receptor subtype $\alpha$ (ER $\alpha$ ) (Turner et al. 2007). The differences between ER $\alpha$ and ER $\beta$ were taken into account and advantages of ER subtype-specific activation became a new subject of investigation.

Although the ER subtypes show strong sequence homologies and are activated by the same ligand, they are supposed to exhibit antagonistic functions (Lindberg et al. 2003; Prins, Korach 2008). ER $\alpha$ is known to promote cell growth and proliferation, whereas ER $\beta$ inhibits proliferation and is under investigation as a potential tumor suppressor in different cancer types, such as breast cancer and prostate cancer (Palmieri et al. 2002; Lazennec 2006). Moreover, in studies performed by Slusarz et al. (2012) knockout mouse models with either ER $\alpha$ knockout (ERKO) or ER $\beta$ knockout (BERKO) were used. To generate mouse models for investigation of ERs in PCa, ERKO and BERKO mice were crossed to TRAMP mice. TRAMP (transgenic adenocarcinoma of mouse prostate) mice express the simian virus (SV) $40 \mathrm{~T} / \mathrm{t}$ antigen under the control of the prostate-specific probasin promoter. SV $40 \mathrm{~T} / \mathrm{t}$ is an oncogene, inducing the malignant degeneration of cells. The TRAMP mouse model was extensively studied and is an established model for PCa (Hurwitz et al. 2001). Slusarz et al. (2012) demonstrated that ERKO/TRAMP mice developed less aggressive PCa, while BERKO/TRAMP mice develop PIN lesions at an earlier time point with more aggressive course as observed in TRAMP mice. Furthermore, the role of ER $\beta$ as a tumor suppressor in PCa emerged as studies showed that ER $\beta$ expression decreases during PCa progression (Stettner et al. 2007) and that the re-expression of ER $\beta$ in PCa inhibits tumor cell growth in vitro (Dey et al. 2012; Dey et al. 2014). The activation of ER $\beta$ by ER $\beta$ agonist treatment in PCa cell lines induced apoptosis (Dey et al. 2014), induced cell cycle arrest (Hurtado et al. 
2008; Nakamura et al. 2013) and reduced cell invasiveness (Cheng et al. 2004). However, the effect of ER $\beta$ in PCa is still controversially discussed. For example, Leung et al. (2010) described that ER $\beta$ splice variant 2 and 5 exhibit tumor promoting effects. In addition, Horvath et al. (2001) described a theory of switching roles for ER $\beta$ in which ER $\beta$ signaling changed from tumor-suppressive in early $\mathrm{PCa}$ stages to tumor-promoting signaling in advanced PCa. The modification of function is supposed to be mediated via interactions with other nuclear receptors, e.g. the AR (Zellweger et al. 2013) or increased expression of ER $\beta$ splice variant 2 (Dey et al. 2012). To date, the molecular processes of ER action in PCa are largely unknown and need to be further elucidated.

\subsubsection{Amygdalin as a potential drug in PCa treatment}

\subsubsection{The active agent amygdalin}

Amygdalin is a natural occurring cyanogenic diglucoside $\left(\mathrm{C}_{20} \mathrm{H}_{27} \mathrm{NO}_{11}\right)$ found in seeds of apricots, peaches, cherries and bitter almonds (Holzbecher et al. 1984; Bolarinwa et al. 2014). In 1837, amygdalin was separated and purified by the chemists Robiquet and Boutron (Dorr, Paxinos 1978). It was first described in 1845 as a drug for cancer treatment. In the following years, different derivates of amygdalin occurred and were used for treatment of various cancer entities and other diseases, e.g. for antitussive and anti-asthmatic uses, antiatherogenic use, treatment of renal interstitial fibrosis, prevention of pulmonary fibrosis, treatment of hyperoxia induced lung injury, immune suppression, immune regulation, antiinflammatory use and antiulcer use (Song, Xu 2014). Ernst T. Krebs, Sr. and his son Ernst T. Krebs, Jr. claimed amygdalin to be an effective anti-cancer drug in the 1940s and 1950s (Unproven methods of cancer management. Laetrile 1991). But evidence for this statement was missing. Different studies by the American food and drug administration (FDA) could not confirm amygdalins' effectiveness in cancer treatment but emphasized the toxic properties. Therefore, amygdalin was banned from the market in the USA and Europe in 1987 (Song, Xu 2014). Despite that, amygdalin remained a popular alternative drug in Mexico. In the past decade, amygdalin came back into the focus of scientists, and studies concerning amygdalins' efficacy were resumed. So far, the analgesic effect of amygdalin could be shown in reliable in vivo studies (Hwang et al. 2008). Evidence was found that amygdalin also exhibits anti-tussive and anti-asthmatic features (Do et al. 2006; Chang et al. 2005).

Amygdalin is used as an alternative treatment of $\mathrm{PCa}$, but without reliable studies concerning its efficacy. In contrast, early clinical studies by Moertel et al. (1982) found no evidence for anti-cancerous effects of amygdalin. However, this clinical trial lacked proper study design. Recent publications support anti-cancerous effects of amygdalin. In PCa cell culture, amygdalin treatment induced apoptosis (Chang et al. 2006) and $\mathrm{G}_{2}$-phase arrest (Makarevic et al. 2016). Furthermore, apoptosis-inducing and cell cycle-regulating effects of amygdalin were observed in a number of cancer types in vitro such as leukemia, colon cancer and 
prostate cancer (Song, Xu 2014). To date, the molecular mechanism of amygdalin action is unknown and up to now reliable in vivo analyses are not available.

\subsubsection{Mechanism of metabolism of amygdalin}

Amygdalin itself is a non-toxic substance and it must be metabolized to release potentially anti-cancerous agents. Haisman and Knight (1967) showed that amygdalin can be cleaved in a hydrolytic enzymatic reaction by $\beta$-glucosidase. Hereby, amygdalin is disassembled into its components glucose, benzaldehyde and cyanide. The cyanide ion has cell toxic properties and therefore is assumed to be responsible for the anti-cancerous effects. The benzaldehyde molecule has pain-relieving properties. Additional, enzymatic reactions catalyzed by the enzyme rhodanase detoxicate released cyanide ions (see Fig. 1.4). Indeed, it remains unclear if the reaction shown by Haisman and Knight (1967) truly expresses how amygdalin is metabolized in vivo, since reliable studies are missing.
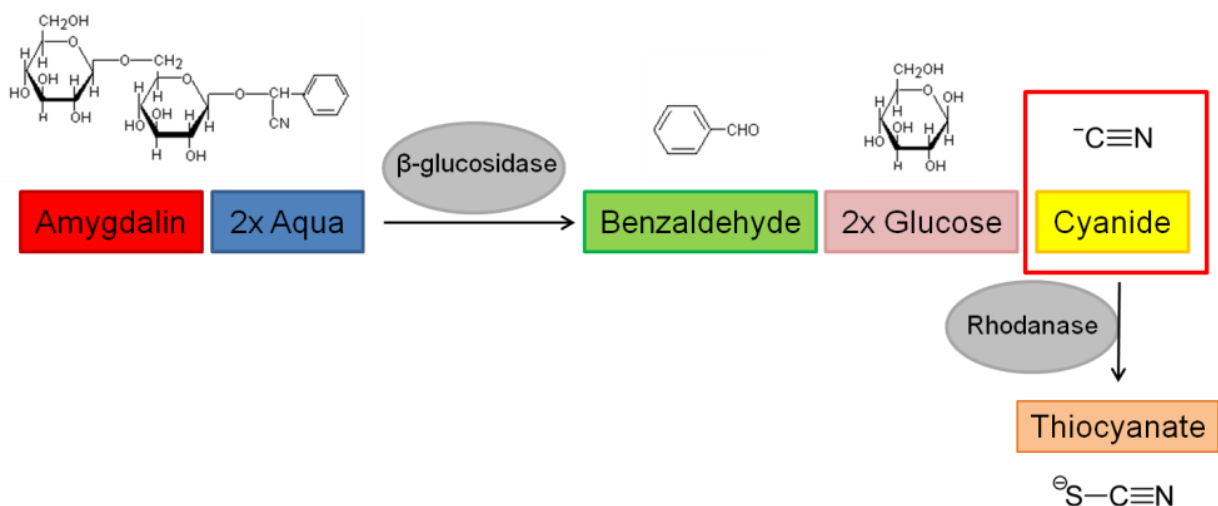

Figure 1.4: Hypothesized mechanism of amygdalin metabolism. To date, it is still unclear, how amygdalin is metabolized in vivo. One possible mechanism is the cleavage of amygdalin by the enzyme $\beta$-glucosidase. Products of this reaction are benzaldehyde, glucose and cyanide. Cyanide has cell toxic properties. The enzyme rhodanase could further detoxicate cyanide into thiocyanate by adding a sulfide group. Modified according to Balkon (1982).

\subsection{Aims of the study}

This study aims to elucidate the effectiveness of two possible PCa treatments. First, the role of estrogen receptors, especially the ER $\beta$, in PCa was examined. The effects of ER $\beta$ activation on PCa cells and the underlying molecular signaling were studied. Second, the effect of amygdalin on PCa development and molecular signaling was analyzed in vivo and in vitro.

The main aims were:

- Analysis of the influence of ER $\alpha$ or ER $\beta$ knockout on PCa development and progression in the TRAMP mouse model

- Histopathological analysis of tumor development, incidence and stage 
- Comparison of survival time of TRAMP, ERKO/TRAMP and BERKO/TRAMP mice

- Generation of ER $\alpha$ or ER $\beta$ knockout murine, primary PCa cell lines

- Analysis of primary PCa cell proliferation rate

- Analysis of primary PCa cell signaling

- Analysis of the invasive potential of primary PCa cells

- Confirmation of the VCaP cell system (VCaP rev., VCaP and VCaP AA) as a model resembling different human PCa stages

- Analysis of differential expression of PCa relevant genes in VCaP cell variants

- Analysis of the effect of ER $\beta$ activation in VCaP cell variants

- Analysis of proliferation rate and apoptosis induction

- Analysis of expression of PCa relevant genes

- Identification of signaling mechanisms in VCaP cell variants

- Whole mRNA-sequencing analysis of differentially expressed genes

- Identification of candidate genes and pathways

- Analysis of the ER pathway

- Analysis of the cholesterol synthesis pathway

- Analysis of the PI3K pathway

- Analysis of AR-mediated signaling

- Analysis of the effect of amygdalin on TRAMP-FVB mice

- Analysis of side effect occurrence by observation of behavior and weight

- Histopathological analysis of toxic side effects

- Monitoring of PCa development, progression and stage

- Analysis of mechanism of metabolisation

- Identification of cell signaling

- In vitro studies of the effect of amygdalin on primary, murine PCa cell lines T244 and $2 \mathrm{E}$

- Analysis of cell survival, cytotoxicity and apoptosis induction

- Analysis of invasiveness

- Expression analysis of proliferation marker Ki67

- Analysis of induction of senescence by senescence-associated $\beta$ galactosidase staining

- Analysis of senescence inducing factors 
- $y \mathrm{H} 2 \mathrm{Ax}$ expression as indication for DNA damage

- Damaged nuclei as indicators for mitotic catastrophe 


\section{Material and Methods}

\subsection{Chemicals and Reagents}

Table 2.1: Chemicals used in this study.

\begin{tabular}{|c|c|}
\hline Chemical & Company \\
\hline $8 \beta-V E 2$ & Bayer AG, Leverkusen \\
\hline $\begin{array}{l}\text { 5-bromo-4-chloro-3-indolyl } \alpha \text {-D- } \\
\text { galactopyranoside (X- } \alpha \text {-Gal) }\end{array}$ & Biomol, Hamburg \\
\hline Abiraterone acetate & Janssen Cilag, Neuss \\
\hline Agar & Carl Roth GmbH, Karlsruhe \\
\hline Agarose & Thermo Scientific, Langenselbold \\
\hline Aminoglutethimide & Sigma-Aldrich, Taufkirchen \\
\hline Amygdalin & Sigma-Aldrich, Taufkirchen \\
\hline Amygdalin (purified) & CuraFaktur GmbH, Heilbronn \\
\hline Ampicillin & Sigma-Aldrich, Taufkirchen \\
\hline Ampuwa & Fresenius AG, Bad Homburg \\
\hline Chloramine-T & Sigma-Aldrich, Taufkirchen \\
\hline Chloroform & Carl Roth GmbH, Karlsruhe \\
\hline Dextran-coated charcoal & Sigma-Aldrich, Taufkirchen \\
\hline DHT & Sigma-Aldrich, Taufkirchen \\
\hline Dichlormethan & Promochem, Wesel \\
\hline Dipotassium phosphate & AppliChem GmbH, Darmstadt \\
\hline Direct PCR Tail & Peqlab, Erlangen \\
\hline DMF & Carl Roth GmbH, Karlsruhe \\
\hline DMSO & Carl Roth GmbH, Karlsruhe \\
\hline DNA Stain G & Serva GmbH, Heidelberg \\
\hline dNTPs (100 mM) & Thermo Scientific, Langenselbold \\
\hline DPBS & PAN, Aidenbach \\
\hline DTT & Biomol, Hamburg \\
\hline EDTA & ICN Biomedicals, Aurora, USA \\
\hline ERB 041 & Tocris, Wiesbaden \\
\hline Ethanol & Chemie Vertrieb Hannover, Hannover \\
\hline FERb 033 & Tocris, Wiesbaden \\
\hline Formaldehyde & Carl Roth GmbH, Karlsruhe \\
\hline Fulvestrant & Sigma-Aldrich, Taufkirchen \\
\hline Gentamycin sulphate solution & Carl Roth GmbH, Karlsruhe \\
\hline Glacial acetic acid & Sigma-Aldrich, Taufkirchen \\
\hline
\end{tabular}




\begin{tabular}{|c|c|}
\hline Glycergel & Dako, Glostrup, Denmark \\
\hline Glycerol & Carl Roth $\mathrm{GmbH}$, Karlsruhe \\
\hline Glycine & Carl Roth $\mathrm{GmbH}$, Karlsruhe \\
\hline HBSS & Gibco, Langenselbold \\
\hline $\mathrm{HCL}$ & Carl Roth $\mathrm{GmbH}$, Karlsruhe \\
\hline Hemalum & Carl Roth $\mathrm{GmbH}$, Karlsruhe \\
\hline Hexadecyltrimethylammonium bromide & Sigma-Aldrich, Taufkirchen \\
\hline Hydrogen peroxide & Carl Roth GmbH, Karlsruhe \\
\hline Isopropanol & Carl Roth $\mathrm{GmbH}$, Karlsruhe \\
\hline Low-fat dry milk & Carl Roth GmbH, Karlsruhe \\
\hline Magnesium chloride & Bioline, Luckenwalde \\
\hline Methanol & Carl Roth GmbH, Karlsruhe \\
\hline MycoZap ${ }^{\text {TM }}$ Spray & Lonza, Cologne \\
\hline NuPAGE TM LDS Sample buffer (4x) & Thermo Scientific, Langenselbold \\
\hline NuPAGE ${ }^{\mathrm{TM}}$ MES Running buffer (20x) & Thermo Scientific, Langenselbold \\
\hline NuPAGE ${ }^{\mathrm{TM}}$ Sea Blue Plus2 & Thermo Scientific, Langenselbold \\
\hline Orange G & Sigma-Aldrich, Taufkirchen \\
\hline Penicillin/Streptomycin & PAN, Aidenbach \\
\hline Penton & Carl Roth GmbH, Karlsruhe \\
\hline $\mathrm{PFBBr}$ & Sigma-Aldrich, Taufkirchen \\
\hline Phosphatase-Inhibitor-Mix II & Serva GmbH, Heidelberg \\
\hline Phosphoric acid & Sigma-Aldrich, Taufkirchen \\
\hline PMSF & Carl Roth GmbH, Karlsruhe \\
\hline Potassium acetate & Sigma-Aldrich, Taufkirchen \\
\hline Potasium ferrocyanide & Carl Roth $\mathrm{GmbH}$, Karlsruhe \\
\hline Potasium ferricyanide & Carl Roth $\mathrm{GmbH}$, Karlsruhe \\
\hline Protease Inhibitor cocktail & Roche, Basel, Switzerland \\
\hline RNaseZAP® & Sigma-Aldrich, Taufkirchen \\
\hline Roti $^{\circledR}$-Nanoquant & Carl Roth GmbH, Karlsruhe \\
\hline Sodium hydroxid & Carl Roth $\mathrm{GmbH}$, Karlsruhe \\
\hline Testosterone & Sigma-Aldrich, Taufkirchen \\
\hline Toluol & Promochem, Wesel \\
\hline Tris & Carl Roth $\mathrm{GmbH}$, Karlsruhe \\
\hline Triton $\mathrm{X}-100$ & Fluka, Deisenhofen \\
\hline TRIzolß & Thermo Scientific, Langenselbold \\
\hline Trypsin/EDTA & PAN, Aidenbach \\
\hline Tween 20 & Merck, Darmstadt \\
\hline Vectashield with DAPI & VectorLab, Burlingame, USA \\
\hline
\end{tabular}


Xylene

Yeast extract
Carl Roth $\mathrm{GmbH}$, Karlsruhe

Carl Roth $\mathrm{GmbH}$, Karlsruhe

\subsection{Biochemicals and Enzymes}

Table 2.2: Biochemicals and enzymes used in this study.

\begin{tabular}{l|l}
\hline Biochemical & Company \\
\hline bFGF & Stemcell technologies, Cologne \\
Bovine serum albumin (BSA) & Carl Roth GmbH, Karlsruhe \\
Collagenase & Sigma-Aldrich, Taufkirchen \\
EGF & Stemcell technologies, Cologne \\
Fetal bovine serum (FBS) & PAN, Aidenbach \\
Fibronectin & Roche, Basel, Switzerland \\
Heparin & Stemcell technologies, Cologne \\
Lipofectamine® 2000 & Thermo Scientific, Langenselbold \\
Mango Taq DNA polymerase & Bioline, Luckenwalde \\
Matrigel B Basement Membrane Matrix & Corning Inc., New York, USA \\
NuSerum ${ }^{\text {TM }}$ & Corning Life Sciences, Bedford, USA \\
Phalloidin, TRITC-markiert & Sigma-Aldrich, Taufkirchen \\
Proteinase K & Thermo Scientific, Langenselbold \\
RNase A & AppliChem GmbH, Darmstadt \\
SuperScript II Reverse Transcriptase & Thermo Scientific, Langenselbold \\
VCaP Cell Avalanche® & EZ Biosystems, Washington, DC, USA \\
X-treme GENE ${ }^{\text {TM }}$ HP DNA & Roche, Basel, Switzerland \\
\end{tabular}

\subsection{Consumer goods}

Table 2.3: Consumer goods used in this study.

\begin{tabular}{|c|c|}
\hline Consumer goods & Company \\
\hline $\begin{array}{l}4 \text { chamber polystyrene vessel tissue } \\
\text { culture treated glass slide }\end{array}$ & BD Biosciences, Heidelberg \\
\hline 6-well plate & Sarstedt, Nürnbrecht \\
\hline 12-well plate & Corning Inc., New York, USA \\
\hline 24-well plate & Greiner Bio-One, Frickenhausen \\
\hline 96-well plate, translucent & Thermo Scientific, Langenselbold \\
\hline 96-well microplate, black, clear-bottom & BD Biosciences, Heidelberg \\
\hline 384-well plates, black & 4titude, Surrey, UK \\
\hline $\mathrm{BD} \quad$ BioCoat $^{\mathrm{TM}} \quad$ Matrigel $^{\mathrm{TM}}$ Invasion & BD Biosciences, Heidelberg \\
\hline Chambers & \\
\hline
\end{tabular}


Blotting paper

Cell strainer

Combitips plus, $10 \mathrm{ml}$

Cover slips, 24 × $60 \mathrm{~mm}$

Cryo.S ${ }^{\mathrm{TM}}$ cups with screw cap

Gloves

Microscope slides Superfrost Plus

Neubauer improved counting chamber

Nunclon ${ }^{\mathrm{TM}}$ surface 96-well plate

NuPAGE ${ }^{\text {TM }} 4-12 \%$ Bis-Tris gels

Pasteur pipettes

Pipet tips

PVDF transfer membrane

Reaction tubes, $0.2 \mathrm{ml}$

Reaction tubes, $1.5 \mathrm{ml}$

Reaction tubes, $2 \mathrm{ml}$

Round bottom tube

Serological pipettes $(5 \mathrm{ml}, 10 \mathrm{ml})$

Stainless steel bead

Sterican Needle, $\varnothing 0.45 \times 12 \mathrm{~mm}$

Sterile filter

Sterile surgical blades

Syringe

Tissue culture flask, $25 \mathrm{~cm}^{2}$

Tissue culture flask, $75 \mathrm{~cm}^{2}$

Tube, $13 \mathrm{ml}$

Tube, $15 \mathrm{ml}$

Tube, $50 \mathrm{ml}$

qPCR Adhesive Clear Seals
Schleicher \& Schüll, Dassel

BD Biosciences, Heidelberg

Eppendorf, Hamburg

Menzel Gläser, Braunschweig

Greiner Bio-One, Frickenhausen

Sempermed, Neuwied

Schütt, Göttingen

Hartenstein, Würzburg

Thermo Scientific, Langenselbold

Thermo Scientific, Langenselbold

Brand, Wertheim

Sarstedt, Nürnbrecht

GE Healthcare, Munich

Sarstedt, Nürnbrecht

Sarstedt, Nürnbrecht

Sarstedt, Nürnbrecht

BD Biosciences, Heidelberg

Sarstedt, Nürnbrecht

Quiagen, Hilden

B. Braun, Melsungen

Sartorius, Göttingen

Braun, Tuttlingen

BD Biosciences, Heidelberg

Sarstedt, Nürnbrecht

Sarstedt, Nürnbrecht

Sarstedt, Nürnbrecht

Greiner Bio-One, Frickenhausen

Greiner Bio-One, Frickenhausen

4titude, Surrey, UK

\subsection{Instruments}

Table 2.4: Instruments used in this study.

\begin{tabular}{l|l}
\hline Instrument & Company \\
\hline ABI 3500XL genetic analyzer & Applied biosystems, Darmstadt \\
Accu-jet® (Pipet-Boy) & Brand, Wertheim \\
Confocal Laser Scanning Microscope & Olympus GmbH, Hamburg \\
IX81 &
\end{tabular}


Centrifuge Heraeus Fresco 21

Centrifuge Heraeus Pico 21

Centrifuge 4K15

Circular rotor

$\mathrm{CO}_{2}$ Incubator MCO-20AIC

Confocal laser scanning microscope IX81

ECX-F20.M transilluminators

Electro-blotter

Electrophoresis power supply PS 304

FluorChem ${ }^{\circledR} \mathrm{Q}$

Fluorescence microscope BX60

Gasprofi 1SCS micro

GC-MS QP2010plus

Headspace-Sampler Turbomatrix

HiSeq 2000

Hood SterilGARD

Incubator Heraeus

Inverted microscope IX71

Magnetic stirrer MR Hei-standard

MegaBace $^{\text {TM }} 1000$

Multipette ${ }^{\circledR}$ plus

$\mathrm{pH}$ meter

Pipettes

Primus 25advanced Thermocycler

Roll mixing device RM 5

Scale Quintix

Shaker RSU-310 R

Sonication device, Bioruptor plus, UCD300

Sterioscope Olympus SZX12

Synergy $M x$

Thermocycler Primus 25 advanced

Thermomixer compact

Tissue embedding cassettes

TissueLyser LT

TRACE ${ }^{\text {TM }}$ Ultra Gas Chromatograph

Transferpette ${ }^{\circledR}-8$
Thermo Scientific, Langenselbold

Thermo Scientific, Langenselbold

Sigma-Aldrich, Taufkirchen

Sprout, Vernon Hills, USA

Sanyo, München

Olympus, Hamburg

Vilber, Eberhardzell

Biometra, Göttingen

Thermo Scientific, Langenselbold

Alpha Innotech, Logan, Utah, USA

Olympus GmbH, Hamburg

WLD-Tec, Göttingen

Shimadzu Scientific Instruments, Columbia, USA

PerkinElmer, Waltham, USA

Illumina San Diego, USA

The Baker Company, Sanford, USA

Thermo Scientific, Langenselbold

Olympus GmbH, Hamburg

Heidolph, Schwabach

GE Healthcare, Freiburg

Eppendorf, Hamburg

Mettler Toledo, Gießen

Gilson, Middleton, USA

Peqlab, Erlangen

Karl Hecht GmbH\&Co KG, Sondheim

Sartorius, Göttingen

Bolatec, Gladenbach

Diagenode, Liège, Belgium

Olympus $\mathrm{GmbH}$, Hamburg

Bio Tek, Winooski, USA

Peqlab, Erlangen

Eppendorf, Hamburg

NeoLab, Heidelberg

Quiagen, Hilden

ThermoFisher Scientific, Dreieich

Brand, Wertheim 
Vacuum centrifuge

Vortex-Genie 2

Water bath
Thermo Scientific, Langenselbold

Scientific Industries, Karlsruhe

GFL, Burgwedel

\subsection{Reaction systems}

Table 2.5: Reaction systems used in this study.

\begin{tabular}{l|l}
\hline Reaction system & Company \\
\hline ApoTox-Glo ${ }^{\text {TM }}$ Triplex Assay & Promega, Fitchburg, USA \\
CellTiter 96® AQuaous Non-Radioactive & Promega, Fitchburg, USA \\
Proliferation Assay (MTS) & \\
Diff-Quick Staining Set & Dade Behring GmbH, Marburg \\
Dual-Luciferase® Reporter Assay System & Promega, Fitchburg, USA \\
ECL Prime $\quad$ Systems & Ge Healthcare, Freiburg \\
EnVision Glostrup, Denmark \\
Peroxidase/DAB, Rabbit/Mouse & \\
ImmunoPen & Millipore, Billerica, USA \\
Myco Alert® Mycoplasma Detection Kit & Lonza, Cologne \\
Mycokill AB & Lonza, Cologne \\
peqGold Total RNA Kit & PeqLab, Erlangen \\
PhosSTOP Phosphatase Inhibitor & Roche, Mannheim \\
PureLinkTM HiPure Plasmid Midiprep Kit & Thermo Scientific, Langenselbold \\
Smarter Nucleic Acid Sample Preparation & Stratec, Berlin \\
TruSeq RNA Library Preparation Kit v2 & Illumina, San Diego, USA
\end{tabular}

\subsection{Buffers and Solutions}

Table 2.6: Buffers and solutions. Distilled and filtered water was used to set up buffers and solutions.

\begin{tabular}{l|l}
\hline Buffer/solution & Composition \\
\hline Acetate buffer, 1 M, pH 5.5 & $\begin{array}{l}5.758 \mathrm{ml} \text { glacial acetic acid } \\
100 \mathrm{ml} \mathrm{H}_{2} \mathrm{O} \\
\text { adjust to } \mathrm{pH} 5.5 \text { with } \mathrm{NaOH}\end{array}$ \\
Blocking buffer (immunofluorescence) & $3 \% \mathrm{BSA}$ \\
$1 \times$ DPBS & \\
Blocking buffer (IHC) & $3 \%$ BSA \\
& $5 \%$ FBS \\
& $1 \times$ DPBS
\end{tabular}


Blocking buffer (WB)

Citric buffer

Derivatisation solution

Fixing solution

Loading buffer (Gel electrophoresis)

Modified RIPA buffer

$\mathrm{NaCl}$ solution

Staining solution (senescence staining)
$1 \times$ TBS/ $0.1 \%$ Tween 20

$5 \%$ low-fat dry milk

$10 \mathrm{mM}$ Citrate

Adjust to $\mathrm{pH} 6$ with $\mathrm{NaOH}$

300 mg Hexadecyltrimethylammonium bromide $10 \mathrm{ml}$ Dichlormethan

$3 \mathrm{ml}$ Methanol

$130 \mu \mathrm{PFBBr}$

$3.7 \%$ Formaldehyde

$1 \times$ DPBS

$0.15 \mathrm{~g} / \mathrm{ml}$ Ficoll 400

$12.7 \mathrm{mM}$ EDTA

$5.53 \mathrm{mM}$ Orange $\mathrm{G}$

$1 \%$ Gylcerine

$50 \mathrm{mM}$ Tris $\mathrm{pH} 7.4$

$1 \%$ NP-40

$0.25 \%$ Na-deoxycholate

$150 \mathrm{mM} \mathrm{NaCl}$

1 mM EDTA

$10 \mu \mathrm{l} / \mathrm{ml}$ Protease Inhibitor cocktail

PhosphoStop Phosphatase Inhibitor (1 tablet in $10 \mathrm{ml}$ buffer)

$1 \mathrm{mM}$ PMSF

$50 \mathrm{~g} \mathrm{NaCl}$

$100 \mathrm{ml} \mathrm{H}_{2} \mathrm{O}$

$100 \mathrm{mM}$ potasium ferrocyanide

$100 \mathrm{mM}$ potasium ferricyanide

$1 \mathrm{M} \mathrm{MgCl}_{2}$

$1 \mathrm{mg} / \mathrm{ml} \mathrm{Xgal} \mathrm{in} \mathrm{DMF}$

adjust to $\mathrm{pH} 6.0$ with $\mathrm{HCL}$ 


\begin{tabular}{l|l} 
10x TBS & $\begin{array}{l}1.37 \mathrm{M} \mathrm{NaCl} \\
100 \mathrm{mM} \text { Tris / HCl, pH 7.6 } \\
\text { Transfer buffer Ila (WB) } \\
25 \mathrm{mM} \text { Tris, pH } 8.3 \\
192 \mathrm{mM} \text { Glycine } \\
20 \% \text { Methanol }\end{array}$ \\
20x Turbo buffer & $\begin{array}{l}0.2 \mathrm{M} \mathrm{NaOH} \\
\text { adjust with solid } \mathrm{H}_{3} \mathrm{BO}_{3} \text { to } \mathrm{pH} 8.0 \\
1 \times \mathrm{TBS}-\mathrm{T} \\
\text { Washing solution (WB) }\end{array}$ \\
X-Gal-Stock solution & $\begin{array}{l}2.5 \% \text { low-fat dry milk } \\
100 \mathrm{mg} / \mathrm{ml} \text { X-Gal in DMF }\end{array}$
\end{tabular}

\subsection{Culture media and Agar plates}

\subsubsection{Culture media for eukaryotic cell cultures}

Media used for the cultivation of eukaryotic cell lines were purchased from PAN (Aidenbach), Gibco (Langenselbold) or Stemcell technologies (Cologne). Additionally, fetal bovine serum (FBS) (PAN, Aidenbach) and antibiotics (PAN, Aidenbach and Carl Roth GmbH, Karlsruhe) were added. Following media were used for eukaryotic cell culturing:

Medium for PC3 cells:

RPMI 1640 (PAN, Aidenbach)

$10 \%$ FBS

$1.2 \%$ Penicillin/Streptomycin

Medium for VCaP cells:

DMEM, phenol red free (Gibco, Langenselbold)

$2 \%$ Sodium pyruvate $(100 \mathrm{mM})$

$1 \%$ L-glutamine

$10 \%$ FBS

$1.2 \%$ Penicillin/Streptomycin

Medium for T244, 2E and

Adv. DMEM (Gibco, Langenselbold)

BERKO/TRAMP 283 cells:

$10 \%$ FBS

$8 \%$ NuSerum

$80 \mu \mathrm{g} / \mathrm{ml}$ Gentamycine 


\section{$1 \%$ Glutamine \\ $100 \mathrm{nM}$ DHT}

Medium for establishment of

ProstaCult ${ }^{\text {TM }}$ Mouse Medium Kit (Stemcell murine

primary PCa cell lines: technologies, Cologne)

$10 \%$ FBS

$4 \mu \mathrm{g} / \mathrm{ml}$ Heparin

$10 \mathrm{ng} / \mathrm{ml}$ bFGF

$10 \mathrm{ng} / \mathrm{ml}$ EGF

$80 \mu \mathrm{g} / \mathrm{ml}$ Gentamycine

\subsubsection{Culture media for experiments}

Dextran-coated charcoal stripped FBS (stripped FBS) was used to supplement medium used for experiments. To generate stripped FBS, $50 \mathrm{ml}$ FBS were incubated with $1 \mathrm{~g}$ dextrancoated charcoal (Sigma-Aldrich, Taufkirchen) over night at $4^{\circ} \mathrm{C}$. Subsequently, charcoal was removed by centrifugation at $2000 \times \mathrm{g}$ for $10 \mathrm{~min}$. FBS was sterile filtered at kept at $-20^{\circ} \mathrm{C}$. Medium was supplemented with $10 \%$ stripped FBS.

\subsubsection{Culture media for bacteria}

LB (Luria Bertani)-Medium: $\quad \begin{array}{ll}10 \mathrm{~g} / \mathrm{l} \text { Bacto-Penton } \\ 5 \mathrm{~g} / \mathrm{l} \text { Yeast extract } \\ 10 \mathrm{~g} / \mathrm{NaCl} \\ \mathrm{pH} 7.0\end{array}$

Culture media were prepared with bi-distilled water, autoclaved and kept at RT. For clone selection ampicillin or kanamycin $(50 \mu \mathrm{g} / \mathrm{ml}$ final concentration) have been added to the media.

\subsubsection{Agar plates}

Before autoclaving $1.5 \%(\mathrm{w} / \mathrm{v})$ Agar-Agar was added to the liquid LB-medium. Medium was autoclaved and cooled down on a stirring plate to $55^{\circ} \mathrm{C}$ before $50 \mu \mathrm{g} / \mathrm{ml}$ ampicillin or kanamycin was added. Finally, the medium was poured into petri dishes and kept in a sterile plastic bag at $4^{\circ} \mathrm{C}$. 


\subsection{Biological Material}

\subsubsection{Bacterial Strains}

For the transformation of plasmids into competent bacterial strains, the Escherichia coli strain DH5a (Thermo Scientific, Langenselbold) was used.

\subsubsection{Eukaryotic cell lines}

In this study, the human adherent growing prostate cancer cell lines PC3 and VCaP and the murine adherent growing prostate cancer primary cell lines T244, 2E and BERKO/TRAMP 283 were used. Features of the cell lines important for this study are listed below.

PC3 cells

VCaP cells

2E cells

T244 cells

BERKO/TRAMP283 cells
Prostate adenocarcinoma cells (ATCC®; Rockville, USA) derived from bone metastatic site of a grade IV prostatic adenocarcinoma, no expression of the AR (androgen insensitive)

Prostate adenocarcinoma cells (LGC Standards Teddington, England) derived from vertebral metastatic site, derived from patient with hormone refractory prostate cancer, androgen sensitive

Murine prostate adenocarcinoma cells, isolated of a prostate tumor of a TRAMP mouse, isolated by this working group, C57/BI6 background

Murine prostate adenocarcinoma cells, isolated of a prostate tumor of a TRAMP mouse, isolated by this working group, C57/BI6 background

Murine prostate adenocarcinoma cells, isolated of a prostate tumor of a BERKO/TRAMP mouse, isolated by this working group, C57/BI6 background

\subsubsection{Mouse strains}

All mouse experiments were conducted according to European and German protection of animals act. The number of sacrificed mice as well as the stress and pain level was kept to a minimum. The mice were euthanized by $\mathrm{CO}_{2}$-asphyxation and cervical dislocation. Mice were 
kept under $12 \mathrm{~h}$ light/dark cycles at $22^{\circ} \mathrm{C}$ and $55 \pm 5 \%$ relative humidity. Animal food was ordered from ssniff Spezialdiäten $\mathrm{GmbH}$, Soest, Germany.

TRAMP (transgenic adenocarcinoma of mouse prostate) (Greenberg et al. 1995) and ER $\alpha$ knockout (ERKO) mice (Lubahn et al. 1993) with a C57/BI6 background were obtained from Jackson laboratories (Bar Harbor, USA). The ER $\beta$ knockout (BERKO) mice (Krege et al. 1998) with a C57/BI6 background were kindly provided by Jan-Åke Gustafsson, M.D., Ph.D., Director, Center for Nuclear Receptors and Cell Signaling, Department of Biology and Biochemistry, University of Houston, USA. Homozygous ERKO mice are subfertile. To generate mice with a homozygous ER a knockout and heterozygous for the TRAMP allele, the following crossing steps were performed: Male heterozygous ERKO mice were crossed with female heterozygous TRAMP mice. To generate mice with homozygous ER a knockout the offspring heterozygous for ERKO/TRAMP were crossed to heterozygous ERKO mice. Male homozygous BERKO mice were crossed with female heterozygous TRAMP mice to generate BERKO/TRAMP mice. TRAMP-FVB mice were bred from TRAMP mice with a C57/BI6 background and wild type mice with a FVB/N background.

\subsection{Synthetic DNA-Oligonucleotides}

Table 2.7: Human specific primers used for quantitative RT-PCR.

\begin{tabular}{l|l}
\hline Human-specific primers & Sequence \\
\hline DHCR_fw & CTGAAGACAAACCGAGAGGG \\
DHCR_rev & CATCCAGCCAAAGAGGTAGC \\
EAF2_fw & TCCCCAGCATCTCCAATAGA \\
EAF2_rev & TTTGGAATCTGATGAACTATCACAA \\
FDPS_fw & AGCCAAGGAAACAGGATGC \\
FDPS_rev & TCCATGATGTCATCTGCCAC \\
FGFR1_fw & GTCACAGCCACACTCTGCAC \\
FGFR1_rev & GGAAGGACTCCACTTCCACA \\
hlgf1 Q1 rev & AGGGGTGCGCAATACATCT \\
hAR-For-Q & AGGAACTCGATCGTATCATTGC \\
hAR-Rev-Q & CTCTGCCATCATTTCCGGAA \\
hlgf1 Q1 fw & TGGATGCTCTTCAGTTCGTG \\
hIGF1R-For-Q & CCGAAGGTCTGTGAGGAAGA \\
hIGF1R-Rev-Q & AATGGCGGATCTTCACGTAG \\
HPRT-fw & ACCCTTTCCAAATCCTCAGC \\
HPRT-rev & GTTATGGCGACCCGCAG \\
hPSA-For-Q & CCCCAGAATCACCCGAGCAG \\
hPSA-Rev-Q & TGAACCAGAGGAGTTCTTGAC \\
\hline
\end{tabular}




\begin{tabular}{l|l} 
IDI1_fw & CAGACTGTGCTCAAAGCGG \\
IDI1_rev & TGCTTGTCGAGGTGGTTAGT \\
IGFBP3-fw & GCCGTAGAGAAATGGAAGACACAC \\
IGFBP3-rev & CCATACTTATCCACACACCAGCAG \\
LDHA-fw & GGAGATCCATCATCTCTCCC \\
LDHA-rev & GGCCTGTGCCATCAGTATCT \\
MYB_fw & GCACCAGCATCAGAAGATGA \\
MYB_rev & CTTTCCACAGGATGCAGGTT \\
PTGER2_fw & GTCTGCTCCTTGCCTTTCAC \\
PTGER2_rev & TAAGGATGGCAAAGACCCAA \\
SHH_fw & CCAATTACAACCCCGACATC \\
SHH_rev & AGTTTCACTCCTGGCCACTG \\
SPOCK_fw & GACCGGGACAAGTACTGGAA \\
SPOCK_rev & CACACACACTTTGTGAGGGC \\
STAT6_fw & TCGCTGGACAGAGCTACAGA \\
STAT6_rev & CAGACCCCACAGAGACATGA \\
WNT2_fw & GAAGCCAACGAAAATGACC \\
WNT2_rev & CCCACAGCACATGACTTCAC
\end{tabular}

Table 2.8: Human specific primers used for PCR.

\begin{tabular}{l|l}
\hline Human-specific primers & Sequence \\
\hline GAPDH-fw & CATCACCATCTTCCAGGAGC \\
GAPDH-rev & ATGACCTTGCCCACAGCCTT \\
MMTV_rev_mittelstück & AGGGGCATCTGTTGGTCTTG \\
MMTV_rev_mittelstück2 & GTCCTTGGTGGGAAACACC \\
Seq MMTV-fw & GCAAAATAGGCTGTCCCCA \\
Seq MMTV-rev & AACATAAAGAAAGGCCCGGC \\
Vit-ERE Luc_fw & TCTGCTCTGATGCCGCATAG \\
Vit-ERE Luc_rev & GGAACCAGGGCGTATCTCTT
\end{tabular}

Table 2.9: Mouse specific primers used for genotyping PCR.

\begin{tabular}{l|l}
\hline Mouse-specific primers & Sequence \\
\hline ESR1wt-fw & GTAGAAGGCGGGAGGGCCGGTGTC \\
ESR1wt-REV & CGGTCTACGGCCAGTCGGGCA \\
ESR1mut-fw & GCCCTGAATGAACTGCAGGACG \\
ESR1mut-REV & CACGGGTAGCCAACGCTATGT \\
ESR2-MNeo25 & GCAGCCTCTGTTCCACATACACTTC
\end{tabular}


ESR2-MB1-2sp

ESR2-MC1wTnew

$\mathrm{rPb}-423-\mathrm{fw}$

TRAMP-SV40Tag
ATCAGCCCATGGGCAGAGTGTG

CCGTCATGACATAAGCATACCCT

CTCTGCACCTTGTCAGTGAGGTCCAG

CTCCTTTCAAGACCTAGAAGGTCCA

Table 2.10: Mouse specific primers used for quantitative RT-PCR.

\begin{tabular}{l|l}
\hline Mouse-specific primers & Sequence \\
\hline b-gluc-1-fw-1 & GCCTCCCAGAAGAAGACACC \\
b-gluc-1-fw-2 & GAGTGAATGGGAAGGGGTCG \\
b-gluc-1-rev 1 & CGACCCCTTCCCATTCACTC \\
b-gluc-1-rev 2 & GCTGTCACTGCCCAGAATCT \\
b-gluc-2-fw1 & ATCTGCTTGGCTCACGAGTT \\
b-gluc-2-rev1 & CTTTCGGTACCACCACTTCAA \\
mHPRT-For-Q & AGCCCCAAAATGGTTAAGGTTGC \\
mHPRT-Rev-Q & TTGCAGATTCAACTTGCGCTCAT \\
mTBP-Q-Fw & CACCAATGACTCCTATGACCCCTA \\
mTBP-Q-Rev & CAGTTGTCCGTGGCTCTCTTATTC \\
Rhodanase-fw1 & GTTCCAGCTGGTGGACTCTC \\
Rhodanase-rev1 & CATGAAGGGCATGTTGACTG
\end{tabular}

Table 2.11: Mouse specific primers used for PCR.

\begin{tabular}{l|l}
\hline Mouse-specific primers & Sequence \\
\hline Neo-berko_fw1 & TCTCCTGTCATCTCACCTTGC \\
Neo-berko_rev1 & GTCATTTCGAACCCCAGAGTC
\end{tabular}

\subsection{Antibodies}

\subsubsection{Inhibitory antibodies}

Table 2.12: Inhibitory antibodies and manufacturers

\begin{tabular}{l|l}
\hline Antibody & Company \\
\hline Rat IgG - Isotype Control & Abcam, Cambridge, UK \\
$\alpha$ Testosterone & Thermo Scientific, Langenselbold
\end{tabular}




\subsubsection{Primary antibodies}

Table 2.13: Primary antibodies and manufacturers

\begin{tabular}{l|l}
\hline Primary antibody & Company \\
\hline a AKT & Cell Signaling Technology Inc., Danvers, USA \\
a Androgen receptor & Lab Vision/NeoMarkers, \\
a cleaved caspase 3 & Cell Signaling Technology Inc., Danvers, USA \\
a ERK & Cell Signaling Technology Inc., Danvers, USA \\
a Estrogen receptor $\beta$ & Cell Signaling Technology Inc., Danvers, USA \\
a Estrogen receptor $\beta$ & Abcam, Cambridge, UK \\
a HSC70 & Santa Cruz, Heidelberg \\
a Ki-67 & BD Pharmingen, Franklin Lakes, USA \\
a pAKT & Cell Signaling Technology Inc., Danvers, USA \\
a pERK & Cell Signaling Technology Inc., Danvers, USA \\
a PSA & Cell Signaling Technology Inc., Danvers, USA \\
a SV40 Large T antigen & BD Pharmingen, Franklin Lakes, USA \\
a tubulin & Santa Cruz, Heidelberg
\end{tabular}

\subsubsection{Secondary antibodies}

Table 2.14: Secondary antibodies and manufacturers

\begin{tabular}{l|l}
\hline Secondary antibody & Company \\
\hline Goat-anti-rabbit IgG-HRP & Dianova, Hamburg \\
Goat-anti-mouse IgG-HRP & Dianova, Hamburg \\
Goat-anti-mouse IgG (whole & Sigma-Aldrich, Taufkirchen \\
molecule) F(ab') ${ }_{2}$-fragment-Cy3 & \\
Goat-anti-rabbit IgG (whole & Sigma-Aldrich, Taufkirchen \\
molecule) $F\left(a b^{\prime}\right)_{2}$-fragment-Cy3 &
\end{tabular}

\subsubsection{Blocking peptides}

Table 2.15: Blocking peptides and manufacturers

\begin{tabular}{l|l}
\hline Blocking peptide & Company \\
\hline Human ER $\beta$ peptide & Abcam, Cambridge, UK
\end{tabular}




\subsection{Plasmids and Vectors}

Table 2.16: Plasmids and vectors used in this study.

\begin{tabular}{l|l}
\hline Plasmid/Vector & Source \\
\hline MMTV-Luc reporter & kindly provided by Megan Cully, Cancer Research UK \\
PRL-Luc & $\begin{array}{l}\text { Promega, Fitchburg, USA } \\
\text { rPb-Luc reporter }\end{array}$ \\
& $\begin{array}{l}\text { carrying the minimal rat probasin promoter } \\
\text { kindly provided by Alo Nag, Feinberg School of } \\
\text { Vit-ERE-Luc reporter }\end{array}$ \\
& Medicine, Northwestern University
\end{tabular}

\subsection{Databases}

Table 2.17: Databases used in this study.

\begin{tabular}{|c|c|}
\hline Usage & Program \\
\hline Analysis of DNA sequences & $\begin{array}{l}\text { BLAST: Basic Local Alignment Search Tool, } \\
\text { www.ncbi.nlm.nih.gov/blast/ }\end{array}$ \\
\hline Analysis of statistically significance & $\begin{array}{l}\text { GraphPad, } \\
\text { http://graphpad.com/quickcalcs/ttest1.cfm }\end{array}$ \\
\hline Analysis of gene relationships & $\begin{array}{l}\text { DAVID Bioinformatics } \quad \text { Resources } \\
\text { https://david.ncifcrf.gov/ }\end{array}$ \\
\hline Comparison of gene sets & $\begin{array}{l}\text { Venny } 2.1 \text {, } \\
\text { http://bioinfogp.cnb.csic.es/tools/venny/ }\end{array}$ \\
\hline Genome Browser & Ensembl, http://www.ensembl.org/index.html \\
\hline Oligonucleotide design & Primer 3 Input, http://bioinfo.ut.ee/primer3/ \\
\hline Search for literature & $\begin{array}{l}\text { A plasmid editor, } \\
\text { http://biologylabs.utah.edu/jorgensen/wayned/ape/ } \\
\text { NCBI, http://www.ncbi.nlm.nih.gov/ }\end{array}$ \\
\hline
\end{tabular}

\subsection{Isolation, purification and concentration measurement of nucleic acids}

\subsubsection{Isolation of DNA from mouse tail}

The DNA template for the genotyping PCR was extracted from mouse tails by incubating the tails with $200 \mu \mathrm{l} \mathrm{PCR}$ direct (Peqlab, Erlangen) and proteinase $\mathrm{K}$ (Carl Roth $\mathrm{GmbH}$, Karlsruhe) at $55^{\circ} \mathrm{C}$ overnight. The reaction was terminated by heating the solution up to $85^{\circ} \mathrm{C}$ for $45 \mathrm{~min}$. 


\subsubsection{DNA purification with DNA binding columns}

Smarter Nucleic Acid Sample Preparation columns (Stratec, Berlin) were used to purify DNA. Up to $50 \mu \mathrm{l}$ DNA solution was mixed with $500 \mu \mathrm{l}$ binding buffer and loaded into a DNA binding column. The column was centrifuged for $3 \mathrm{~min}$ at $12.000 \mathrm{xg} \cdot 10-50 \mu \mathrm{l} \mathrm{H}_{2} \mathrm{O}$ were loaded to the column and incubated for $5 \mathrm{~min}$ at RT. The column was centrifuged for $1 \mathrm{~min}$ at $12.000 \times \mathrm{g}$. The flow through contained the purified DNA and could be stored at $-20^{\circ} \mathrm{C}$.

\subsubsection{RNA purification of cell cultures}

Total RNA from cell cultures was isolated and purified using the peqGold Total RNA Kit (Peqlab, Erlangen) according to the manufacturer's instructions.

\subsubsection{RNA purification of mouse tissue}

Total RNA from mouse tissue was isolated and purified using the peqGold Total RNA Kit (Peqlab, Erlangen). Small tissue samples were transferred into $2 \mathrm{ml}$ tubes with $400 \mu \mathrm{l}$ lyses buffer. A stainless steel bead (5 $\mathrm{mm}$ in diameter, Quiagen, Hilden) was added and tissue sections were homogenized by the TissueLyser LT (Quiagen, Hilden) at max. speed for 5 min. Further proceedings were performed according to the manufacturer's instructions.

\subsubsection{Determination of DNA and RNA concentration}

For quantification of nucleic acids, the Nanodrop (Thermo Scientific, Langenselbold) was used. This device measures DNA or RNA content of samples by light absorption. Furthermore, the Nanodrop gives information about the purity of a sample by showing absorption at other wavelengths.

To measure nucleic acid concentration, $1 \mu \mathrm{l}$ of the sample was appended on the spectrophotometer. The Nanodrop measured absorption at $260 \mathrm{~nm}$ for DNA and RNA and calculated RNA or DNA concentration.

\subsection{Whole mRNA-sequencing analysis}

A whole mRNA-sequencing analysis was used to identify the differentially expressed genes in VCaP rev., VCaP and VCaP AA cells with or without $8 \beta$-VE2 treatment. Therefore, the Illumina detection system (San Diego, USA) was used. The analysis was performed in cooperation with the TAL facility of the Georg-August-University Göttingen.

\subsubsection{RNA isolation}

All working materials and surfaces were cleaned with RNaseZAP® (Sigma-Aldrich, Taufkirchen) to remove free RNase. VCaP variants were treated with $25 \mu \mathrm{M} 8 \beta$-VE2 or 
DMSO as control for $24 \mathrm{~h}$. Then, cells were detached by treatment with trypin/EDTA. Cells were washed twice in DPBS and resuspended in TRIzol® (Thermo Scientific, Langenselbold). 0.2 Vol Chloroform was appended and vortexed thoroughly for $15 \mathrm{sec}$. The solution was incubated for $5 \mathrm{~min}$ at RT and centrifuged at $12.000 \mathrm{xg}$ at $4^{\circ} \mathrm{C}$ for $15 \mathrm{~min}$. The upper phase was transferred into a fresh tube and supplemented with $0.5 \mathrm{ml}$ isopropanol. After vortexing thoroughly, the solution was incubated at $-20^{\circ} \mathrm{C}$ over night. The sample was centrifugated at $12.000 \times \mathrm{g}$ at $4^{\circ} \mathrm{C}$ for $30 \mathrm{~min}$ and the supernatant was removed and $1 \mathrm{ml}$ ethanol was appended and centrifugated for $5 \mathrm{~min}$ at $12.000 \mathrm{xg}$ at $4^{\circ} \mathrm{C}$. The supernatant was discarded and this washing step was repeated twice. The RNA pellet was air dried and dissolved in $100 \mu \mathrm{lddH_{2 }} \mathrm{O}$. RNA was stored at $-80^{\circ} \mathrm{C}$.

\subsubsection{Generation of the Illumina cDNA library}

To generate an RNA library the TruSeq RNA Library Preparation Kit v2 (Illumina, San Diego, USA) was used. For library preparation, mRNA was enriched by coupling to bead tagged poly-(dT) oligos and subsequently, mRNA was fragmented by the Bioruptor NGS (Diagenode, Liège, Belgium) and primed with random hexamers. RNA fragments were transcribed into cDNA and 3' ends were adenylated; 5' ends phosphorylated. Adaptor oligonucleotides were ligated to the cDNA fragments, allowing the sequencing of the fragments. Finally, fragments are amplified for the cDNA library (see Fig. 2.1). 


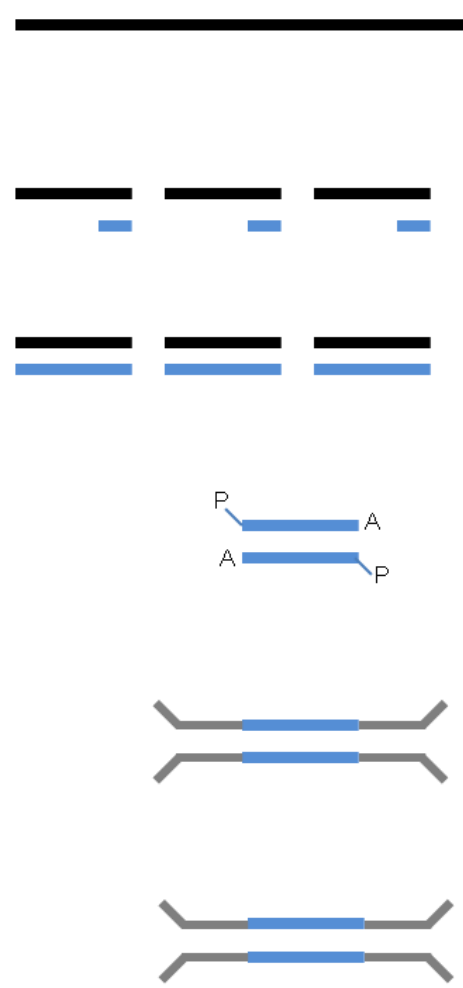

Enrichment of mRNA using

poly-(dT)-coupled beads

RNA fragmented and

primed

First strand cDNA

synthesis

$3^{\prime}$ ends adenylated and 5'

ends repaired

DNA sequencing adaptors

ligated

Amplification of ligated

fragments

Figure 2.1: cDNA library preparation. To perform efficient whole mRNA-sequencing analysis, a cDNA library was generated. Therefore, mRNA was enriched by isolation via poly-(dT) coupled beads. RNA was fragmented and annealed to random hexamers. cDNA was transcribed. Adapters, carrying primer binding sites were ligated to the cDNA. PCR is used to amplify fragments. Modified according to http://www.illumina.com/products/scriptseq-rna-seq-library-prep.html

\subsubsection{Sequencing of cDNA}

Illumina detection system uses sequencing by synthesis employing fluorescent-based reversible sequence termination. cDNA fragments were appended on a flow cell. Here, the adapter was coupled to the cDNA fragments hybridized to the oligonucleotides on the flow cell surface. cDNA fragments were clonally amplified to increase signal intensity. Then, fluorescent tagged dNTP's were incorporated. DNTP's were reversible terminators, meaning only one nucleotide per cycle was incorporated. Every nucleotide (A, T, C, G) carried a specific dye, so fluorescent signal gives position information about the incorporated nucleotide (Fig. 2.2). Analyses were performed using the HiSeq 2000 (Illumina San Diego, USA) (SR; $1 \times 50$ bp; app. 30 Mio reads per sample). Sequence images were transformed to bcl files using Illumina software BaseCaller, which were demultiplexed to fastq files with CASAVA v1.8.2 and quality checks were done via fastqc. Reads were aligned using STAR v2.3.0 to the hg19 reference genome and data conversion and sorting was carried out with samtools 0.1.191 and reads per gene were counted via htseq version 0.6.12. Gene expression was calculated by fragments per kilobase per million reads (FPKM). Data analysis was performed using R/Bioconductor (3.0.2/2.123) with DESeq2 and gplots 
packages. Candidate genes were filtered to a minimum of false discovery rate (FDR)corrected $p$-value $<0.05$. As threshold for differentially expressed genes $\log _{2}$-fold was set.

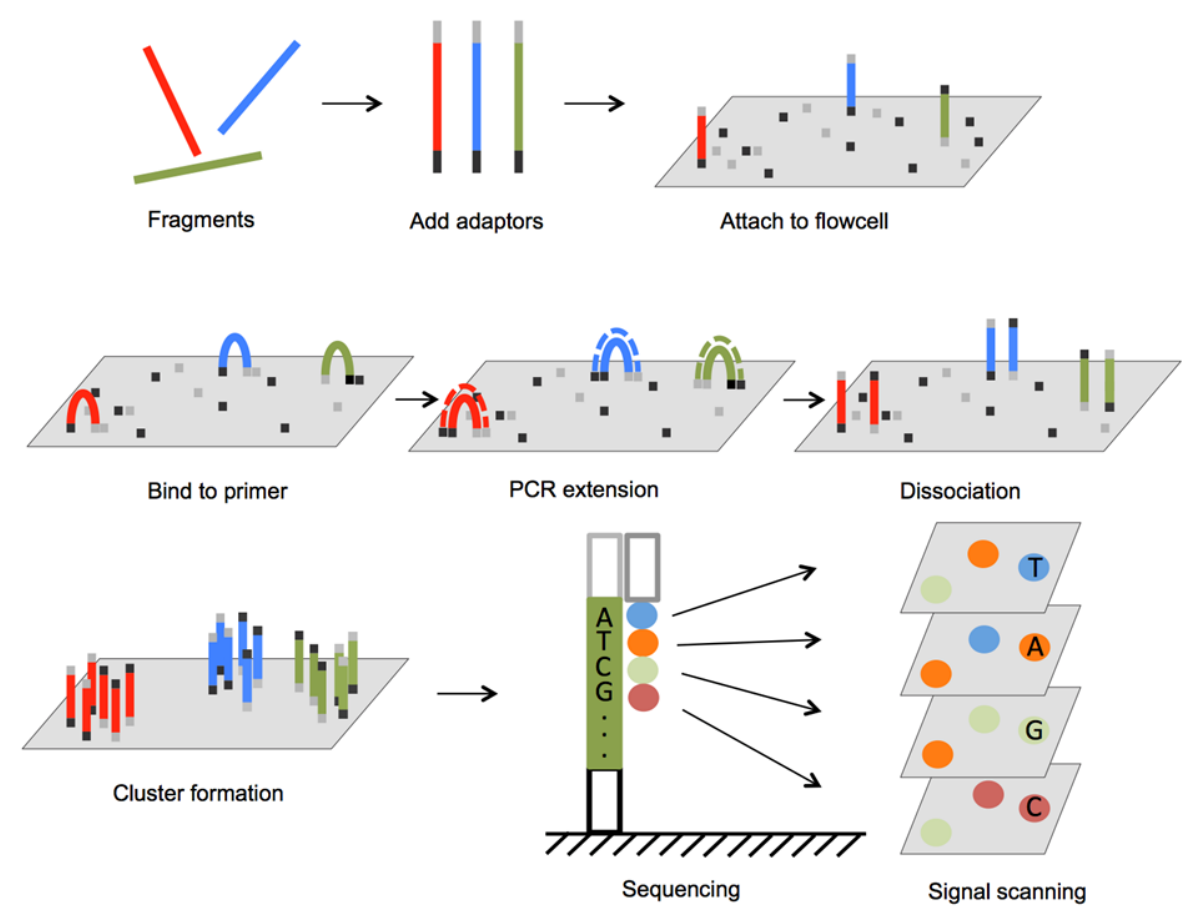

Figure 2.2: Sequence detection by synthesis. CDNA fragments are coupled to adaptor oligonucleotides that can attach to the flow cell. By PCR clonal clusters are generated to increase signal intensity during sequencing with differently labeled dNTPs. Modified according to Kulski (2016).

The in silico upstream target analysis was calculated using the following algorithm (Kel et al. 2006):

$$
S(X)=\frac{\frac{\sum_{r=1}^{R} M(X, r)}{M \max (r)}}{1+p N(X, r) / N \max (r)}
$$

$\mathrm{R}=$ Max radius (input parameter $=8$ )

$\mathrm{p}=$ Penalty (input parameter)

$N(X, r)=$ Total number of molecules reachable from key molecule $X$ within the radius $r$

$\mathrm{Nmax}(r)=$ maximal value for $N(X, r)$ over all key molecule $X$ found for this radius

$M(X, r)=$ Sum of $w(X)$ for all hits reachable from key molecule $X$ within the radius $r$, where $w(X)$ - weight of hits $X$.

$\operatorname{Mmax}(r)=$ Maximal value of $M(X, r)$ over all key molecules $X$ found for this radius. 


\subsection{Cloning techniques}

\subsubsection{Vector preparation}

[Birnboim et al. 1979]

Vector preparation is used to purify vectors from bacterial cells. The cells are lysed and after removing proteins and other contaminates the DNA is precipitated.

The vector preparation with the Plasmid-Midi Kit (Thermo Scientific, Langenselbold) was performed according to the manufacturer's instructions.

\subsubsection{Establishment of bacterial glycerol stocks}

For storage of transformed E. coli bacteria glycerol stocks were generated. $1 \mathrm{ml}$ overnight culture was supplemented with $100 \mu \mathrm{l}$ glycerol (Carl Roth $\mathrm{GmbH}$, Karlsruhe) and carefully vortexed. The bacteria stock was stored at $-80^{\circ} \mathrm{C}$.

\subsubsection{Transformation of DH5a Escherichia coli bacteria}

[Hanahan et al. 1983]

Transformation describes the process of embedding former DNA, e.g. vectors, into E. coli bacteria. E. coli reproduce and multiply the embedded DNA.

$1 \mu \mathrm{l}$ vector DNA was added to $50 \mu \mathrm{l}$ of competent DH5a E. coli bacteria cells. Bacteria were incubated for $30 \mathrm{~min}$ on ice. Then, $E$. coli cells were heated for $90 \mathrm{sec}$ at $42^{\circ} \mathrm{C}$. The bacteria cells were placed on ice for $5 \mathrm{~min}$. Next, $200 \mu \mathrm{LB}$ medium was added to the bacteria cells. The E. coli cells were shook horizontally for $1 \mathrm{~h}$ at $37^{\circ} \mathrm{C}$. Subsequently, the bacteria culture was plated onto an antibiotic containing LB-agar plate to select vector carrying cells. The plate was incubated for $16 \mathrm{~h}$ at $37^{\circ} \mathrm{C}$.

\subsection{Gel electrophoresis}

Agarose gel electrophoresis is used to separate nucleic acid molecules with different sizes. In this study, $0.7 \%-1 \%$ agarose gels boiled with $1 \times$ Turbo buffer were used. Stain G (Serva, Heidelberg), diluted 1:20.000 to 1:50.000, was applied to the liquid gel. The samples were mixed with loading buffer in a 4:1 ratio and loaded into the gel pockets. A 1 kb Plus DNAladder (Thermo Scientific, Langenselbold) was used for the determination of DNA size. The DNA was separated with $100 \mathrm{~V}$ and $150 \mathrm{~mA}$. To visualize the signal Stain $\mathrm{G}$ was excited with UV light. 


\subsection{Polymerase chain reaction}

[Saiki et al. 1985]

Polymerase chain reaction (PCR) is used to amplify DNA. For PCR the DNA is denatured to separate double stranded DNA into single strands. In the following annealing phase the DNA and primers bind to each other. In the elongation step the DNA polymerase binds to the DNA-primer complex and amplifies the DNA. These three steps were repeated in one PCR so that the target sequence is enriched exponentially.

\subsubsection{Amplification of DNA fragments}

For DNA fragment amplification the Mango Taq $^{\text {TM }}$ (Bioline, Luckenwalde) was used for PCR. $25 \mu \mathrm{l}$ of PCR solution contained:

Final concentration

$\mathrm{X} \mu \mathrm{l}$ template DNA (10-50 $\mathrm{ng})$

$10 \mu l 5 x$ Buffer

$1 \mathrm{x}$

$1.25 \mu \mathrm{l} 2 \mathrm{mM}$ dNTPs

$200 \mu \mathrm{M}$ each

$2.5 \mu \mathrm{l}$ of Primer 1 (10 pmol/ $\mu \mathrm{l}$ sequence specific)

$0.5 \mu \mathrm{M}$

$2.5 \mu$ of Primer 2 (10 pmol/ $\mu$ l sequence specific)

$0.5 \mu \mathrm{M}$

$1 \mu$ l Mango Taq $^{\mathrm{TM}}$ DNA Polymerase

$0.04 \mathrm{U} / \mu \mathrm{l}$

add to $25 \mu \mathrm{l} \mathrm{H}_{2} \mathrm{O}$

PCR program was adjusted to the fragment length. A standard PCR program would be:

\begin{tabular}{|c|c|c|}
\hline $95^{\circ} \mathrm{C}$ for $3 \mathrm{~min}$ & & first denaturation \\
\hline $95^{\circ} \mathrm{C}$ for $30 \mathrm{sec}$ & & denaturation \\
\hline $62^{\circ} \mathrm{C}$ for $30 \mathrm{sec}$ & $25-40$ times & annealing \\
\hline $72^{\circ} \mathrm{C}$ for $1 \mathrm{~min}$ & & elongation \\
\hline $72^{\circ} \mathrm{C}$ for $7 \mathrm{~min}$ & & final elongation \\
\hline
\end{tabular}

The denaturation temperature depends mainly on the used polymerase. The annealing temperature can vary depending on the primer pair and the elongation time mainly depends on the fragment size and the amplification speed of the polymerase.

\subsubsection{DNA-sequencing according to Sanger}

[Sanger et al. 1977]

DNA sequencing was used to identify mutations and screen for sequence aberrations. Therefore, the chain termination principle according to Sanger was used. Differently fluorescent labeled dideoxynucleotides were used for the sequence reaction. In combination with normal deoxynucleotides the DNA chain is replicated until randomly a dideoxynucleotide 
is integrated and leads to the chain termination. The fluorescent labeling can be detected and gives information about the nucleotide sequence.

For the analyses the BigDye® system (Thermo Scientific, Langenselbold) was used.

$10 \mu \mathrm{l}$ reaction solution contained:

$1 \mu$ BigDye® Master Mix

$2 \mu \mathrm{l} 5 \mathrm{x}$ buffer

$1 \mu \mathrm{l}$ sequence specific primer $(10 \mathrm{pmol} / \mu \mathrm{l})$

$\mathrm{X} \mu \mathrm{l}$ DNA template (100 $\mathrm{ng} / 100 \mathrm{bp}$ length)

add up to $10 \mu$ with $\mathrm{ddH}_{2} \mathrm{O}$

The following sequencing program was used:

$\left.\begin{array}{ll}95^{\circ} \mathrm{C} & 1 \mathrm{~min} \\ 95^{\circ} \mathrm{C} & 30 \mathrm{sec} \\ 60^{\circ} \mathrm{C} & 3 \mathrm{~min} \\ 60^{\circ} \mathrm{C} & 5 \mathrm{~min}\end{array}\right\} 30$ cycles

Analyses of sequences were performed by gel electrophoretic separation though specific capillaries. Here, the fluorescent dyes were detected by a laser. The gel electrophoretic analysis was carried out in the $\mathrm{ABI} 3500 \mathrm{XL}$ genetic analyzer (Applied biosystems, Darmstadt).

\subsubsection{Reverse Transcription}

Reverse transcription is used to transcribe mRNA into cDNA. Here, the Reverse Transcriptase SuperScript II (Thermo Scientific, Langenselbold) was used. The SuperScript II binds together with poly(dT)-primer to the poly-A region of mRNA and synthesize cDNA templates.

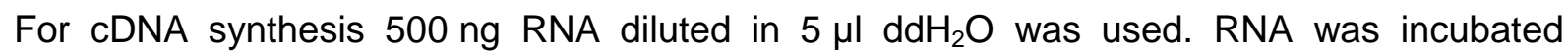
together with $0.5 \mu \mathrm{l} 10 \mathrm{mM}$ dNTPs and $0.5 \mu \mathrm{l}$ poly(dT) primer for $10 \mathrm{~min}$ at $72^{\circ} \mathrm{C}$ and $2 \mathrm{~min}$ at $42^{\circ} \mathrm{C}$. $2 \mu \mathrm{l} 5 \times$ SuperScript II Buffer and $1 \mu \mathrm{I}$ DTT were added and incubated at $42^{\circ} \mathrm{C}$ for $2 \mathrm{~min}$. $0.25 \mu \mathrm{l}$ SuperScript II diluted in $0.75 \mu \mathrm{lddH_{2 }} \mathrm{O}$ were added and incubated for 50 min at $42^{\circ} \mathrm{C}$. Finally, the polymerase was heat-inactivated at $72^{\circ} \mathrm{C}$ for $10 \mathrm{~min}$. Success of cDNA transcription was verified by GAPDH PCR (see 2.17.1).

\subsubsection{Quantitative real time PCR}

The quantitative real time PCR (qRT-PCR) analysis enables the quantification of mRNA content of chosen genes in a sample. The qRT-PCR experiment utilizes the principles of PCR together with incubation of a fluorescent dye. The amount of PCR product is proportional to the fluorescent signal and allows calculation of template concentration. 
In the present study the PCR Mastermix Platinum SYBR Green qPCR SuperMix-UDG with Rox (Thermo Scientific, Langenselbold) was used. SYBR Green, which binds double stranded DNA can be excited using light of $480 \mathrm{~nm}$ wavelength and shows an emission spectrum with a maximum at $520 \mathrm{~nm}$. The Rox dye is used as reference to correct pipetting errors. The fluorescent intensity was measured after every PCR cycle using the ABI Prism 7900T Sequence Detection System. During the exponential phase of the PCR reaction conditions are optimal (e.g. maximum polymerase activity and optimal ratio of reaction materials such as primer and $\mathrm{MgCl}_{2}$ ). During this phase the threshold value is determined. The threshold value (Ct value) was used for further calculations for quantification. Data was evaluated with the Sequence-detection system software (SDS Version 2.1, PE Applied biosystems, Darmstadt).

The cDNA was diluted 1:20 in $\mathrm{ddH}_{2} \mathrm{O}$.

The qRT-PCR reaction was comprised of the following components:

\section{$2.5 \mu \mathrm{l}$ cDNA}

$2.5 \mu \mathrm{l}$ Primer (Fw + Rev $100 \mathrm{pmol} / \mu \mathrm{l})$

$5 \mu \mathrm{l}$ SYBR Green

The program on the ABI Prism 7900T Sequence Detection System was the following:

$\left.\begin{array}{ll}50^{\circ} \mathrm{C} & 2 \mathrm{~min} \\ 95^{\circ} \mathrm{C} & 3 \mathrm{~min} \\ 94^{\circ} \mathrm{C} & 15 \mathrm{sec} \\ 60^{\circ} \mathrm{C} & 30 \mathrm{sec} \\ 72^{\circ} \mathrm{C} & 30 \mathrm{sec} \\ 95^{\circ} \mathrm{C} & 15 \mathrm{sec} \\ 60^{\circ} \mathrm{C} & 15 \mathrm{sec} \\ 60^{\circ} \mathrm{C}-95^{\circ} \mathrm{C} & 2^{\circ} \mathrm{C} / \mathrm{min}\end{array}\right\} \begin{array}{ll}\text { Taq activation } \\ 40 \mathrm{x}\end{array}$

After measurement the data was transferred to MS Excel (Microsoft) for further calculations. Relative expression was determined by the $\Delta \Delta$-Ct-method, which uses the following formula: $\Delta \mathrm{Ct}=\mathrm{Ct}$ (Gene of interest) $-\mathrm{Ct}$ (housekeeping gene) $\Delta \Delta \mathrm{Ct}=\Delta \mathrm{Ct}$ (Control) $-\Delta \mathrm{Ct}$ (gene of interest) Relative expression $=2 \Delta \Delta \mathrm{Ct}$

The mRNA expression of the housekeeping genes Hypoxanthin-GuaninPhosphoribosyltransferase (HPRT) and Lactate dehydrogenase A (LDHA) was used as reference. 


\subsection{Protein chemical techniques}

\subsubsection{Isolation of protein from cell culture}

Protein was isolated from cell culture by incubation of cells with modified RIPA buffer. For cell culture $100 \mu \mathrm{l}$ modified RIPA buffer were appended on a 6-well plate. Samples were incubated for $10 \mathrm{~min}$ on ice and transferred into a reaction tube. To purify the protein lysate and remove cell debris the samples were centrifuged for $10 \mathrm{~min}$ at $13.000 \mathrm{xg}$ at $4^{\circ} \mathrm{C}$ and the supernatant was transferred into a new cup. Protein was stored at $-20^{\circ} \mathrm{C}$.

\subsubsection{Isolation of protein from mouse tissue}

Protein was isolated from mouse tissue by using modified RIPA buffer. Small tissue samples were transferred into $2 \mathrm{ml}$ tubes with $150 \mu$ modified RIPA buffer. A stainless steel bead ( $5 \mathrm{~mm}$ in diameter) was added and tissue sections were homogenized by the TissueLyser LT at max. speed for $5 \mathrm{~min}$. To purify the protein lysate and remove cell debris the samples were centrifuged for $10 \mathrm{~min}$ at $13.000 \mathrm{xg}$ at $4^{\circ} \mathrm{C}$ and the supernatant was transferred into a new cup. Protein was stored at $-20^{\circ} \mathrm{C}$.

\subsubsection{Determination of protein concentration}

[Bradford 1976]

Protein concentration was measured using the Roti ${ }^{\circledR}$-Nanoquant (Carl Roth $\mathrm{GmbH}$, Karlsruhe) reagent. The reagent contains Coomassie Brilliant Blue G-250 that binds to the cationic and nonpolar hydrophobic side chains of proteins. This initiates a change of the absorption maximum from $365 \mathrm{~nm}$ to $595 \mathrm{~nm}$. The increase of the absorption for $595 \mathrm{~nm}$ compared to $\mathrm{H}_{2} \mathrm{O}$ control is proportional to the concentration of proteins.

Protein lysate was diluted $1: 100$ in $\mathrm{H}_{2} \mathrm{O} .50 \mu \mathrm{l}$ of this solution were appended into a translucent 96-well plate. The measurement was performed in triplicates. $200 \mu$ l Nanoquant solution was applied into each well and incubated five minutes before absorption was measured with the Synergy Mx plate reader (BioTek, Winooski, USA). 10 to $15 \mu$ g protein were used for western blot analysis.

\subsubsection{SDS-Polyacrylamide gel electrophoresis (SDS-PAGE)}

Proteins were separated depending on their molecular weight with the NuPAGE® Pre-Cast Gel System (Thermo Scientific, Langenselbold). Therefore, the proteins were denatured by heating and negatively charged by incubation with SDS (sodium dodecyl sulfate). The proteins were separated using gel electrophoresis.

Protein lysate was supplemented with NuPAGE® LDS Sample Buffer (4x) (Thermo Scientific, Langenselbold) with $10 \%$ DTT (Biomol, Hamburg) and boiled for $10 \mathrm{~min}$ at $70^{\circ} \mathrm{C}$. The samples were centrifuged for 1 min and loaded onto the NuPAGE ${ }^{\text {TM }} 4-12 \%$ Bis-Tris gel 
(Thermo Scientific, Langenselbold). As a marker for protein size NuPAGE ${ }^{\text {TM }}$ See Blue Plus2 (Thermo Scientific, Langenselbold) was used. Gel electrophoresis was performed for $1.5 \mathrm{~h}$ with $160 \mathrm{~V}$ and $160 \mathrm{~mA}$ in MES-Buffer (Thermo Scientific, Langenselbold).

\subsubsection{Western blotting of proteins onto Polyvinylidene Difluoride membranes}

[Gershoni, Palade 1982]

For signal detection proteins had to be transferred onto a Polyvinylidene Difluoride (PVDF) membrane (GE Healthcare, Freiburg) by semi-dry blotting. The negatively charged proteins were electro-blotted onto the membrane.

The PVDF membrane was equilibrated together with six whatman filter papers (Schleicher \& Schüll, Dassel) for 10 min in transfer buffer lla. For the blotting procedure three whatman filter papers, the PVDF membrane, the SDS-Page gel and again three whatman filter papers were placed onto the anode of the electro-blotter (Biometra, Göttingen). Finally, the cathode was placed on top. The blotting was performed at $25 \mathrm{~V}$ and $225 \mathrm{~mA}$ for 25-35 min.

\subsubsection{Incubation of protein-bound membranes with antibodies}

To block all unspecific binding sites the membrane was incubated with blocking buffer for $2 \mathrm{~h}$. The membrane was washed with TBS/T and incubated with the primary antibody specific against the protein of interest in TBS $/ \mathrm{T}$ at $4^{\circ} \mathrm{C}$ overnight. The following day the membrane was washed twice for 10 min with washing buffer. Then, the membrane was incubated for $2 \mathrm{~h}$ with the horseradish peroxidase (HRP) labeled secondary antibody specific against the donor species of the primary antibody. Afterwards, the membrane was washed in washing buffer three times for $15 \mathrm{~min}$. To detect the signal the ECL Prime Kit (GE Healthcare, Freiburg) was used according to the manufacturer's instructions. After $20 \mathrm{sec}$ to 4 min incubation the signal was detected by the western blot detection system FlourChem ${ }^{\circledR}$ Q Alpha (Alpha Innotech, Logan, Utah, USA).

Table 2.18: Antibodies used for western blot analysis. Listed are primary and secondary antibodies used for western blot analysis. Primary antibodies were diluted in TBS/T, secondary antibodies were diluted in blocking buffer.

\begin{tabular}{l|c|c|c}
\hline Primary antibody & $\begin{array}{l}\text { Dilution primary } \\
\text { antibody }\end{array}$ & $\begin{array}{l}\text { Dilution secondary } \\
\text { antibody }\end{array}$ & $\begin{array}{l}\text { Blocking reagent } \\
\text { in TBS/T }\end{array}$ \\
\hline $\begin{array}{l}\text { a AKT, monoclonal } \\
\text { antibody, rabbit }\end{array}$ & $1: 2000$ & $1: 20.000$ & $5 \%$ powder milk \\
$\begin{array}{l}\text { a AR, monoclonal } \\
\text { antibody, rabbit }\end{array}$ & $1: 4000$ & $1: 10.000$ & $5 \%$ powder milk \\
\end{tabular}




\begin{tabular}{|c|c|c|c|}
\hline $\begin{array}{l}\alpha \quad \text { ER } \beta, \text { polyclonal } \\
\text { antibody, rabbit (Cell } \\
\text { Signaling) }\end{array}$ & $1: 10.000$ & $1: 10.000$ & $3 \%$ BSA \\
\hline $\begin{array}{l}\alpha \quad \text { ER } \beta, \text { polyclonal } \\
\text { antibody, rabbit (Abcam) }\end{array}$ & $1: 1000$ & $1: 10.000$ & $5 \%$ powder milk \\
\hline $\begin{array}{l}\alpha \quad \text { ERK, polyclonal } \\
\text { antibody, rabbit }\end{array}$ & $1: 2000$ & $1: 20.000$ & $5 \%$ powder milk \\
\hline $\begin{array}{l}\text { a HSC70, monoclonal } \\
\text { antibody, mouse }\end{array}$ & $1: 10.000$ & $1: 10.000$ & $5 \%$ powder milk \\
\hline $\begin{array}{l}\text { a pAKT, monoclonal } \\
\text { antibody, rabbit }\end{array}$ & $1: 2000$ & $1: 20.000$ & $5 \%$ powder milk \\
\hline $\begin{array}{l}\text { a pERK, monoclonal } \\
\text { antibody, rabbit }\end{array}$ & $1: 2000$ & $1: 20.000$ & $5 \%$ powder milk \\
\hline $\begin{array}{l}\text { a Tubulin, monoclonal } \\
\text { antibody, mouse }\end{array}$ & $1: 20.000$ & $1: 40.000$ & $5 \%$ powder milk \\
\hline $\begin{array}{l}\text { a PSA, monoclonal } \\
\text { antibody, rabbit }\end{array}$ & $1: 4000$ & $1: 10.000$ & $5 \%$ powder milk \\
\hline
\end{tabular}

\subsection{Cell biological methods}

\subsubsection{Culturing of eukaryotic cells}

The human prostate cancer cell lines PC3 and VCaP and the murine primary prostate cancer cell lines T244, 2E and BERKO/TRAMP 283 were used in this study. PC3 cells were cultured in RPMI medium (PAN, Aidenbach) supplemented with $10 \%$ FBS (PAN, Aidenbach) and $1.2 \%$ penicillin/streptomycin (P/S) (PAN, Aidenbach). The three VCaP cells variants, VCaP rev., VCaP and VCaP AA, were used for the experiments. VCaP cells were cultured in DMEM medium (Gibco, Langenselbold) supplemented with $10 \%$ FBS, 2 \% sodium pyruvate (Gibco, Langenselbold), $1.2 \%$ (P/S) and $1 \%$ L-glutamine (PAN, Aidenbach). VCaP rev. cells were grown in DMEM medium further supplemented with $1 \mathrm{nM}$ testosterone (Sigma-Aldrich, Taufkirchen) for seven month before use. VCaP AA cells were cultured in DMEM medium with rising concentration of abiraterone acetate (up to $5 \mathrm{mM}$, Janssen Cilag, Neuss) for three weeks and further cultivated in medium containing the agent. T244, 2E and BERKO/TRAMP 283 cells were cultured in Adv. DMEM medium (Gibco, Langenselbold) supplemented with $10 \%$ FBS, 8 \% NuSerum (Corning Life Sciences, Bedford, USA), 100 nM DHT (Sigma-Aldrich, Taufkirchen) and $1.2 \% \mathrm{P} / \mathrm{S}$.

The cells were grown in a humidified atmosphere at $37^{\circ} \mathrm{C}$ and with $5 \% \mathrm{CO}_{2}$ and allowed to grow until they reached $70-90 \%$ confluence. Then, cells were split by incubation with trypsin/EDTA (PAN, Aidenbach) to dissect the adherence. The proteolytic reaction was 
terminated by adding growth medium. For experimental usage specific numbers of cells were used. Therefore, the cells were counted with a Neubauer improved counting chamber (Hartenstein, Wuerzburg).

\subsubsection{Generation of murine primary cell culture}

Primary cell lines were generated from PCa tumors of TRAMP and BERKO/TRAMP mice to analyze the effect of ER $\beta$ for PCa growth and progression.

From the age of 16 weeks on male TRAMP and BERKO/TRAMP mice were under surveillance. PCa growth was examined at least once a week by palpation of the lower abdominal area. When tumor size $>1 \mathrm{~cm}^{3}$ was reached, mice were sacrificed. The tumor was harvested and washed with DPBS. Further proceedings were performed under sterile conditions. Tumor tissue was manually chopped using a sterile scalpel blade and washed with DPBS again. Cells were centrifugated for $5 \mathrm{~min}$ at $1000 \mathrm{xg}$ and incubated for at least $30 \mathrm{~min}$ in $1 \mathrm{mg} / \mathrm{ml}$ collagenase dissolved in HBSS. Cells were centrifugated again and washed with HBSS twice. Then, cells were incubated with trypsin for $5 \mathrm{~min}$ at $37^{\circ} \mathrm{C}$ and subsequently washed in HBSS once and appended on a cell strainer (BD Biosciences, Heidelberg). Flow through was pelleted and plated in ProstaCult medium (Stemcell technologies, Cologne) into a fibronectin or Matrigel® Basement Membrane Matrix (Corning Inc., New York, USA) coated cell flask.

\subsubsection{Freezing and thawing of eukaryotic cells}

Human eukaryotic cancer cell lines were kept in a $10 \%$ DMSO-medium solution at $-196^{\circ} \mathrm{C}$ in liquid nitrogen for longer storage. For murine primary cells a freezing solution of $20 \%$ DMSO, $30 \% \mathrm{FBS}$ and $50 \%$ medium was used. Cells were thawed in a $37^{\circ} \mathrm{C}$ warm water bath, taken up with $5 \mathrm{ml}$ fresh medium and centrifuged at $200 \mathrm{xg}$ for $10 \mathrm{~min}$. The supernatant was discarded and the cells were resuspended with fresh growing medium and transferred into a culture flask. Cells were allowed to grow at $37^{\circ} \mathrm{C}$ in a humidified atmosphere with $5 \% \mathrm{CO}_{2}$.

\subsubsection{Test for mycoplasma spec.}

Mycoplasma are bacteria that infest cell culture and affect cell function and activity (Drexler, Uphoff 2002). Every four to six weeks a mycoplasm spec. test was performed to ensure the cell reactions were not altered due to mycoplasm toxification.

The MycoAlert® Mycoplasma Detection Kit (Lonza, Cologne) was used to detect mycoplasm specific enzymes in culture medium. By adding the substrate, the enzymes catalyze the reaction of ADP to ATP. The concentration of ATP is measured via a luminescence reaction:

$$
\mathrm{ATP}+\text { Luciferin }+\mathrm{O}_{2} \rightarrow \text { Oxiluciferin }+\mathrm{AMP}+\mathrm{PP}_{\mathrm{i}}+\mathrm{CO}_{2}+\text { light }
$$


ATP concentration is linear correlated to severity of mycoplasma contamination.

Test for mycoplasma spec. was performed according to the manufacturer's instructions. Luminescence was measured with the Synergy Mx. Contaminated cells were discarded.

\subsubsection{Transfection of eukaryotic cells}

To generate cells with ectopic gene expression cells were transfected with expression vectors. For transfection, reagents composed of lipids that form complexes with vector DNA are used. These complexes are able to pass the cell membrane. For transfection, $70-90 \%$ confluent cells were incubated with the transfection reagent-DNA complex. The transfection reagents Lipofectamine ${ }^{\circledR} 2000$ (Thermo Scientific, Langenselbold), X-tremeGENE ${ }^{\mathrm{TM}}$ HP DNA (Roche, Basel, Switzerland) and VCaP Cell Avalanche ${ }^{\circledR}$ (EZ Biosystems, Washington, DC, USA) were used in this study. Reagents were used according to the manufacturer's instructions. For Lipofectamine only half of the recommended reagent concentration was used to reduce cytotoxic effects.

\subsection{Functional analysis of eukaryotic cells}

\subsubsection{ApoTox-Glo ${ }^{\mathrm{TM}}$ Triplex Assay}

The ApoTox-Glo TM Triplex Assay by Promega was used to measure cell viability, cytotoxicity and activation of caspases on the same sample. This generates improved comparable data with smaller errors. The assay uses the two substrates glycyl-phenylalanylaminofluorocumarin (GF-AFC) and bis-alanylalanyl-phenylalanyl-rhodamine 110 (bis-AAF$\mathrm{R} 110$ ) to measure viability and cytotoxicity. GF-AFC is only cleaved by living cell protease in cells with an intact cell membrane; bis-AAF-R110 is cleaved by dying cells with a loosened membrane structure. The two cleaving products absorb light with different wavelength. The amount of cleaved product is proportional to the number of living and dead cells in the sample (see Fig. 2.3). 


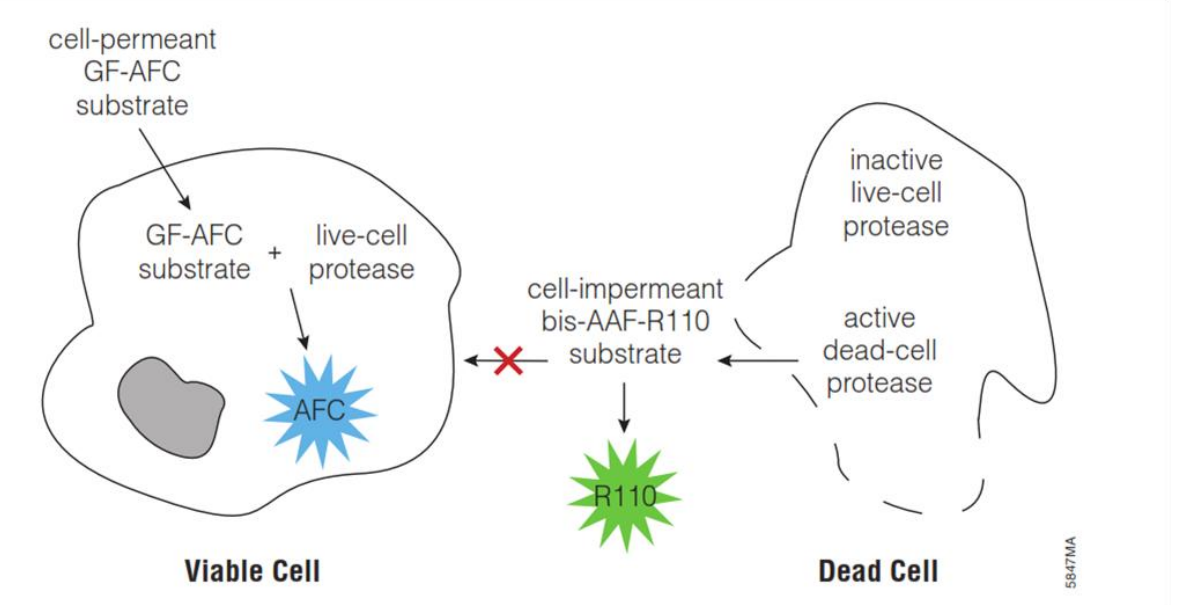

Figure 2.3: Schematic illustration of the measurement of living and dead cells with the ApoToxGlo $^{\text {TM }}$ Triplex Assay. The assay uses the two substrates GF-AFC and bis-AAF-R110. GF-AFC is only cleaved by living cells; bis-AAF-R110 is only cleaved by dying cells. The two cleaving products absorb light with different wavelength. The absorption is proportional to the number of cells. According to www.promega.com/ /media/Files/Resources/Protocols/Technical\%20Manuals/101/ApoTox-

Glo\%20Triplex\%20Assay\%20Protocol.pdf

The caspase activity is measured via luminescence. In a second step a luminogenic caspase 3/7 substrate is added which is cleaved by active caspases and release aminoluciferin. Aminoluciferin is metabolized by luciferase and this reaction emits light (see Fig. 2.4).

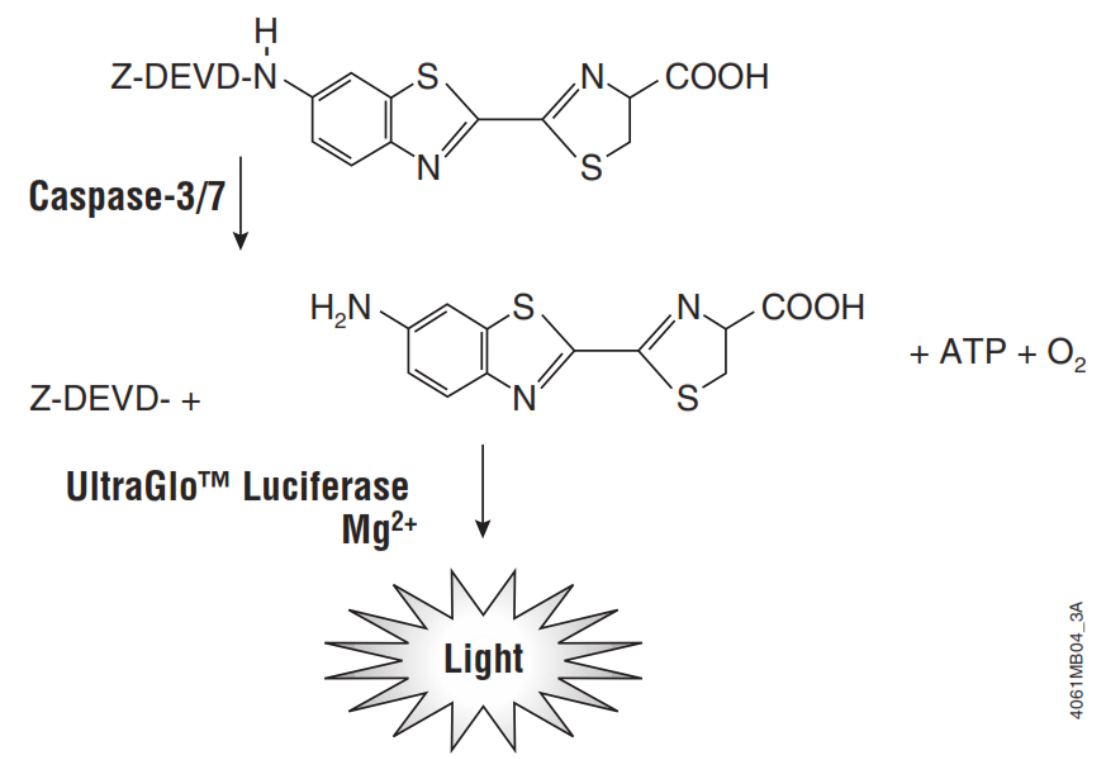

Figure 2.4: Schematic illustration of the caspase activity measurement with the ApoTox-G10 ${ }^{\mathrm{TM}}$ Triplex Assay. The caspases cleave the luminogenic caspase 3/7 substrate and therefore release aminoluciferin, which is cleaved by the luciferase and emits light. According to www.promega.com/ /media/Files/Resources/Protocols/Technical\%20Manuals/101/ApoTox-

Glo\%20Triplex\%20Assay\%20Protocol.pdf 
Here 15.000 VCaP cells, 3000 PC3 cells or 1500 2E or T244 cells were seeded in a 96-well, opaque-walled plate and treated with the respective agent. After $24 \mathrm{~h}, 48 \mathrm{~h}, 72 \mathrm{~h}$ or $144 \mathrm{~h}$ cell viability, cytotoxicity and caspase activation were measured with the Synergy Mx plate reader, following the manufacturer's instructions.

\subsubsection{Proliferation assay}

The cell proliferation rate was measured by the CellTiter $96 \AA$ AQueous Non-Radioactive Cell Proliferation Assay (Promega, Fitchburg, USA). The assay uses the reduction of tetrazoles (MTS) by mitochondria of living cells. In living cells, MTS in combination with the electron coupling reagent phenazine methosulfate (PMS) is reduced to formazan. The occurrence of formazan is measured by light absorbance maximum at $490 \mathrm{~nm}$. The intensity of the staining is relative to the number of proliferating cells.

Here, 3000 T244, 2E or BERKO/TRAMP 283 cells were plated in $100 \mu \mathrm{l}$ Adv. DMEM into a clear-bottom 96-well plate. Cells were allowed to settle for $2 \mathrm{~h}$. Proliferation was measured after $0 \mathrm{~h}, 24 \mathrm{~h}, 48 \mathrm{~h}$ and $72 \mathrm{~h}$. For measurement, $20 \mu \mathrm{l}$ MTS and $1 \mu \mathrm{l}$ PMS were added and cells were incubated for $2 \mathrm{~h}$ at $37^{\circ} \mathrm{C}$ and $5 \% \mathrm{CO}_{2}$. Absorption at $490 \mathrm{~nm}$ was measured with the Synergy Mx plate reader.

\subsubsection{Invasion assay}

Cell invasiveness was detected using the $\mathrm{BD}$ BioCoat ${ }^{\mathrm{TM}}$ Matrigel ${ }^{\mathrm{TM}}$ Invasion Chambers (BD Biosciences, Heidelberg). T244 and 2E cells were cultured in a matrigel-coated chamber. The matrigel coating serves as a barrier that can be passed by invasive growing cells.

Here, cells were pretreated with $20 \mathrm{mg} / \mathrm{ml}$ amygdalin for $24 \mathrm{~h}$. Untreated cells served as control. Then, 70.000 cells were seeded onto a matrigel chamber. To direct cell invasion a serum gradient was created between the medium within the chamber with serum reduced conditions (5\% FBS and $4 \%$ NuSerum) and medium in the bottom of the 24-well with $20 \%$ FBS and $16 \%$ NuSerum. Cells were allowed to grow for $24 \mathrm{~h}$ at $37^{\circ} \mathrm{C}$ and $5 \% \mathrm{CO}_{2}$. Those cells that grow through the matrigel were fixed with fixation buffer and stained with the DiffQuick staining kit (Dade Behring $\mathrm{GmbH}$, Marburg). Five photographs per membrane were randomly taken with the fluorescence microscope BX60 (Olympus, Hamburg, Germany) and the number of invaded cells treated with $20 \mathrm{mg} / \mathrm{ml}$ amygdalin was normalized to the number of invaded cells in the untreated control.

\subsubsection{Senescence-associated beta-galactosidase staining}

To detect senescent cells the senescence-associated $\beta$-galactosidase (SA $\beta$-gal) expression was stained. For cell culture analysis 2500 T244 or 2E cells were plated into 24 -well plates and incubated with medium containing $0 \mathrm{mg} / \mathrm{ml}, 5 \mathrm{mg} / \mathrm{ml}$ or $20 \mathrm{mg} / \mathrm{ml}$ amygdalin for $5 \mathrm{~d}$ or 
$7 \mathrm{~d}$. Cells were fixed in fixing solution for $10 \mathrm{~min}$ and afterwards washed twice with DPBS. The cells were incubated with staining solution at $37^{\circ} \mathrm{C}$ overnight and subsequently washed with DPBS and fixed again with $3.7 \%$ formaldehyde in DPBS for $10 \mathrm{~min}$. Photographs were taken on an inverted microscope (Olympus $\mathrm{GmbH}$, Hamburg) or Fluorescence microscope BX60 (Olympus GmbH, Hamburg).

\subsection{Dual-Luciferase Reporter Assay}

In order to measure the activity of transcription factors, a reporter system was used. Reporter systems utilize vectors carrying a transcription factor specific promoter region coupled to a reporter gene, e.g. luciferase. When the transcription factor is activated the expression of the marker gene is induced and can be detected, e.g. by Dual-Luciferase Reporter Assay (Promega, Fitchburg, USA).

Here, VCaP cells were transfected with reporter vectors for steroid receptor (MMTV-Luc)-, ER (vit-ERE-Luc)- and AR (rPb-Luc)-induced transcription and a PRL-Luc vector as reference for transfection efficacy. $24 \mathrm{~h}$ after transfection, cells were stimulated with the respective reagent and incubated for another $24 \mathrm{~h}$. The cells were lysed according to the manufacturer's instructions and luciferase activity was measured using the Synergy Mx plate reader.

\subsection{Immunohistochemistry}

\subsubsection{Immunofluorescence staining of eukaryotic cells}

Immunofluorescence staining of eukaryotic cells was used to visualize different features of adherent growing tumor cells. The cells were fixed on a slide and stained with fluorescent dyes.

The cells were allowed to grow on a 4 -well cell culture slide until they reached $70 \%$ confluence. Then, they were treated with the respective agent. The cells were fixed with fixing solution for $20 \mathrm{~min}$ and washed with DPBS for $10 \mathrm{~min}$. To stain intracellular proteins, $50 \mu \mathrm{l} 0.1 \%$ Triton X-100 (Fluka, Deisenhofen) was appended and incubated for $10 \mathrm{~min}$. Again, cells were washed with DPBS. To block all unspecific binding sites, blocking buffer (immunofluorescence) was added for $25 \mathrm{~min}$. Then, the primary antibody diluted in blocking buffer was supplied and incubated at $4^{\circ} \mathrm{C}$ overnight. The cells were washed twice in DPBS for 10 min. The secondary antibody was applied in blocking buffer and incubated at RT for $2 \mathrm{~h}$. The cells were washed again three times in DPBS and once with $\mathrm{H}_{2} \mathrm{O}$ for 10 min. $40 \mu \mathrm{l}$ Vectashield/DAPI was used to mount the slide and stain the nucleus. The signal was detected with the Confocal laser scanning microscope IX81 (Olympus GmbH, Hamburg) or Fluorescence microscope BX60. 
Table 2.19: Antibodies used for immunofluorescence. Listed are primary and secondary antibodies used for immunofluorescence analysis. The antibodies were diluted in $3 \%$ BSA in DPBS.

\begin{tabular}{l|c|c}
\hline Primary antibody & Primary antibody & Secondary antibody \\
\hline $\begin{array}{l}\text { a SV40 t/T antigen, monoclonal } \\
\text { antibody, mouse }\end{array}$ & $1: 500$ & $1: 500$ \\
a KI67, monoclonal antibody, & $1: 50$ & $1: 400$ \\
mouse & & $1: 800$ \\
$\begin{array}{l}\text { a } \mathrm{YH} 2 \mathrm{Ax}, \text { monoclonal antibody, } \\
\text { rabbit }\end{array}$ & $1: 1000$ &
\end{tabular}

\subsubsection{Immunohistochemical staining of paraffin embedded tissue sections}

Tissue analysis on formalin fixed, paraffin embedded tissue slides were performed using the EnVision Detection Systems Peroxidase/DAB, Rabbit/Mouse (Dako, Glostrup, Denmark). The app. $1 \mu \mathrm{m}$ thick tissue sections were treated with the hydrophobic clearing agent Xylene (Carl Roth $\mathrm{GmbH}$, Karlsruhe) for $20 \mathrm{~min}$ to remove the paraffin. Then, the sections were rehydrated in ethanol baths with progressively sinking concentrations, starting with $99 \%$, $90 \%, 85 \%, 80 \%$ and $70 \%$ ethanol for 2 min each. Slides were washed with $\mathrm{ddH}_{2} \mathrm{O}$ and boiled in citric buffer $\mathrm{pH} 6.0$ for $16 \mathrm{~min}$. The chamber was refilled every $3 \mathrm{~min}$ with $\mathrm{dd}_{2} \mathrm{O}$ to ensure all slides are covered with buffer. After boiling, the slides were slowly cooled down by rinsing with $d \mathrm{~d} \mathrm{H}_{2} \mathrm{O}$. Tissue specimens were circled with the ImmunoPen (Millipore, Billerica, USA). Then, specimens were covered with $3 \% \mathrm{H}_{2} \mathrm{O}_{2}$ for $20 \mathrm{~min}$ at $\mathrm{RT}$ in a humid atmosphere to block endogenous peroxidase activity. After rinsing the slides three times with DPBS, unspecific binding sites were blocked with $3 \% \mathrm{BSA} / 5 \% \mathrm{FBS}$ for $2 \mathrm{~h}$ at RT. Blocking buffer was removed and replaced by the primary antibody diluted in blocking buffer. Slides were incubated over night at $4^{\circ} \mathrm{C}$ in a humid atmosphere. The following day, the signal was visualized with the EnVision Detection Systems Peroxidase/DAB, Rabbit/Mouse according to the manufacturer's instructions under the Fluorescence microscope BX60 (Olympus, Hamburg). When the staining was sufficiently visible, the reaction was stopped by washing with DPBS. Cell and tissue structures were stained with hemalum (Carl Roth $\mathrm{GmbH}$, Karlsruhe). The slides were mounted with Gycergel (Dako, Glostrup, Denmark). Photographs were taken on the Fluorescence microscope BX60. 
Table 2.20: Antibodies used for IHC. Listed are primary and secondary antibodies used for IHC analysis. The antibodies were diluted in $3 \% \mathrm{BSA} / 5 \%$ FBS in DPBS.

\begin{tabular}{l|c|c}
\hline Primary antibody & Primary antibody & Secondary antibody \\
\hline $\begin{array}{l}\alpha \text { cleaved caspase 3, monoclonal } \\
\text { antibody, rabbit }\end{array}$ & $1: 500$ & $1: 500$ \\
& &
\end{tabular}

\subsection{Necrotic area calculation}

To analyze the necrotic area of PCa specimens, pictures were taken of hemalum and eosin stained tissue sections. Necrotic tissue is stained by eosin and therefore appeared pinkish. In contrast, intact tumor tissue is mostly composed of cells with large nuclei. Nuclei are stained by hemalum and thus appear bluish. The area of tumor cross sections and necrotic areas were measured by the cellSens program (Olympus, Hamburg). The percentage of necrotic area was calculated.

\subsection{In vivo experiments}

All in vivo studies were approved by the guidelines of the European Parliament (2010/63/EU) and the council for the protection of animals in science ( 14 TierSchVersV).

\subsubsection{Treatment of TRAMP-FVB mice with amygdalin}

For analyzing the effect of amygdalin on PCa growth in vivo TRAMP-FVB mice received i.p. amygdalin injection. Six weeks and 16 weeks old TRAMP-FVB mice were injected five times a week with amygdalin dissolved in $\mathrm{ddH}_{2} \mathrm{O}$. Different concentrations and degrees of purity were used. $\mathrm{ddH}_{2} \mathrm{O}$ was used as control. When mice developed palpable $\mathrm{PCa}>1 \mathrm{~cm}^{3}$ or health was impaired (criteria according to European and German protection of animals act) mice were sacrificed (Fig. 2.5). Organs of the urogenital tract such as prostate/PCa, seminal gland and testis and other organs such as liver, lung, kidney and spleen were cryoconservated for protein and RNA isolation and fixed in formalin for histopathological analysis. 


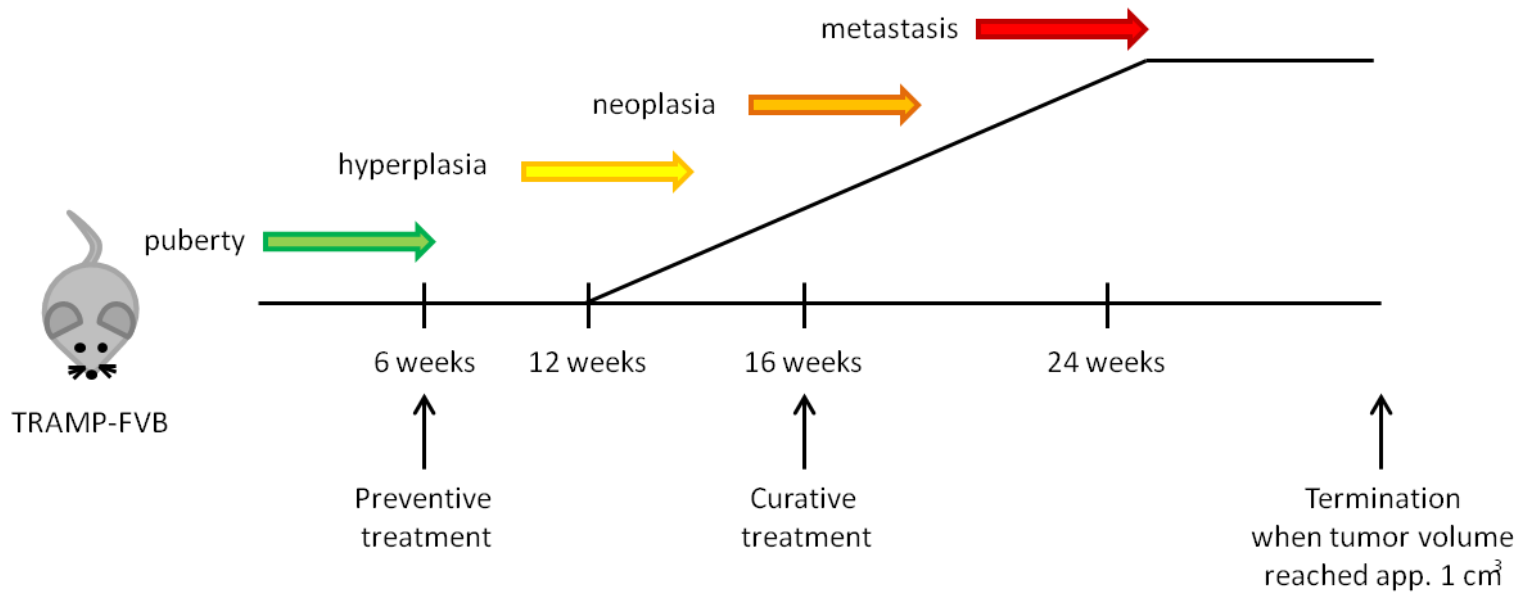

Figure 2.5: Amygdalin treatment scheme. TRAMP-FVB mice were treated with five intraperitoneal injections of amygdalin solution per week. Treatment was started six weeks or 16 weeks postnatal. The mice were sacrificed when PCa reached volumes $>1 \mathrm{~cm}^{3}$.

\subsubsection{Blood and serum extraction}

Immediately after amygdalin-treated mice were sacrificed, the thorax was opened and blood was obtained from the heart. For the isolation of EDTA blood the syringe was rinsed with $0.5 \mathrm{M}$ EDTA solution. Blood was kept at $-20^{\circ} \mathrm{C}$ or RT and analyses were performed as soon as possible. For serum isolation, blood was extracted and incubated $10 \mathrm{~min}$ on ice. Blood was centrifugated for $10 \mathrm{~min}$ at $1000 \mathrm{xg}$. After that, clear serum had separated from other blood components and could be transferred into a fresh tube and stored at $-80^{\circ} \mathrm{C}$.

\subsection{Cyanide measurement}

\subsubsection{Thiocyanate measurement}

[Scherer et al. 2017-under revision]

For thiocyanate $\left(\mathrm{SCN}^{-}\right)$measurement gas chromatography-coupled to mass spectrometry was used. The analysis was performed by Priv. Doz. Dr. rer. nat. Michael Müller of the Institute for Occupational, Social and Environmental Medicine of the University Medical Center, Göttingen. SCN ${ }^{-}$was derivated with 2,3,4,5,6-Pentafluorobenzylbromid (PFBBr) to 2,3,4,5,6-Pentafluorobenzylthiocyanat (PFBSCN) which could be detected by mass spectrometry (Fig. 2.6). 


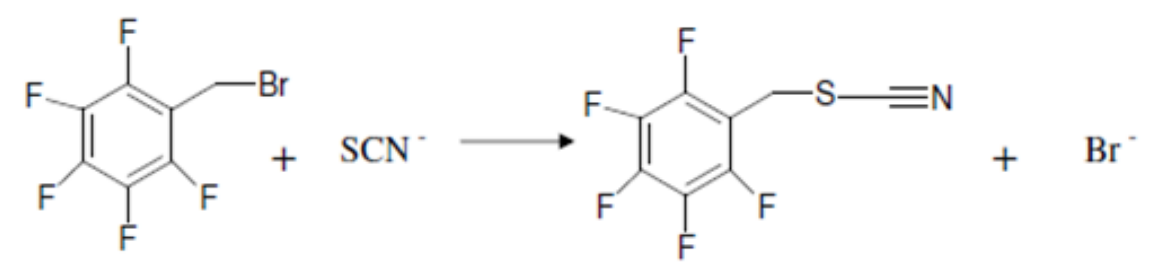

Figure 2.6: Chemical reaction of $\mathrm{PFBBr}$ to PFBSCN. To measure $\mathrm{SCN}^{-}$concentration via mass spectrometry, derivatisation to PFBBr was performed. Modified according to Scherer et al. 2017.

$100 \mu \mathrm{l}$ serum were diluted in $300 \mu \mathrm{l} \mathrm{H}_{2} \mathrm{O}$ and $25 \mu \mathrm{l}$ acetate buffer, $50 \mu \mathrm{l}$ IS-solution, $50 \mu \mathrm{l}$ $\mathrm{NaCl}$ solution, $25 \mu \mathrm{l}$ toluol (Promochem, Wesel) and $75 \mu \mathrm{l}$ derivatisation solution were appended and mixed for $30 \mathrm{~min} .2 \mathrm{ml}$ toluol were added and mixed for $5 \mathrm{~min}$. The solution was centrifuged for $10 \mathrm{~min}$ at RT at $2500 \times \mathrm{g}$ and $300 \mu \mathrm{l}$ of the toluol phase were transferred to a new vial. To remove dichlormethan residuals, the sample was constrained to $200 \mu \mathrm{l}$ using a vacuum centrifuge. For separation a RTX5 column $(25 \mathrm{~m} \times 0.25 \mathrm{~mm} \times 0.25 \mu \mathrm{m})$ (Restek, Bellefonte, USA) was used. Measurement was performed using the GC-MS (ThermoFisher Scientific, Dreieich).

\subsubsection{Cyanide measurement}

[Odoul et al. 1994]

Cyanide $\left(\mathrm{CN}^{-}\right)$concentration in EDTA blood was measured by gas chromatography coupled to mass spectrometry according to Odoul et al. The analysis was performed by Dr. rer. nat. Hans-Wolfgang Hoppe, head of the environmental toxicology of the medical center Bremen. $0.25 \mathrm{ml}$ blood were diluted in $2.5 \mathrm{ml} \mathrm{H} \mathrm{H}_{2} \mathrm{O}$ and $100 \mu \mathrm{l}$ phosphoric acid were added. The sample was appended into a $1 \%$ chloramine-T-solution (Sigma-Aldrich, Taufkirchen) filled vial and heated at $60^{\circ} \mathrm{C}$ for $30 \mathrm{~min}$. For separation a RTX5 column $(60 \mathrm{~m} \times 0.32 \mathrm{~mm} \times$ $1.5 \mu \mathrm{m}$ ) (Restek, Bellefonte, USA) was used. Samples were analyzed using the GC-MS QP2010plus (Shimadzu Scientific Instruments, Columbia, USA) and Headspace-Sampler Turbomatrix (PerkinEImer, Waltham, USA).

\subsection{Statistical analysis}

To confirm that the measured differences were statistically significant, the unpaired student's t-test was used. The P value was calculated by GraphPad. P value indicates the probability that the results are not different. A $P$ value smaller than * $-p<0.05$ means statistically significant; ${ }^{* *}-p<0.01$ means very significant; ${ }^{* * *}-p<0.0001$ means extremely significant.

Furthermore, statistic analyses were performed in cooperation with the TAL facility, University Medical Center Göttingen and Dr. Andreas Leha of the Head Core Facility for Medical Biometry and Statistical Bioinformatics, University Göttingen. 


\section{Results}

\subsection{Investigation of the role of estrogen receptors in prostate cancer}

\subsubsection{Analysis of the role of ER $\alpha$ and ER $\beta$ knockout in the TRAMP mouse model}

The function and molecular signaling of the two estrogen receptors (ERs) in prostate cancer (PCa) was studied using the established ERKO/TRAMP (ER a knockout/TRAMP) and BERKO/TRAMP (ER $\beta$ knockout/TRAMP) mouse models (Slusarz et al. 2012). In the present study, tumors from these mice should be used to generate primary PCa cell lines with an ER $\alpha$ or an ER $\beta$ knockout, respectively. These primary cell lines then should allow detailed analyses of the influence of ERs on PCa growth and the underlying molecular mechanisms.

\subsubsection{Survival times of ERKO, BERKO, TRAMP, ERKO/TRAMP and BERKO/TRAMP mice}

The survival of ERKO, BERKO, TRAMP, ERKO/TRAMP and BERKO/TRAMP mice was monitored. Mice were sacrificed when prostate tumor size reached volumes $>1 \mathrm{~cm}^{3}$ or burden of seminal gland cancer (SGCa) was apparent.

Either ERKO $(n=10)$ or BERKO $(n=9)$ mice did not develop prostate tumors, therefore, the experiment was terminated after approx. 400 days. On the one hand, BERKO/TRAMP $(n=12)$ and TRAMP $(n=23)$ mice showed similar median survival times ( $239 \pm 59$ days for BERKO/TRAMP and 225 \pm 96 days for TRAMP mice). On the other hand, ERKO/TRAMP ( $\mathrm{n}=$ 16) mice displayed a significantly reduced survival time of $160 \pm 45$ days compared to TRAMP mice(Fig. 3.1). For ERKO/TRAMP mice the experiment had to be terminated early due to the development of massive SGCa. About $30 \%$ of ERKO mice died for unknown reasons. 


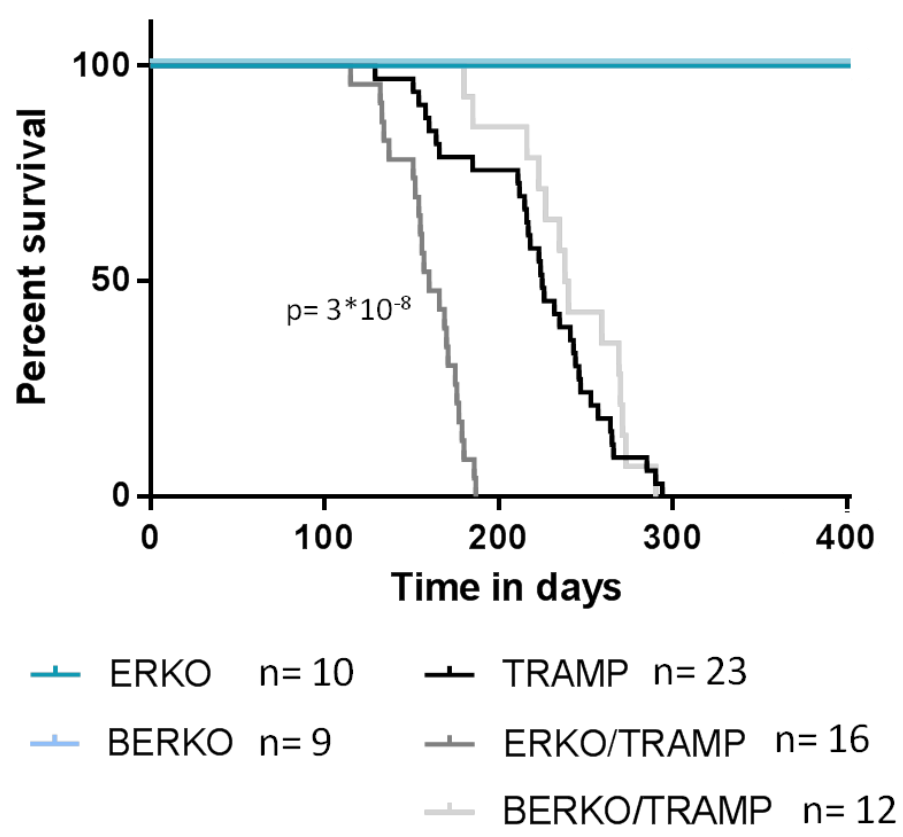

Figure 3.1: Survival of ERKO, BERKO, TRAMP, ERKO/TRAMP and BERKO/TRAMP mice. Depicted is the median survival of the different mouse strains in a Kaplan-Meier curve. ERKO $(n=10)$ and BERKO $(n=9)$ mice did not die of tumor burden during the course of the experiment. TRAMP $(n=23)$, ERKO/TRAMP $(n=16)$ and BERKO/TRAMP $(n=12)$ mice were sacrificed when tumors became burdensome. TRAMP and BERKO/TRAMP mice had a median survival of $225 \pm 96$ and $239 \pm 59$ days, respectively. ERKO/TRAMP mice showed a significantly reduced median survival of $160 \pm 45$ days. Significance was calculated using the student's t-test. ${ }^{*} p<0.05$, ${ }^{* *} p<0.01$, ${ }^{* \star *}$ $p<0.0001$ compared to TRAMP mice.

\subsubsection{Histopathological analysis of genotype-specific abnormalities in organ formation}

Tissue sections from different mouse organs were examined for abnormalities induced by the ER $\alpha$ knockout and ER $\beta$ knockout, respectively. After sacrificing the animals, the tumor, testis, seminal gland, lymph nodes, spleen, kidney, liver and lung were stored in formalin for histopathological analyses. Both the tumor stage and the formation of metastasis were evaluated together with Dr. med. Felix Bremmer, Department of Pathology, University Medical Center, Göttingen.

Pathological changes were not observed in any other organs than the urogenital tract of ERKO $(n=10)$, BERKO $(n=9)$ or TRAMP $(n=23)$ mice (Fig. 3.2 A). ERKO and BERKO mice did not develop PIN lesions in the prostate or tumors in other tissues. TRAMP and BERKO/TRAMP $(n=12)$ mice developed PIN lesions, PCa, metastatic PCa and SGCa. In these mice metastasis occurred predominately in lymph nodes and liver. ERKO/TRAMP $(n=$ 16) mice did not develop PCa, but PIN lesions were observed in the prostate of these mice as well as the occurrence of massive SGCa (Fig. 3.2 B). 

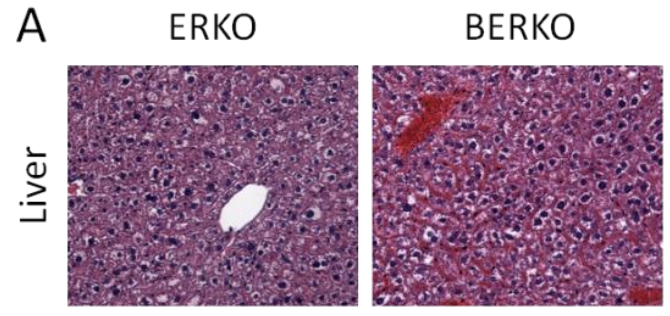

TRAMP
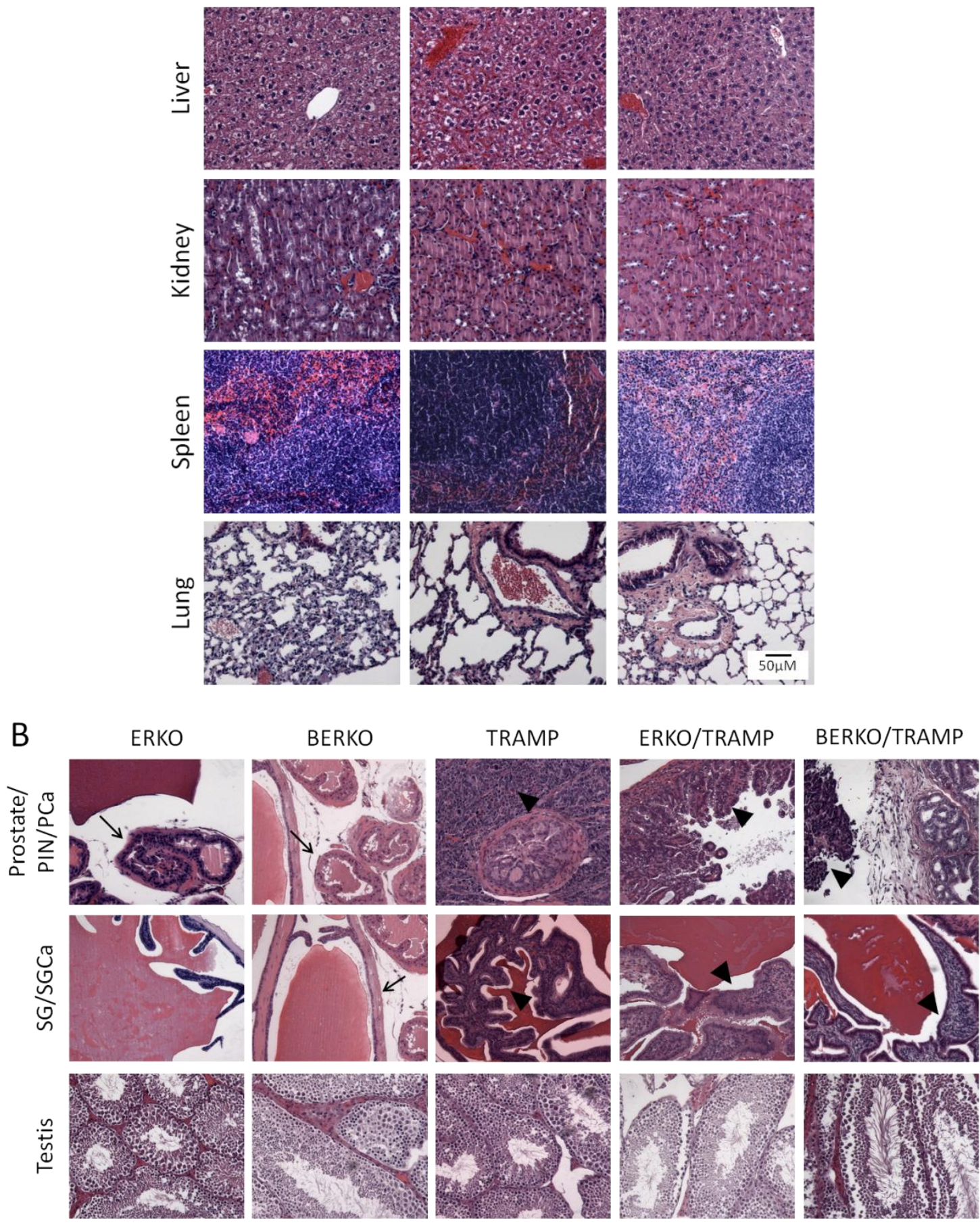

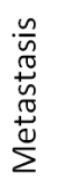
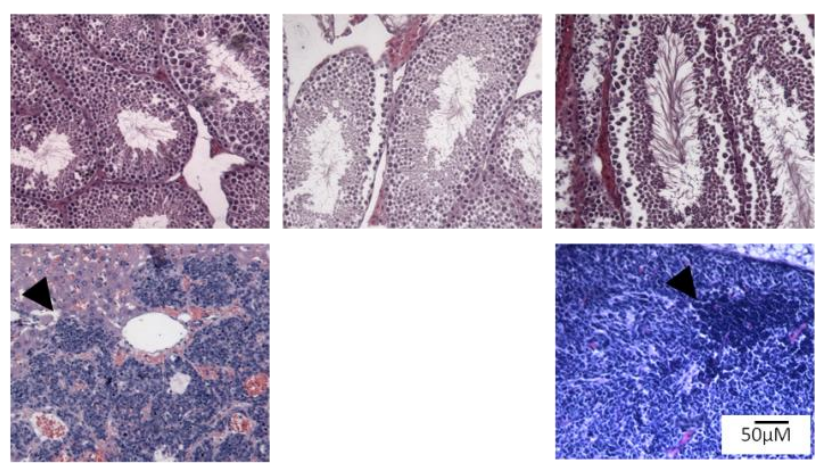

Figure 3.2: Hemalum and eosin stained tissue sections of ERKO, BERKO, TRAMP, ERKO/TRAMP and BERKO/TRAMP mice. Depicted are representative tissue sections of ERKO, BERKO, TRAMP, ERKO/TRAMP and BERKO/TRAMP mice. (A) No abnormalities in tissue histology were observed in organs such as liver, kidney, spleen or lung in ERKO, BERKO and TRAMP mice. (B) ERKO $(n=10)$ and BERKO $(n=9)$ mice did not show histological abnormalities in organs of the urogenital tract. TRAMP $(n=23)$, ERKO/TRAMP $(n=16)$ and BERKO/ TRAMP $(n=12)$ mice displayed 
development of PIN lesions and SGCa. TRAMP and BERKO/TRAMP mice developed metastatic PCa in lymph nodes and liver (Depicted for TRAMP mice is a liver metastasis, for BERKO/TRAMP mice a lymph node metastasis). Normal tissue (prostate and SG) is indicted by arrows, abnormal tissue (PCa, SGCa, metastasis) is indicated by arrowheads.

\subsubsection{Histopathologic analysis of the urogenital tract in TRAMP, ERKO/TRAMP and BERKO/TRAMP mice}

Histopathologic evaluation of the tumors and metastases was performed together with Dr. med. Felix Bremmer, Department of Pathology, University Medical Center, Göttingen. Tissue sections were categorized for the presence of $\mathrm{PIN}, \mathrm{PCa}$ and metastatic PCa. For the statistics, only the worst tumor stage for each mouse was included, e.g. if one mouse showed PIN lesions, PCa and metastatic PCa, this mouse was counted to have metastatic PCa. In contrast, the presence of SGCa was counted independently.

Approximately $56 \%$ of TRAMP mice $(n=16)$ tested developed PIN lesions, $17 \%$ PCa and $21 \%$ developed metastatic PCa. $65 \%$ of TRAMP mice developed SGCa. All ERKO/TRAMP mice analyzed $(n=16)$ had PIN lesions. $69 \%$ of ERKO/TRAMP mice developed SGCa. About $70 \%$ of BERKO/TRAMP mice evaluated $(n=12)$ showed PIN lesions, $15 \%$ PCa and $15 \%$ metastatic PCa (Fig. 3.3 A). $62 \%$ of BERKO/TRAMP mice showed SGCa (Fig. 3.3 B).

A

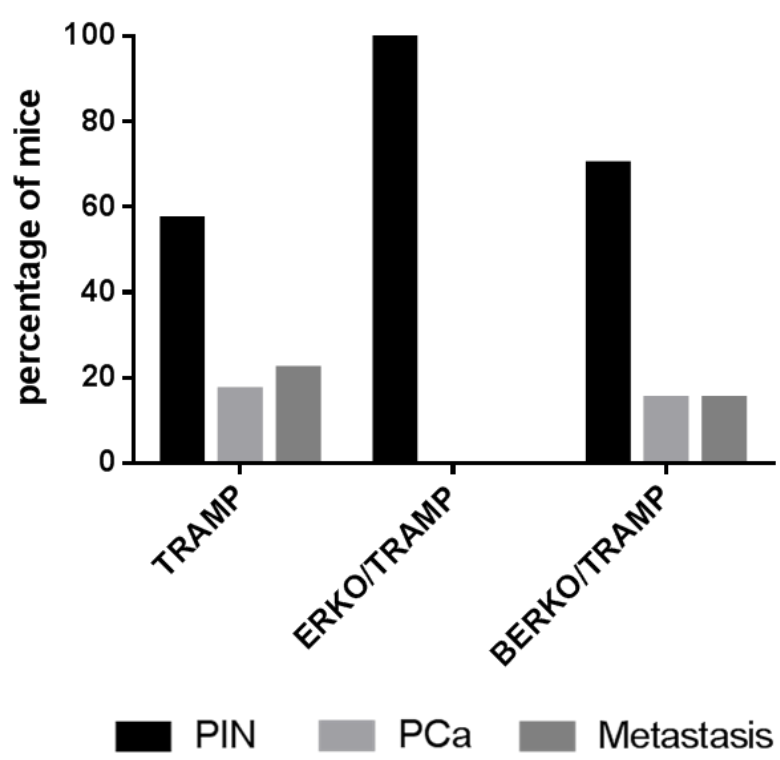

B

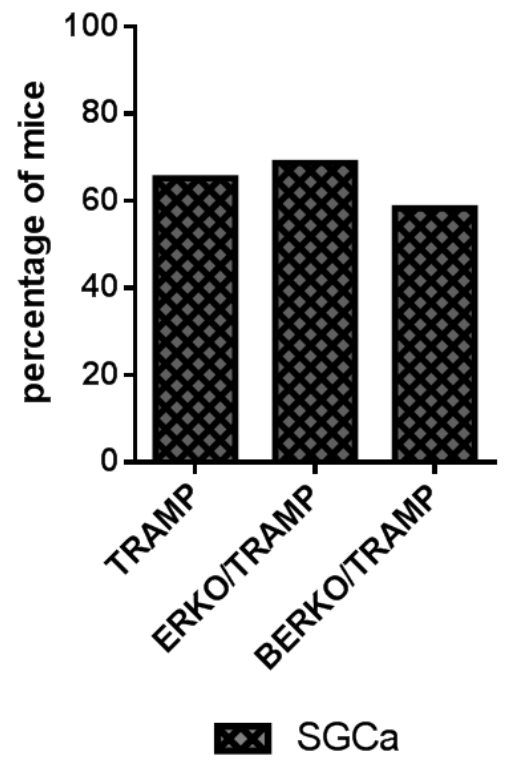

Figure 3.3: Histopathological analysis of prostates and seminal glands from TRAMP, ERKO/TRAMP and BERKO/TRAMP mice. Depicted is the relative occurrence of cancer entities in each study group in percent. (A) The majority of TRAMP and BERKO/TRAMP mice developed PIN lesions (56\% in TRAMP, $70 \%$ in BERKO/TRAMP). $17 \%$ of TRAMP mice showed PCa and $21 \%$ metastatic PCa. ERKO/TRAMP mice developed only PIN lesions. $15 \%$ of BERKO/TRAMP mice developed PCa and $15 \%$ metastatic PCa. (B) SGCa occurred in all groups to approx. equal percentage. TRAMP $n=23$, ERKO/TRAMP $n=16$, BERKO/TRAMP $n=12$ 


\subsubsection{Establishment of PCa cell lines from TRAMP and BERKO/TRAMP mice}

The role of ER signaling in PCa should be analyzed in primary PCa cell lines. ERKO/TRAMP mice did not develop PCa hence a cell line with this genotype could not be obtained. Therefore, cell lines from PCa tumors of TRAMP and BERKO/TRAMP mice were generated. To establish primary PCa cell culture, the tumors were harvested, cells were solved from tissue and cultured at $37^{\circ} \mathrm{C}$ and $5 \% \mathrm{CO}_{2}$ (see 2.19.1). The TRAMP cell line T244 and the BERKO/TRAMP cell line BERKO/TRAMP 283 were generated according to this protocol. To confirm tumor cell origin and exclude fibroblast contamination, a SV40 large T antigen staining was performed (see 2.22.1). As a positive control, murine primary PCa $2 \mathrm{E}$ cells previously generated from a TRAMP mouse PCa by Dr. Sandra von Hardenberg (von Hardenberg, 2010) and characterized by Dr. Daria Witt were used (Witt, 2013).

Both PCa cell lines T244 and BERKO/TRAMP 283 expressed the SV40 large T antigen in $100 \%$ of cells and fibroblast contamination could not be observed (Fig. 3.4).

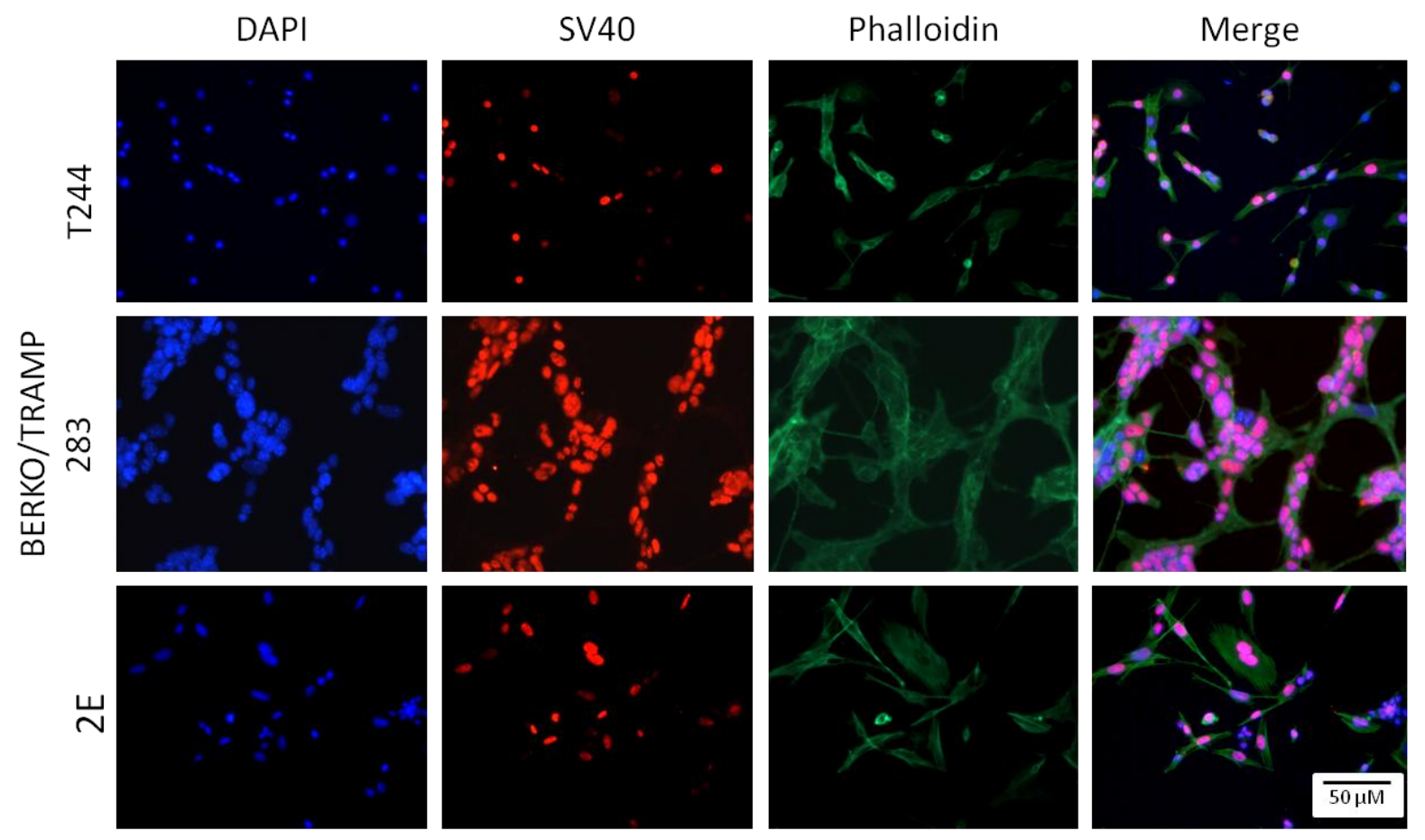

Figure 3.4: SV40 large T antigen expression in T244, BERKO/TRAMP 283 and 2E cells. Cells were plated on 4-well slides and SV40 large T expression (red), nuclei (DAPI) and cytoskeleton (green) were stained. SV40 large T antigen is expressed in newly established primary cell lines derived from TRAMP (T244) and BERKO/TRAMP (BERKO/TRAMP 283) mice, whereas 2E cells served as positive control.

\subsubsection{Confirmation of the Esr2 knockout genotype in BERKO/TRAMP 283 cells}

The genotype of each mouse used in this study was identified by genotyping PCR (see 2.17.1). In order to confirm the homozygous knockout of ER $\beta$ gene (Esr2) in the BERKO/TRAMP 283 cell line the genotyping PCR was repeated with genomic DNA isolated 
from these cells (see 2.13.2). The primer pairs ESR2-MNeo25, ESR2-MB1-2sp and ESR2MC1wTnew were used for the PCR (Krege et al. 1998). BERKO/TRAMP 283 cells showed only a PCR product of the BERKO alleles with $500 \mathrm{bp}$ in size, i.e. a homozygous ER $\beta$ knockout could be verified (Fig. 3.5).

A

Wild type Esr2

PCR product $844 \mathrm{bp}$

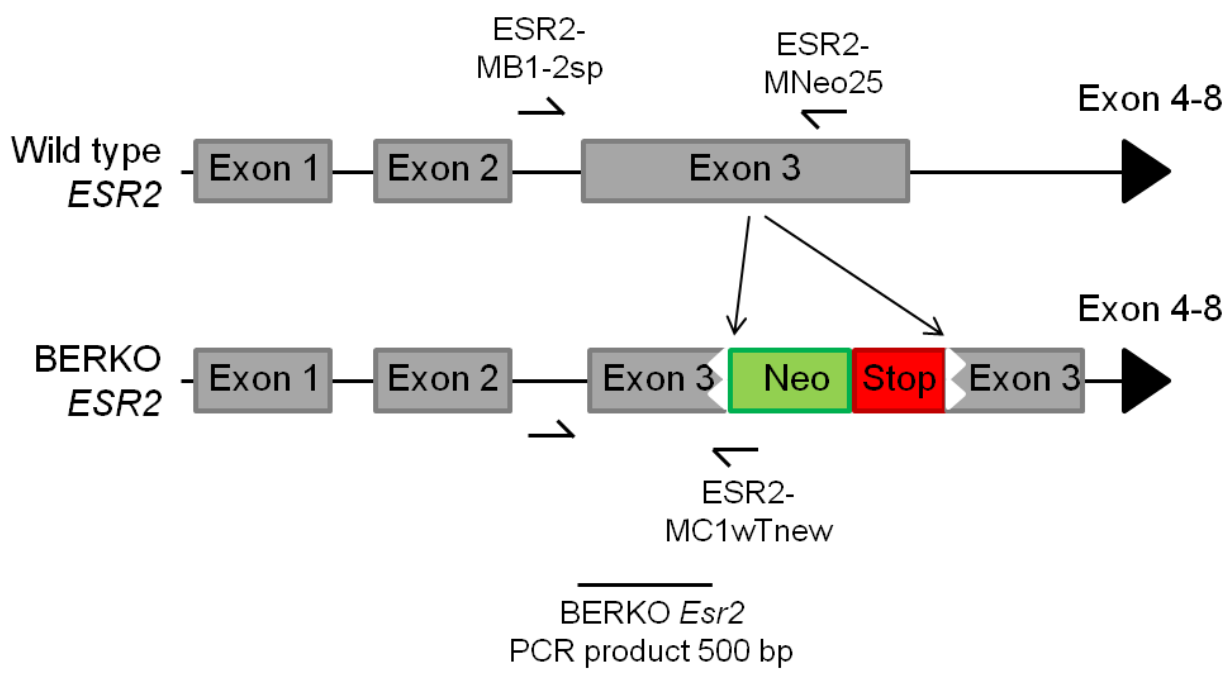

B

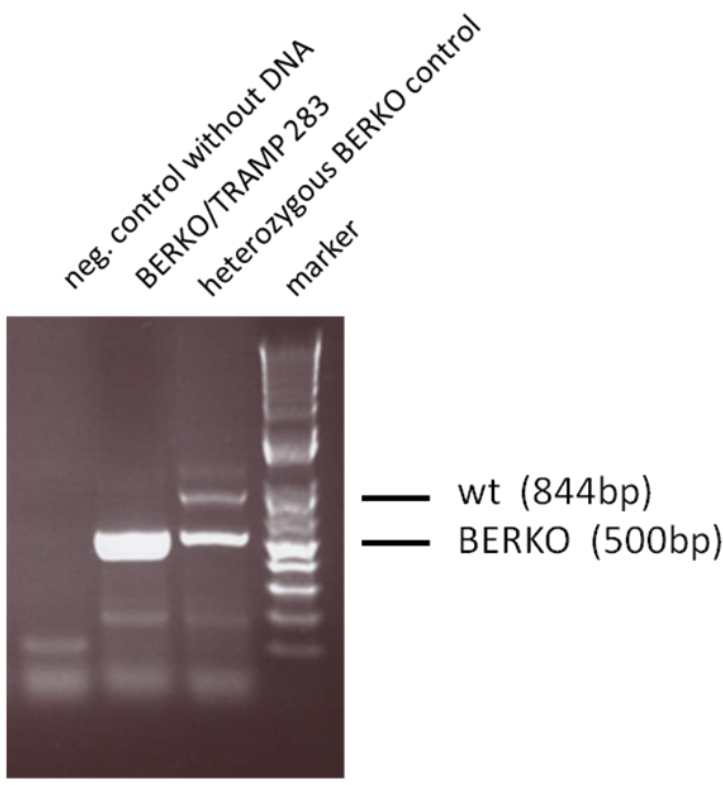

Figure 3.5: Confirmation of the BERKO genotype in the BERKO/TRAMP 283 cell line. (A) Schematic illustration of the BERKO Esr2 gene construct. A neomycin cassette with a terminating stop codon is integrated into exon 3 of the Esr2 gene in BERKO mice. To confirm the genotype of the Esr2 gene knockout the primer pairs ESR2-MNeo25 and ESR2-MB1-2sp and ESR2-MB1-2sp and ESR2MC1wTnew (indicated by arrows) were used for the amplification. (B) Genotyping PCR confirmed that BERKO/TRAMP 283 cells showed only a PCR product of homozygous BERKO alleles with $500 \mathrm{bp}$ in length. As a further control genomic DNA derived from a tail biopsy of a heterozygous BERKO mouse was used for PCR. As expected, PCR products of $844 \mathrm{bp}$ and $500 \mathrm{bp}$ in size were obtained for both the wild type (wt) ER $\beta$ allele and the BERKO allele. Marker= $1 \mathrm{~Kb}$ Plus DNA Ladder. 


\subsubsection{Expression of ER $\beta$ in BERKO/TRAMP 283 and T244 cells}

To validate the knockout of ER $\beta$ in BERKO/TRAMP 283 cells at the protein level a western blot analysis was performed. Whole proteins of BERKO/TRAMP 283 and T244 cells were immunoblotted and incubated with an ER $\beta$-specific antibody.

Surprisingly, using the ER $\beta$-specific antibody \#5513 from the company Cell signaling both BERKO/TRAMP 283 and T244 cells showed strong expression of ER $\beta$ (Fig. 3.6).

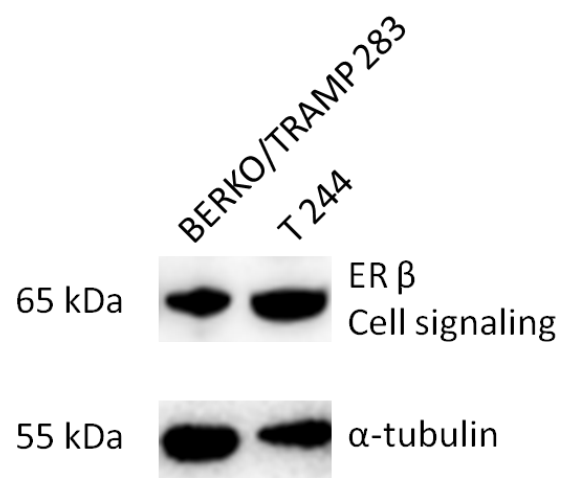

Figure 3.6: ER $\beta$ expression in BERKO/TRAMP 283 and T244 primary cell lines. Proteins of BERKO/TRAMP 283 and T244 cells were isolated and immunoblotted with the ER $\beta$-specific antibody \#5513 from Cell signaling. Both cell lines showed strong expression of ER $\beta$ (upper panel). A representative western blot of two independent experiments which were performed in duplicates is shown. $\alpha$-tubulin was used as loading control (lower panel).

To exclude the possibility of either a contamination in the BERKO/TRAMP 283 cell line or an unspecific reaction of the antibody, further analyses were performed. Proteins derived from fresh frozen tissue from kidney of BERKO/TRAMP mice, liver, testis and prostate of TRAMPFVB mice, human VCaP cells, primary murine PCa cell line T244 and mouse brain as positive control were immunoblotted with the ER $\beta$-specific antibody ab3577 (Abcam). Different tissues and cell lines should elucidate if ER $\beta$ expression differs between tissues and if ER $\beta$ antibody detects signals of murine and human origin. Again, strong expression of ER $\beta$ was detected in ER $\beta$ wild type as well as in homozygous BERKO tissues. The proteins of T244 cells, BERKO/TRAMP kidney and TRAMP-FVB liver showed next to the predicted band at $65 \mathrm{kDa}$ an additional signal at $55 \mathrm{kDa}$. To confirm the specificity of the two signals, the ER $\beta$ blocking peptide ab3564 (Abcam) was used. The signal of both bands was diminished nearly completely indicating that the antibody is specific for ER $\beta$ and that there are presumably different ER $\beta$ isoforms present (Fig. 3.7) 


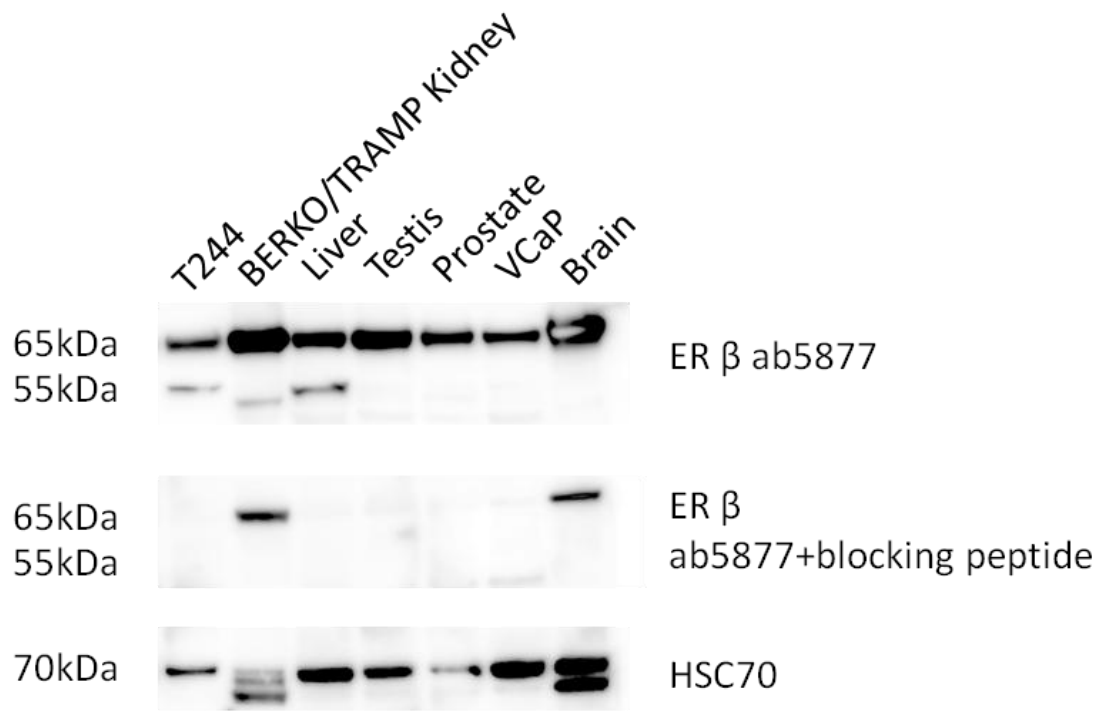

Figure 3.7: Confirmation of ER $\beta$ antibody specificity. Proteins of T244 cells and kidney of BERKO/TRAMP mice, liver, testis and prostate of TRAMP-FVB mice, human VCaP cells and mouse brain as positive control were isolated and immunoblotted with the ER $\beta$-specific antibody ab5877 (Abcam). All tissues showed expression of ER $\beta$, with two bands detected on $55 \mathrm{kDa}$ and $65 \mathrm{kDa}$ (upper panel). The experiment was repeated with the addition of an ER $\beta$-specific blocking peptide (middle panel). ER $\beta$ signal for both bands was diminished for most samples, except kidney from BERKO/TRAMP mice and mouse brain positive control. HSC70 was used as loading control (lower panel).

\subsubsection{Sequencing of exon 3 of the Esr2 gene to confirm the insertion of a stop codon}

The knockout of ER $\beta$ was generated by the insertion of a neomycin cassette and a stop codon in exon 3 of the mouse Esr2 gene, thus terminating mRNA translation (Krege et al. 1998, Fig. 3.8). To confirm the correct integration of the stop codon in the mouse Esr2 gene, genomic DNA was isolated from BERKO/TRAMP 283 cells and PCR with the specific primers Neo-fw1 and Neo-rev1 located in the neomycin cassette and in exon 3 of the mouse Esr2 gene was performed. Subsequent bidirectional sequence analysis confirmed the correct orientation of the construct in exon 3 of the mouse Esr2 gene and the ER $\beta$ knockout genotype in BERKO/TRAMP 283 cells. Furthermore, mutations or sequence aberrations were not identified. 


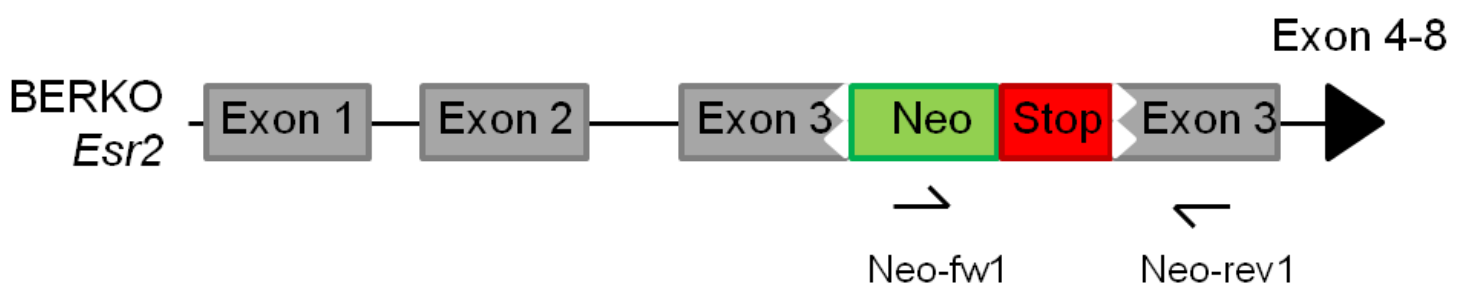

Figure 3.8: Schematic illustration of the BERKO Esr2 gene. A neomycin cassette with a terminating stop codon is integrated into the Esr2 gene in BERKO mice. To confirm the genotype and functionality of the Esr2 knockout a sequence reaction was performed, validating the neomycin cassette and the stop codon. Used primers (Neo-fw1 and Neo-rev1) are indicated by arrows. No sequence aberrations were found.

\subsubsection{Analysis of the proliferation rate of T244 and BERKO/TRAMP 283 cells}

To analyze if $\mathrm{PCa}$ cells derived from a BERKO/TRAMP mouse tumor differ in their proliferation compared to PCa cell lines generated from TRAMP mouse tumors, proliferation of T244 (TRAMP), 2E (TRAMP) and BERKO/TRAMP 283 cells was measured using the MTS-based proliferation assay (see 2.20.2). Proliferation was measured after $0 \mathrm{~h}, 24 \mathrm{~h}, 48 \mathrm{~h}$ and $72 \mathrm{~h}$.

T244 cells showed a significant higher proliferation rate as $2 \mathrm{E}$ cells $(41 \%$ after $72 \mathrm{~h}$ ) and BERKO/TRAMP 283 (38\% after $72 \mathrm{~h}$ ) cells. No difference in proliferation rate between $2 \mathrm{E}$ and BERKO/TRAMP 283 cells was observed (Fig. 3.9).

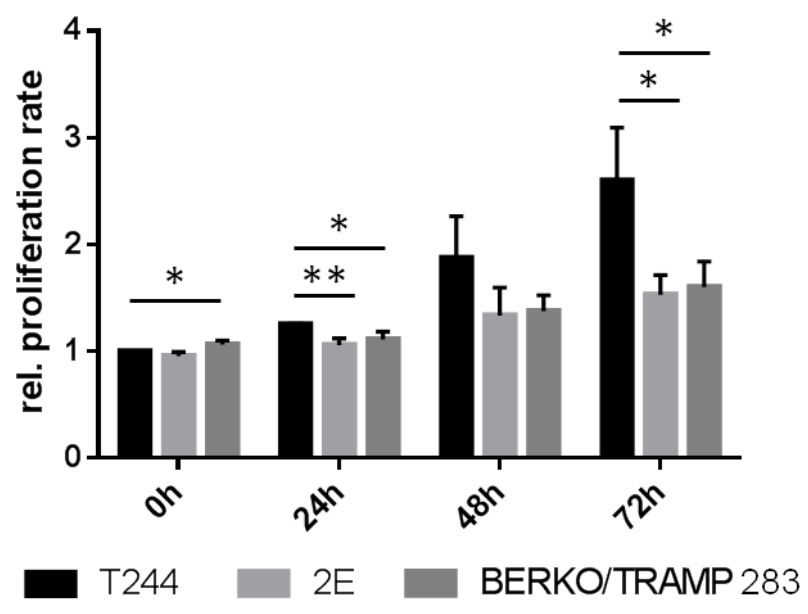

Figure 3.9: Proliferation of murine, primary PCa cells T244, 2E and BERKO/TRAMP 283. Proliferation rate of T244, 2E and BERKO/TRAMP 283 cells was measured using a MTS-based proliferation assay. T244 cells showed a significant higher proliferation rate compared to $2 \mathrm{E}$ and BERKO/TRAMP 283 cells. No difference in the proliferation rate between $2 \mathrm{E}$ and BERKO/TRAMP 283 cells was observed. The data represent the mean $\pm s . d$ of three independent experiments which were performed in triplicates. Significance was calculated using the student's t-test. ${ }^{*} p<0.05,{ }^{* *} p<0.01$, ${ }^{* * *} p<0.0001$ compared to T244 cells. 


\subsubsection{ER $\beta$ activation in PCa cell lines}

Next to the knockout of ER $\beta$ expression in mouse PCa cell lines, the activation of ER $\beta$ could give information about the role of ER $\beta$ in PCa. Here, we used a human PCa cell system originally generated by PD Dr. Paul Thelen, Department of Urology, University Medical Center, Göttingen to investigate the effects of ER $\beta$ activation in different PCa stages. This cell system was composed of three VCaP cell variants. Therefore, VCaP cells were treated seven month with $1 \mathrm{nM}$ testosterone and thereby reverted back into an androgen-dependent cell line. At the opposite, VCaP cells were treated with the agent abiraterone for three weeks until they became resistant to this androgen ablation therapy. Thus, these three VCaP cell variants originated from the same cell line mimic early (VCaP rev.), advanced (VCaP) and treatment resistant (VCaP AA) PCa stages (Fig. 3.10). Therefore, the VCaP cell system allows insights into differentially reactions to treatment during PCa progression.

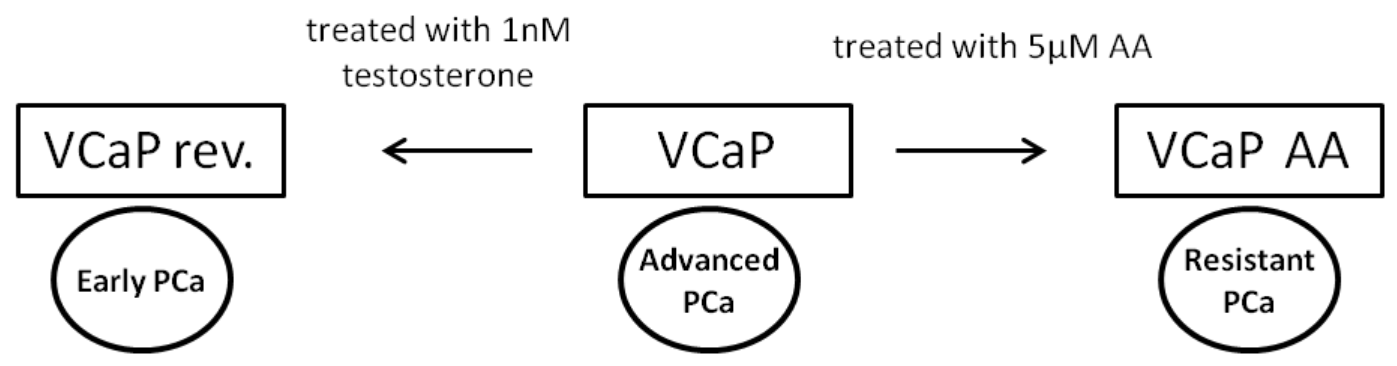

Figure 3.10: Schematic illustration of PCa stages represented by VCaP cell variants. VCaP rev. cells were cultured with $1 \mathrm{nM}$ testosterone over seven months, while VCaP AA cells were treated with $5 \mu \mathrm{M}$ abiraterone acetate for three weeks. The three VCaP cells lines represent three different levels of androgen deprivation therapy (ADT), VCaP rev: early PCa, before ADT therapy; VCaP: advanced PCa under $1^{\text {st }}$ line therapy, overexpression of AR as adaptation to ADT; VCaP AA: resistant PCa under maximal ADT.

\subsubsection{Identification of differentially expressed genes in VCaP rev., VCaP and VCaP AA cells}

First, it was investigated if the different treatments generate a VCaP cell system that mimics the different stages of PCa. Therefore, the AR status and the expression of the AR target gene PSA in the three VCaP cell variants was examined (Fig. 3.11). RNA or protein was isolated from untreated VCaP rev., VCaP and VCaP AA cells. Quantitative RT-PCR was performed to analyze mRNA expression of $A R$ and PSA. AR-specific, ER $\beta$-specific and PSA-specific antibodies were used to detect protein expression by western blot analysis (see 2.17.4, 2.18).

VCaP cells showed a high expression of $A R$ and PSA mRNA as well as AR protein. In comparison to $\mathrm{VCaP}$ cells, $\mathrm{VCaP}$ rev. cells showed a significantly reduced mRNA expression of $A R$ (reduced by $88 \%$ ) and PSA (reduced by $87 \%$ ) (Fig. 3.11 A). Also protein expression 
of $\mathrm{AR}$ and PSA were reduced in VCaP rev. cells compared to VCaP cells. In VCaP AA cells the AR protein expression was even increased compared to VCaP cells and also AR splice variants (ARVs) were detected. Protein expression of PSA was increased in VCaP AA cells compared to VCaP cells. In VCaP rev. and VCaP AA cells the ER $\beta$ expression was lower than in VCaP cells (Fig. 3.11 B).

A

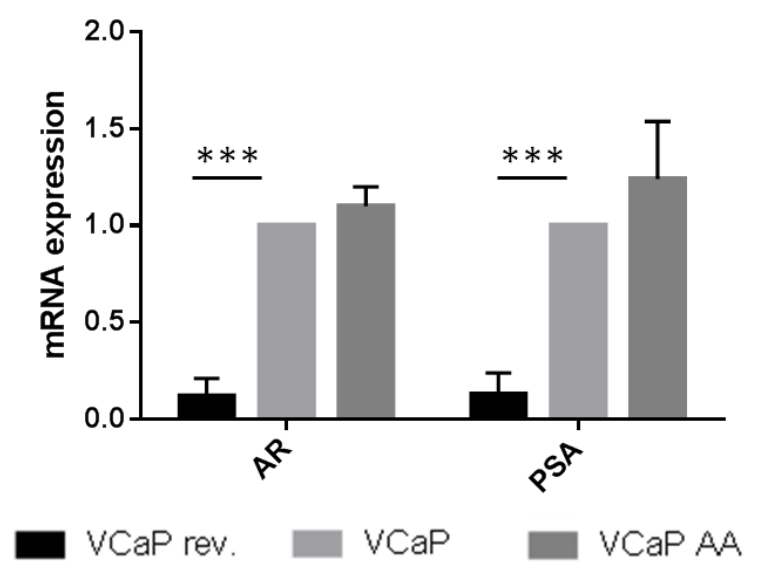

B
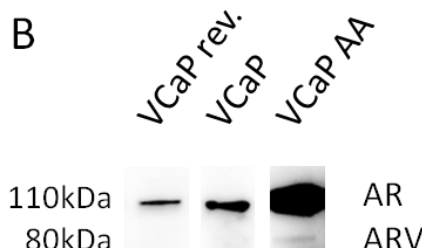

$65 \mathrm{kDa}-\boldsymbol{E}-\mathrm{ER} \beta$

$29 \mathrm{kDa} \quad \ldots$ PSA

$55 k D a \longrightarrow \alpha$-tubulin

Figure 3.11: Expression of PCa-relevant genes in VCaP cell variants. (A) mRNA expression of $A R$ and PSA were measured by quantitative RT-PCR in all VCaP cell lines. $A R$ and PSA expression in VCaP rev. cells was significantly lower compared to VCaP cells. $A R$ and PSA mRNA expression in VCaP AA cells was not significantly higher than in VCaP cells. Data represent the mean $\pm \mathrm{s}$.d of three independent experiments performed in duplicates. Significance was calculated using the student's ttest. ${ }^{*} p<0.05,{ }^{* *} p<0.01,{ }^{* * *} p<0.0001$ compared to VCaP cells. (B) Protein expression of AR, ER $\beta$ and PSA was analyzed by western blot in all VCaP cell variants. Total protein was extracted and immunoblots were probed with AR-specific (upper panel), ER $\beta$-specific (second panel) and PSAspecific (third panel) antibodies, respectively. In VCaP rev. cells AR and PSA expression was remarkably lower than in VCaP cells. In VCaP AA cells the AR and PSA expression level was increased compared to VCaP cells. In VCaP rev. as well as in VCaP AA cells the ER $\beta$ expression was lower than in VCaP cells. A representative western blot of two independent experiments performed in duplicates is shown. $\alpha$-tubulin was used as a loading control (lower panel).

\subsubsection{Analysis of the effect of $8 \beta-V E 2$ treatment in VCaP cell variants}

To activate the ER $\beta$ in the VCaP cell variants, cells were treated with the ER $\beta$-specific ligand $8 \beta$-VE2 (8-vinylestra-1,3,5 (10)-triene-3,17 $\beta$-diol). First, a pretrial was performed to evaluate appropriate concentrations of $8 \beta-\mathrm{VE} 2$ for VCaP cell treatment. Therefore, VCaP cells were treated with DMSO, $5 \mu \mathrm{M}, 12.5 \mu \mathrm{M}, 25 \mu \mathrm{M}$ and $50 \mu \mathrm{M} 8 \beta$-VE2 for $24 \mathrm{~h}, 48 \mathrm{~h}$ and $72 \mathrm{~h}$, respectively. Subsequently, both VCaP cell survival and apoptosis induction were measured (see 2.20.1). 
The $8 \beta$-VE2 concentrations at $5 \mu \mathrm{M}$ and $25 \mu \mathrm{M}$ were chosen for further experiments, since lower $8 \beta$-VE2 concentrations failed to induce effects and higher $8 \beta$-VE2 concentrations induced rather unspecific VCaP cell degradation (Fig. 3.12).

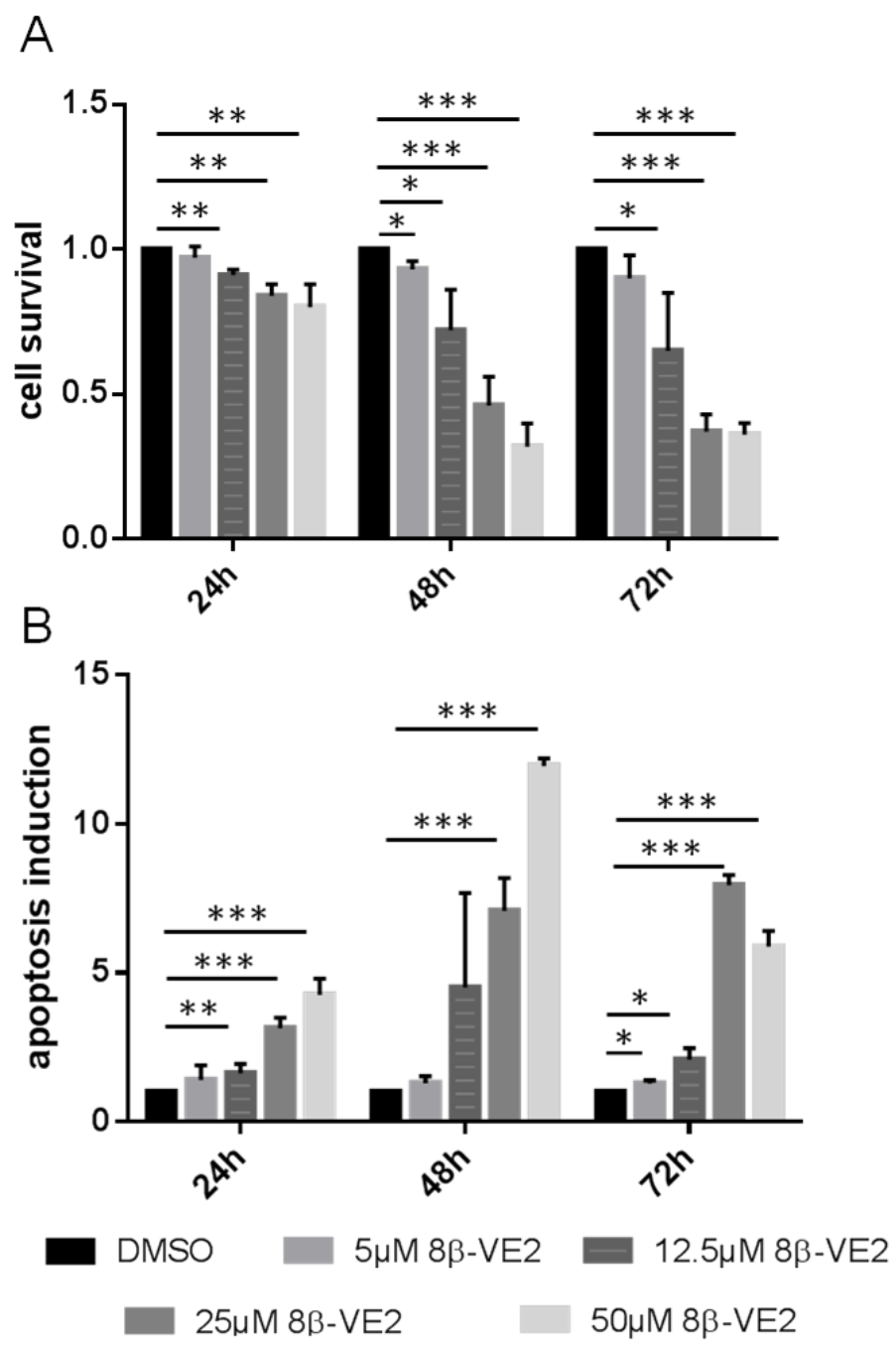

Figure 3.12: Efficacy evaluation of ER $\beta$-specific agonist $8 \beta$-VE2 in VCaP cells. VCaP cells were treated with $5 \mu \mathrm{M}, 12.5 \mu \mathrm{M}, 25 \mu \mathrm{M}, 50 \mu \mathrm{M} 8 \beta$-VE2 or DMSO as control and (A) cell survival and (B) apoptosis were measured after $24 \mathrm{~h}, 48 \mathrm{~h}$, and $72 \mathrm{~h}$, respectively. Concentrations of $8 \beta-V E 2$ at $5 \mu \mathrm{M}$ and $25 \mu \mathrm{M} 8 \beta$-VE2 were used for further experiments. Data represent the mean \pm s.d of three independent experiments performed in duplicates. Significance was calculated using the student's ttest. ${ }^{\star} p<0.05,{ }^{* *} p<0.01,{ }^{* * *} p<0.0001$ compared to VCaP cells.

To analyze the effect of $8 \beta$-VE2 treatment on VCaP cell variants, VCaP rev., VCaP and VCaP AA cells were treated with DMSO, $5 \mu \mathrm{M}$ and $25 \mu \mathrm{M} 8 \beta$-VE2 for $24 \mathrm{~h}, 48 \mathrm{~h}$ and $72 \mathrm{~h}$, respectively. Next, cell survival and apoptosis induction were measured in all three cell lines. Cell survival reduction and induced apoptosis were observed in all three VCaP cell variants upon treatment with $25 \mu \mathrm{M} 8 \beta$-VE2. Strongest cell survival reduction was detected after $72 \mathrm{~h}$ treatment, i.e. for $\mathrm{VCaP}$ rev. cells $48 \%$, for $\mathrm{VCaP}$ cells $57 \%$ and for $\mathrm{VCaP}$ AA cells $53 \%$ reduced survival (Fig. 3.13 A, C, E). Apoptosis induction was consistently strong after $24 \mathrm{~h}$, 
$48 \mathrm{~h}$ and $72 \mathrm{~h}$ in VCaP rev. cells (2.7-fold compared to control cells). VCaP cells showed strongest apoptosis induction after $72 \mathrm{~h}$ (5.3-fold compared to control cells). In VCaP AA cells early apoptosis after $24 \mathrm{~h}$ was observed (5.1-fold compared to control cells) (Fig. 3.13 $B, D, F)$. 
A

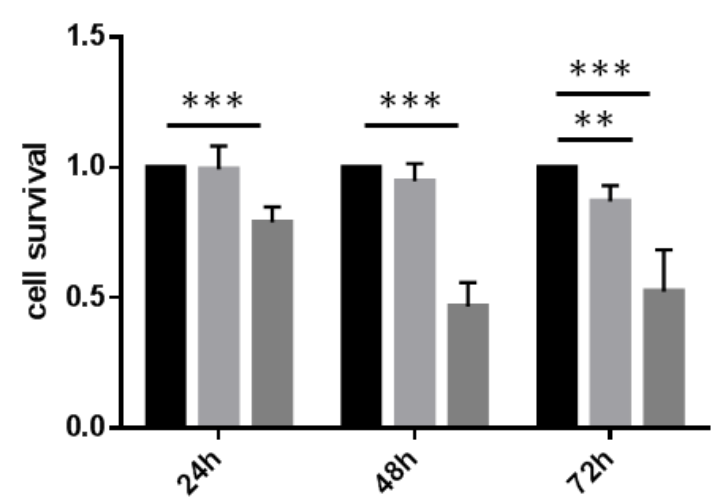

C

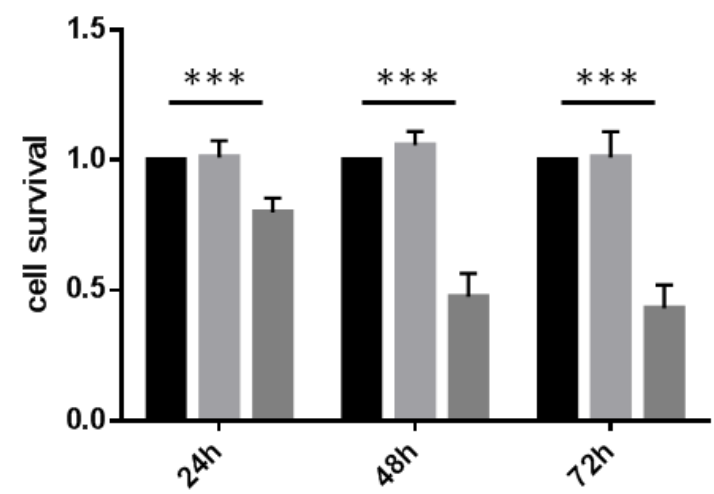

E

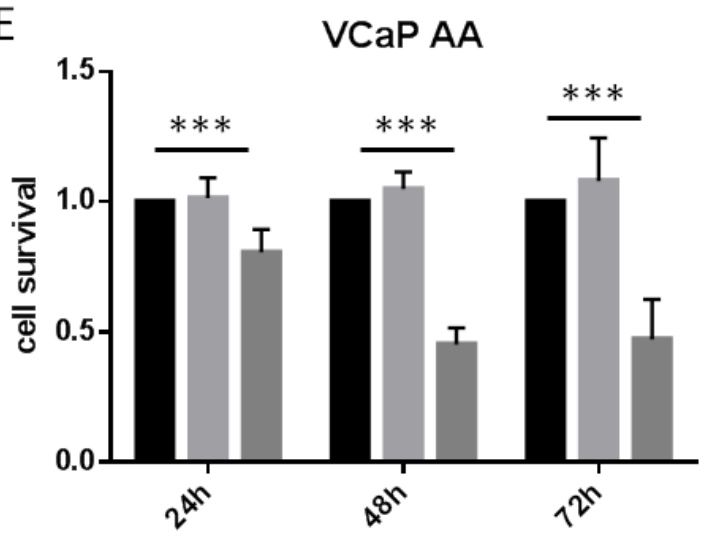

B

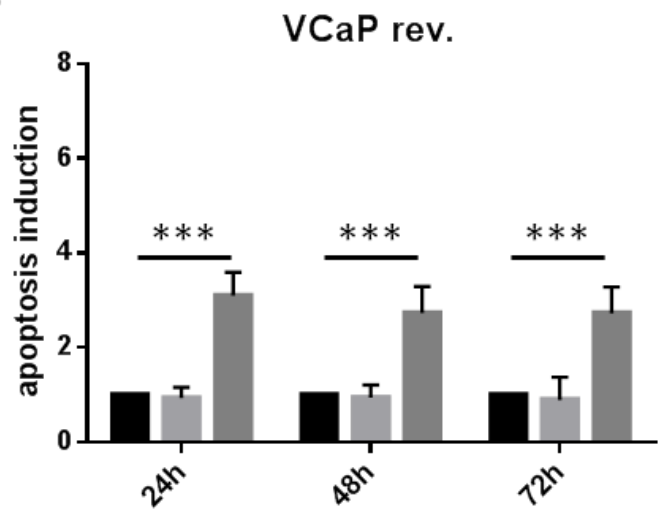

D

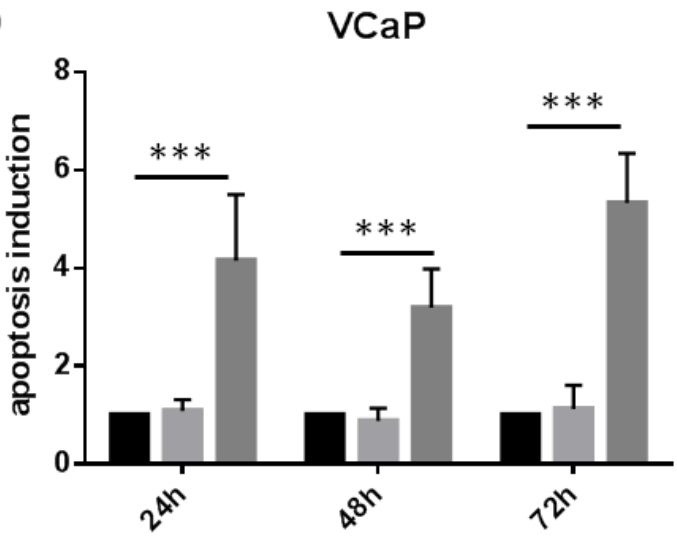

F

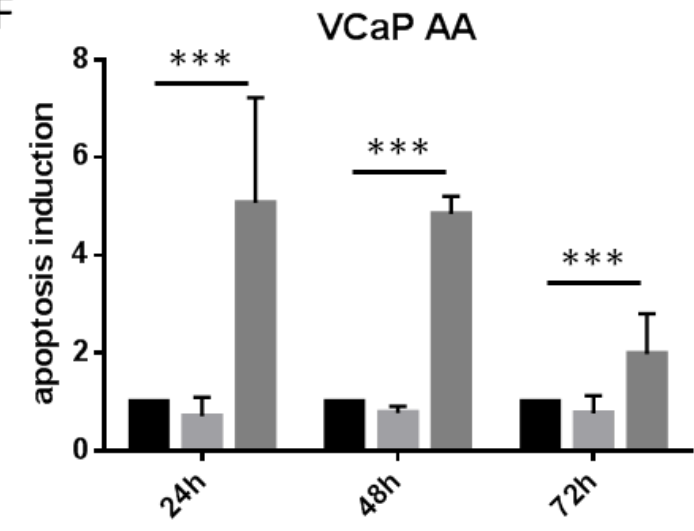

Figure 3.13: Cell survival and induction of apoptosis after $8 \beta$-VE2 treatment in VCaP cell variants. VCaP rev., VCaP and VCaP AA cells were treated with DMSO, $5 \mu \mathrm{M}$ or $25 \mu \mathrm{M} 8 \beta$-VE2 and (A, C and $\mathbf{E}$ ) cell survival and (B, D and F) apoptosis were measured after $24 \mathrm{~h}, 48 \mathrm{~h}$ and $72 \mathrm{~h}$, respectively. All three VCaP cell variants showed a decreased cell survival compared to control cells. In addition, induction of apoptosis could be observed in all VCaP cell variants. Three independent experiments were performed. Data represent the mean \pm s.d. Significance was calculated using the student's t-test. ${ }^{*} p<0.05,{ }^{* *} p<0.01,{ }^{* *} p<0.0001$ compared to DMSO control. 
AR expression and signaling are of utmost importance for $\mathrm{PCa}$ cell survival and progression (Lonergan, Tindall 2011). Therefore, $A R$ mRNA and protein expression and mRNA expression of $A R$-regulated genes after $8 \beta$-VE2 treatment were examined. VCaP rev., VCaP and VCaP AA cells were treated with DMSO, $5 \mu \mathrm{M}$ or $25 \mu \mathrm{M} 8 \beta$-VE2 for $24 \mathrm{~h}$ and RNA samples or proteins were isolated for further studies.

Quantitative RT-PCR and western blot analyses showed that in VCaP and VCaP AA cells the expression of the AR was downregulated significantly upon 8 $\beta$-VE2 treatment (Fig. 3.14 $C, D, E, F)$. In contrast, the marginal expression of AR in VCaP rev. cells was mildly upregulated upon $8 \beta$-VE2 treatment (Fig. 3.14 A, B). As markers for transcriptional activity of the AR the expression of AR-regulated genes IGF1, IGF1R, IGFBP3 and PSA after 8 $\beta$-VE2 treatment was examined. The expression of IGF1, IGF1R and PSA is induced when AR is active, whereas the expression of IGFBP3 is inhibited by AR activation. In 8 $\beta$-VE2-treated VCaP and VCaP AA cells upregulated expression of IGF1 (up to 4.5-fold), IGF1R (up to 2.3fold) and PSA (up to 4.4-fold) and downregulated expression of IGFBP3 (by up to $90 \%$ ) was observed compared to DMSO control (Fig. 3.14 C, E). Furthermore, in VCaP rev cells the expression of IGF1 was downregulated by $50 \%$, IGF1R by $75 \%$ and $P S A$ by $80 \%$ and upregulated for IGFBP3 by 4-fold (Fig 3.14 B). 


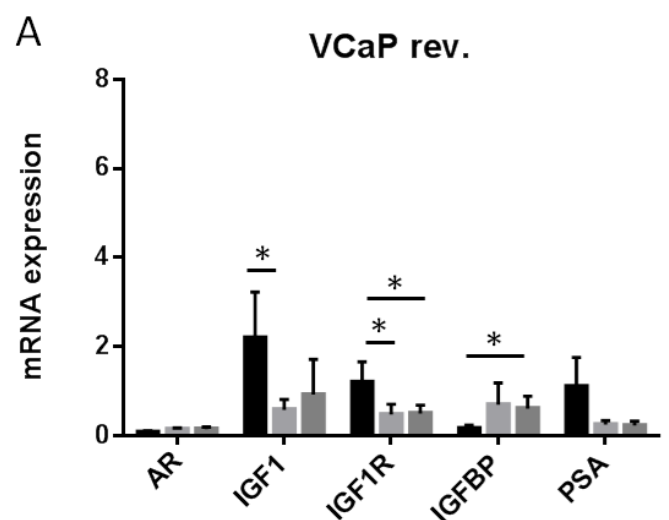

C

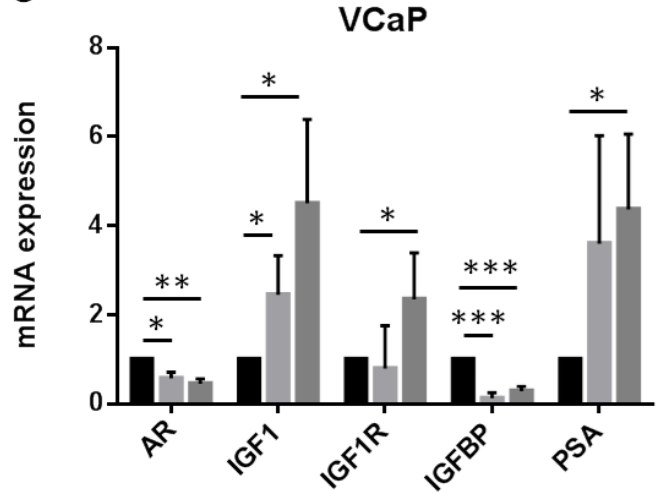

E

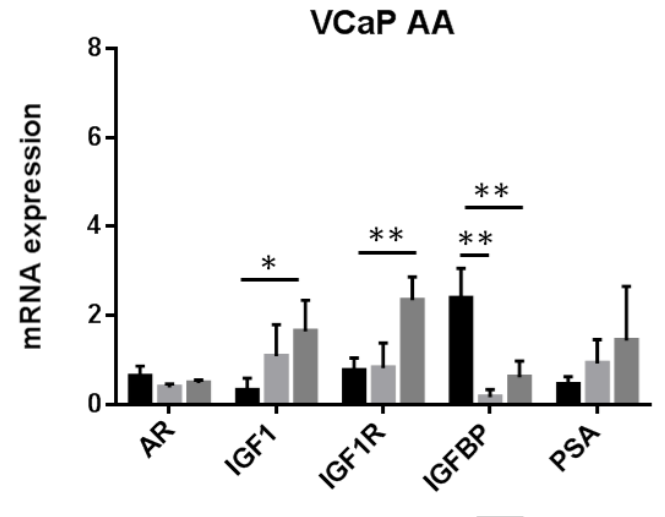

B

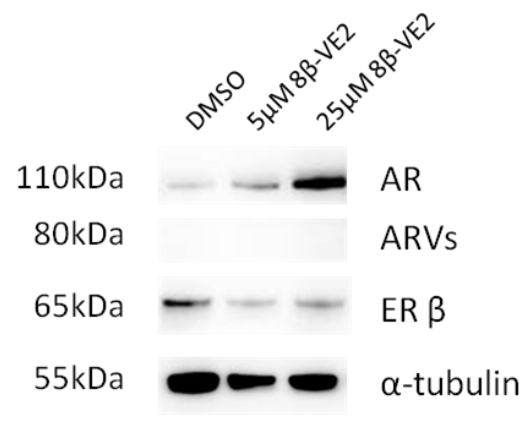

D

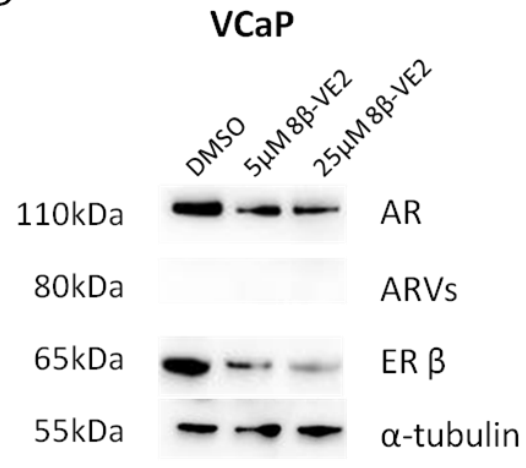

$\mathrm{F}$

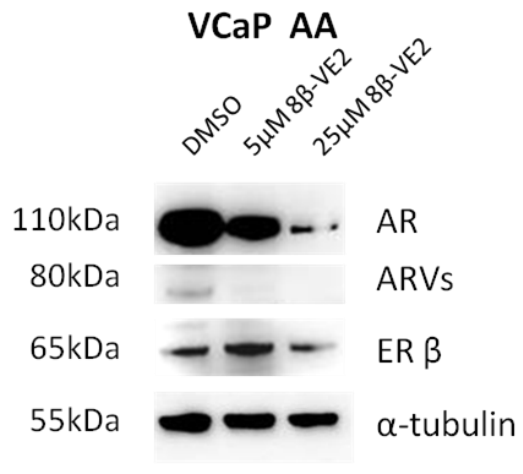

Figure 3.14: $A R$ and AR-regulated gene expression after 8 $\beta$-VE2 treatment in VCaP cell variants. VCaP rev., VCaP and VCaP AA cells were treated with DMSO, $5 \mu \mathrm{M} 8 \beta$-VE2 and $25 \mu \mathrm{M} 8 \beta$ VE2 for $24 \mathrm{~h}$ and RNA and protein were isolated. (A, C and E) AR, IGF1, IGF1R, IGFBP3 and PSA mRNA expression was measured by qRT-PCR. The data represent the mean \pm s.d of two independent experiments, which were performed in duplicate. Significance was calculated using the student's t-test ${ }^{*} p<0.05,{ }^{* *} p<0.01,{ }^{* * *} p<0.0001$ compared with DMSO control. (B, D and F) For protein expression detection AR-specific (first and second panel) and ER $\beta$-specific (third panel) antibodies were used. Representative western blots of two independent experiments performed in duplicates are shown. $\alpha$-tubulin was used as a loading control (lower panel). (A and B) In 8$\beta$-VE2treated VCaP rev. cells an upregulation of $A R$ mRNA and protein expression and a downregulation of AR-regulated gene expression was observed. (C and D) In VCaP cells AR expression was reduced and AR-regulated gene expression was induced after treatment with 8 $\beta$-VE2. (E and F) VCaP AA cells did not show significant expression changes of $A R$ mRNA expression but strong reduction of $A R$ 
protein expression and a remarkable downregulation of AR splice variants (ARVs) expression. The expression of AR-regulated gene expression upon 8 $\beta$-VE2 treatment was induced. In all three cell populations, downregulation of ER $\beta$ protein was observed at high $8 \beta-V E 2$ concentration.

\subsubsection{Whole mRNA-sequencing analysis in VCaP cell variants after $8 \beta-\mathrm{VE} 2$ treatment}

In order to achieve further insights into the molecular mechanism of action leading to the cell survival reduction and induction of apoptosis after $8 \beta$-VE2 treatment, whole mRNAsequencing analysis was performed. All VCaP cell variants were treated with $25 \mu \mathrm{M} 8 \beta$-VE2 or DMSO for $24 \mathrm{~h}$. RNA was prepared and whole mRNA-sequencing analysis was performed in the Transcriptome and Genome Analysis Laboratory (TAL) Microarray and DeepSequencing Facility, University Medical Center Göttingen, using the Illumina system. Data analysis was performed together with the analysts Dr. Thomas Lingner of the TAL facility and Dr. Andreas Leha of the Head Core Facility for Medical Biometry and Statistical Bioinformatics, University Göttingen. Analyses focused on genes with a $\log _{2}$-fold changed expression (see 2.14).

Whole mRNA-sequencing analysis supported the results obtained in previous experiments, e.g. that the VCaP cell variants differ in the expression level of PCa-relevant genes such as the AR. The comparison between the differentially expressed genes of untreated and $8 \beta$ VE2-treated $\mathrm{VCaP}$ cell variants showed that $\mathrm{VCaP}$ and $\mathrm{VCaP}$ rev. cells differ in $10.2 \%$ of all analyzed genes. $\mathrm{VCaP}$ and $\mathrm{VCaP} \mathrm{AA}$ cells differentially expressed $4.8 \%$ of genes and comparison of $\mathrm{VCaP}$ rev. and $\mathrm{VCaP} \mathrm{AA}$ cells revealed differences in the expression of $13.2 \%$ of all expressed genes. Furthermore, 8 $\beta$-VE2-treated VCaP and VCaP AA cells displayed increased similarities to untreated $\mathrm{VCaP}$ rev. cells compared to untreated $\mathrm{VCaP}$ and $\mathrm{VCaP}$ AA cells (Fig 3.15). Especially, VCaP rev. cells after DMSO treatment and $\mathrm{VCaP} A \mathrm{~A}$ cells after $8 \beta-\mathrm{VE} 2$ treatment exhibit greater resemblance than untreated $\mathrm{VCaP}$ rev. and VCaP AA cells ( $8.7 \%$ differentially expressed genes). 


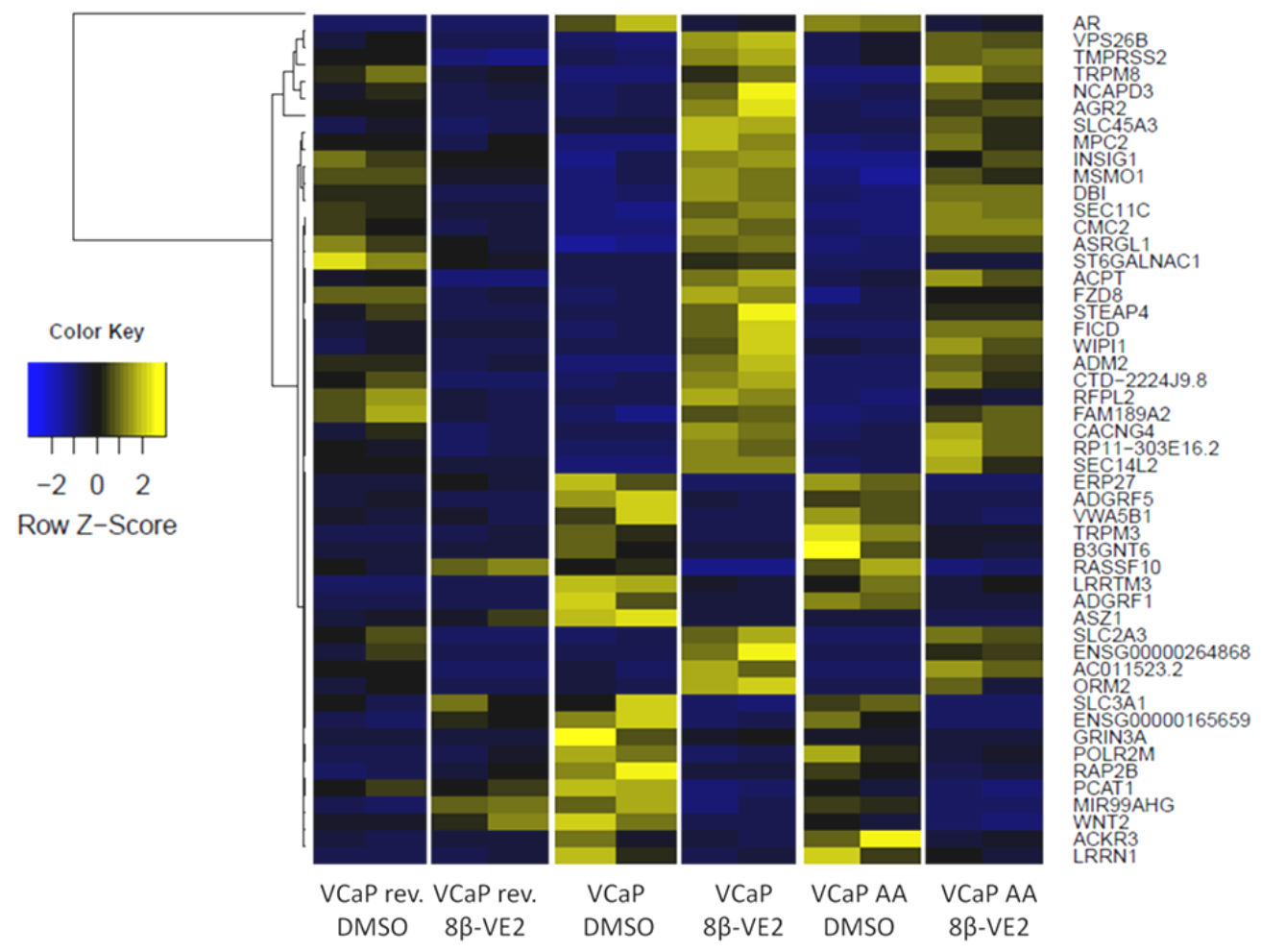

Figure 3.15: Differentially expressed genes in 8 $\beta$-VE2-treated VCaP cell variants. VCaP rev., $\mathrm{VCaP}$ and VCaP AA cells were treated with $25 \mu \mathrm{M} 8 \beta$-VE2 for $24 \mathrm{~h}$ or DMSO as control. RNA was isolated and whole mRNA-sequencing analysis was performed using the Illumina system. The cluster analysis identified 50 genes with the strongest differential expression for DMSO-treated VCaP rev., VCaP and VCaP AA and 8 $\beta$-VE2-treated VCaP rev., VCaP and VCaP AA cells. The data represent three independent experiments. For the analysis two experiments were pooled.

To confirm the whole mRNA-sequencing analysis, qRT-PCR was performed on RNA from the whole mRNA-sequencing experiments for the following genes: WNT2, SHH, MYB, STAT6, PTGER2, FGFR1, SPOCK and EAF2. These genes were chosen because they were previously described to be associated with PCa (Kharaishvili et al. 2011; Srivastava et al. 2012; Statkiewicz et al. 2014; Yang et al. 2013; Yang et al. 2015; Das et al. 2007; Guo et al. 2015; Jain et al. 2008).

Differences in gene expression measured by qRT-PCR were similar to gene expression observed in the whole mRNA-sequencing analysis. The expression was downregulated in 8ß-VE2-treated VCaP and VCaP AA cells up to $80 \%$ for WNT2, up to $92 \%$ for $S H H$, up to $66 \%$ for MYB, up to $75 \%$ for STAT6 and up to $70 \%$ for PTGER2. FGFR1, SPOCK and EAF2 were upregulated in $8 \beta$-VE2-treated $\mathrm{VCaP}$ and $\mathrm{VCaP} A \mathrm{~A}$ cells up to 5 -fold for FGFR1, up to 5.9-fold for SPOCK and up to 3.4-fold for EAF2 (Fig. 3.16 A, B). In 8ß-VE2-treated $\mathrm{VCaP}$ rev. cells most genes were reversely regulated analogous to the whole mRNAsequencing data. The expression of WNT2 (2.5-fold), SHH (4-fold), MYB (1.7-fold) and 
PTGER2 (2-fold) was upregulated. The expression of SPOCK (50\%) and EAF2 (40\%) was downregulated. STAT6 and FGFR1 expression did not change after treatment (Fig. $3.16 \mathrm{C}$ ).

A

VCaP rev.
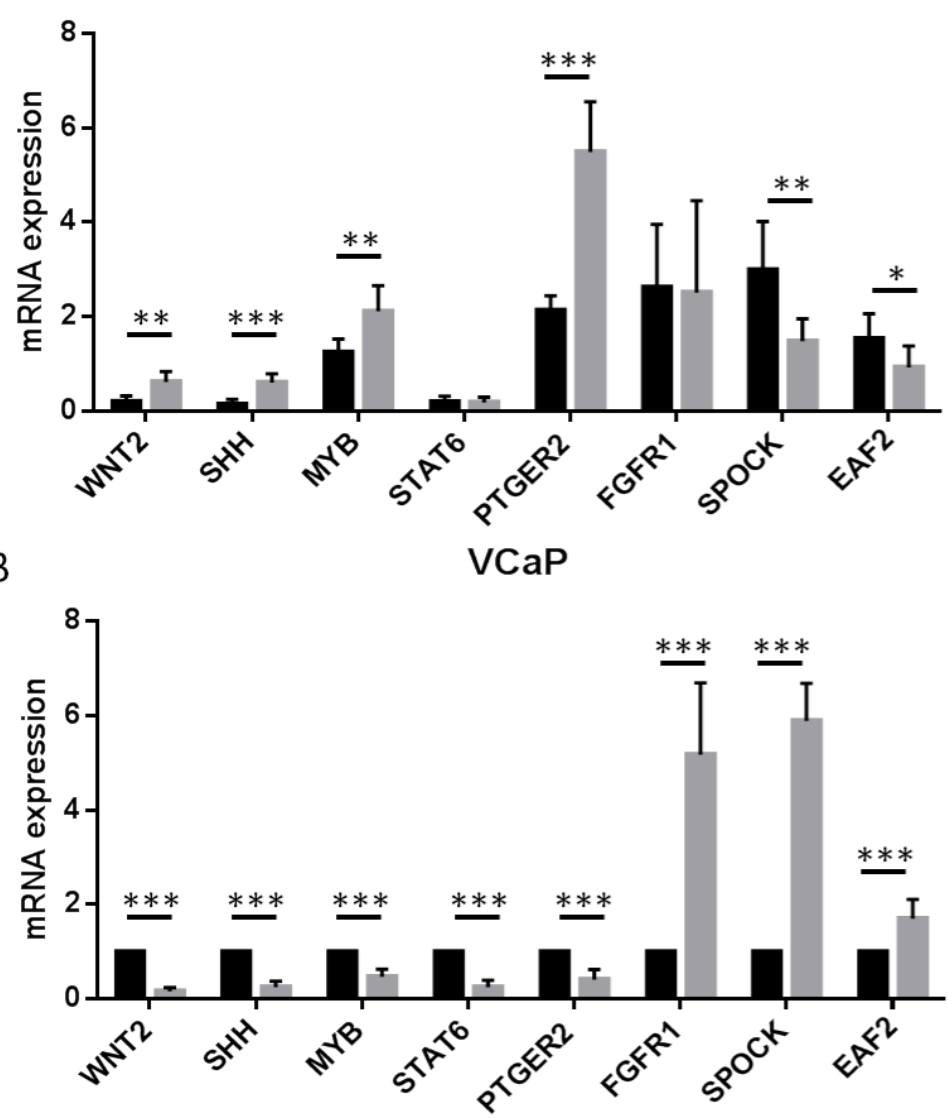

C

VCaP AA

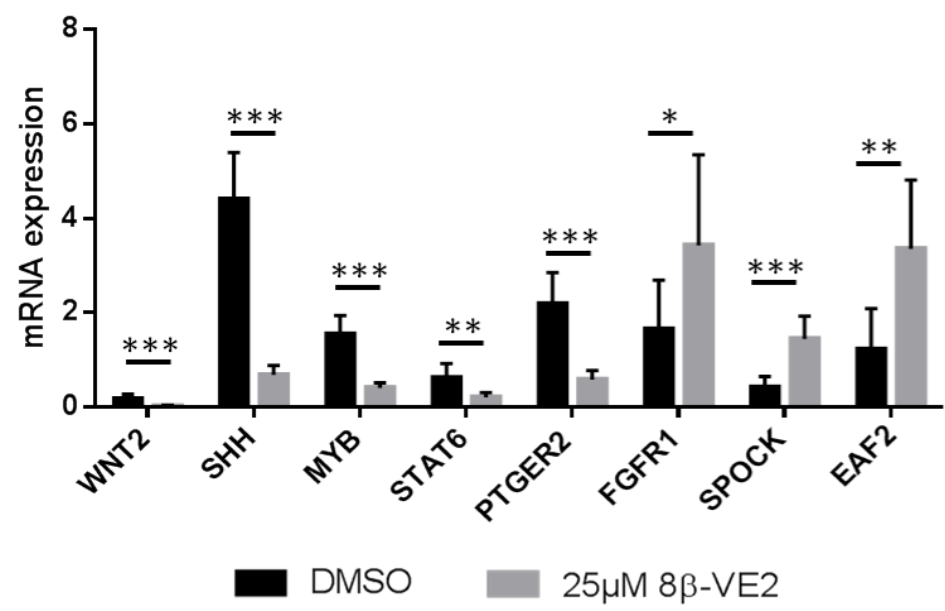

Figure 3.16: Confirmation of differentially expressed genes after whole mRNA-sequencing by qRT-PCR analysis. (A) VCaP rev., (B) VCaP and (C) VCaP AA cells were treated with DMSO or $25 \mu \mathrm{M} 8 \beta$-VE2 for $24 \mathrm{~h}$. RNA from whole mRNA-sequencing analysis was used to analyze gene expression by qRT-PCR analysis. All expression profiles concurred to the data generated in the whole mRNA-sequencing analysis. Data represent the mean $\pm \mathrm{s} . \mathrm{d}$ of three independent experiments, which were performed in duplicate. Significance was calculated using the student's t-test. ${ }^{*} p<0.05$, ** $p<0.01,{ }^{* * *} p<0.0001$ compared with DMSO control. 
Table 3.1: Overview of differential gene expression of candidate genes identified by whole mRNA-sequencing in 8 $\beta$-VE2- and DMSO-treated VCaP rev., VCaP and VCaP AA cells. $(+)=$ increased expression; $(-)=$ decreased expression; n.s. $=$ not significant

\begin{tabular}{l|c|c|c}
\hline Gene name & VCaP rev. & VCaP & VCaP AA \\
\hline WNT2 & + & - & - \\
SHH & + & - & - \\
MYB & + & - & - \\
STAT6 & n.s. & - & - \\
PTGER2 & + & - & - \\
FGFR1 & n.s. & + & + \\
SPOCK & - & + & + \\
EAF2 & - & + & +
\end{tabular}

Furthermore, whole mRNA-sequencing analysis also confirmed the reduction of $A R$ and $A R V$ expression which was previously observed by western blot analysis (see Fig. 3.14). $A R$ specific reads decreased in VCaP cells by $79 \%$ and VCaP AA cells by $75 \%$ after $8 \beta-\mathrm{VE} 2$ treatment and unchanged in VCaP rev. cells (Fig. 3.17 A). ARV mRNA expression was reduced in all VCaP cell variants; in VCaP rev. no $A R V$ specific reads could be detected after 8ß-VE2 treatment. In VCaP and VCaP AA cells ARV expression was reduced by app. $96 \%$ compared to control treatment (Fig. 3.17 B). 


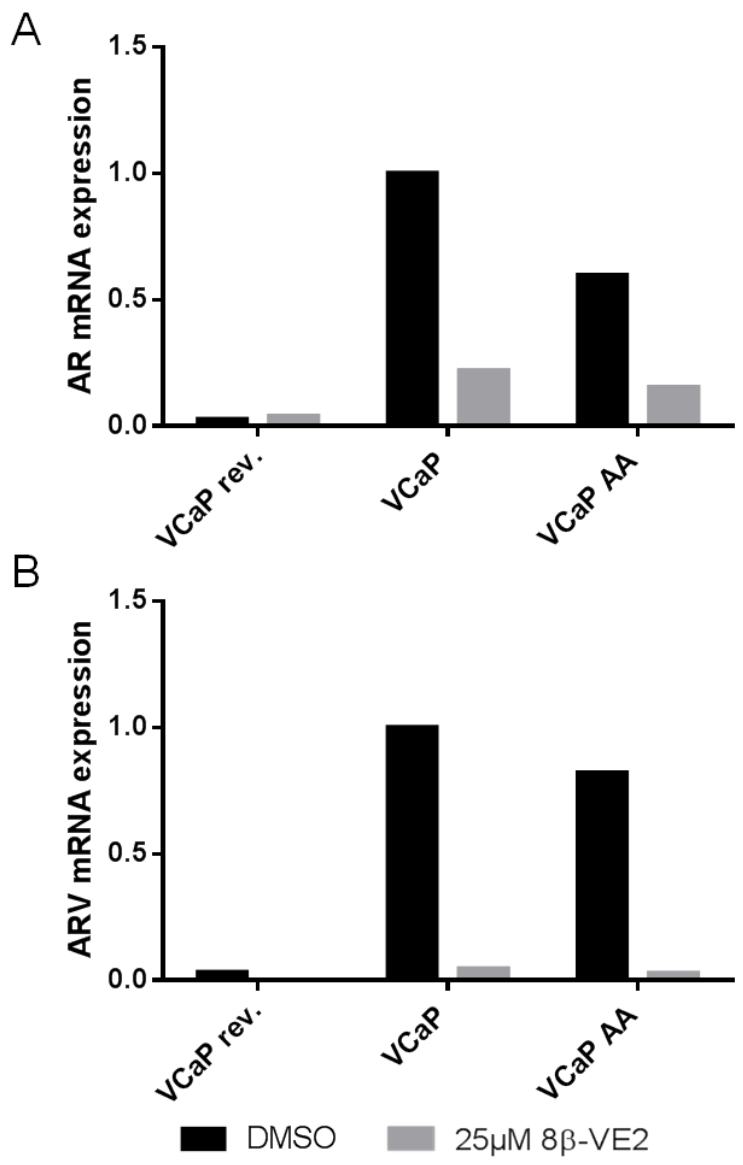

Figure 3.17: Expression of $A \boldsymbol{R}$ and $\boldsymbol{A R V s}$ in $8 \beta$-VE2-treated VCaP cell variants. Whole mRNAsequencing analysis demonstrated that (A) $A R$ and (B) $A R V$ expression is downregulated in $\mathrm{VCaP}$ and $\mathrm{VCaP} A \mathrm{~A}$ cells after $8 \beta$-VE2 treatment. Data represent three independent experiments. For the analysis two experiments were pooled. Depicted are counts per million reads (CPM) relative to DMSOtreated VCaP cells.

In contrast to the western blot analyses, whole mRNA-sequencing data indicated that ER $\beta$ is only weakly expressed in VCaP cell variants. Whole mRNA-sequencing data were screened for ER $\beta$ target gene expression as an indicator for ER $\beta$ activation due to $8 \beta$-VE2 treatment, however to date, only a few ER $\beta$ target genes are described in the literature and the majority of publications described ER $\beta$ target genes in breast cancer (Williams et al. 2008). Interestingly, Vivar et al. also depicted genes expressed after ER $\beta$ overexpression in PC3 PCa cells. Genes delineated by Vivar et al. (2010) and Williams et al. (2008) were compared to differentially expressed genes in $8 \beta$-VE2-treated VCaP cell variants. Surprisingly, neither the gene expression pattern of ER $\beta$ overexpressing PC3 cells nor of ER $\beta$ agonist-treated breast cancer cells showed significant concurrence to $8 \beta$-VE2-treated VCaP cell variants (commonly expressed genes $>1 \%$ of all differentially expressed genes). Furthermore, no matches were found in the pathway analysis between described ER $\beta$-activated pathways and pathways activated in $8 \beta-\mathrm{VE} 2$-treated VCaP cell variants. 


\subsubsection{Analysis of the role of ER $\beta$ in $8 \beta$-VE2-mediated treatment effects}

Whole mRNA-sequencing analysis and protein detection could not give information about ER $\beta$ activation upon 8$\beta$-VE2 treatment. Hence, the two ER $\beta$ agonists ERB 041 and FERb 033 were used to elucidate the role of ER $\beta$ activation in PCa cells and investigate if $8 \beta$-VE2 might exhibit a special function. ERB 041 belongs to the benzoxazolol class and displays 200-fold selectivity for ER $\beta$ over ER $\alpha$ (Minutolo et al. 2009). FERb 033 belongs to the carboxaldehyde oxime and displays 62 -fold specificity for ER $\beta$ over ER $\alpha$ (Malamas et al. 2004).

\subsection{Analyses of cellular effects of ERB 041 treatment on VCaP cell variants}

In order to analyze the effect of ERB 041 on VCaP variants, cell survival, induction of apoptosis and expression of the AR and AR-regulated genes was examined. VCaP rev., $\mathrm{VCaP}$ and $\mathrm{VCaP}$ AA cells were treated with $5 \mu \mathrm{M}$ and $25 \mu \mathrm{M}$ ERB 041 or DMSO as control for $24 \mathrm{~h}, 48 \mathrm{~h}$ and $72 \mathrm{~h}$ for measurement of living cells, dead cells as well as caspase activity. VCaP rev., VCaP and VCaP AA cells were treated with $5 \mu \mathrm{M}$ and $25 \mu \mathrm{M}$ ERB 041 or DMSO as control for $24 \mathrm{~h}$ for RNA and protein isolation.

ERB 041 treatment led to significant reduction of cell survival in VCaP cells ( $37 \%$ after $72 \mathrm{~h}$ ) (Fig $3.18 \mathrm{C}$ ), but did not reduce the cell survival in VCaP rev. or VCaP AA cells (Fig. $3.18 \mathrm{~A}$, E). No apoptosis induction was observed in any of the VCaP cell variants analyzed. In contrast, apoptosis was decreased in ERB 041-treated cells (Fig. 3.18 B, D, F). 
A

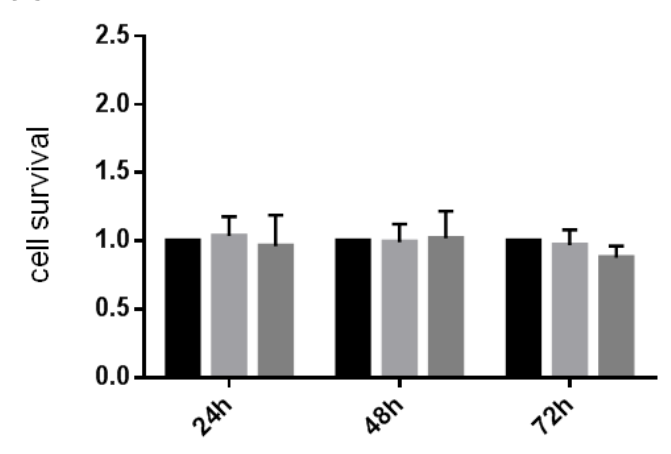

C

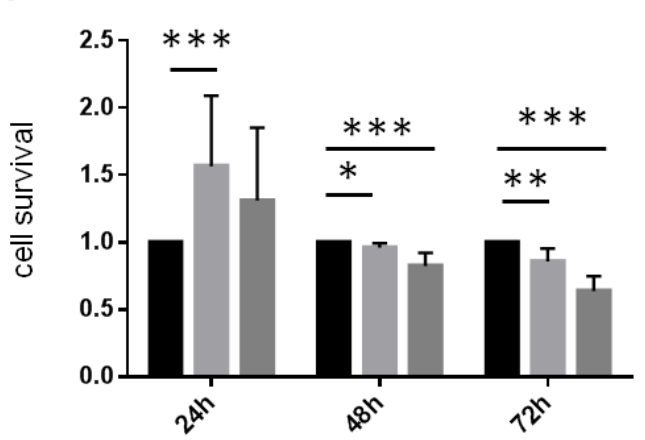

$\mathrm{E}$

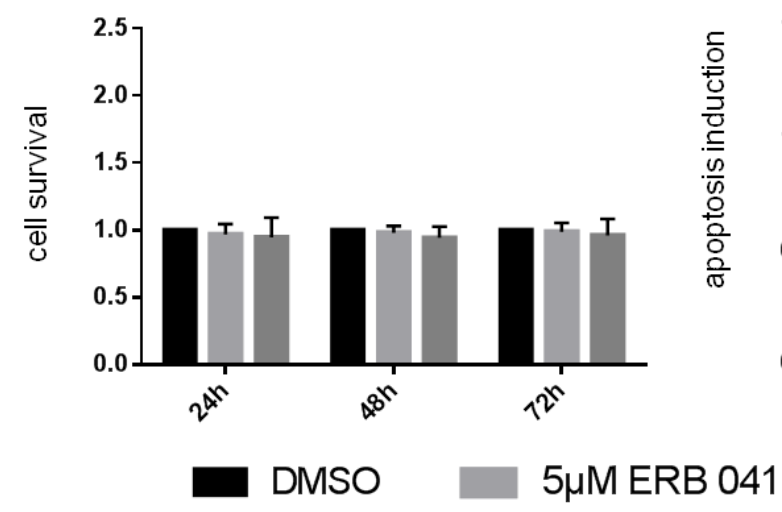

B

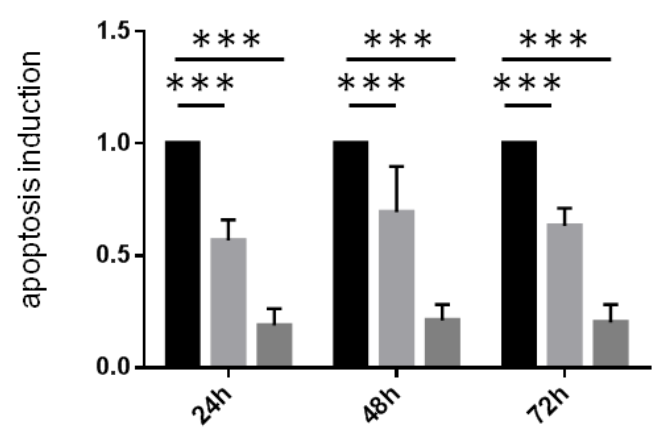

D

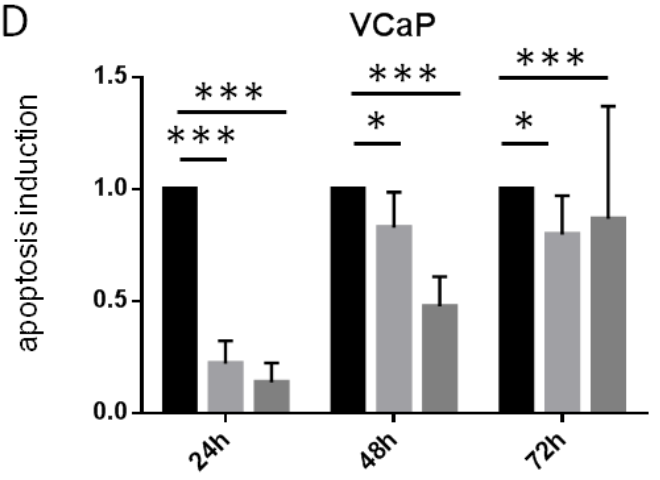

$\mathrm{F}$

VCaP AA

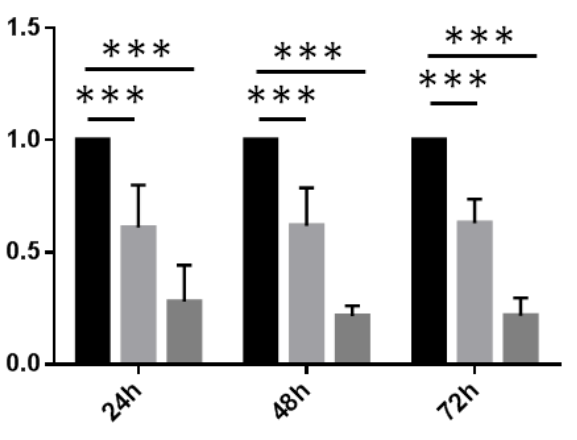

$25 \mu M$ ERB 041

Figure 3.18: Cell survival and apoptosis induction in ERB 041-treated VCaP cell variants. VCaP rev., VCaP and VCaP AA cells were treated with $5 \mu \mathrm{M}$ or $25 \mu \mathrm{M}$ ERB 041 or DMSO as control. (A, C, E) Cell survival and (B, D, F) induction of apoptosis were measured after $24 \mathrm{~h}, 48 \mathrm{~h}$ and $72 \mathrm{~h}$. (C) Significant cell survival reduction could only be observed in VCaP cells, strongest effect was seen after $72 \mathrm{~h}$ of treatment with $25 \mu \mathrm{M}$ ERB 041 by $37 \%$. Induction of apoptosis was decreased in all three cell lines upon ERB 041 treatment. Data represent mean \pm s.d. of three independent experiments, which were performed in duplicate. Significance was calculated using the student's t-test ${ }^{*} p<0.05$, ${ }^{* *} p<0.01,{ }^{* * *} p<0.0001$ compared with DMSO control.

MRNA and protein analyses revealed that AR expression was not increased in VCaP rev. and not reduced in VCaP and VCaP AA cells upon ERB 041 treatment as it was shown after 8 $\beta$-VE2 treatment (Fig. 3.19). Only $5 \mu \mathrm{M}$ ERB 041-treated VCaP AA cells showed mild reduction of $A R$ expression of $9 \%$ (Fig. $3.19 \mathrm{E}, \mathrm{F}$ ). The expression of AR-regulated genes was mainly unchanged after ERB 041 treatment. 
A

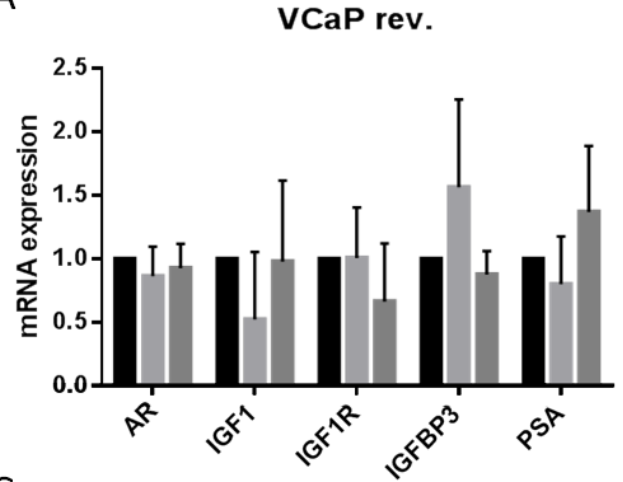

C

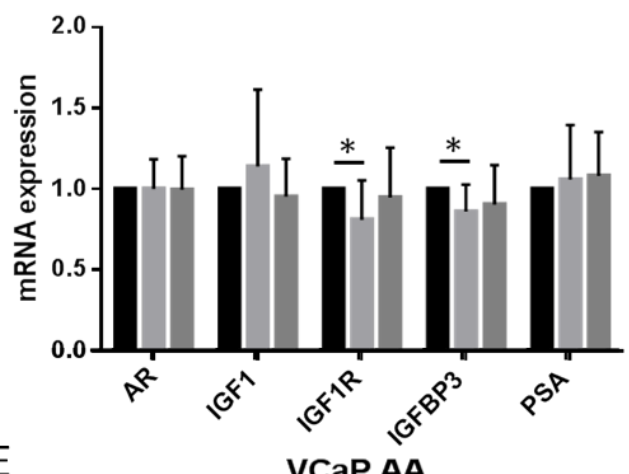

$\mathrm{E}$

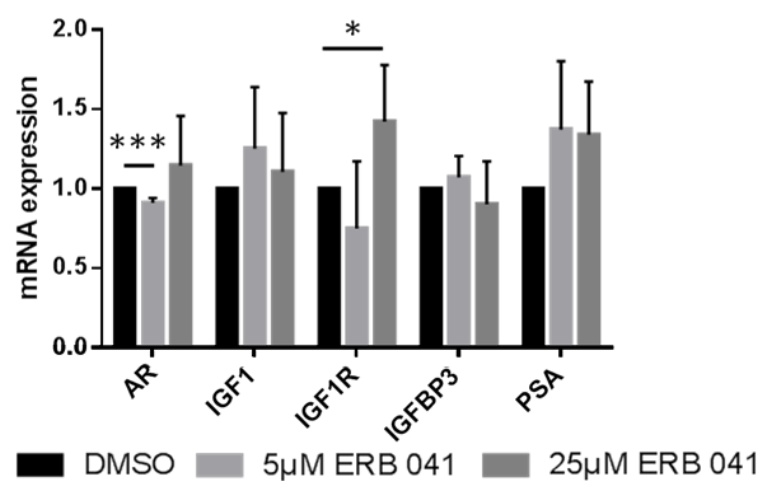

B

VCaP rev.

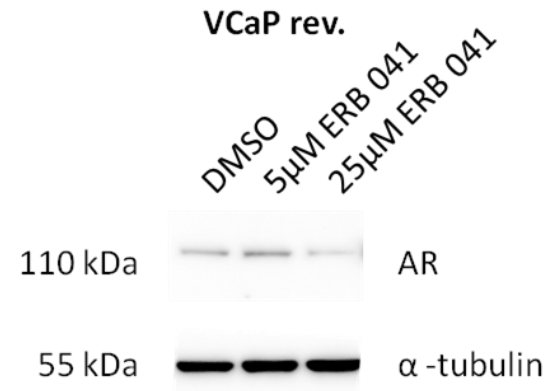

D

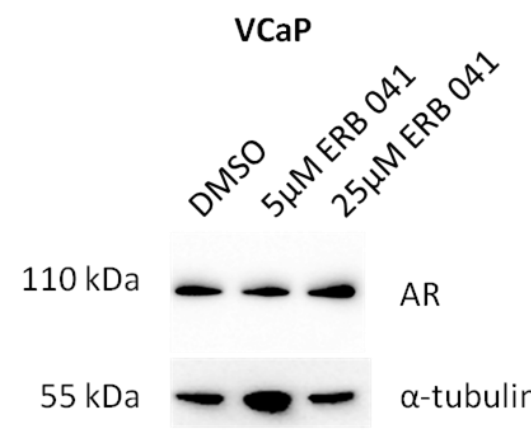

VCaP AA

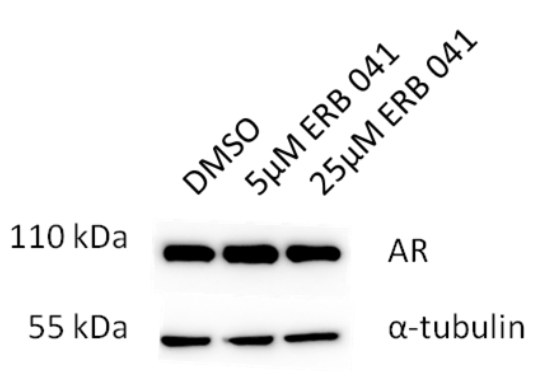

Figure 3.19: $A R$ and $A R$ target gene expression after ERB 041 treatment in VCaP cell variants.

VCaP rev., VCaP and VCaP AA cells were treated with $5 \mu \mathrm{M}$ or $25 \mu \mathrm{M}$ ERB 041 or DMSO as control. After $24 \mathrm{~h}$ RNA and protein were isolated and both $\mathrm{qRT}-\mathrm{PCR}$ and western blotting analyses were performed. (A, C and E) Neither $A R$ nor the AR-regulated genes displayed changed mRNA expression due to ERB 041 treatment in the three VCaP cell variants. Only VCaP AA cells showed a $9 \%$ reduced $A R$ expression. Data represent the mean \pm s.d. of two independent experiments, which were performed in duplicate. Significance was calculated using the student's t-test ${ }^{*} p<0.05,{ }^{* *} p<0.01$, ${ }^{* * *}$ $\mathrm{p}<0.0001$ compared to DMSO control. (B, $\mathbf{D}$ and $\mathbf{F}$ ) No changes in AR protein expression (upper panel) could be detected in the three $\mathrm{VCaP}$ cell variants. A representative western blot of two independent experiments performed in duplicates is shown. $\alpha$-tubulin was used as loading control (lower panel). 
Taken together, ERB 041 treatment reduced cell survival in VCaP cells, but also decreased apoptosis. Furthermore, treatment of $\mathrm{VCaP}$ cell variants with ERB 041 did not lead to a reduction of $A R$ expression and induction of $A R$-regulated gene expression.

\subsection{Analyses of cellular effects of FERb 033 treatment on VCaP cell variants}

In order to analyze the effect of FERb 033 on VCaP variants, again cell survival, induction of apoptosis and expression of the AR and AR-regulated genes was examined. VCaP rev., VCaP and VCaP AA cells were treated with $25 \mu \mathrm{M}$ and $50 \mu \mathrm{M}$ FERb 033 or DMSO as control for $24 \mathrm{~h}, 48 \mathrm{~h}$ and $72 \mathrm{~h}$ for measurement of living cells, dead cells as well as caspase activity. VCaP rev., VCaP and VCaP AA cells were treated with $25 \mu \mathrm{M}$ and $50 \mu \mathrm{M}$ FERb 033 or DMSO as control for $24 \mathrm{~h}$ for RNA and protein isolation.

FERb 033 treatment led to a significant reduction of cell survival in all three VCaP cell variants tested. Strongest effects were observed in VCaP rev. cells after treatment with $50 \mu \mathrm{M}$ FERb 033 for $72 \mathrm{~h}$ (24\% reduction) (Fig $3.20 \mathrm{~A}$ ), in VCaP and VCaP AA cells after $48 \mathrm{~h}$ treatment with $50 \mu \mathrm{M}$ FERb 033 (54\% reduction in VCaP cells and $47 \%$ reduction in VCaP AA cells) (Fig. 3.20 C, E). Apoptosis induction was observed in all three VCaP cell variants. VCaP rev. cells showed 1.8-fold increased apoptosis after 72 h (Fig. 3.20 B). VCaP cells displayed induction of apoptosis after treatment with $25 \mu \mathrm{M}$ or $50 \mu \mathrm{M}$ FERb 033 for $24 \mathrm{~h}$ or $48 \mathrm{~h}$ (up to 3-fold) (Fig. 3.20 D). VCaP AA cells exhibited early induction of apoptosis (1.7fold) after treatment with $25 \mu \mathrm{M}$ or $50 \mu \mathrm{M}$ FERb 033 for $24 \mathrm{~h}$ (Fig $3.20 \mathrm{~F}$ ). 
A

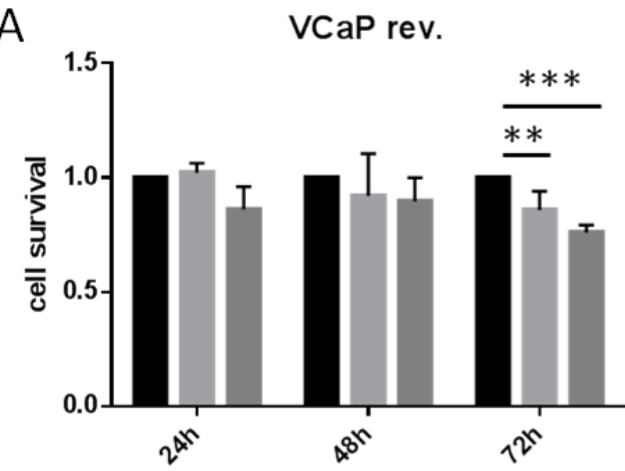

C

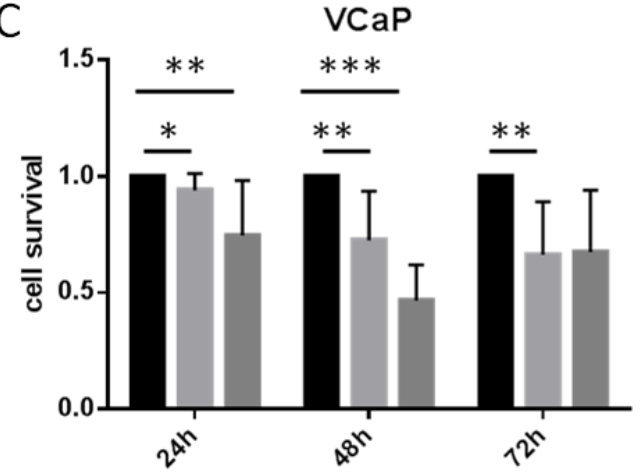

E

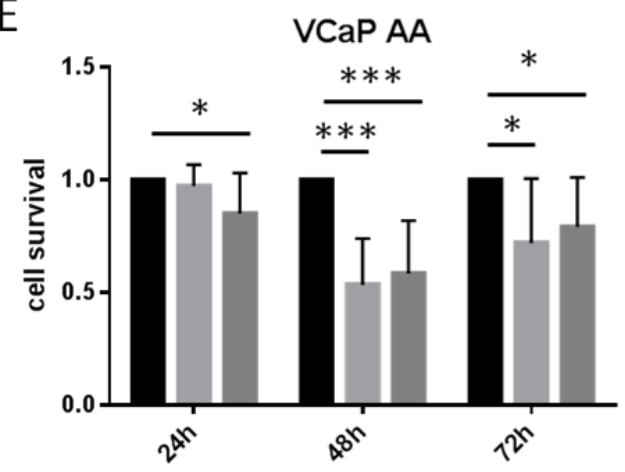

B

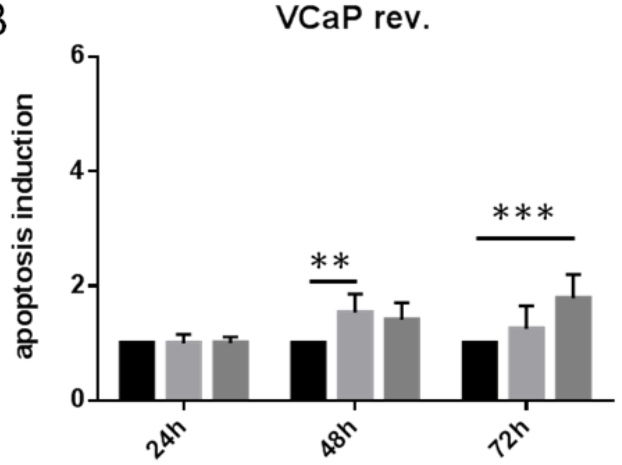

D

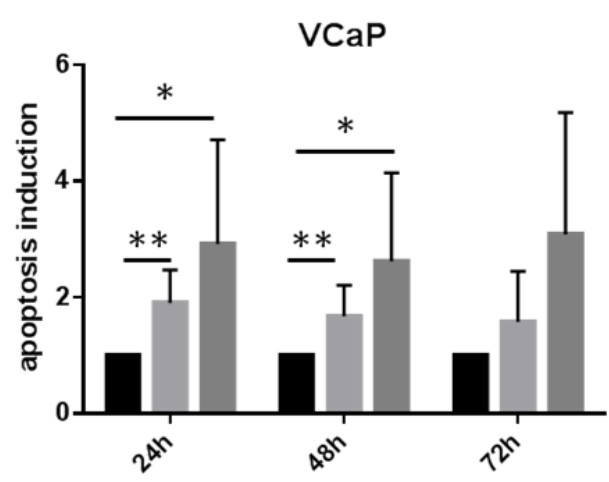

F

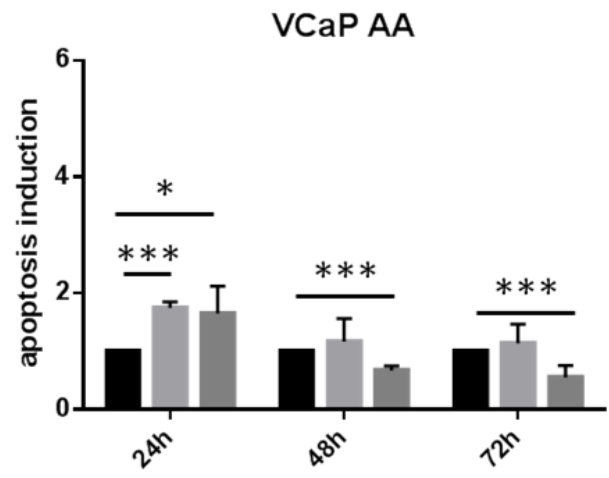

DMSO

25HM FERb 033

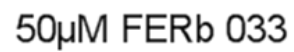

Figure 3.20: Cell survival and apoptosis induction in FERb 033-treated VCaP cell variants. VCaP rev., VCaP and VCaP AA cells were treated with $25 \mu \mathrm{M}$ or $50 \mu \mathrm{M}$ FERb 033 or DMSO as control. (A, C, E) Cell survival and (B, D, F) induction of apoptosis were measured after $24 \mathrm{~h}, 48 \mathrm{~h}$ and $72 \mathrm{~h}$. VCaP rev. cells showed late response to FERb 033 with (A) $24 \%$ cell survival reduction and (B) 1.8-fold apoptosis induction after $72 \mathrm{~h}$ treatment. VCaP cells showed strongest response to FERb 033 after $48 \mathrm{~h}$ treatment with (C) $54 \%$ cell survival reduction and (D) 3-fold apoptosis induction. VCaP AA cells showed early response to FERb 033 and (E) strongest cell survival reduction (47\%) and (F) apoptosis induction (1.7-fold) after $24 \mathrm{~h}$. Data represent the mean \pm s.d. of three independent experiments, which were performed in duplicate. Significance was calculated using the student's t-test. ${ }^{*} p<0.05,{ }^{* *} p<0.01,{ }^{* * *} p<0.0001$ compared with DMSO control. 
In FERb 033-treated cells, qRT-PCR analysis showed that $A R$ expression was decreased in VCaP rev. cells (by $50 \%$ in $25 \mu \mathrm{M}$ FERb 033-treated cells) (Fig $3.21 \mathrm{~A}$ ), but $A R$ expression was not reduced in VCaP and VCaP AA cells upon FERb 033 treatment (Fig 3.21 C, E). The expression of AR-regulated genes was downregulated in VCaP rev. cells (IGF1 by $45 \%$, IGF1R by $31 \%$ ) (Fig $3.21 \mathrm{~A}$ ) and not affected in VCaP cells after FERb 033 treatment (Fig $3.21 \mathrm{C})$. In VCaP AA cells the expression of AR-regulated genes was decreased after $25 \mu \mathrm{M}$ FERb 033, but upregulated upon treatment with $50 \mu \mathrm{M}$ FERb 033 (Fig $3.21 \mathrm{E}$ ). AR protein expression was not changed after FERb 033 treatment. Treatment with $50 \mu \mathrm{M}$ FERb 033induced protein degradation in VCaP AA cells (Fig 3.21 B, D, F). 
A

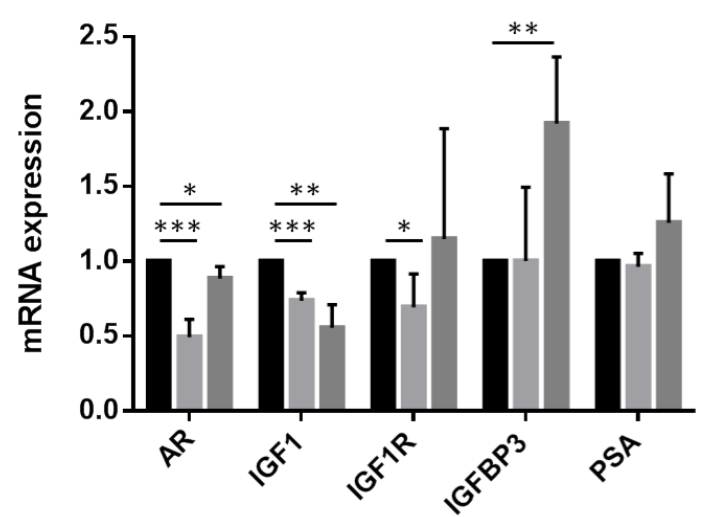

C

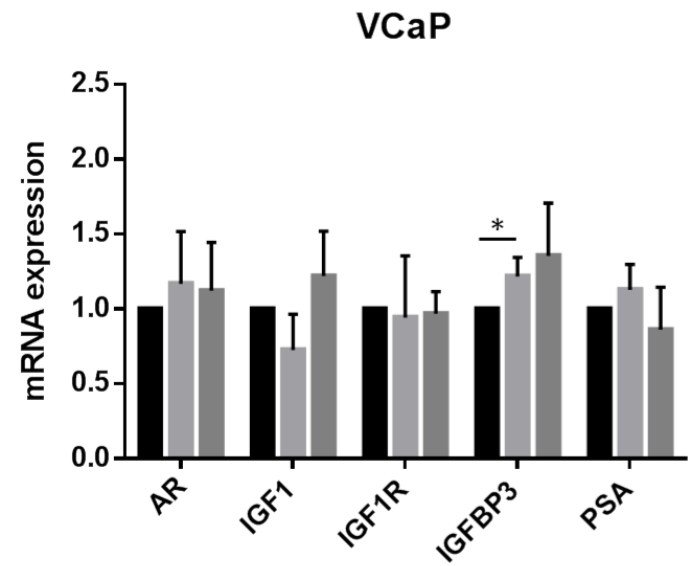

E

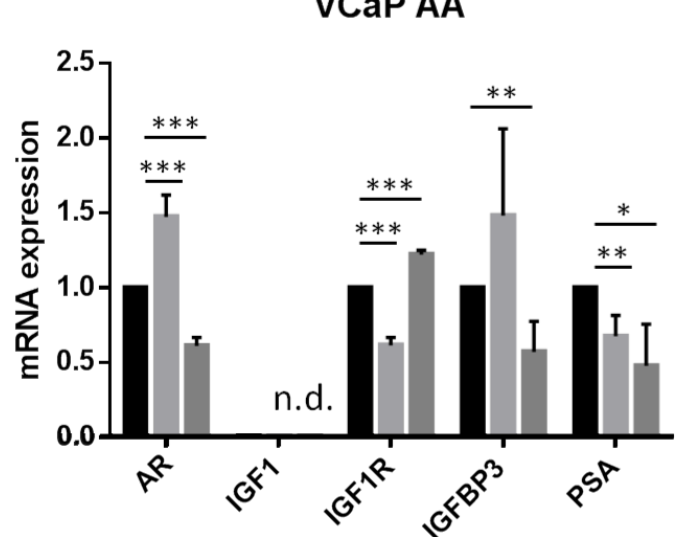

B

VCaP rev.

$110 \mathrm{kDa}$

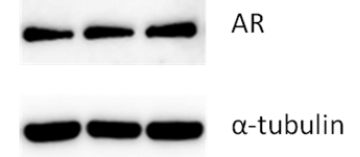

D

VCaP

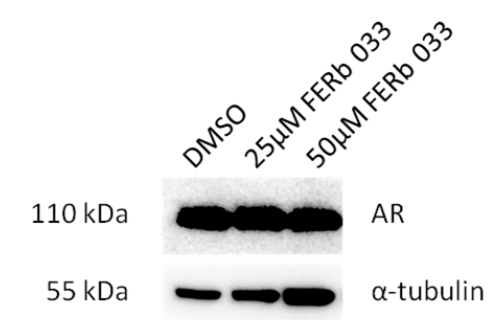

F

VCaPAA

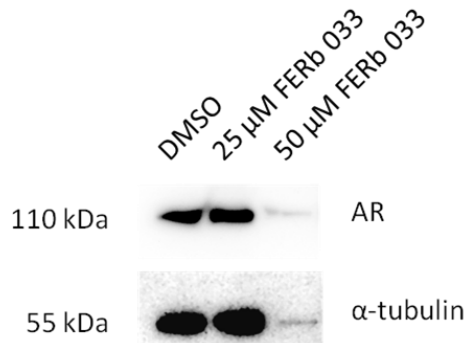

Figure 3.21: AR and AR target gene expression after FERb 033 treatment in VCaP cell variants. VCaP rev., VCaP and VCaP AA cells were treated with $25 \mu \mathrm{M}$ or $50 \mu \mathrm{M}$ FERb 033 or DMSO as control for $24 \mathrm{~h}$. No consistent effect could be observed in mRNA expression of the $A R$ and ARregulated genes. (A and $\mathbf{B}$ ) VCaP rev. cells showed downregulated $A R$ expression and downregulation of $A R$ target gene expression in RNA analysis but no effect on AR protein expression (upper panel). (C and D) VCaP cells showed no effects on gene expression after FERb 033 treatment. (E and F) VCaP AA cells showed increased $A R$ mRNA expression and decreased expression of ARregulated genes upon treatment with $25 \mu \mathrm{M}$ FERb 033, but no changes of AR protein expression. Decreased $A R$ expression and increased $A R$ target gene expression was observed upon treatment with $50 \mu \mathrm{M}$ FERb 033. However, treatment with $50 \mu \mathrm{M}$ FERb 033 induced protein degradation (upper 
panel). The data represent the mean \pm s.d. of two independent experiments, which were performed in duplicate. Significance was calculated using the student's t-test. ${ }^{*} p<0.05,{ }^{* *} p<0.01,{ }^{* \star *} p<0.0001$ compared to DMSO control. A representative western blot of two independent experiments which were performed in duplicates is shown. $\alpha$-tubulin was used as a loading control (lower panel).

In summary, the effects on $\mathrm{VCaP}$ cell variants induced by FERb 033 treatment are more similar to the effects observed after $8 \beta$-VE2 treatment than after ERB 041 treatment, i.e.

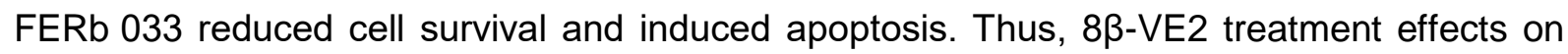
VCaP cell variants were stronger and more pronounced. Furthermore, FERb 033 treatment of $\mathrm{VCaP}$ cell variants could not replicate the specific reduction of $A R$ expression and induction of AR-regulated gene expression observed after cellular 8 $\beta$-VE2 treatment. Therefore, these findings indicate that either the $8 \beta$-VE2-induced effects in VCaP cell variants are not ER $\beta$-dependent or mediated by an alternative ER $\beta$ pathway which is not activated by the two other ER $\beta$ agonists.

\subsubsection{Analyses of alternative ER $\boldsymbol{\beta}$-mediated signaling pathways}

The whole mRNA-sequencing analysis did not reveal the expression of known ER $\beta$ regulated genes after $8 \beta$-VE2 treatment in VCaP cell variants. Therefore, $8 \beta$-VE2 might not increase transcriptional activity of $E R \beta$, but activate alternative ER $\beta$-mediated signaling pathways. Alternative ER $\beta$ signaling can be activated by membrane-bound ER $\beta$ employing second messenger signaling. Subsequently, second messengers can activate the expression of genes without estrogen response elements or lead to non-transcriptional effects of ER $\beta$ activation (see Fig. 1.3) (Cui et al. 2013). To investigate if alternative ER $\beta$ signaling is involved in the $8 \beta$-VE2-induced effects, VCaP cells were treated with $8 \beta$-VE2 and the ER $\beta$ inhibitor fulvestrant. Fulvestrant is an ER $\beta$ antagonist without partial agonistic activity. It inhibits ligand binding, dimerisation and facilitates ER $\beta$ degradation (Peekhaus et al. 2004). Therefore, $8 \beta$-VE2 together with fulvestrant treatment should inhibit transcriptional active ER $\beta$ as well as alternative ER $\beta$-mediated signaling in VCaP cells.

The effect of combined treatment with $8 \beta$-VE2 and fulvestrant on cell survival, apoptosis induction and gene expression was investigated. VCaP cells were treated with either $1 \mu \mathrm{M}$ fulvestrant, the combination of fulvestrant and $8 \beta$-VE2 or DMSO as control. Cell survival and apoptosis induction were measured after $24 \mathrm{~h}, 48 \mathrm{~h}$ and $72 \mathrm{~h}$. RNA for expression analysis was isolated after $24 \mathrm{~h}$.

Treatment of VCaP cells with $1 \mu \mathrm{M}$ fulvestrant reduced cell survival after $72 \mathrm{~h}$ by $12 \%$ and it induced apoptosis after $48 \mathrm{~h}$ by 1.7 -fold compared to DMSO-treated control cells. The combined treatment with $25 \mu \mathrm{M} 8 \beta$-VE2 and $1 \mu \mathrm{M}$ fulvestrant led to a reduced cell survival (47\%) and induced apoptosis (3.9-fold) comparable to the effects observed with $8 \beta$-VE2 treatment only (see 3.1.2.2). The blockade of ER $\beta$ by the ER $\beta$ antagonist fulvestrant could 
not inhibit the treatment effects of $8 \beta$-VE2 although cell survival reduction was slightly reduced and apoptosis was only induced about 4-fold instead of 5.3-fold without fulvestrant (Fig. 3.22). Therefore, these results indicate that $8 \beta$-VE2-induced effects are not exclusively mediated by ER $\beta$ in VCaP cells.

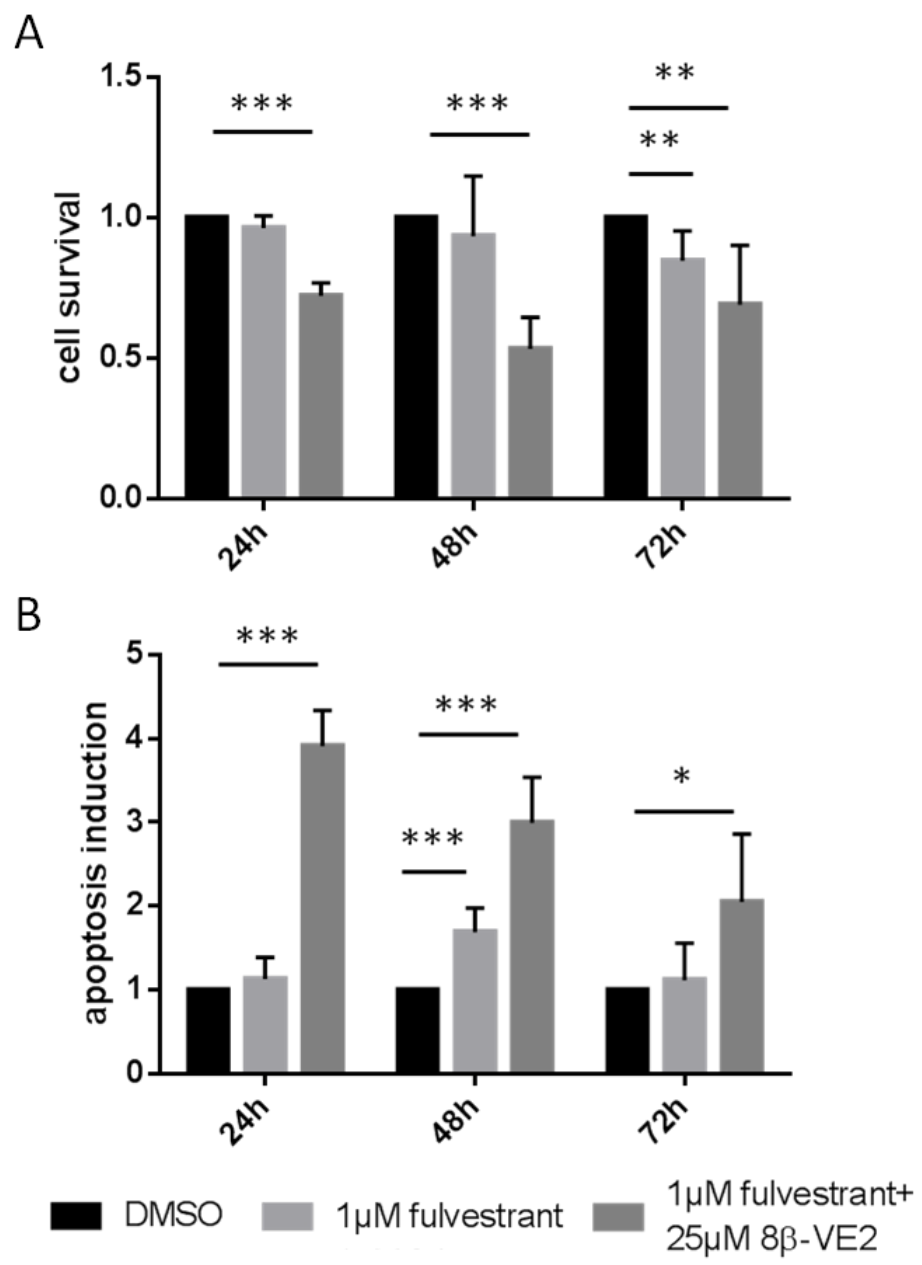

Figure 3.22: Cell survival and apoptosis induction in VCaP cells upon $8 \beta$-VE2 and fulvestrant treatment. VCaP cells were treated with $25 \mu \mathrm{M}$ of the ER $\beta$ agonist $8 \beta$-VE2 together with $1 \mu \mathrm{M}$ of the ER $\beta$ antagonist fulvestrant to investigate if $8 \beta$-VE2-induced cell survival reduction and apoptosis induction are ER $\beta$-mediated. Simultaneous treatment with $8 \beta$-VE2 and fulvestrant-induced (A) cell survival reduction of $31 \%$ and $(B)$ apoptosis induction up to 4 -fold compared to DMSO control. $8 \beta$ VE2-mediated treatment effects could not be blocked by ER $\beta$ inhibition. The data represent the mean \pm s.d. of three independent experiments, which were performed in duplicate. Significance was calculated using the student's t-test ${ }^{*} p<0.05,{ }^{* *} p<0.01,{ }^{* * *} p<0.0001$ compared to DMSO control.

To analyze the effect of combined $8 \beta$-VE2 and fulvestrant treatment on $A R$ and AR-regulated gene expression qRT-PCR analyses were performed.

The simultaneous treatment with $8 \beta$-VE2 and fulvestrant did not diminish the treatment effects observed after single $8 \beta-\mathrm{VE} 2$ treatment. Combined treatment with $8 \beta-\mathrm{VE} 2$ and fulvestrant reduced $A R$ expression by $53 \%$ and induced expression of IGF1 by 3.7-fold, IGF1R expression by 2.4 -fold and PSA expression by 2.5 -fold. IGFBP3 expression 
decreased without fulvestrant by $54 \%$ and with fulvestrant by $18 \%$ (Fig. 3.23). Taken together, these data implicate that the induction of AR-regulated gene expression is not solely ER $\beta$-mediated.

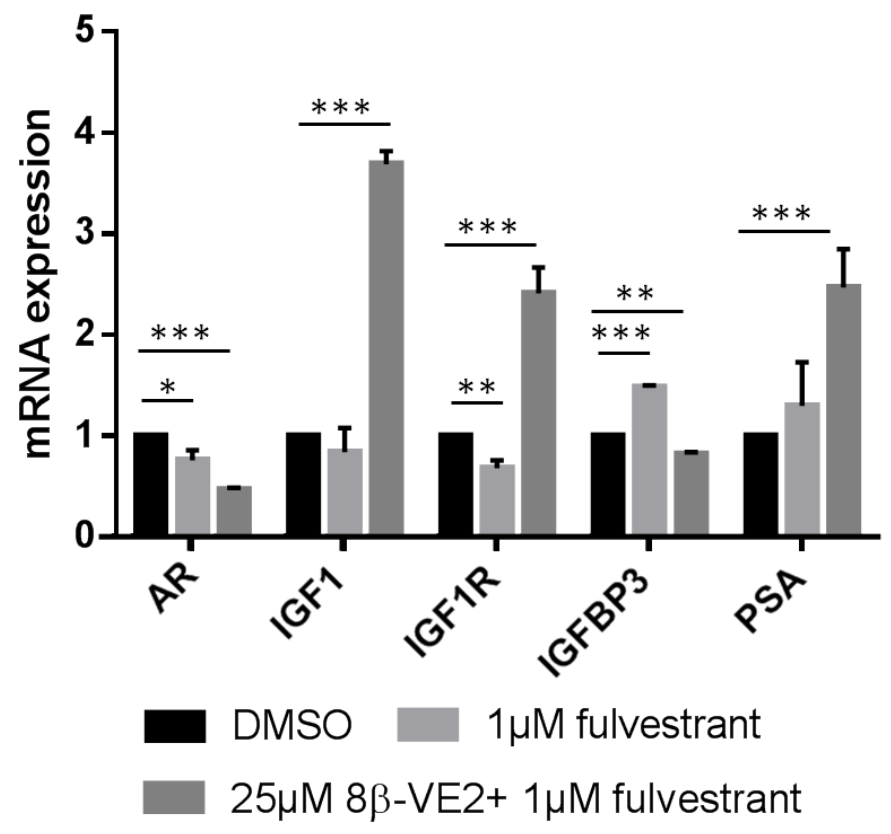

Figure 3.23: $A R$ and $A R$ target gene expression in VCaP cells after $8 \beta-\mathrm{VE} 2$ and fulvestrant treatment. VCaP cells were treated with the ER $\beta$ agonist $8 \beta$-VE2 and the ER $\beta$ antagonist fulvestrant to investigate if the $8 \beta$-VE2-induced effects are mediated by ER $\beta$. Simultaneous treatment with $8 \beta$ VE2 and fulvestrant downregulated $A R$ expression and upregulated IGF1 and IGF1R expression comparable to single 8 $\beta$-VE2 treatment. IGFBP3 expression was downregulated in 8 $\beta$-VE2- and fulvestrant-treated VCaP cells. PSA expression was upregulated in $8 \beta-\mathrm{VE} 2+$ fulvestrant-treated cells. The data represent the mean \pm s.d. of two independent experiments, which were performed in duplicate. Significance was calculated using the student's t-test ${ }^{*} p<0.05,{ }^{* *} p<0.01,{ }^{* * *} p<0.0001$ compared to DMSO control.

\subsubsection{Analysis of the role of the cholesterol pathway in 8$\beta-V E 2-m e d i a t e d$ treatment effects}

To elucidate the signaling mechanism mediating the $8 \beta$-VE2-induced treatment effects on VCaP cell variants, different hypotheses based on the whole mRNA-sequencing analysis were investigated. The whole mRNA-sequencing analysis identified numerous genes connected to the cholesterol synthesis pathway to be upregulated after $8 \beta$-VE2 treatment (see table 3.2). 
Table 3.2: Differential expression of genes involved in the cholesterol synthesis pathway. Differentially expressed genes in 8 $\beta$-VE2-treated VCaP cells identified in whole mRNA-sequencing analysis. (+) Increased expression by $>\log 2$ fold $1,(-)$ unchanged expression

\begin{tabular}{|c|c|}
\hline Enzymes of cholesterol synthesis & $\begin{array}{l}\text { Expression in } 8 \beta \text {-VE2-treated VCaP } \\
\text { cells rel. to DMSO-treated VCaP cells }\end{array}$ \\
\hline ACAT2 & - \\
\hline HMGCS1 & + \\
\hline HMGCR & - \\
\hline MVK & + \\
\hline$P M V K$ & - \\
\hline$M V D$ & - \\
\hline IDI1/IDI2 & + \\
\hline FDPS & + \\
\hline GGPS1 & - \\
\hline FDFT1 & + \\
\hline SQLE & - \\
\hline LSS & - \\
\hline$D H C R$ & + \\
\hline
\end{tabular}

Cholesterol is the precursor of steroid hormones and therefore of major importance for androgen synthesis (Mostaghel 2013). Cholesterol synthesis is also known to be important in PCa progression (Montgomery et al. 2008). It was shown that the inhibition of cholesterol synthesis decreased proliferation, induced apoptosis and decreased cell migration and invasion of PCa cells (Murtola et al. 2012; Freeman, Solomon 2004). Furthermore, other ER $\beta$ agonists (e.g. HE3235) were claimed to inhibit the cholesterol synthesis by inhibiting the cholesterol side chain cleavage enzyme (P450scc) (Montgomery et al., 2010). Based on these previous results, VCaP cells were treated with the P450scc inhibitor aminoglutethimide (AGE) to test if inhibition of cholesterol metabolism is the underlying cause for cell reduction and apoptosis induction after $8 \beta$-VE2 treatment.

VCaP cells were treated with $5 \mu \mathrm{M}, 10 \mu \mathrm{M}, 20 \mu \mathrm{M}, 50 \mu \mathrm{M}$ and $100 \mu \mathrm{M}$ AGE for $24 \mathrm{~h}$. As control VCaP cells were treated with DMSO. Next, mRNA expression analyses of cholesterol 
synthesis enzymes and AR target genes were performed to test if similar effects as seen after $8 \beta$-VE2 treatment can be observed.

QRT-PCR analysis showed that neither the cholesterol synthesis enzymes IDI1, FDPS and $D H C R$ nor $A R$ nor AR target genes showed altered mRNA expression due to AGE treatment. Therefore, it is unlikely that inhibition of $\mathrm{P} 450 \mathrm{scc}$ represents the underlying mechanism of action of $8 \beta$-VE2 in VCaP cells (Fig. 3.24).

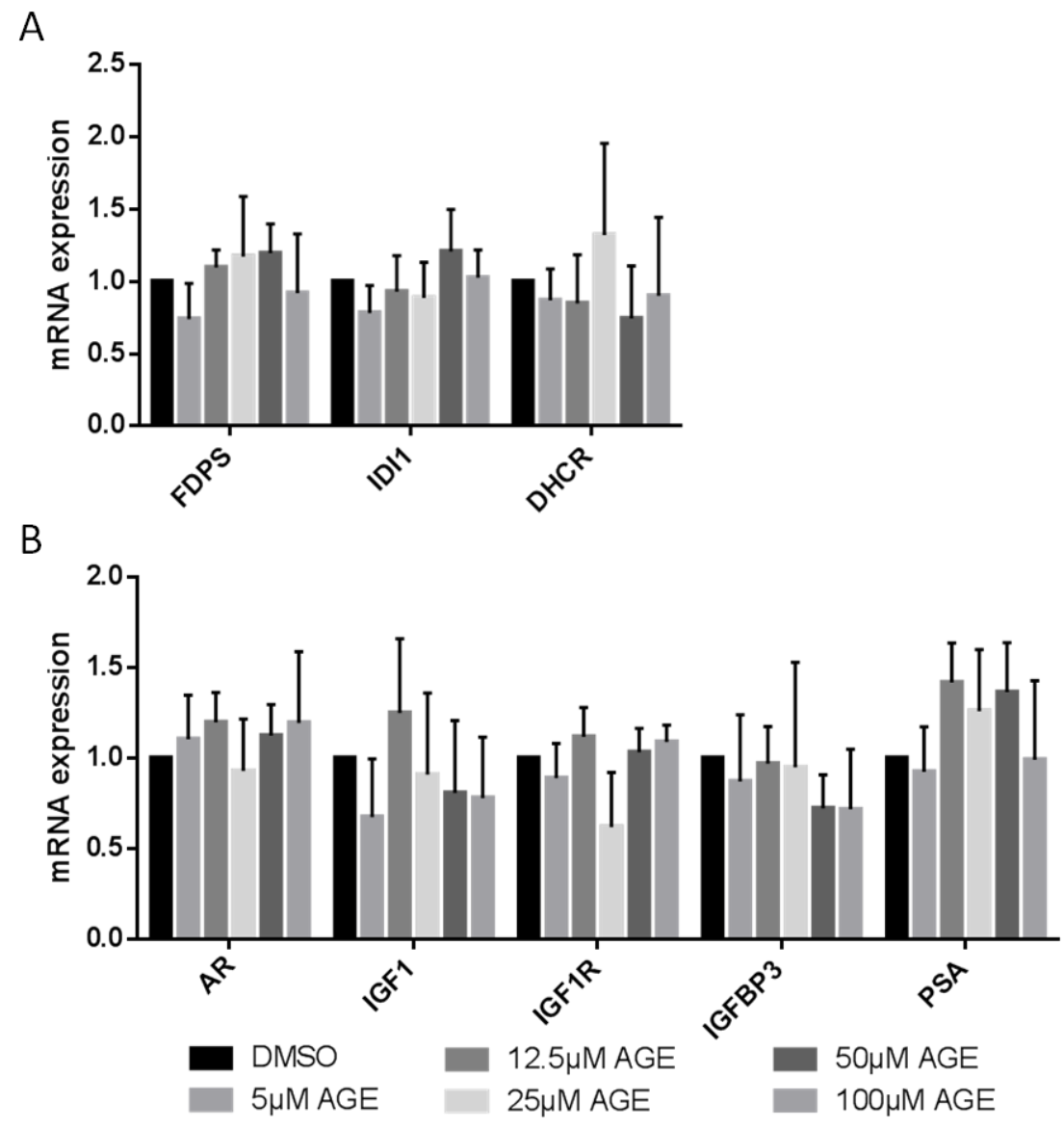

Figure 3.24: Effect of P450scc inhibition on VCaP cell gene expression involved in cholesterol synthesis and AR signaling. The expression of $(\mathbf{A})$ enzymes involved in cholesterol synthesis and (B) $A R$ and AR-regulated target genes was analyzed after treatment with the cholesterol side chain cleavage enzyme (P450scc) inhibitor AGE. No changes in gene expression of enzymes involved in cholesterol synthesis and $A R$ or AR target genes were observed. The data represent the mean \pm s.d. of two independent experiments, which were performed in duplicate. Significance was calculated using the student's t-test ${ }^{*} p<0.05,{ }^{* *} p<0.01,{ }^{* * *} p<0.0001$ compared to DMSO control.

\subsubsection{Analysis of the role of PI3K and MAPK signaling pathways in 8 $\beta$-VE2- mediated treatment effects}

The whole mRNA-sequencing analysis furthermore provided a link to PI3K involvement in $8 \beta$-VE2-induced effects in VCaP cell variants. PI3K regulators e.g. phosphoinositide-3-kinase interacting protein 1 were differentially expressed upon $8 \beta$-VE2 treatment. The PI3K signaling pathway is one of the major pathways involved in PCa development and 
progression (Bitting, Armstrong 2013). Kaarbo et al. (2010) described that inhibition of PI3K led to PCa regression. PI3K and AR signaling are connected via feedback inhibition (Qi et al. 2015). If $8 \beta-V E 2$ treatment leads to $P I 3 K$ inhibition, this could induce cell survival reduction and apoptosis induction. In addition, AR would no longer be inhibited by PI3K signaling. In this way, the observed upregulation of AR target genes upon $8 \beta$-VE2 treatment in VCaP cells could be explained.

\subsection{Analyses of PI3K and MAPK pathway inhibition as the underlying mechanism for $8 \beta$-VE2-induced effects on VCaP cells}

To analyze whether PI3K or MAPK inhibition is the underlying mechanism for the observed treatment effects of $8 \beta$-VE2, VCaP cells were starved with $0.3 \%$ dextran-coated charcoal stripped FBS for $24 \mathrm{~h}$ and treated with $25 \mu \mathrm{M} 8 \beta$-VE2 or DMSO as control for $24 \mathrm{~h}$. Then, cells were stimulated with $10 \%$ FBS for 10 min, whereas control cells were not stimulated. Proteins were isolated and immunoblotted with specific antibodies for the PI3K downstream target $\mathrm{pAKT/AKT}$ and MAPK downstream target pERK/ERK.

VCaP cells treated with $8 \beta$-VE2 showed only a weak phosphorylation of AKT, whereas cells treated with $8 \beta$-VE2 and FBS displayed increased AKT phosphorylation compared to FBStreated cells. Instead of inhibition of the PI3K pathway, 8$\beta$-VE2 treatment led to an increased activation of the PI3K downstream target AKT. No activation of ERK could be detected even in FBS-stimulated VCaP cells (Fig. 3.25). 


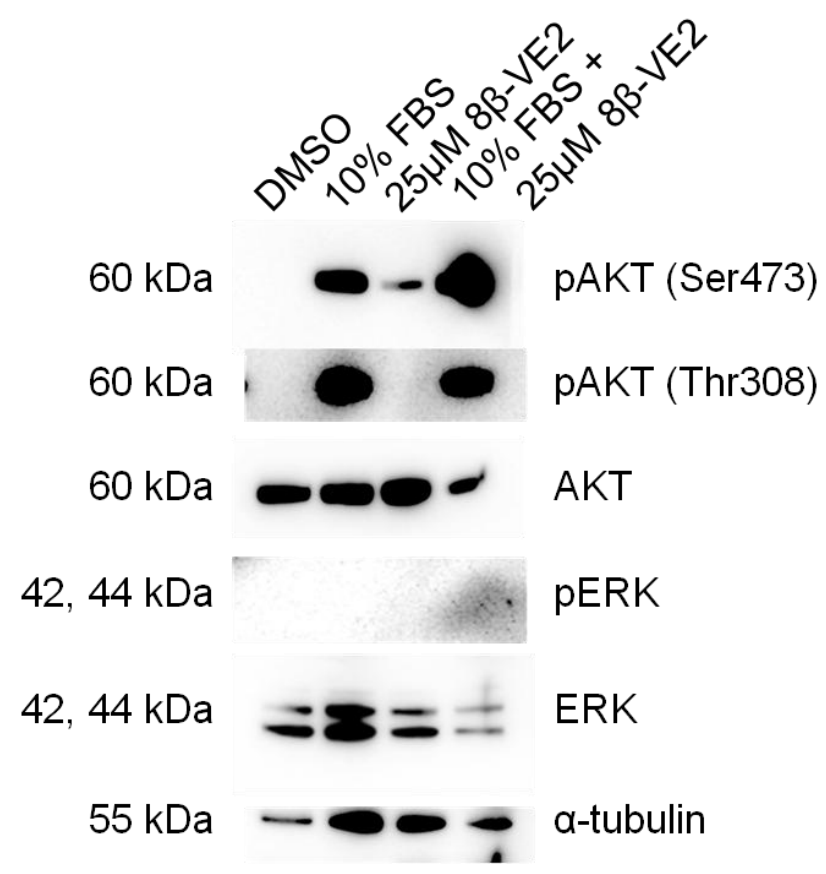

Figure 3.25: Phosphorylation of AKT and ERK in 8 $\beta$-VE2-treated VCaP cells. VCaP cells were starved for $24 \mathrm{~h}$, treated with $8 \beta$-VE2 for $24 \mathrm{~h}$ and stimulated with $10 \%$ FBS for 10 min. Protein was isolated and immunoblotted. Western blot analysis revealed that $8 \beta-V E 2$ treatment increased phosphorylation of AKT at Ser473. Stimulation of VCaP cells with FBS and 8 $\beta$-VE2 showed stronger phosphorylation of AKT than stimulation with FBS alone (upper panel). No phosphorylation of AKT Thr308 or ERK was detected (second and fourth panel). A representative western blot of two independent experiments, which were performed in duplicate is shown. $\alpha$-tubulin was used as a loading control (lower panel).

\subsection{Analysis of PI3K as a mediator of 8 $\beta$-VE2-induced effects in VCaP cells}

PI3K activation promotes cell survival and proliferation in PCa (Edlind, Hsieh 2014). We observed that $8 \beta$-VE2 treatment induced phosphorylation of the PI3K downstream target AKT. However, opposing effects would be expected if PI3K activation is the underlying cause for the effects observed by $8 \beta-V E 2$ treatment. To further clarify if the PI3K pathway is involved in the treatment effects observed upon $8 \beta$-VE2 treatment, VCaP cells were treated with both $8 \beta$-VE2 and the PI3K inhibitor GDC-0941 to analyze if 8 $\beta$-VE2-triggered effects still occur without PI3K signaling.

First, the concentration of the PI3K inhibitor GDC-0941 had to be determined, where AKT phosphorylation is reduced to a non-detectable level. For this purpose VCaP cells were cultured in $0.3 \%$ charcoal-stripped FBS and treated with $5 \mathrm{nM}, 50 \mathrm{nM}, 100 \mathrm{nM}, 1 \mu \mathrm{M}, 2.5 \mu \mathrm{M}$ and $5 \mu \mathrm{M}$ GDC-0941 for $2 \mathrm{~h}$. Next, VCaP cells were stimulated with $10 \%$ FBS for 10 min and proteins were isolated and immunoblotted with pAKT and AKT-specific antibodies. 
GDC-0941 concentrations $\geq 1 \mu \mathrm{M}$ completely inhibited the phosphorylation of AKT. The reduction in $\alpha$-tubulin expression showed that a concentration of $5 \mu \mathrm{M}$ GDC-0941 led to protein degradation (Fig. 3.26).

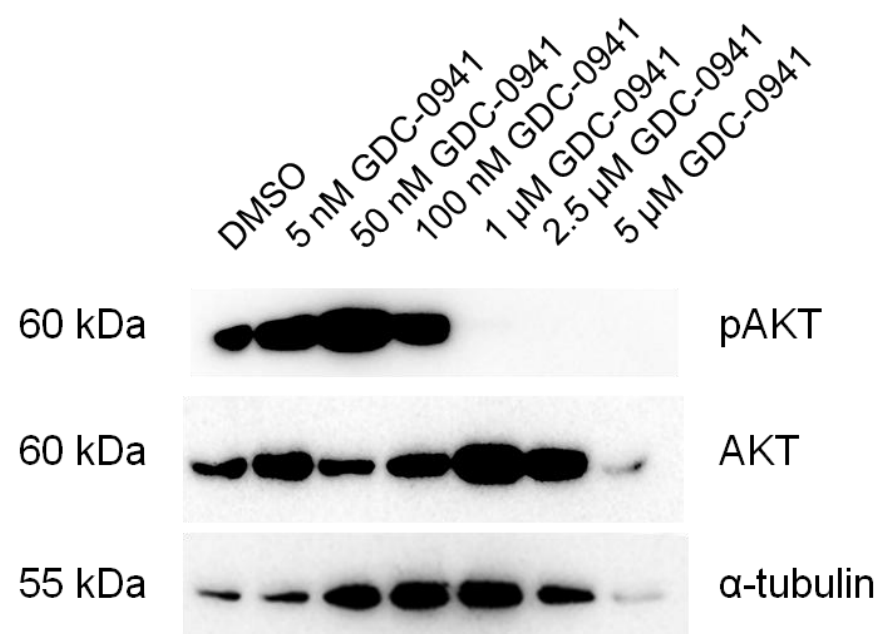

Figure 3.26: Phosphorylation of AKT after GDC-9041 treatment in VCaP cells. VCaP cells were treated with $5 \mathrm{nM}, 50 \mathrm{nM}, 100 \mathrm{nM}, 1 \mu \mathrm{M}, 2.5 \mu \mathrm{M}$ or $5 \mu \mathrm{M}$ of the PI3K inhibitor GDC-9041 and DMSO as control for $2 \mathrm{~h}$. Cells were stimulated with $10 \%$ FBS for $10 \mathrm{~min}$. Proteins were isolated and phosphorylation of AKT was detected as an indicator for PI3K activity. At a concentration of $\geq 1 \mu \mathrm{M}$ GDC-9041 phosphorylation of AKT was completely inhibited in VCaP cells (upper panel). A representative western blot is shown. The data represent one experiment, which was performed in duplicate. $\alpha$-tubulin was used as a loading control (lower panel).

Second, to examine if PI3K signaling has an impact on 8 $\beta$-VE2-mediated effects such as $A R$ and AR-regulated gene expression, VCaP cells were treated with $8 \beta-\mathrm{VE} 2$ and the PI3K inhibitor GDC-0941 for $24 \mathrm{~h}$. Cells were treated with FBS as positive control and DMSO as negative control. Subsequently, total RNA was isolated and $A R$ as well as AR target gene expression was analyzed as a marker for $8 \beta$-VE2-induced effects using qRT-PCR.

$A R$ expression was downregulated by FBS, FBS+GDC-0941 and 8 $\beta-V E 2+G D C-0941$ treatment. Up to 14.2-fold increased expression of IGF1 was observed in 8 $\beta$-VE2+GDC-0941 treated cells. IGF1R expression increased in FBS+GDC-0941-treated cells up to 7.4-fold and 5-fold in 8 8 -VE2+GDC-0941-treated cells. PSA expression increased up to 3.9-fold in FBS+GDC-0941 and 2.6-fold in 8 $\beta$-VE2+GDC-0941-treated cells. IGFBP3 expression increased upon FBS+GDC-0941 treatment by 2.8-fold and decreased in 8ß-VE2+GDC-0941treated cells by $47 \%$ (Fig. 3.27). In summary, PI3K inhibition could not inhibit the 8$\beta$-VE2mediated activation of the $A R$ and AR-regulated gene expression. Therefore, the observed effects upon $8 \beta$-VE2 treatment of VCaP cells are presumably not PI3K mediated. 


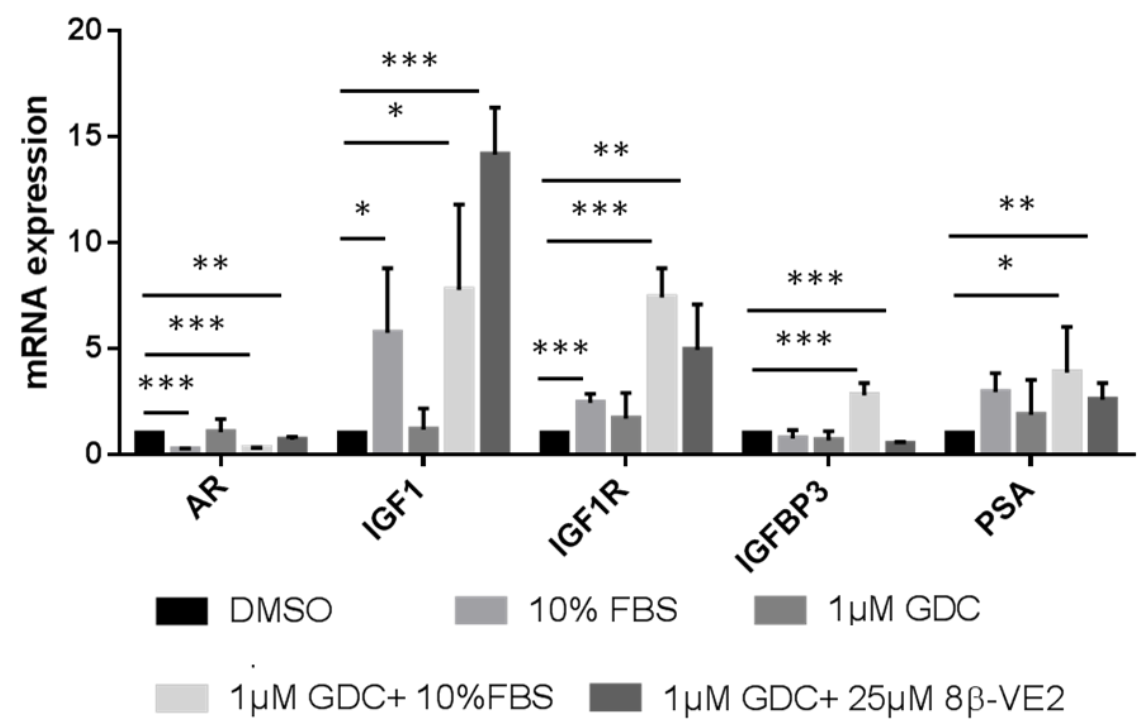

Figure 3.27: $\boldsymbol{A R}$ and AR target gene expression in VCaP cells after PI3K inhibition. VCaP cells were treated with $1 \mu \mathrm{M}$ of the PI3K inhibitor GDC-0941 and $10 \%$ FBS or $25 \mu \mathrm{M} 8 \beta$-VE2 for $24 \mathrm{~h}$. As control, VCaP cells were treated with DMSO, $10 \%$ FBS or $1 \mu \mathrm{M}$ GDC-0941. mRNA expression was analyzed using qRT-PCR. PI3K inhibition could not block the downregulation of the $A R$ or the expression of AR target genes induced by FBS or $8 \beta$-VE2 treatment. The data represent the mean \pm s.d. of two independent experiments, which were performed in duplicate. Significance was calculated using the student's t-test ${ }^{*} p<0.05,{ }^{* *} p<0.01,{ }^{* * *} p<0.0001$ compared to DMSO control.

\subsubsection{Investigation of underlying mechanism of AR target gene expression after 8 $\beta$-VE2 treatment}

As described above, AR target genes such as IGF1, IGF1R and PSA are overexpressed and the AR-repressed gene IGFPB3 is downregulated after $8 \beta$-VE2 treatment. Furthermore, whole mRNA-sequencing analyses showed that expression profiles of $8 \beta$-VE2-treated and DHT-treated VCaP cells showed significant concurrence (compared to Waltering et al. (2009), Fig. 3.28 and Tab. 3.3). 


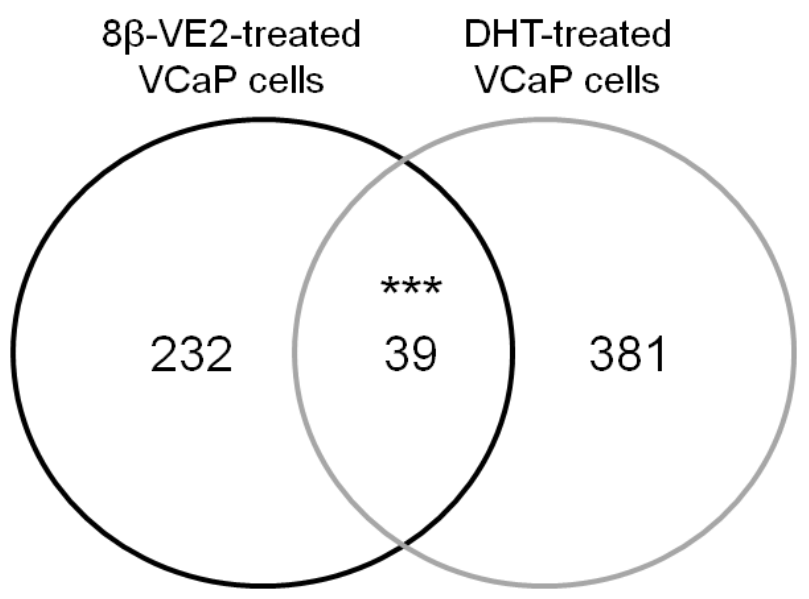

Figure 3.28: Expression similarities between 8$\beta$-VE2-treated and DHT-treated VCaP cells. Depicted is the overlap of differential gene expression in $8 \beta$-VE2-treated and DHT-treated VCaP cells. Differentially expressed gene in $8 \beta$-VE2-treated VCaP cells identified in the whole RNA-sequencing analyses were compared to genes differentially expressed in $\mathrm{VCaP}$ cells upon DHT treatment identified by Waltering et al. (2009). The concurrence of gene profiles is highly significant. The representation factor and the associated probability were calculated. ${ }^{*} p<0.05$, ${ }^{* *} p<0.01$, ${ }^{* * *}$ $\mathrm{p}<0.0001$

Table 3.3: Comparison of expression profiles of 8 $\beta$-VE2-treated VCaP cells and DHT-treated VCaP cells. Listed are the 10 most up- and downregulated genes regulated by $8 \beta$-VE2 and DHT treatment. The DHT-regulated genes were identified by Waltering et al. (2009).

\begin{tabular}{|c|c|c|c|}
\hline \multirow[b]{2}{*}{ Gene symbol } & \multirow[b]{2}{*}{ Gene name } & \multicolumn{2}{|c|}{ Log2 fold change } \\
\hline & & $\begin{array}{c}\text { DMSO vS. } \\
25 \mu M \text { 8ß-VE2 }\end{array}$ & $\begin{array}{l}\text { DMSO vs. } \\
\text { 10nM DHT }\end{array}$ \\
\hline AGR2 & anterior gradient 2 & 2.60 & 4.71 \\
\hline SLC2A3 & $\begin{array}{l}\text { solute carrier family } 2 \text { (facilitated } \\
\text { glucose transporter), member } 3\end{array}$ & 2.57 & 2.84 \\
\hline ST6GalNac1 & $\begin{array}{l}\text { ST6 (alpha-N-acetyl-neuraminyl- } \\
\text { 2,3-beta-galactosyl-1,3)-N- } \\
\text { acetylgalactosaminide alpha-2,6- } \\
\text { sialyltransferase } 1\end{array}$ & 1.98 & 2.78 \\
\hline SNAP91 & $\begin{array}{l}\text { synaptosomal-associated protein, } \\
91 \mathrm{kDa}\end{array}$ & 1.71 & 3.15 \\
\hline WIPI1 & $\begin{array}{l}\text { WD repeat domain, } \\
\text { phosphoinositide interacting } 1\end{array}$ & 1.69 & 3.56 \\
\hline FKBP5 & FK506 binding protein 5 & 1.63 & 6.60 \\
\hline NEFH & neurofilament, heavy polypeptide & 1.39 & 2.44 \\
\hline
\end{tabular}




\begin{tabular}{c|l|c|c}
\hline SMOC2 & $\begin{array}{l}\text { SPARC related modular calcium } \\
\text { binding 2 }\end{array}$ & 1.38 & 1.91 \\
\hline$I D I 1$ & $\begin{array}{l}\text { isopentenyl-diphosphate delta } \\
\text { isomerase 1 }\end{array}$ & 1.33 & 2.31 \\
\hline ODC1 & ornithine decarboxylase 1 & 1.14 & 3.44 \\
\hline$T T R$ & transthyretin & -1.07 & -1.43 \\
\hline$E F N B 2$ & ephrin-B2 & -1.27 & -2.00 \\
\hline$M G P$ & matrix Gla protein & -1.36 & -2.18 \\
\hline$R O R C$ & RAR-related orphan receptor C & -1.36 & -1.10 \\
\hline THBS1 & thrombospondin 1 & -1.43 & -2.94 \\
\hline SYTL2 & dopa decarboxylase & -1.52 & -2.47 \\
\hline$A R$ & synaptotagmin-like 2 & -1.54 & -2.40 \\
\hline$A S Z 1$ & androgen receptor & -1.72 & -1.00 \\
\hline$E R P 27$ & leucine zipper domain containing 1 & -2.83 & -1.64 \\
\hline & endoplasmic reticulum protein 27 & -2.98 & -1.18 \\
\hline
\end{tabular}

With regard to the role of $\mathrm{AR}$ signaling in $\mathrm{PCa}$ different putative underlying mechanisms causing this effect were analyzed in subsequent experiments.

\subsection{Analysis of glucocorticoid receptor expression and transcriptional activity in VCaP cells}

Advanced PCa cells can employ the glucocorticoid receptor (GR) signaling to circumvent AR signaling. In those resistant PCa cell lines the GR is overexpressed and leads to expression of a subset of typical AR-regulated genes (Arora et al. 2013). To examine if this regulatory mechanism explains the induction of AR target gene expression after $8 \beta-V E 2$ treatment, the expression of GR and its target gene SGK1 were analyzed using qRT-PCR. VCaP rev., VCaP and VCaP AA cells were treated with $25 \mu \mathrm{M} 8 \beta$-VE2 or DMSO as control for $24 \mathrm{~h}$. Total RNA was isolated, cDNA generated and GR and SGK1 expression were quantified using gene-specific primer pairs.

The qRT-PCR analyses showed that $G R$ was not overexpressed in 8 $\beta$-VE2-treated cells compared to control cells. In contrast, in VCaP rev. cells the expression of $G R$ was downregulated by $62 \%$ after $8 \beta$-VE2 treatment. Only in VCaP cells the expression of SGK1 was downregulated by $33 \%$, VCaP rev. and VCaP AA cells showed no altered expression of 
SGK1 due to $8 \beta$-VE2 treatment (Fig. 3.29). This result indicates that the induction of $A R$ target gene expression is not induced by GR overexpression.

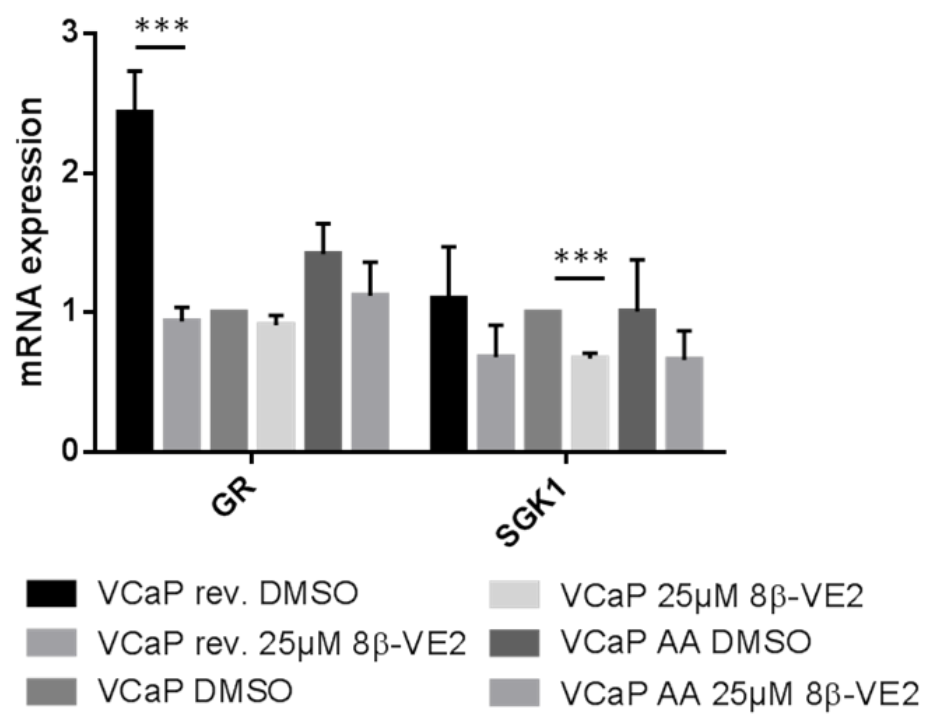

Figure 3.29: Expression of $G R$ and SGK1 in VCaP cells after $8 \beta-\mathrm{VE} 2$ treatment. VCaP rev., VCaP and VCaP AA cells were treated with $25 \mu \mathrm{M} 8 \beta$-VE2 or DMSO as control for $24 \mathrm{~h}$. GR and SGK1 mRNA expression was analyzed using qRT-PCR. GR expression was downregulated in VCaP rev. cells after $8 \beta$-VE2 treatment. SGK1 expression was downregulated in VCaP cells after $8 \beta-\mathrm{VE} 2$ treatment. The data represent the mean \pm s.d. of two independent experiments, which were performed in duplicate. Significance was calculated using the student's t-test. ${ }^{*} p<0.05,{ }^{* *} p<0.01,{ }^{* \star *} p<0.0001$ compared to DMSO control.

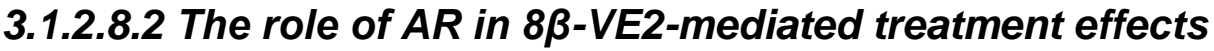

The induction of $A R$ target gene expression could also result from the activation of $A R$ signaling. Therefore, different approaches were made in order to elucidate the role of AR signaling in the observed effects after $8 \beta$-VE2 treatment in VCaP cell variants. To investigate if $A R$ activation could be the underlying reason for $8 \beta$-VE2-mediated treatment effects, the effects of AR activation in VCaP cells were studied. Furthermore, the effect of AR inhibition in combination with $8 \beta$-VE2 treatment should be analyzed. We examined if testosterone released by dying $\mathrm{VCaP}$ cell variants could be responsible for AR activation and performed a reporter gene assay to show if $A R$ is transcriptionally active after $8 \beta$-VE2 treatment.

\subsection{Analysis of $A R$ and $A R$ target gene expression after inhibition of testosterone or AR signaling in VCaP cell variants}

To investigate if the intratumoral synthesis of testosterone might induce AR activation, VCaP rev., $\mathrm{VCaP}$ and $\mathrm{VCaP}$ AA cells were treated simultaneously with $3.25 \mathrm{mg} / \mathrm{ml}$ testosteronebinding antibody ( $T$ antibody) or control immunoglobulin $\mathrm{G}(\lg \mathrm{G})$ and $8 \beta$-VE2 for $24 \mathrm{~h}$. RNA was isolated and $A R$ and $A R$ target gene expression was analyzed using qRT-PCR. 
In VCaP and VCaP AA cells downregulation of $A R$ expression by app. $50 \%$ and induction of AR target gene expression was observed after $8 \beta$-VE2 treatment with or without $T$ antibody treatment (Fig. 3.30 A, B). VCaP rev. cells showed 2-fold $A R$ upregulation and downregulation of AR target gene expression after $8 \beta$-VE2 treatment with or without testosterone antibody treatment (Fig. $3.30 \mathrm{C}$ ). Thus, testosterone release seems to have no impact on the observed AR signaling activation after $8 \beta$-VE2 treatment. 
A

VCaP rev.

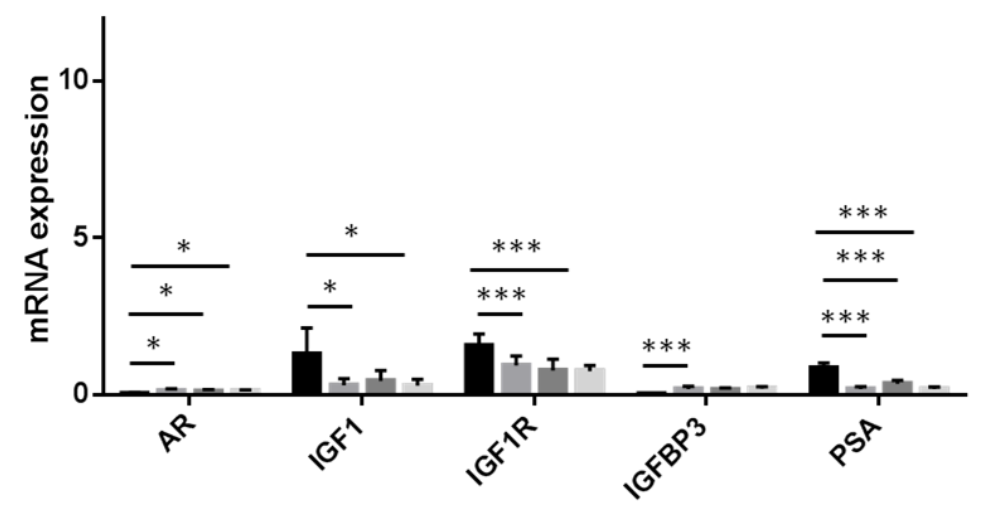

B

VCaP

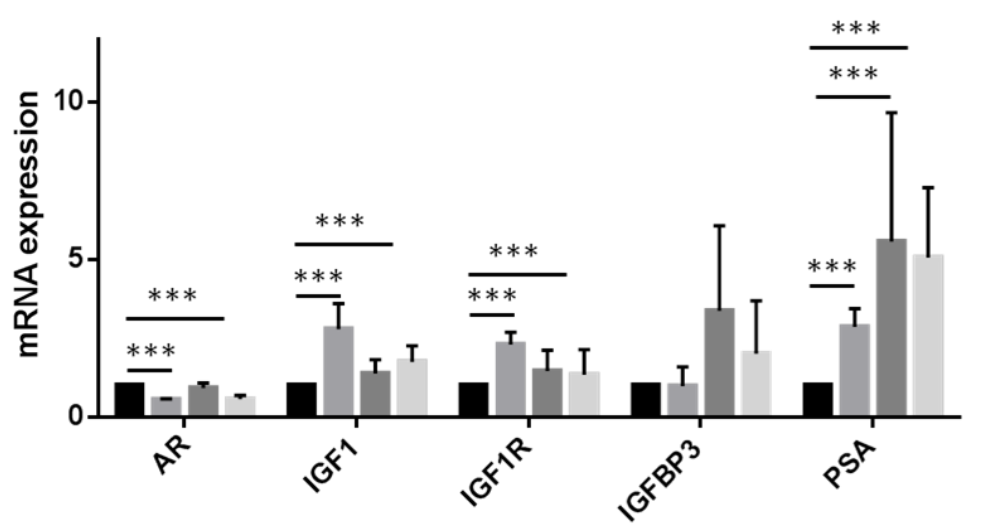

C

VCaP AA

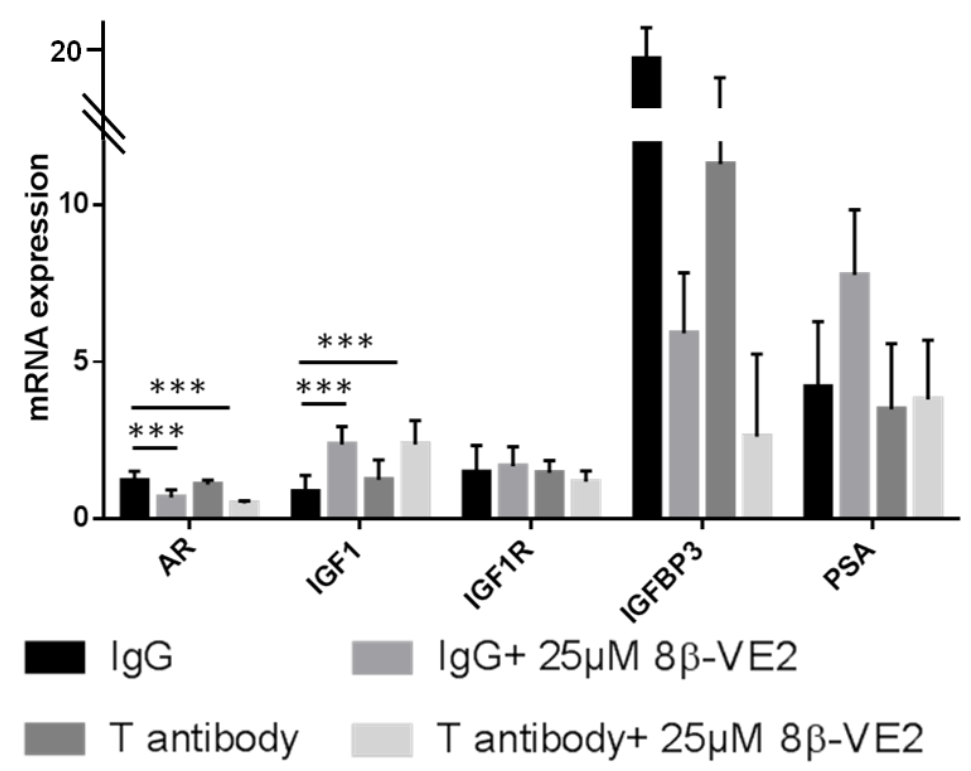

Figure 3.30: mRNA expression of $A R$ and $A R$ target genes after $8 \beta-V E 2$ and testosterone antibody treatment in VCaP cell variants. (A) VCaP rev., (B) VCaP and (C) VCaP AA cells were treated with $8 \beta$-VE2, testosterone-binding antibody ( $T$ antibody) or $8 \beta$-VE2 and $\mathrm{T}$ antibody for $24 \mathrm{~h}$. Immunoglobulin $\mathrm{G}$ ( $\mathrm{IgG}$ ) was used as control. The treatment of $\mathrm{VCaP}$ cell variants with the $\mathrm{T}$ antibody could not inhibit 8 $\beta$-VE2-mediated gene expression. The data represent the mean \pm s.d. of two independent experiments, which were performed in duplicate. Significance was calculated using the student's t-test ${ }^{*} p<0.05,{ }^{* *} p<0.01,{ }^{* * *} p<0.0001$ compared to IgG control. 
To further elucidate the role of $A R$ in $\mathrm{VCaP}$ cells, the effect of $A R$ inhibition on $8 \beta-V E 2$ treatment effects should be analyzed. Therefore, VCaP cells were treated with the antiandrogen bicalutamide. A pretrial experiment revealed that bicalutamide did not act as an antiandrogen in VCaP cells but induced the expression of the AR-regulated genes IGF1 by 2.6-fold, IGF1R by 2.3-fold and PSA by 2.9 -fold. Bicalutamide is therefore not a suitable component for AR inhibition in VCaP cells (Fig. 3.31).

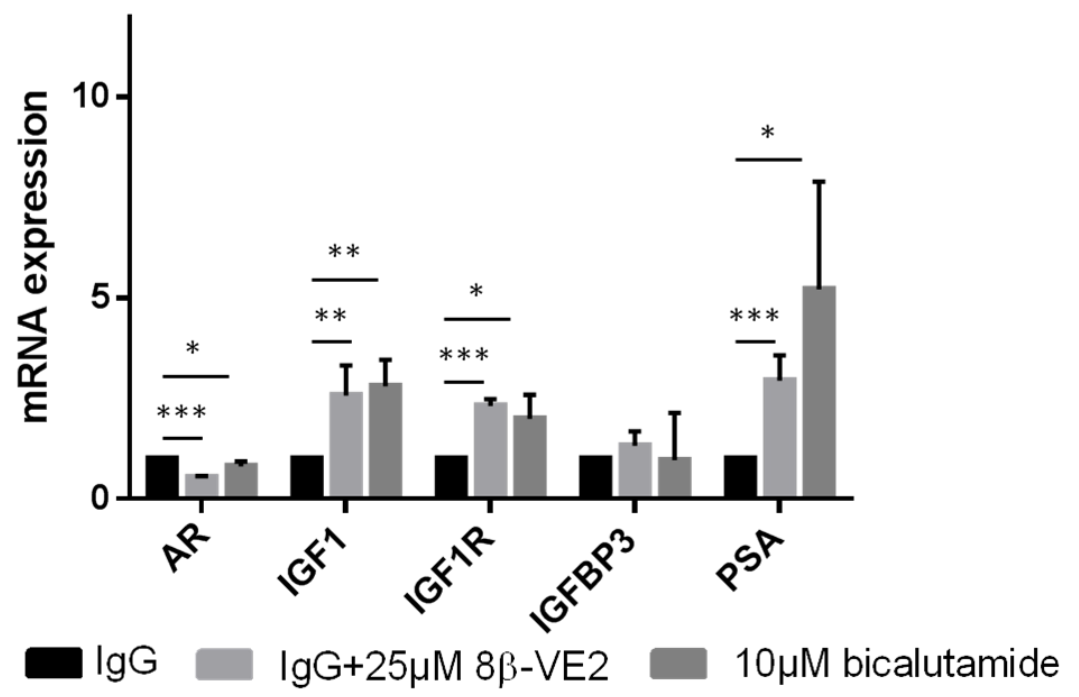

Figure 3.31: Expression of $A R$ and AR-regulated genes in bicalutamide-treated VCaP cells. VCaP cells were treated with IgG and $8 \beta-V E 2,10 \mu \mathrm{M}$ bicalutamide or IgG as control for $24 \mathrm{~h}$. qRTPCR was used to analyze the expression of $A R$ and $A R$ target gene expression. Although bicalutamide is an antiandrogen it induced AR-regulated gene expression. The data represent the mean \pm s.d. of two independent experiments, which were performed in duplicate. Significance was calculated using the student's t-test * $p<0.05,{ }^{* *} p<0.01$, ${ }^{* * *} p<0.0001$ compared to IgG control.

\subsection{Analysis of the effect of DHT treatment in VCaP cells}

Whole mRNA-sequencing analysis of $8 \beta$-VE2-treated VCaP cell variants showed differential expression of $A R$, AR-regulated genes and candidate genes identified. To elucidate if $A R$ activation mimics the effects observed upon $8 \beta$-VE2 treatment cell survival and apoptosis induction was analyzed after DHT treatment. VCaP cells were treated with $10 \mathrm{nM}, 100 \mathrm{nM}$ DHT or DMSO as control and cell viability and induction of apoptosis was measured after $24 \mathrm{~h}, 48 \mathrm{~h}$ and $72 \mathrm{~h}$. Furthermore, RNA was isolated after $24 \mathrm{~h}$. In order to compare DHTand $8 \beta$-VE2-regulated gene expression, qRT-PCR analyses were performed.

VCaP cell survival reduced after $24 \mathrm{~h}$ and $48 \mathrm{~h}$ treatment with $10 \mathrm{nM}$ or $100 \mathrm{nM}$ DHT up to $50 \%$ (Fig 3.32 A). However, apoptosis induction was not observed (Fig 3.32 B). 


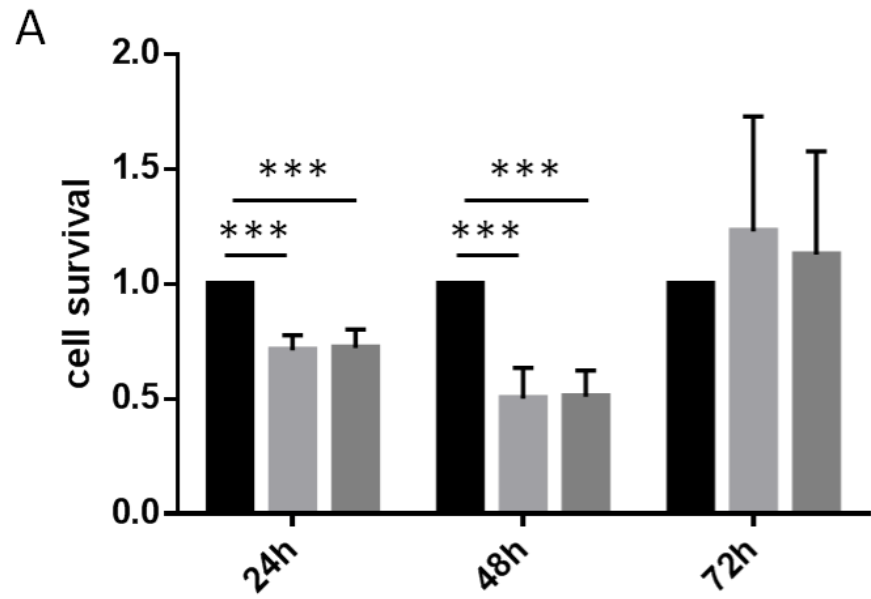

B

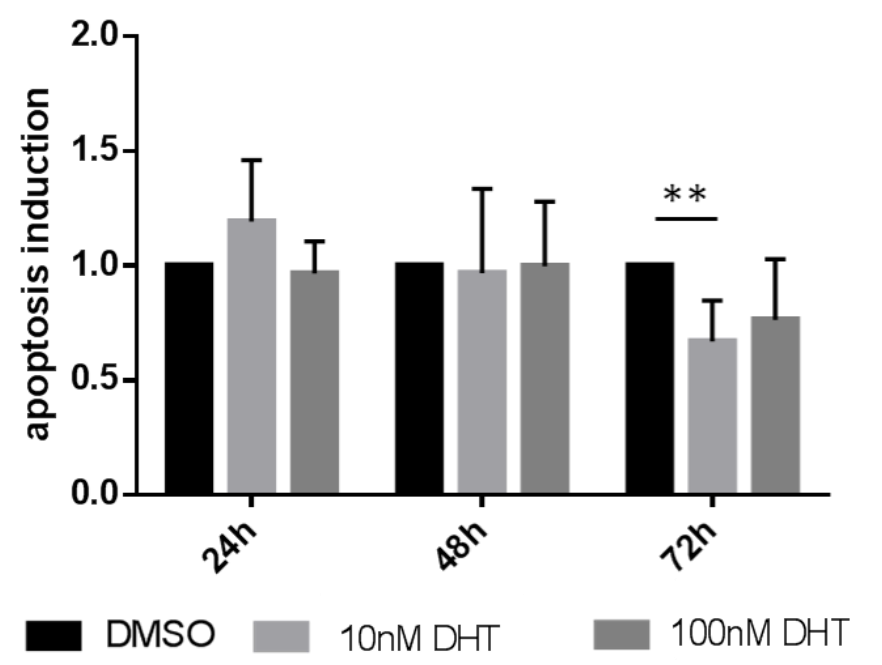

Figure 3.32: Cell survival and apoptosis induction in DHT-treated VCaP cells. VCaP cells were treated with $10 \mathrm{nM}, 100 \mathrm{nM}$ DHT or DMSO as control. (A) Cell survival and (B) apoptosis induction was analyzed after $24 \mathrm{~h}, 48 \mathrm{~h}$ and $72 \mathrm{~h}$. DHT-treated VCaP cells showed cell survival reduction up to $50 \%$ after $24 \mathrm{~h}$ and $48 \mathrm{~h}$, but apoptosis was not observed. The data represent the mean \pm s.d. of three independent experiments, which were performed in duplicate. Significance was calculated using the student's t-test. ${ }^{*} p<0.05,{ }^{* *} p<0.01,{ }^{* * *} p<0.0001$ compared to DMSO control.

Expression analyses showed that DHT treatment of $\mathrm{VCaP}$ cells induced similar changes in the analyzed genes as observed after $8 \beta$-VE2 treatment. $A R$ expression was downregulated by $94 \%$. AR-induced target gene expression was upregulated (IGF1 by 22-fold, IGF1R by 3.9-fold and PSA by 4.6-fold), whereas the negatively regulated AR target gene IGFBP3 was downregulated by $93 \%$. Expression of genes identified in the whole mRNA-sequencing analysis were either downregulated (WNT2 by $99 \%$, SHH by $89 \%$; MYB by $81 \%$, STAT6 by $75 \%$; PTGER2 by $86 \%$ ) or upregulated (SPOCK by 6 -fold, EAF2 by 11.7 -fold) after DHT treatment in a similar manner as it was shown after $8 \beta-V E 2$ treatment (see 3.1.2.3). Only FGFR1 expression did not change upon DHT treatment (Fig. 3.33). The data indicate that $\mathrm{DHT}$ and $8 \beta$-VE2 treatment induced similar effects on gene expression in VCaP cells. 


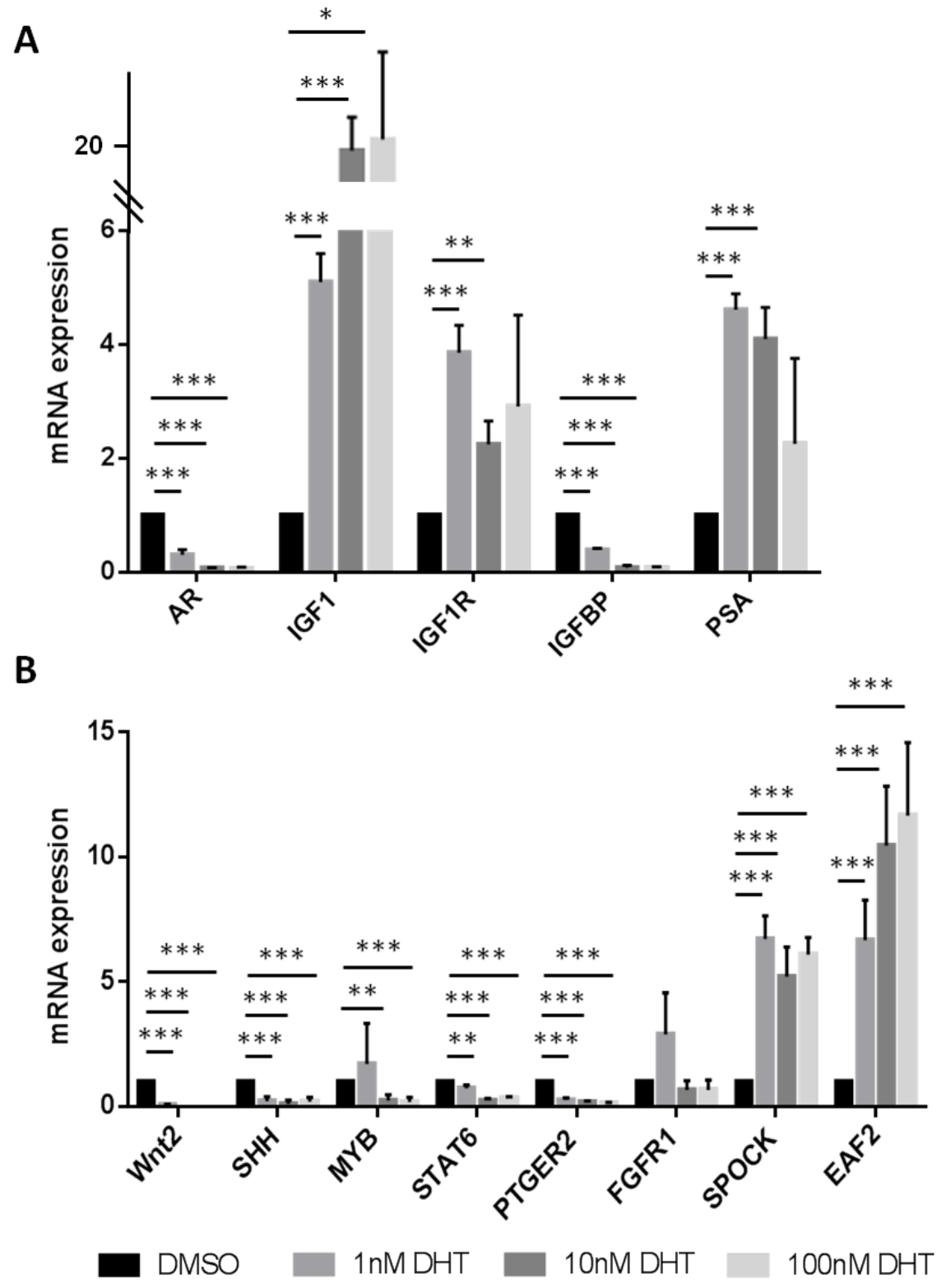

Figure 3.33: mRNA expression of $A R$, AR-regulated genes and whole mRNA-sequencing candidate genes in DHT-treated VCaP cells. VCaP cells were treated with $1 \mathrm{nM}, 10 \mathrm{nM}$ or $100 \mathrm{nM}$ DHT or DMSO as control for $24 \mathrm{~h}$. qRT-PCR was performed to quantify the expression of (A) $A R$ and its target genes and $(\mathbf{B})$ other whole mRNA-sequencing candidate genes. Already low concentrations of $1 \mathrm{nM}$ DHT led to a strong regulation of $A R$ and $A R$ target gene expression. Whole mRNAsequencing candidate genes showed a similar regulation pattern for expression as observed in $8 \beta-$ VE2-treated VCaP cells. Only the expression of the FGFR1 gene was not regulated by DHT. The data represent the mean \pm s.d. of two independent experiments, which were performed in duplicate. Significance was calculated using the student's t-test. ${ }^{*} p<0.05$, ${ }^{* *} p<0.01,{ }^{* * *} p<0.0001$ compared to DMSO control.

\subsection{Analysis of the effect of 8 $\beta$-VE2 on the human PCa cell line PC3}

In contrast to VCaP cells which display a high AR expression, the human PCa cell line PC3 does not express the AR (Kaighn et al. 1979). To further elucidate the role of AR signaling in 
$8 \beta$-VE2-mediated treatment effects, PC3 cells were treated with $25 \mu \mathrm{M} 8 \beta$-VE2 and DMSO as control for $24 \mathrm{~h}, 48 \mathrm{~h}$ and $72 \mathrm{~h}$ and cell survival and apoptosis induction were measured. The treatment of PC3 cells with $8 \beta$-VE2 did neither reduce cell survival (Fig. 3.34 A) nor induce apoptosis (Fig. 3.34 B).

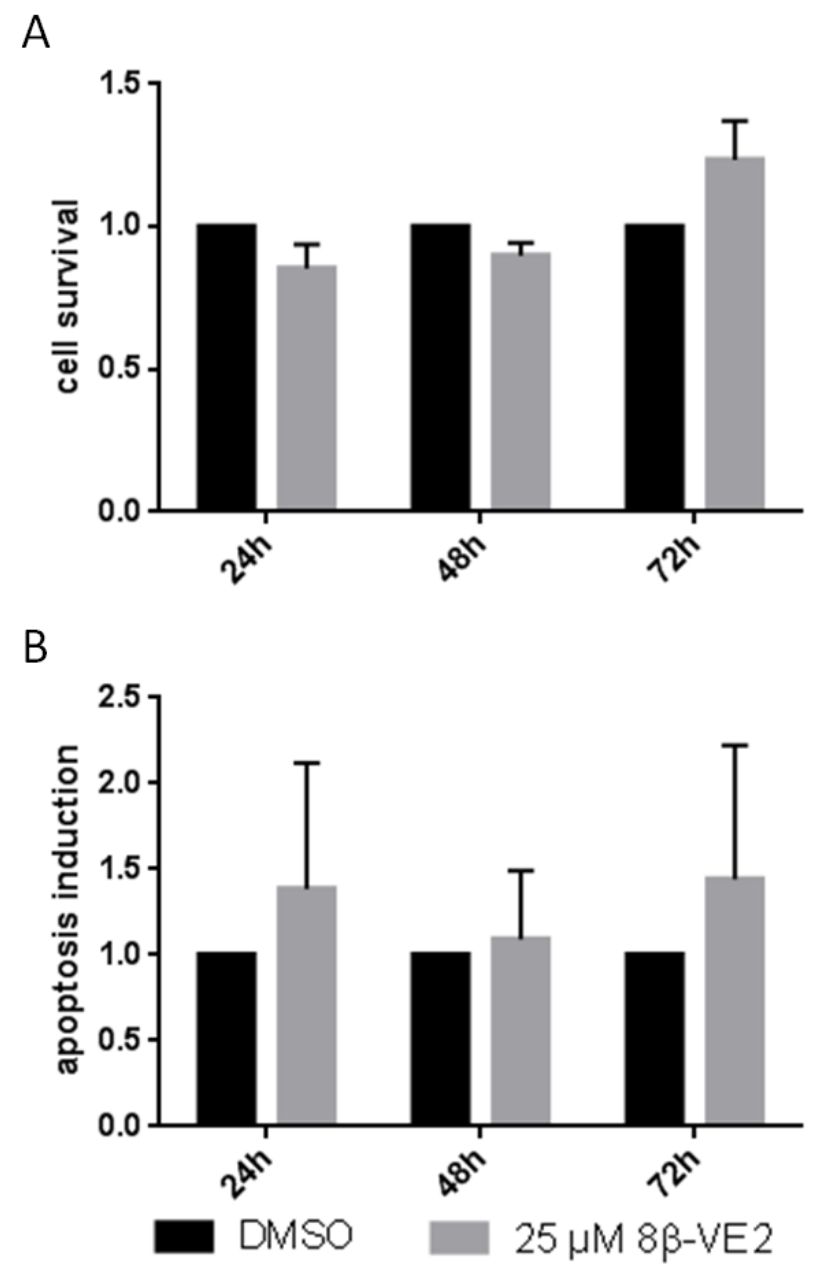

Figure 3.34: Cell survival and apoptosis induction in 8 $\beta$-VE2-treated PC3 cells. PC3 cells were treated with $25 \mu \mathrm{M} 8 \beta$-VE2 or DMSO as control. (A) Cell survival and (B) apoptosis induction was analyzed after $24 \mathrm{~h}, 48 \mathrm{~h}$ and $72 \mathrm{~h}$. After $8 \beta$-VE2 treatment of PC3 cells cell survival reduction or induction of apoptosis was not observed. The data represent the mean \pm s.d. of three independent experiments, which were performed in duplicate. Significance was calculated using the student's t-test. ${ }^{*} p<0.05,{ }^{* *} p<0.01,{ }^{* * *} p<0.0001$ compared to DMSO control.

\subsection{Analysis of AR activation after 8 $\beta$-VE2 treatment using a reporter gene assay in VCaP cells}

To investigate if $8 \beta$-VE2 treatment induced ER or AR transcriptional activity, a reporter gene assay was performed. Therefore, VCaP cells were transfected with different reporter vectors. These vectors carried specific promoter regions for ER (vitellogenin gene), steroid receptors in general (MMTV gene) or AR (probasin gene) coupled to a reporter gene (luciferase). Relative to the activation of the specific transcription factor the reporter gene is expressed. 
Furthermore, it was investigated if $A R$ activation could be induced by $8 \beta-V E 2$, the $E R$ ligand E2 or the ER $\beta$ ligand ERB 041. The activation of the AR could be ER $\beta$-dependent in VCaP cells and thus could be inhibited by the ER $\beta$ antagonist fulvestrant. VCaP cells were transfected with the vitellogenin (vit-ERE-Luc), MMTV (MMTV-Luc) and probasin (rPb-Luc) reporter plasmid and treated with $10 \mathrm{nM} \mathrm{E2,} 10 \mathrm{nM}$ DHT as positive control and DMSO as negative control. Furthermore, VCaP cells were treated with $25 \mu \mathrm{M} 8 \beta$-VE2, $1 \mu \mathrm{M}$ fulvestrant, $8 \beta$-VE2 and fulvestrant, $25 \mu \mathrm{M}$ ERB 041 and a combination of ERB 041 and fulvestrant (see 2.21).

The vit-ERE-Luc reporter failed to give any signal even with E2 as positive control. DHT treatment led to a 15-fold induction of MMTV and probasin promoter-mediated luciferase expression. Treatment with $10 \mathrm{nM}$ E2 induced 2.1-fold increased expression of the MMTV reporter gene. Neither 8$\beta-$ VE2 nor ERB 041 induced MMTV reporter gene expression. 8 $\beta$ VE2 treatment induced a significant increase of probasin reporter gene expression, but the induction was very weak (1.4-fold) (Fig. 3.35). It is unlikely, that this marginal induction is the underlying cause of $8 \beta$-VE2-mediated activation of AR target genes. 


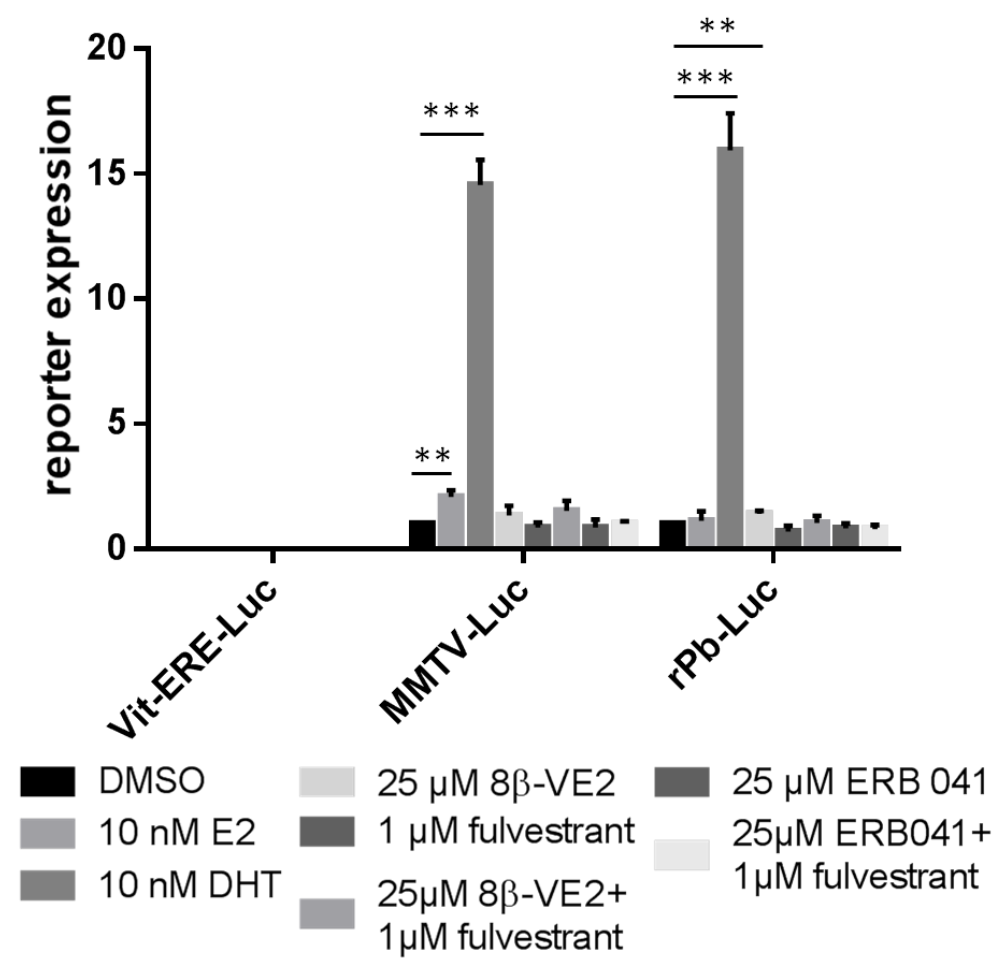

Figure 3.35: Transcriptional activity of AR and ER $\beta$ upon $8 \beta$-VE2 treatment in VCaP cells. VCaP cells, transfected with the reporter vectors Vit-ERE-Luc, MMTV-Luc or rPb-Luc were treated with

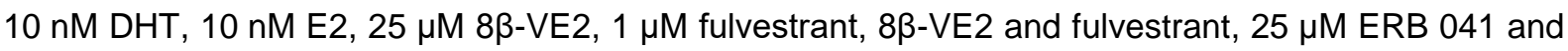
combination of ERB 041 and fulvestrant, respectively, or DMSO as control for $24 \mathrm{~h}$. Treatment of VCaP cells with the Vit-ERE-Luc reporter construct did not lead to any reporter gene expression. DHT treatment led to a 15-fold induction of both MMTV- and probasin-driven reporter gene expression. E2 treatment induced 2.1-fold increased expression of the MMTV reporter gene. Neither 8 $\beta$-VE2 nor ERB 041 treatment induced MMTV reporter gene expression. 8$\beta$-VE2 treatment induced a 1.4-fold increased probasin reporter gene expression. The data represent the mean \pm s.d. of two independent experiments, which were performed in duplicate. Significance was calculated using the student's t-test. ${ }^{*} p<0.05,{ }^{* *} p<0.01,{ }^{* * *} p<0.0001$ compared to DMSO control.

\subsection{Influence of amygdalin on mouse prostate cancer}

\subsubsection{Amygdalin treatment of TRAMP-FVB mice}

Amygdalin is considered to be an effective cancer treatment drug. Current in vitro studies by the working group of Prof. Dr. Roman Blaheta, University Medical Center, Frankfurt, support the anti-cancerous potential of amygdalin in PCa treatment (Makarevic et al. 2016; Makarevic et al. 2014), but valid in vivo studies about the efficacy of amygdalin are missing. In cooperation with Prof. Dr. Roman Blaheta, we aimed to elucidate the effect of amygdalin on PCa development and progression in the mouse PCa model TRAMP-FVB. To analyze the cancer-preventive potential of amygdalin, treatment of mice was started at the age of six weeks. For observation of the curative potential of amygdalin, treatment was started at 16 weeks of age when prostate cancer already starts to develop (see Fig. 2.5). Since no valid 
data were available about appropriate dosage for in vivo experiments, different amygdalin concentrations and purifications were tested. For this purpose $10 \mathrm{mg} / \mathrm{kg}, 100 \mathrm{mg} / \mathrm{kg}$ (SigmaAldrich) and $100 \mathrm{mg} / \mathrm{kg}$ of amygdalin with higher purity (CuraFaktur $\mathrm{GmbH}$ ) were injected intraperitoneally five times a week. Each treatment group contained $>10$ mice. Water was used as solvent and control. Side effects, such as weight loss, behavioral changes and abnormalities in organ histology were recorded. Mice were sacrificed when a PCa of $>1 \mathrm{~cm}^{3}$ was palpable, the burden of SGCa was visible and the general health status worsened (according to IACUC guidelines), respectively. After sacrificing the animal, the prostate tumor, testis, seminal gland (SG), lymph nodes, spleen, kidney, liver and lung were stored in formalin or stored at $-80^{\circ} \mathrm{C}$ for isolation of RNA and protein. To obtain further information about metabolism and mechanism of action of amygdalin, blood was extracted for measurement of cyanide and thiocyanate concentration.

\subsubsection{Analysis of side effects in TRAMP-FVB mice associated with amygdalin treatment}

TRAMP-FVB mice showed no side effects to i.p. administered amygdalin. Amygdalin-treated mice had no weight loss and no significant differences in weight gain compared to $0 \mathrm{mg} / \mathrm{kg}$ amygdalin-treated control mice ( $n=10$ for each group) (Fig. 3.36). 
A
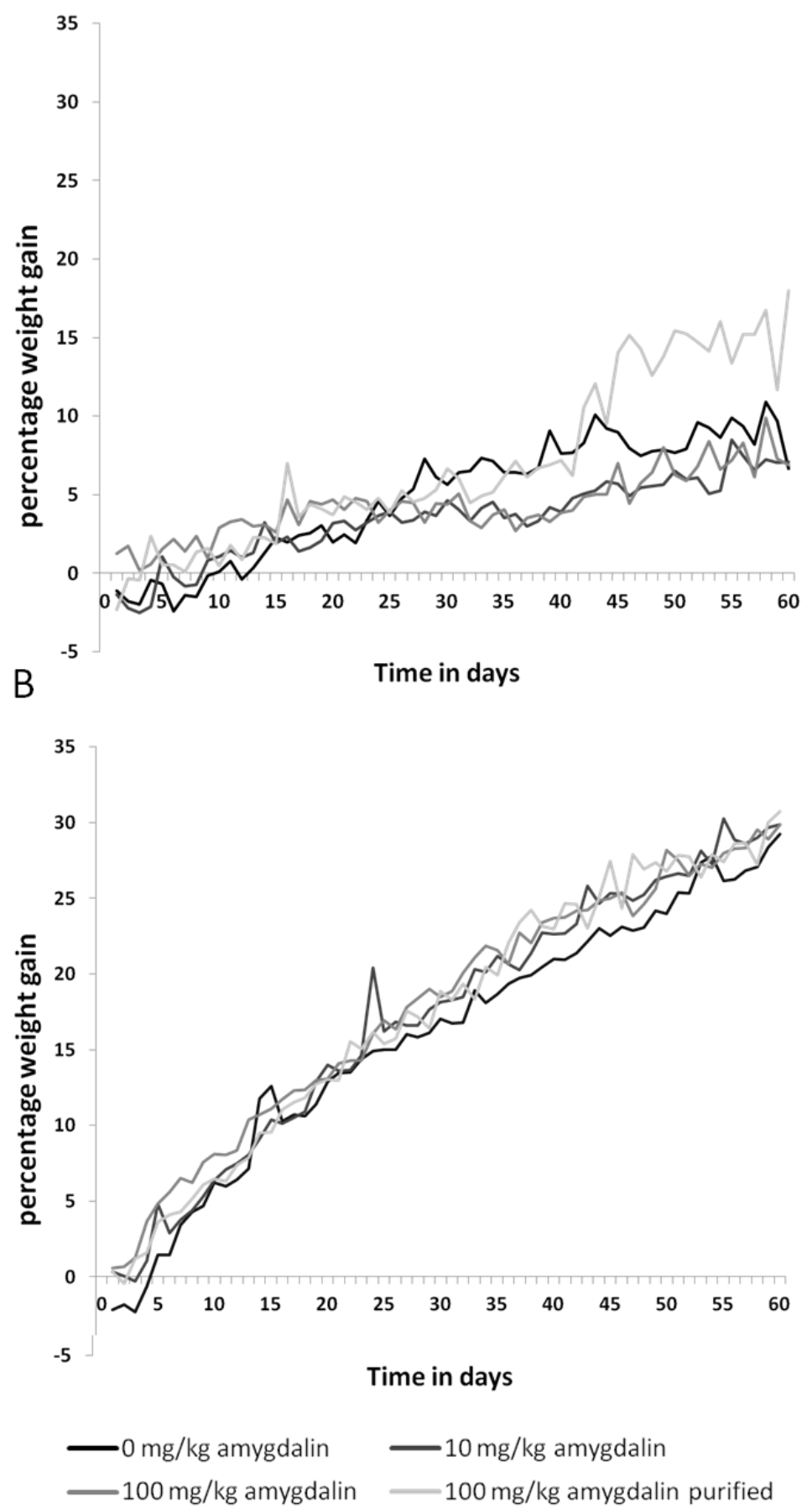

Figure 3.36: Weight gain of amygdalin-treated and control TRAMP-FVB mice. No significant differences in weight gain of $(\mathbf{A})$ curative or $(\mathbf{B})$ preventive amygdalin-treated and untreated mice could be detected. Depicted is the relative weight gain of $n=10$ mice during the first 60 days of treatment.

No behavioral abnormalities occurred in TRAMP-FVB mice. Furthermore, mice showed no signs of pain and the histopathological analysis showed no abnormalities in organ histology of liver, kidney, spleen or lung (untreated TRAMP-FVB mice $n=24$, amygdalin-treated TRAMP-FVB mice $n=18$ ) (Fig. 3.37 A). Moreover, histological PCa appearance, e.g. visible 
cell differentiation, was not affected by amygdalin treatment (Fig. 3.37 B). However, PCas of amygdalin-treated and control mice showed necrotic areas.

A
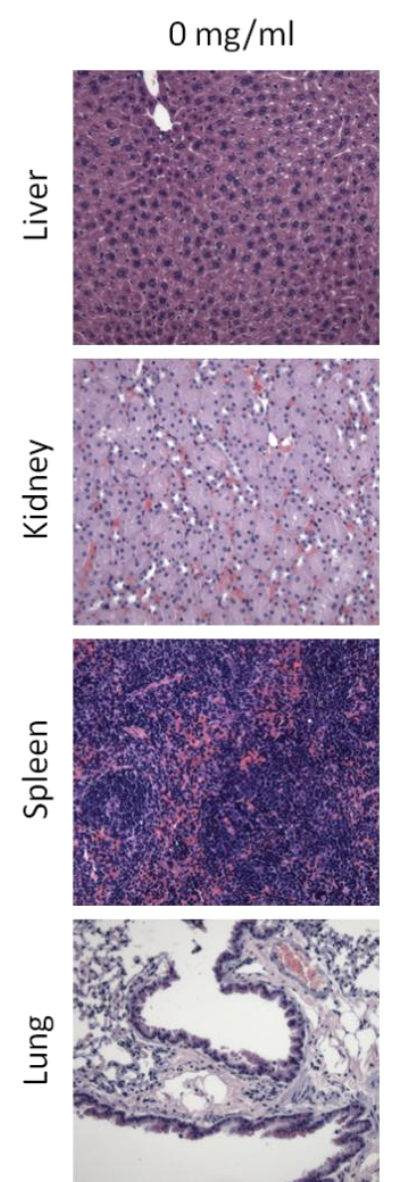

Amygdalin

$100 \mathrm{mg} / \mathrm{ml}$
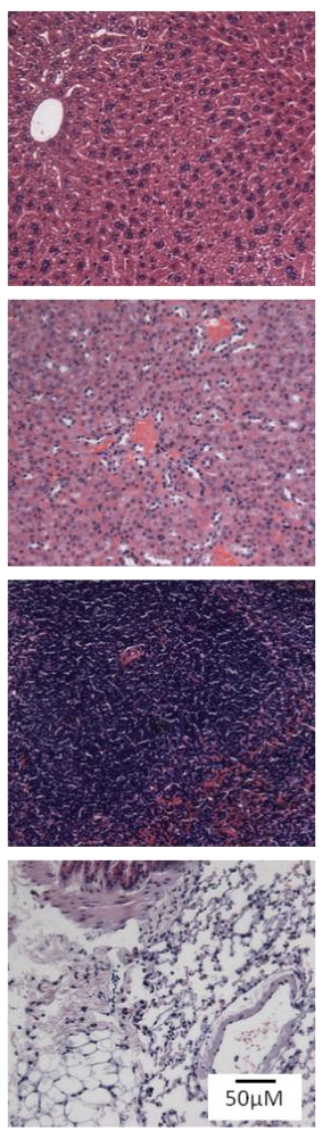

B

Amygdalin
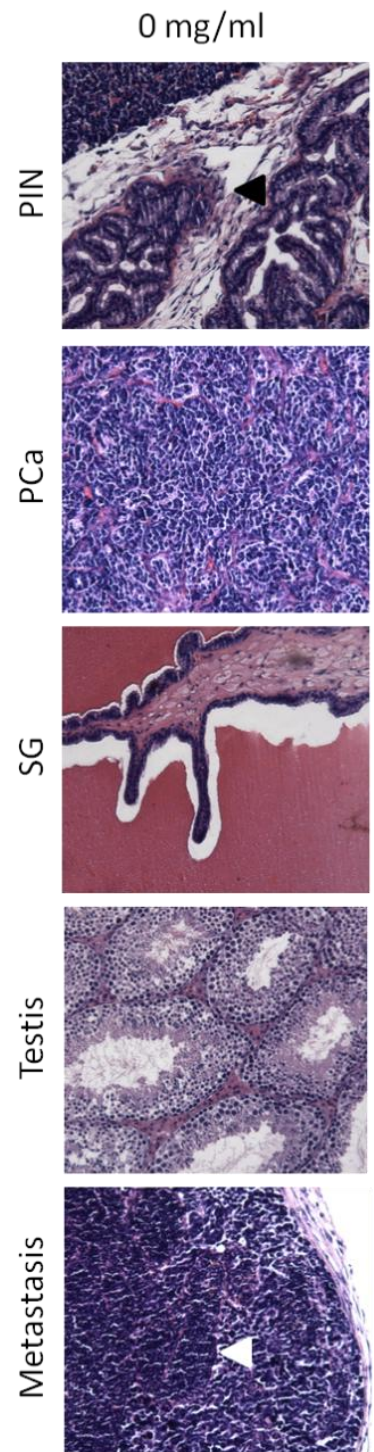
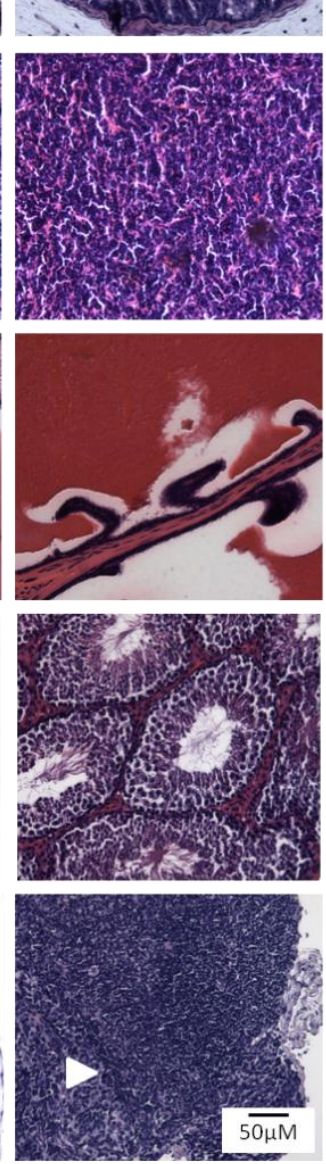

Figure 3.37: Hemalum and eosin stained tissue sections of amygdalin-treated and control TRAMP-FVB mice. (A) Toxic effects on liver-, kidney-, spleen- or lung-histology were not detected after amygdalin treatment. (B) Toxic effects on prostate-, seminal gland- (SG) or testis histology were also not observed. No histopathological differences in PIN, PCa or metastases occurrence, e.g. cell differentiation, were found in amygdalin-treated mice compared to control mice. PIN lesions are indicated by black arrow heads, metastasis by white arrow heads. Depicted are lymph node metastases. Representative tissue sections of $n=24$ untreated mice and $n=18$ amygdalin-treated mice are shown.

\subsubsection{Impact of amygdalin treatment on overall survival time of TRAMP-FVB mice}

Survival of treatment groups of TRAMP-FVB mice upon amygdalin treatment was recorded. Mice were sacrificed, when a PCa reached a tumor volume of $>1 \mathrm{~cm}^{3}$ or SGCa growth led to 
health impairment. Mice sacrificed for other reasons (e.g. injury due to the i.p. injections) were not included into the statistics.

Amygdalin treatment at any concentration showed no effect on the overall survival time of TRAMP-FVB mice in both curative and preventive treatment (Fig. 3.38).

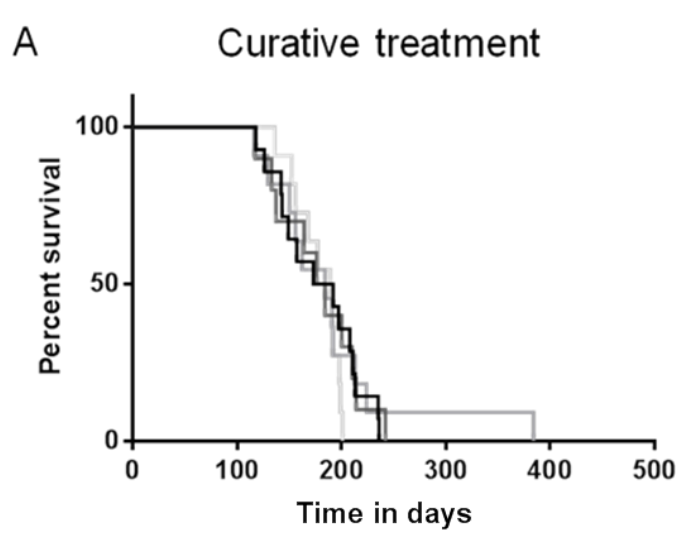

A

$\leftarrow 0 \mathrm{mg} / \mathrm{kg}$ amygdalin

$+10 \mathrm{mg} / \mathrm{kg}$ amygdalin $\mathrm{n}=9$

$+100 \mathrm{mg} / \mathrm{kg}$ amygdalin $\mathrm{n}=9$

$-100 \mathrm{mg} / \mathrm{kg}$ amygdalin $\mathrm{n}=8$ purified
B Preventive treatment

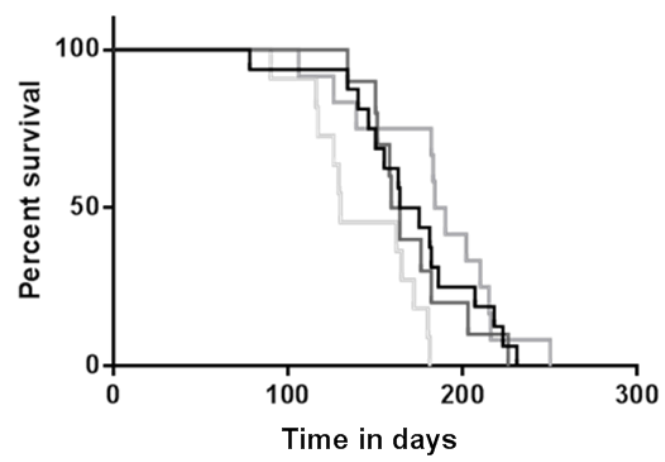

Curative Preventive

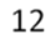

7

9

12

7

9

Figure 3.38: Survival study for amygdalin-treated and control TRAMP-FVB mice. Depicted is the median survival of the different treated mice in a Kaplan-Meier curve. Mice were treated with amygdalin or $\mathrm{H}_{2} \mathrm{O}$ as control starting from (A) 16 weeks (curative) or (B) six weeks (preventive) of age on five consecutive days per week. No significant changes between overall survival of the different cohorts were observed. Significance was calculated using the student's t-test.

\subsubsection{Tumor burden and metastasis formation in amygdalin-treated TRAMP- FVB mice}

Tumor stage and formation of metastasis were evaluated together with Dr. med. Felix Bremmer, Department of Pathology, University Medical Center, Göttingen. For the statistics, only the worst stage for each mouse was included, e.g. if one mouse showed PIN lesions, $\mathrm{PCa}$ and metastatic PCa, this mouse was counted to have metastatic PCa. Presence of SGCa was counted independently.

In the curative control treatment group approximately $14 \%$ of mice developed PIN lesions, $50 \%$ PCa and $36 \%$ developed metastatic PCa $(n=14) .36 \%$ of mice developed SGCa. In the curative $10 \mathrm{mg} / \mathrm{kg}$ amygdalin-treated group approximately $22 \%$ of mice developed PIN lesions, $33 \%$ PCa and $45 \%$ developed metastatic PCa $(n=9) .22 \%$ of mice developed SGCa. In the curative $100 \mathrm{mg} / \mathrm{kg}$ amygdalin-treated group approximately $20 \%$ of mice developed PIN lesions, $30 \%$ PCa and $50 \%$ developed metastatic PCa $(n=10) .60 \%$ of 
mice developed SGCa. In the curative $100 \mathrm{mg} / \mathrm{kg}$ purified amygdalin-treated group approximately $38.5 \%$ of mice developed PIN lesions, $38.5 \% \mathrm{PCa}$ and $23 \%$ developed metastatic PCa $(n=13) .46 \%$ of mice developed SGCa (Fig. 3.3 A). In the preventive control treatment group approximately $28 \%$ of mice developed PIN lesions, $39 \% \mathrm{PCa}$ and $33 \%$ developed metastatic PCa $(n=18) .17 \%$ of mice developed SGCa. In the preventive $10 \mathrm{mg} / \mathrm{kg}$ amygdalin-treated group approximately $10 \%$ of mice developed PIN lesions, $40 \%$ PCa and $50 \%$ developed metastatic PCa $(n=10) .20 \%$ of mice developed SGCa. In the preventive $100 \mathrm{mg} / \mathrm{kg}$ amygdalin-treated group approximately $46 \%$ of mice developed PIN lesions, $36 \%$ PCa and $18 \%$ developed metastatic PCa $(n=11) .64 \%$ of mice developed SGCa. In the preventive $100 \mathrm{mg} / \mathrm{kg}$ purified amygdalin-treated group approximately $27 \%$ of mice developed PIN lesions, $55 \%$ PCa and $18 \%$ developed metastatic PCa $(n=11) .36 \%$ of mice developed SGCa (Fig. 3.3 B). 
A

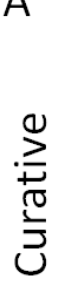

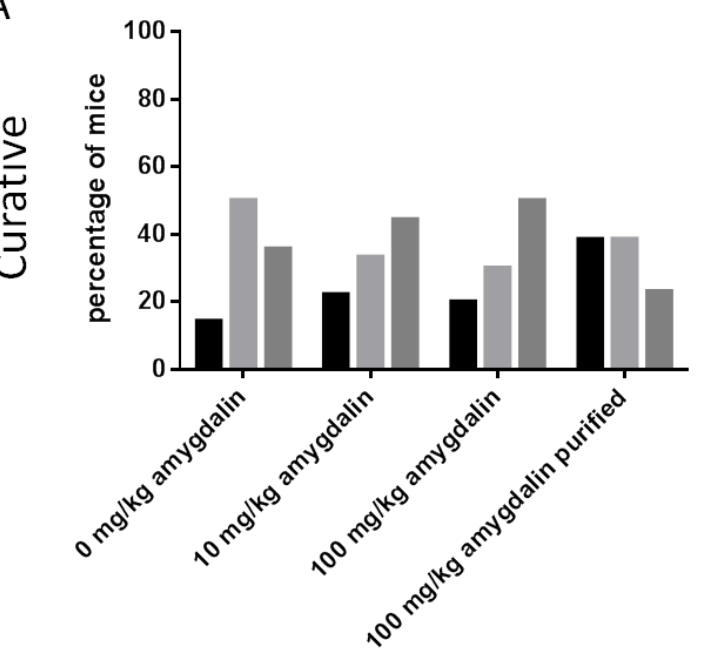

- $\mathrm{PIN} \mathrm{PCa} \quad$ metastatic $\mathrm{PCa}$

C

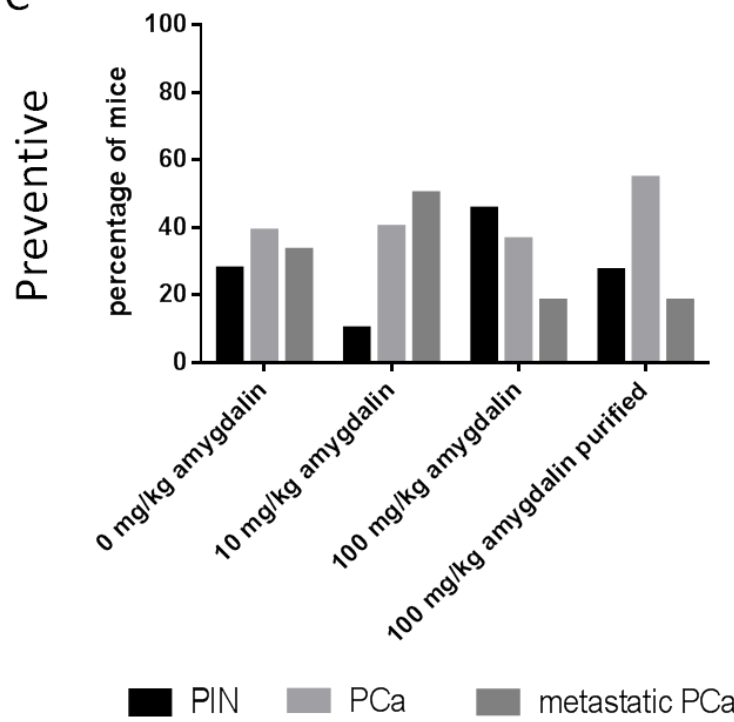

B

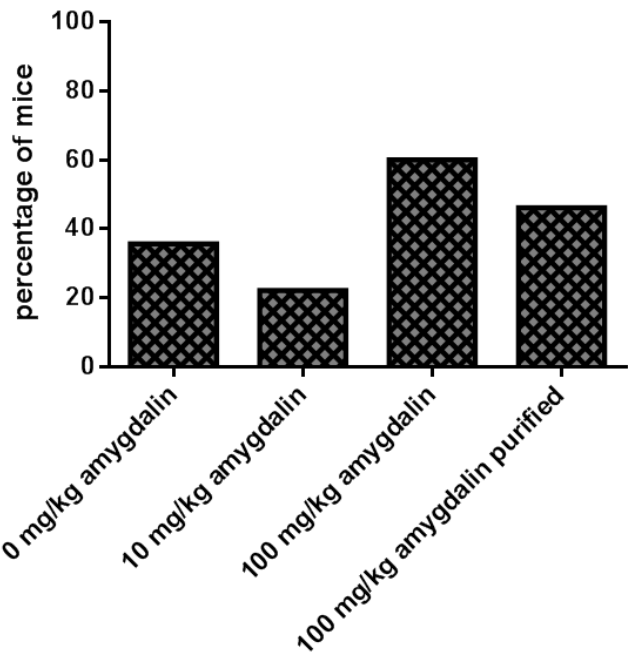

SGCa

D

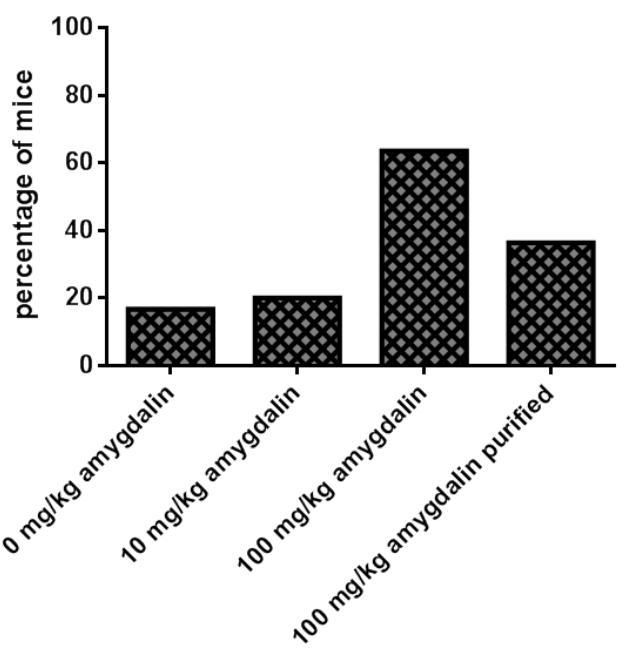

SGCa

Figure 3.39: Histopathological analysis of prostates and seminal glands of amygdalin-treated and control TRAMP/FVB mice. Depicted is the relative occurrence of cancer entities in each study group in percent. (A) In curative-treated mice no strong differences were observed in the incidence of tumor stages. Control-treated mice developed predominantly PCa (50\%) and metastatic PCa (36 \%). $10 \mathrm{mg} / \mathrm{kg}$ and $100 \mathrm{mg} / \mathrm{kg}$ amygdalin-treated mice developed predominantly metastatic PCa (45\% or $50 \%$ respectively). In $100 \mathrm{mg} / \mathrm{kg}$ purified amygdalin-treated mice predominantly PIN and PCa were observed $(38.5 \%)$ and mildly reduced incidence of metastatic PCa $(23 \%)$ was found. In the preventive treatment group a reduced incidence of metastatic PCa was found in $100 \mathrm{mg} / \mathrm{kg}$ amygdalinand purified amygdalin-treated mice. Control-treated mice developed predominantly PCa (39\%) and metastatic PCa (33\%). 10 mg/kg amygdalin-treated mice developed predominantly metastatic PCa (50\%). In $100 \mathrm{mg} / \mathrm{kg}$ amygdalin- and purified amygdalin-treated mice predominantly PIN and PCa were observed (100 mg/kg amygdalin $46 \%$ PIN and $36 \%$ PCa; $100 \mathrm{mg} / \mathrm{kg}$ purified amygdalin $27 \%$ PIN and $55 \%$ PCa) and reduced incidence of metastatic PCa (18\%) was found. (B) Increased occurrence of SGCa was observed in $100 \mathrm{mg} / \mathrm{kg}$ amygdalin- and purified amygdalin-treated mice compared to control or $10 \mathrm{mg} / \mathrm{kg}$ amygdalin-treated mice. Curative treatment: control $\mathrm{n}=14,10 \mathrm{mg} / \mathrm{kg}$ 
amygdalin $n=9,100 \mathrm{mg} / \mathrm{kg}$ amygdalin $\mathrm{n}=10,100 \mathrm{mg} / \mathrm{kg}$ purified amygdalin $\mathrm{n}=13$, preventive treatment: control $n=18,10 \mathrm{mg} / \mathrm{kg}$ amygdalin $n=10,100 \mathrm{mg} / \mathrm{kg}$ amygdalin $\mathrm{n}=10,100 \mathrm{mg} / \mathrm{kg}$ purified amygdalin $n=11$

\subsubsection{Examination of the mechanism of amygdalin metabolism}

It is still under debate how amygdalin could have effects on tumor cells. One possible explanation is the release of cyanide ions during metabolism of amygdalin (Fig. 1.4). These ions have cell toxic properties and induce cell death (Balkon 1982).

\subsection{Analysis of the expression of $\beta$-glucosidase and rhodanase in mouse tissues}

The enzyme $\beta$-glucosidase is considered to cleave amygdalin into its components glucose, cyanide and benzaldehyde (Haisman, Knight 1967). Therefore, higher $\beta$-glucosidase expression leads to more release of cell toxic cyanide. The enzyme rhodanase transfers sulfide groups to cyanide and therefore detoxicate the cyanide to thiocyanate. Hence, tissues with high $\beta$-glucosidase and low rhodanase expression should be very susceptible for amygdalin-induced cell death. Therefore, the expression pattern of both enzymes in different mouse tissues was analyzed using qRT-PCR. RNA was isolated from different tissues and differently treated mice. For each tissue and treatment group RNA of five TRAMP-FVB mice was isolated. For $\beta$-glucosidase the mouse $\beta$-glucosidase isoforms 1 and 2 were analyzed. All expression levels were calculated as change of expression to the expression level in the tumor tissue of the control-treated mouse \# 68 .

$\beta$-glucosidase 1 expression in tumor tissue was relatively low $(0.44$-fold $)$ compared to other tissues, e.g. spleen (7.9-fold). $\beta$-glucosidase 2 had similar expression levels in all tissues analyzed (between 0.27 -fold in kidney and 1.2-fold in liver). Rhodanase expression varied strongly between different tissues. Expression levels were high in kidney (54.9-fold), liver (360-fold) and lung (126-fold). Low expression of rhodanase was observed in tumor (0.87fold) and spleen (2.77-fold) (Fig. $3.40 \mathrm{~A})$. According to the hypothesized mechanism of action, strongest cyanide release should be observed in the spleen, followed by tumor tissue. Furthermore, it was analyzed if amygdalin treatment changes the expression of $\beta$ glucosidase or rhodanase in tumor or kidney tissue. All expression levels were calculated as change of expression to the expression level in the tumor tissue of control-treated mouse \# 72. No changes were observed in tumor or kidney tissue samples derived from TRAMPFVB mice treated with $10 \mathrm{mg} / \mathrm{ml}$ amygdalin compared to tissue samples from control-treated mice (Fig $3.40 \mathrm{~B}$ ). 
A

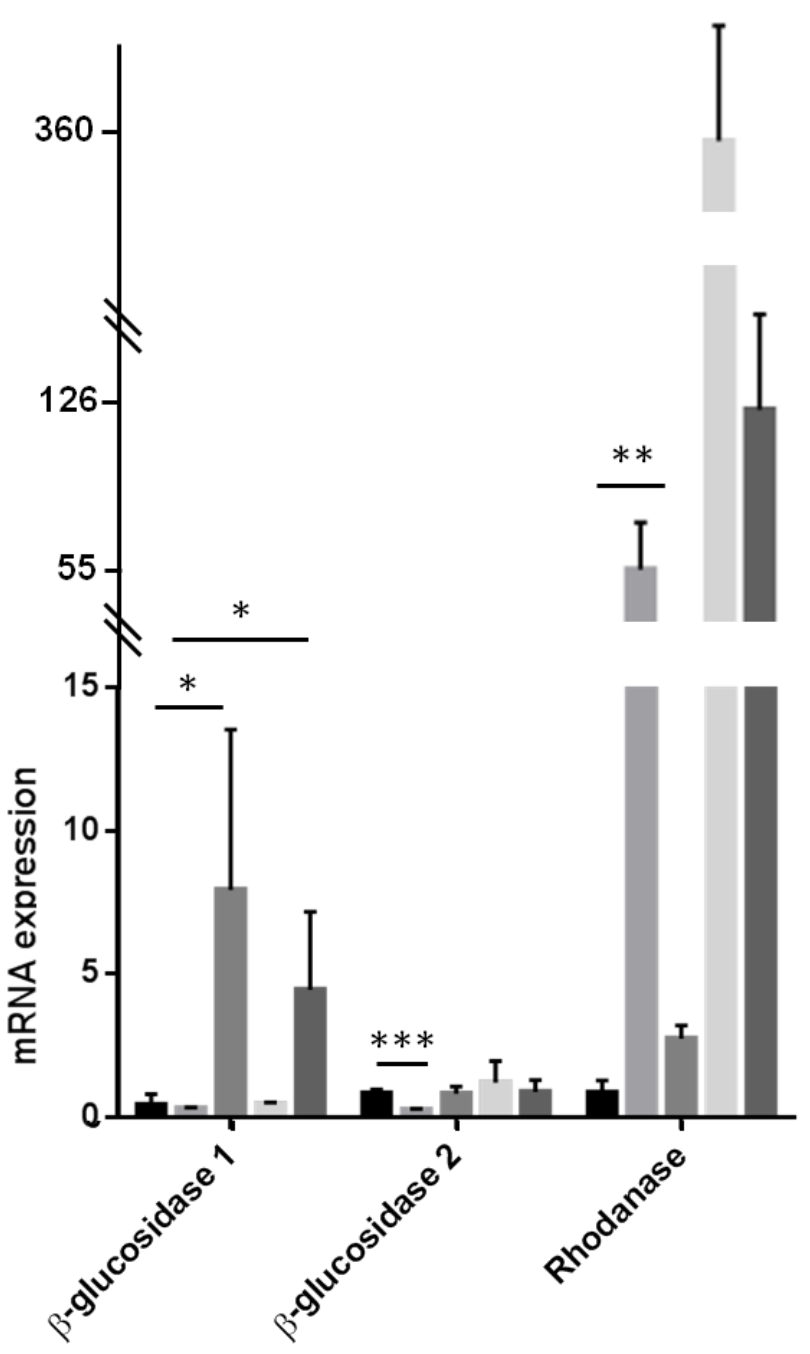

- Tumor $\square$ Kidney $\square$ Spleen $\square$ Liver $\square$ Lung 


\section{B}

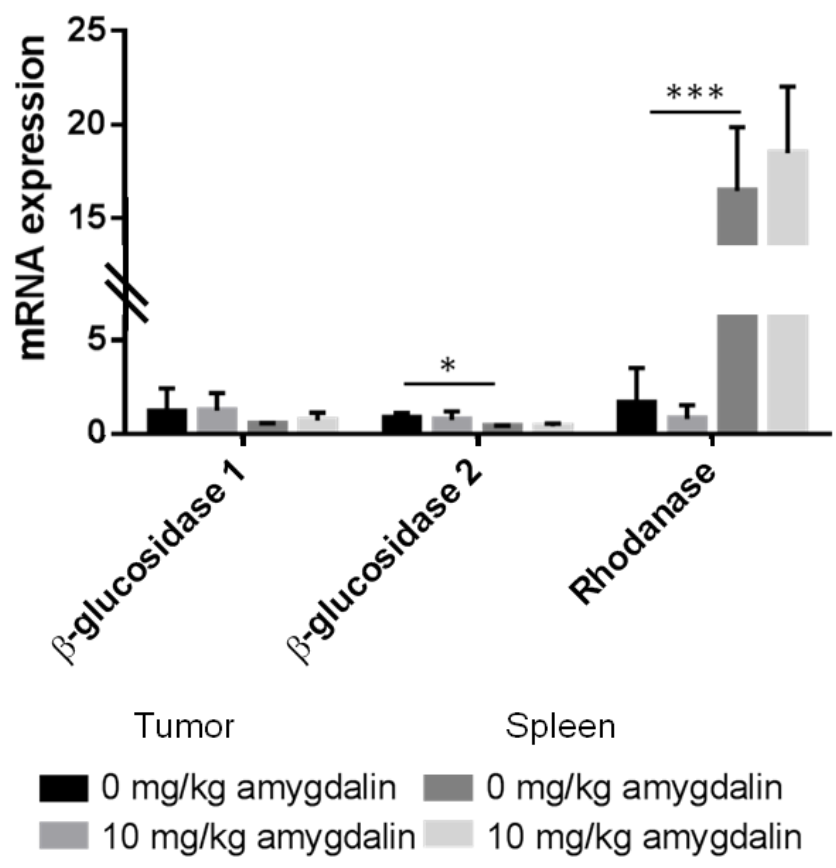

Figure 3.40: mRNA expression of $\beta$-glucosidase isoform 1 and 2 and rhodanase in tissues of TRAMP-FVB mice. (A) mRNA expression of $\beta$-glucosidase isoform 1 and 2 and rhodanase was analyzed in tumor, kidney, spleen, liver and lung of TRAMP-FVB mice $(n=5)$. $\beta$-glucosidase 1 expression was high in spleen and lung. $\beta$-glucosidase 2 expression was relatively low in all tissues analyzed. Rhodanase was weakly expressed in tumor and highly expressed in kidney, liver and lung. (B) Differential $\beta$-glucosidase and rhodanase expression in amygdalin-treated compared to control mice was analyzed. No expression changes induced by amygdalin treatment were observed. For data generation cDNA was generated twice and qRT-PCR was performed in two independent experiments. Depicted is the mean value \pm s.d. Significance was calculated using the student's t-test. ${ }^{*} p<0.05$, ** $p<0.01,{ }^{* * *} p<0.0001$ compared to tumor tissue of mouse \# 68 (A) and tumor tissue of mouse \# 72 (B).

\subsection{Detection of cyanide (CN) and thiocyanate (SCN) in blood samples of amygdalin-treated TRAMP-FVB mice}

Rauws et al. (1982) reported that i.p. injected amygdalin could be detected in blood of dogs 30 min after administration (Rauws et al. 1982). Here, we aimed to detect the metabolites $\mathrm{CN}^{-}$and $\mathrm{SCN}^{-}$in blood serum or EDTA blood. Mice were sacrificed 30 min after amygdalin administration. $\mathrm{SCN}^{-}$concentration in blood samples was analyzed by Dr. rer. nat. HansWolfgang Hoppe, head of the Umwelttoxikologie of the Medizinisches Labor Bremen. $\mathrm{CN}^{-}$ concentration in blood serum was analyzed by PD Dr. rer. nat. Michael Müller (Institute for Occupational, Social and Environmental Medicine of the University Medical Center, Göttingen) (see 2.25). 
No increase in $\mathrm{CN}^{-}$or in $\mathrm{SCN}^{-}$could be detected in serum (Fig. $3.41 \mathrm{~A}$ ) or blood samples (Fig. $3.41 \mathrm{~B}$ ) of amygdalin-treated TRAMP-FVB mice (10 or $100 \mathrm{mg} / \mathrm{kg}$ ) compared to control TRAMP-FVB mice $(n=5)$.

A

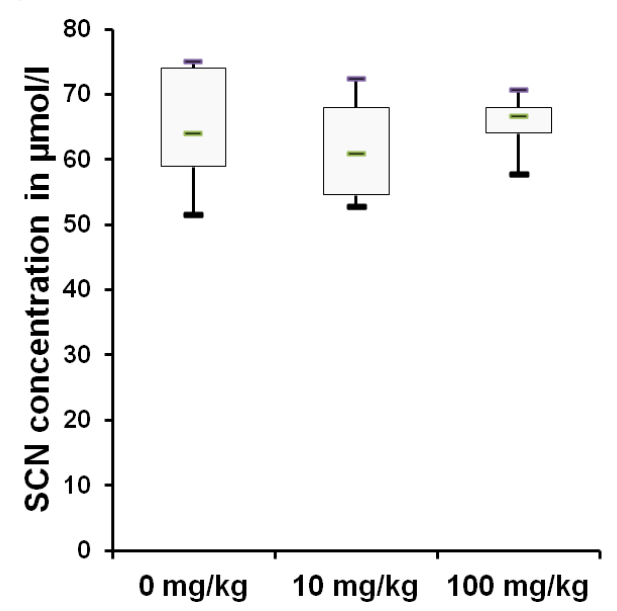

B

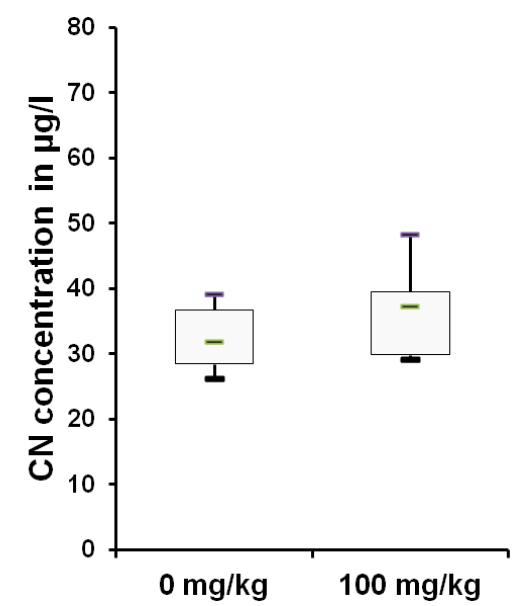

Figure 3.41: Concentration of $\mathrm{CN}^{-}$and $\mathrm{SCN}^{-}$in serum and blood of amygdalin-treated TRAMP-

FVB mice. Depicted is the median $\mathrm{SCN}^{-}$and $\mathrm{CN}^{-}$concentration in serum and blood of amygdalintreated mice in a box plot. Concentration of $(\mathbf{A}) \mathrm{CN}^{-}$and $(\mathbf{B}) \mathrm{SCN}^{-}$was measured in amygdalin-treated and control mice. No differences in $\mathrm{CN}^{-}$or $\mathrm{SCN}^{-}$concentrations were found between control and amygdalin-treated mice. $n=5$ mice for each group. Significance was calculated using the student's $t$ test.

\subsubsection{Correlation of amygdalin treatment and the formation of necrotic tumor areas in TRAMP-FVB mice}

The working group of Prof. Dr. Roman Blaheta was able to show in vitro that amygdalin reduces human $P C a$ cell number and induces a $G_{2}$-phase arrest (Makarevic et al. 2016). We hypothesized that cell number reduction might be associated with induction of apoptosis as shown for DU145 and LNCaP cells (Newmark et al. 1981; Chang et al. 2006). To examine if the in vitro results were applicable to the in vivo model, western blot and immunohistochemistry $(\mathrm{IHC})$ analyses of cell cycle-regulating and apoptosis-inducing genes should be performed. Therefore, protein was isolated from fresh frozen tissues and immunoblotted with specific antibodies.

Although samples were fresh frozen and different protein isolation techniques were tested, the quality of protein was not sufficient to produce valid results. With rising amygdalin concentration degradation of housekeeping proteins, e.g. HSC70 increased (Fig. 3.42). Therefore, western blot analysis was no appropriate analysis technique. 


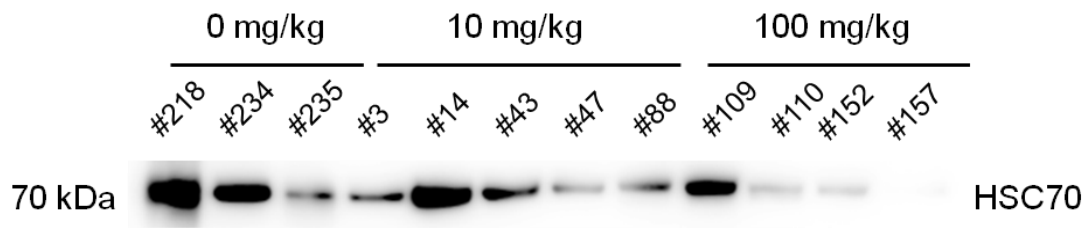

Figure 3.42: Protein integrity of amygdalin-treated PCa tumor samples. Protein was isolated from fresh frozen tissue samples. Protein concentration was measured using Bradford assay and $15 \mu \mathrm{g}$ protein were loaded for immunoblotting. Signal was detected using a HSC70-specific antibody. With increasing amygdalin concentrations the protein integrity worsened. A representative western blot of three experiments is shown.

To visualize the expression of cell cycle-regulating and apoptosis-inducing factors tumor tissue sections were stained using IHC. Tissues were harvested and fixed in $4 \%$ formalin immediately after scarifying the mice. Fixed tissue was embedded in paraffin and cut in $1 \mu \mathrm{m}$ thick slices. These slices were incubated with cleaved caspase 3 -specific antibodies to detect protein expression using a DAB chromogen based detection kit (see 2.22.2).

The massive occurrence of necrosis in tumor samples of TRAMP-FVB mice interfered with the detection of any signals (Fig. 3.43). Necrotic tissue is unspecifically stained, thus a definite conclusion about the expression of the tested proteins could not be drawn.

A Non-necrotic area

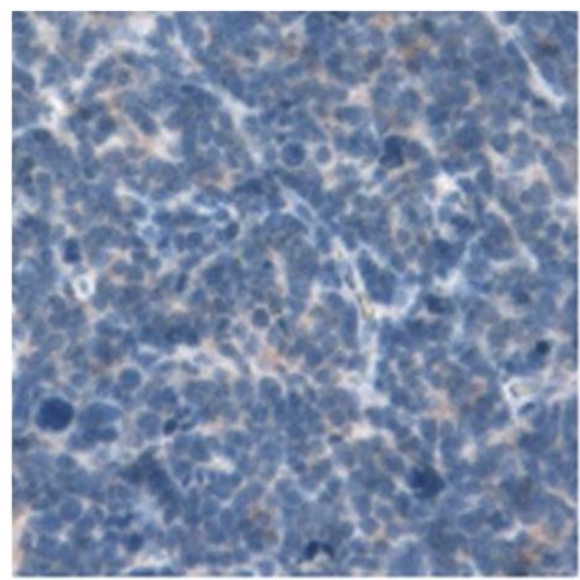

B Necrotic area

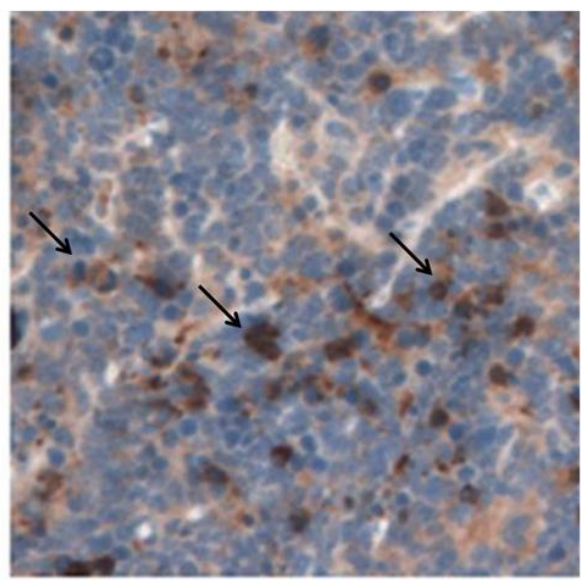

Figure 3.43: Cleaved caspase 3 staining in necrotic tumor tissue. Tumors isolated from TRAMPFVB mice showed next to (A) normal tumor tissue also (B) large necrotic areas. Necrotic tissue showed unspecific staining, so no conclusion about the expression of the tested proteins could be drawn. Representative sections of tumor tissue of $n=5$ sections of untreated and $10 \mathrm{mg} / \mathrm{kg}$ amygdalintreated mice are shown. Pictures were taken under the fluorescence microscope at $100 \mathrm{x}$ magnification.

The unspecific staining was caused by large necrotic areas in the majority of tumor specimens. To investigate if there are differences in necrosis formation between amygdalintreated TRAMP-FVB mice $(10 \mathrm{mg} / \mathrm{kg}$ amygdalin, $100 \mathrm{mg} / \mathrm{kg}$ amygdalin and $100 \mathrm{mg} / \mathrm{kg}$ 
amygdalin purified, from both curative and preventive cohorts) and control-treated TRAMPFVB mice ( $0 \mathrm{mg} / \mathrm{kg}$ amygdalin, from both curative and preventive cohorts), the ratio of the necrotic area compared to the whole tumor area was calculated. Therefore, the cellSens software (Olympus) was used to define and measure non-necrotic and necrotic areas on PCa sections (Fig. 3.44 A) (see 2.23).

Using this analysis $100 \mathrm{mg} / \mathrm{ml}$ amygdalin curative- and preventive-treated mice showed significant increased occurrence of tumor necrosis (Fig. 3.44 B, C). 


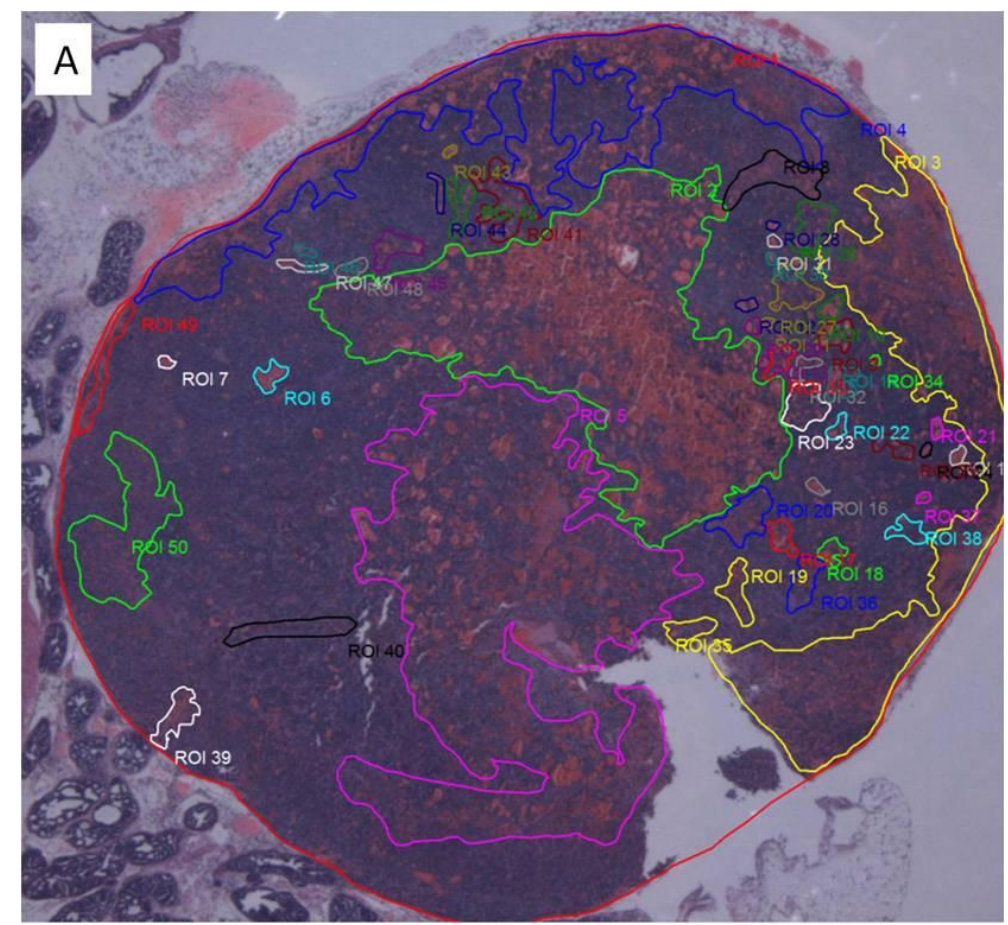

B
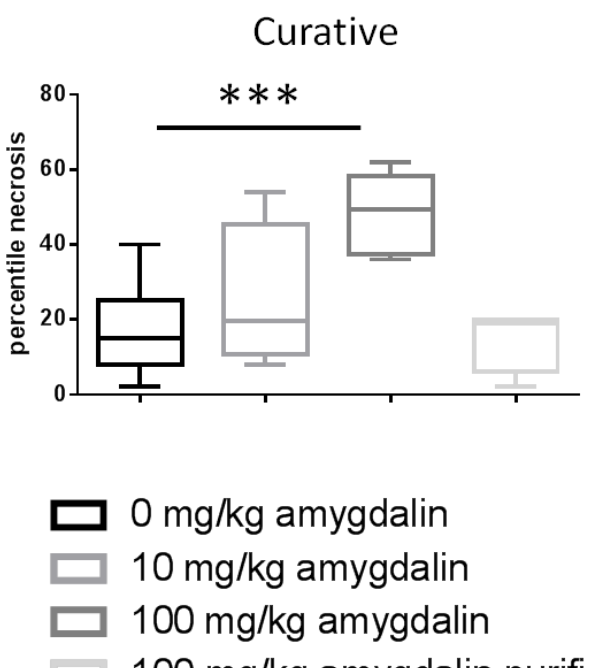

$0 \mathrm{mg} / \mathrm{kg}$ amygdalin $10 \mathrm{mg} / \mathrm{kg}$ amygdalin $100 \mathrm{mg} / \mathrm{kg}$ amygdalin $100 \mathrm{mg} / \mathrm{kg}$ amygdalin purified $\mathrm{n}=4$
C

Preventive

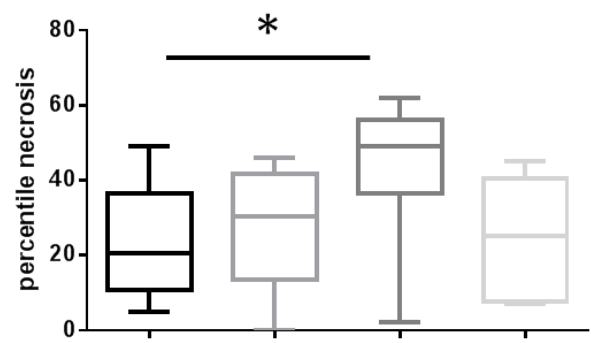

Curative Preventive

$\begin{array}{ll}\mathrm{n}=11 & \mathrm{n}=12 \\ \mathrm{n}=4 & \mathrm{n}=8 \\ \mathrm{n}=6 & \mathrm{n}=8 \\ \mathrm{n}=4 & \mathrm{n}=6\end{array}$

Figure 3.44: Measurement of necrotic areas in TRAMP-FVB PCa specimens. (A) Depicted is a representative hemalum and eosin stained tumor sample. Photographs of $\mathrm{PCa}$ specimens of amygdalin-treated and control mice were taken at 10x magnification. Necrotic and total tumor area was defined (see color-marked areas) calculated using the cellSens software (Olympus). Necrotic area was set relative to total tumor area. Depicted are the mean values of $0 \mathrm{mg} / \mathrm{kg}, 10 \mathrm{mg} / \mathrm{kg}$, $100 \mathrm{mg} / \mathrm{kg}$ and $100 \mathrm{mg} / \mathrm{kg}$ purified amygdalin-treated animals each for (B) curative or (C) preventive treatment in a box plot. $100 \mathrm{mg} / \mathrm{ml}$ amygdalin curative- and preventive-treated mice showed significant increased occurrence of necrosis. Significance was calculated using the student's t-test. $p<0.05$, ** $p<0.01,{ }^{* * *} p<0.0001$ compared to untreated control. 


\subsubsection{In vitro analyses of the effect of amygdalin on murine $\mathrm{PCa}$ cells}

The in vivo experiments did not reveal definite and precise information about the cellular and molecular mechanisms how amygdalin treatment could influence PCa cell growth, survival and apoptosis. Furthermore, the massive induction of tumor necrosis after amygdalin treatment could falsify subsequent IHC and western blot analysis. Therefore, two murine primary PCa cell lines were used to elucidate the effect of amygdalin treatment on PCa cells (see 3.1.1.4).

\subsubsection{Effect of amygdalin on cell survival, cytotoxicity and apoptosis induction on $\mathrm{T} 244$ and $2 \mathrm{E}$ cells}

Cell survival, cytotoxic effects and apoptosis induction upon amygdalin treatment was analyzed. Amygdalin concentration were used as described in Makarevic et al. (2016). Additionally, $20 \mathrm{mg} / \mathrm{ml}$ amygdalin were used, which was the highest applicable concentration limited by solubility. T244 and $2 \mathrm{E}$ cells were treated with $0 \mathrm{mg} / \mathrm{ml}, 1 \mathrm{mg} / \mathrm{ml}, 5 \mathrm{mg} / \mathrm{ml}$, $10 \mathrm{mg} / \mathrm{ml}$ and $20 \mathrm{mg} / \mathrm{ml}$ amygdalin and cell survival, cytotoxicity and apoptosis induction were measured after $24 \mathrm{~h}, 48 \mathrm{~h}, 72 \mathrm{~h}$ and $6 \mathrm{~d}$.

Amygdalin reduced cell survival in a time- and dose-dependent manner in both cell lines. In T244 cells cell survival was reduced by $51 \%$ after $6 \mathrm{~d}$ treatment with $20 \mathrm{mg} / \mathrm{ml}$ amygdalin. $2 \mathrm{E}$ cells showed a reduction by $56 \%$ after $6 \mathrm{~d}$ treatment with $20 \mathrm{mg} / \mathrm{ml}$ amygdalin (Fig. 3.45 A, B). Both cell lines did not show cytotoxic effects upon amygdalin treatment, instead cytotoxicity reduced in both cell lines after amygdalin treatment (T244 by $31 \%$, 2E by $45 \%$ after $6 \mathrm{~d}$ treatment with $20 \mathrm{mg} / \mathrm{ml}$ amygdalin) (Fig. $3.45 \mathrm{C}$, D). Amygdalin did not induce apoptosis in T244 or 2E cells, i.e. apoptosis measurement showed rather declined apoptosis compared to the untreated control (T244 by $60 \%$, 2E by $83 \%$ after $6 \mathrm{~d}$ treatment with $20 \mathrm{mg} / \mathrm{ml}$ amygdalin) (Fig. 3.45 E, F). 
A

T244

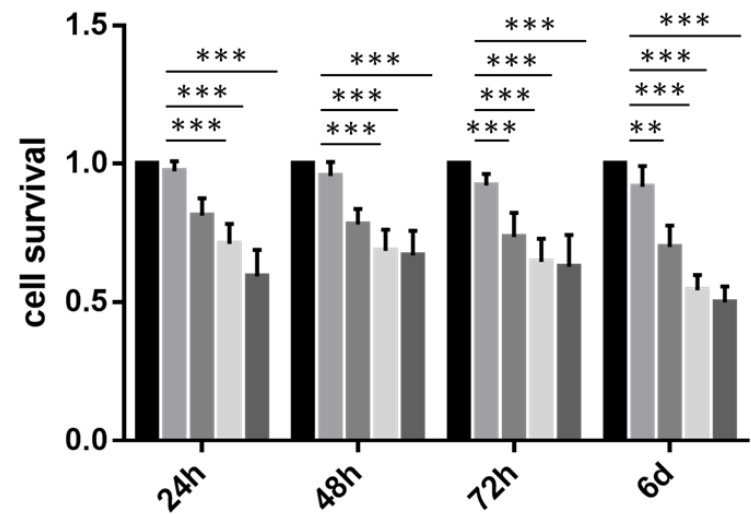

C

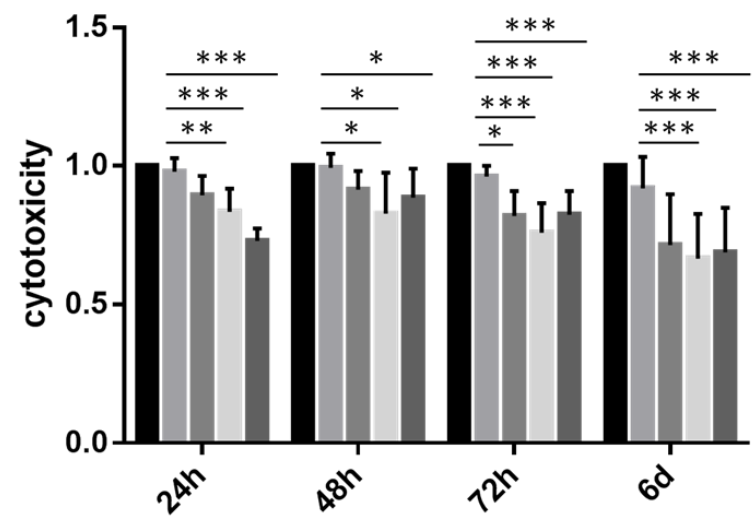

E

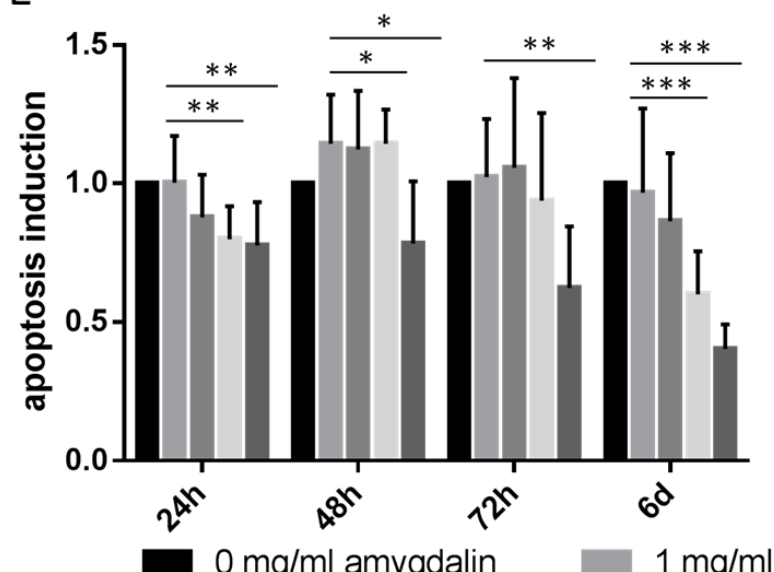

B 2E
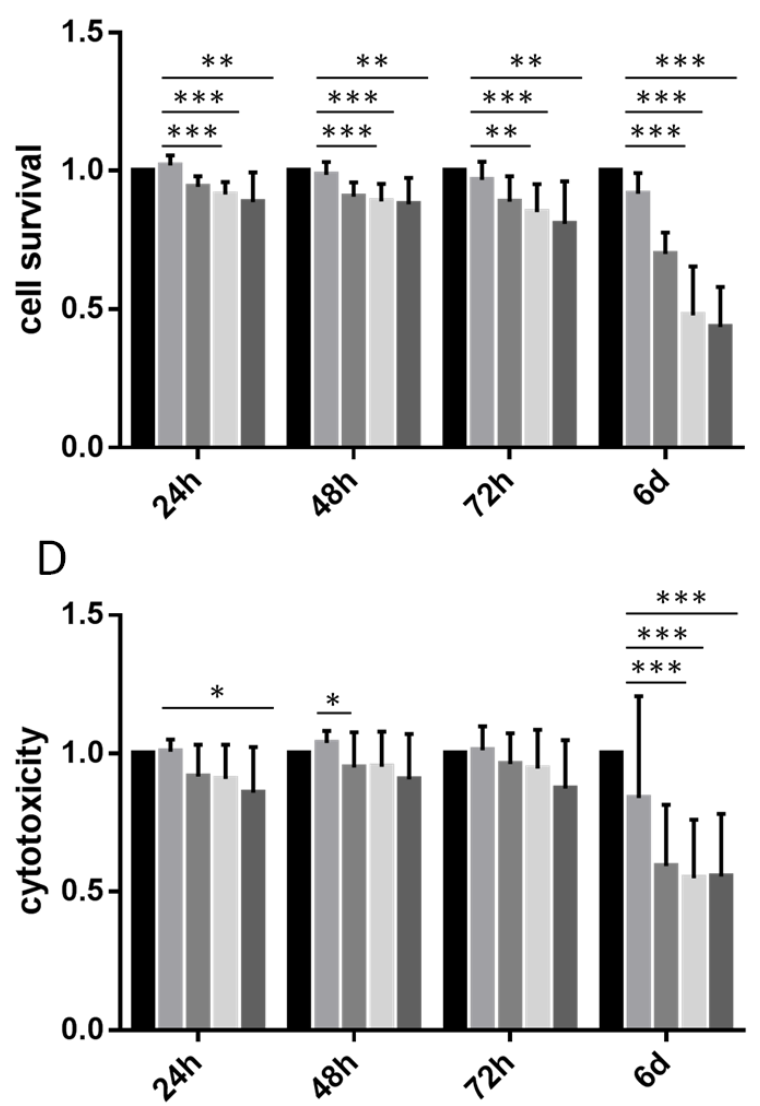

F

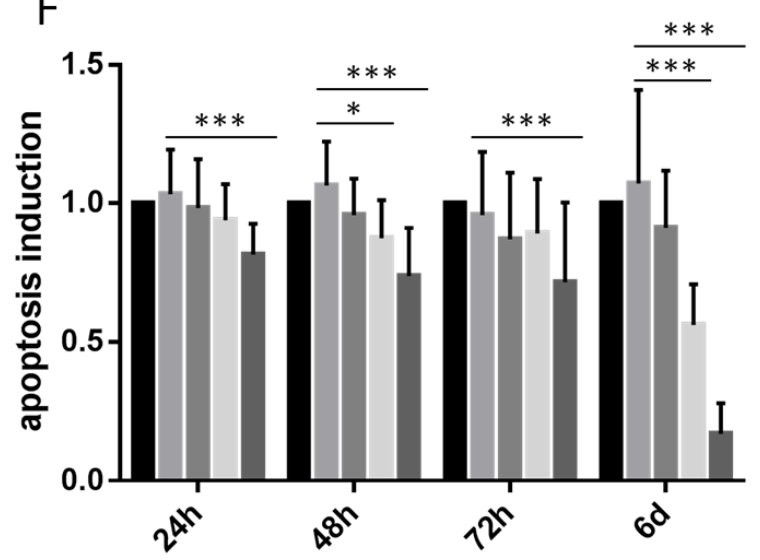

$5 \mathrm{mg} / \mathrm{ml}$ amygdalin

Figure 3.45: Cell survival, cytotoxicity and induction of apoptosis after amygdalin treatment in murine primary PCa cell lines T244 and 2E. T244 and 2E cells showed a time- and dose-dependent reduction of (A and $\mathbf{B})$ cell survival, but ( $\mathbf{C}$ and $\mathbf{D})$ no cytotoxic effects or ( $\mathbf{E}$ and $\mathbf{F}$ ) apoptosis upon amygdalin treatment. The data represent the mean \pm s.d. of three independent experiments, which were performed in duplicate. Significance was calculated using the student's t-test. ${ }^{*} p<0.05$, ** $p<0.01,{ }^{* \star *} p<0.0001$ compared to control cells. 


\subsubsection{Analysis of protein integrity of amygdalin-treated primary PCa cell lines T244 and 2E}

The quality of proteins isolated from tumor tissues of amygdalin-treated mice declined with increasing amygdalin concentrations. To test if this phenomenon also occurs in cell culture, murine PCa cell lines T244 and 2E were treated with $0 \mathrm{mg} / \mathrm{ml}, 5 \mathrm{mg} / \mathrm{ml}$ and $20 \mathrm{mg} / \mathrm{ml}$ amygdalin, respectively and protein was isolated after $72 \mathrm{~h}$. Protein concentration was measured and $15 \mu \mathrm{g}$ protein was immunoblotted and incubated with a with HSC70-specific antibody.

Again, protein quality declined when cells were incubated with the highest concentration (20 mg/ml) of amygdalin (Fig. 3.46). Therefore, western blot analysis was not an appropriate analysis technique for amygdalin-induced effects.

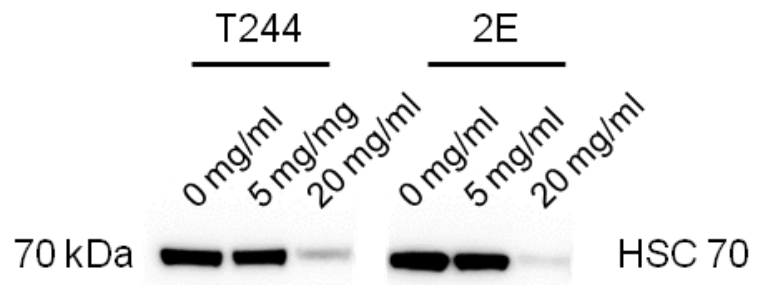

Figure 3.46: Protein integrity of amygdalin-treated primary PCa cell lines T244 and 2E. Murine PCa cell lines T244 and 2E were treated with $0 \mathrm{mg} / \mathrm{ml}, 5 \mathrm{mg} / \mathrm{ml}$ and $20 \mathrm{mg} / \mathrm{ml}$ amygdalin, respectively and protein was isolated after $72 \mathrm{~h}$. Protein concentration was measured using the Bradford assay and $15 \mu \mathrm{g}$ protein were loaded for immunoblotting. Signal was detected using a HSC70-specific antibody. Treatment with amygdalin at a concentration of $20 \mathrm{mg} / \mathrm{ml}$ induced protein degradation, therefore valid results could not be obtained with these protein samples. The data represent two independent experiments, which were performed in duplicate. A representative western blot is shown.

\subsubsection{Effect of amygdalin on the invasive potential of primary PCa cell lines T244 and 2E}

The invasive potential of cells is a hallmark of aggressive cancer growth (Hanahan, Weinberg 2011). To analyze if amygdalin has an effect on cancer cell invasiveness T244 and $2 \mathrm{E}$ cells were pretreated with $20 \mathrm{mg} / \mathrm{ml}$ amygdalin for $24 \mathrm{~h}$ and allowed to grow on matrigelcoated invasion chambers for another $24 \mathrm{~h}$. Invaded cells were fixed, stained and cell number was counted in five randomly visual fields under the microscope (see 2.20.3). Amygdalin treatment significantly reduced the number of invaded cells by $75 \%$ in T244 cells. In contrast, invasiveness of 2E cells was not changed after amygdalin treatment (Fig 3.47). 
A

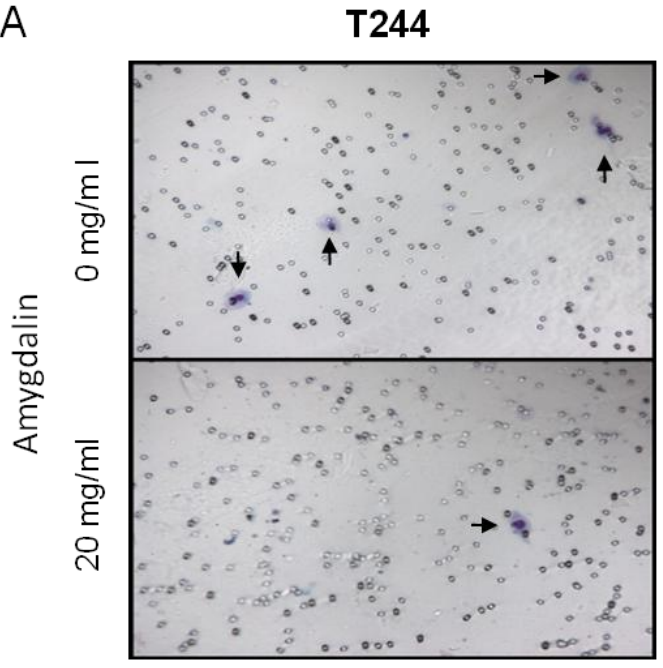

C

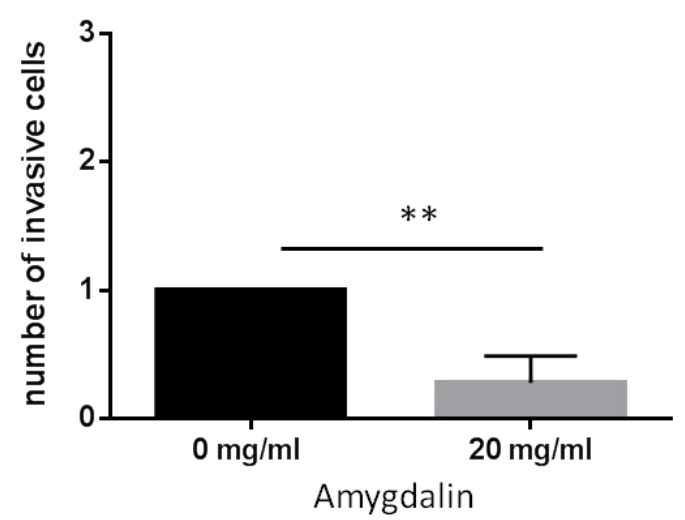

B

$2 \mathrm{E}$

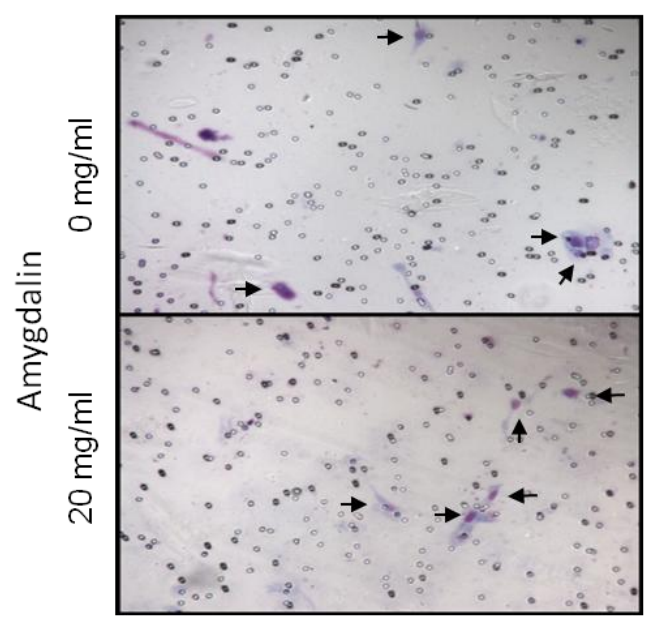

2E

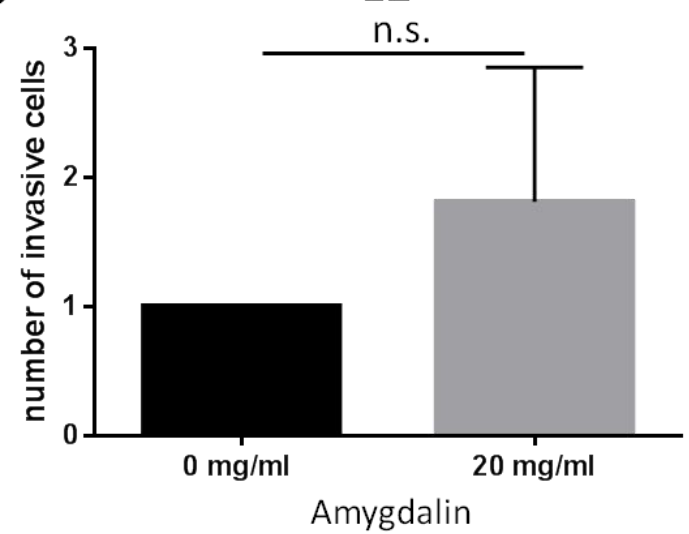

Figure 3.47: Invasiveness of primary PCa cell lines T244 and 2E after amygdalin treatment. (A) T244 and (B) 2E cells were treated with $0 \mathrm{mg} / \mathrm{ml}$ or $20 \mathrm{mg} / \mathrm{ml}$ amygdalin and incubated for $24 \mathrm{~h}$ on an invasion chamber. Invasive cells (indicated by arrows in $\mathbf{A}$ and $\mathbf{B}$ ) were counted in five randomly taken pictures under the fluorescent microscope at 40x magnification. (C) T244 cells showed significant reduced number of invasive cells after amygdalin treatment. (D) Invasiveness of $2 \mathrm{E}$ cells was unchanged after amygdalin treatment. The data represent the mean \pm s.d. of three independent experiments. Significance was calculated using the student's t-test. ${ }^{*} p<0.05,{ }^{* *} p<0.01,{ }^{* * *} p<0.0001$ compared with amygdalin control.

\subsubsection{Morphological analysis of amygdalin-treated T244 and 2E cells}

Amygdalin treatment induced cell survival reduction, but no induction of apoptosis or cell death in T244 and 2E cells. To further investigate the underlying mechanism for the cell survival reduction, morphological changes of murine PCa cells upon amygdalin treatment were analyzed. T244 and 2E cells were treated with $0 \mathrm{mg} / \mathrm{ml}, 5 \mathrm{mg} / \mathrm{ml}$ and $20 \mathrm{mg} / \mathrm{ml}$ amygdalin and cell morphology was daily documented for $10 \mathrm{~d}$.

Both T244 and 2E cells showed morphological changes due to amygdalin treatment. These changes became apparent with increasing amygdalin concentration and experimental time course. After $10 \mathrm{~d}$ of amygdalin treatment the cell shape was affected, i.e. the usually round 
or elongated PCa cells showed spine formation (see arrows) or a flatted, undefined cellular shape (Fig. 3.48, see arrowheads). Furthermore, it could be observed that with ongoing amygdalin treatment cell number declined dependent on amygdalin concentration.

A

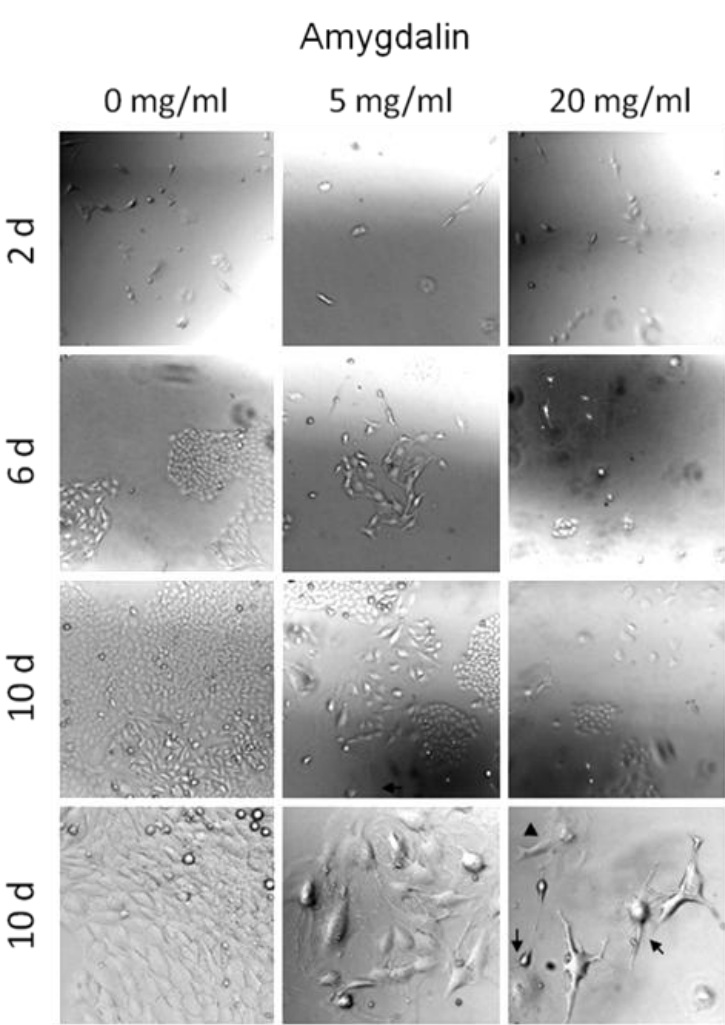

$\mathrm{B}$ $2 \mathrm{E}$

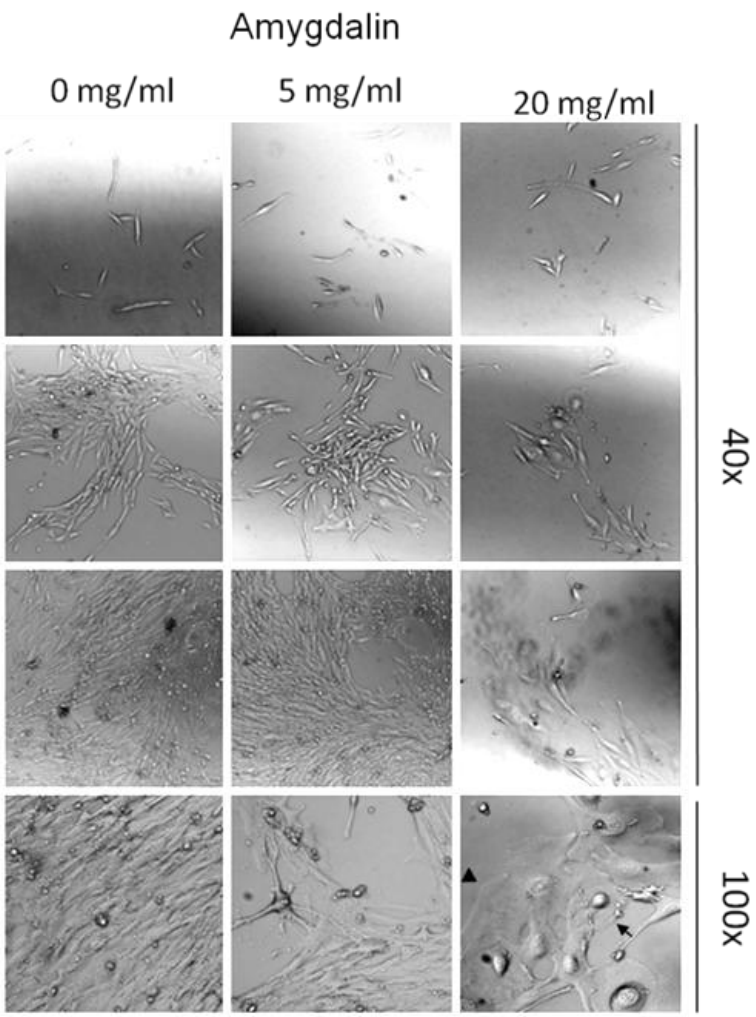

Figure 3.48: Morphological changes in primary PCa cell lines T244 and 2E upon amygdalin treatment. (A) T244 and (B) 2E cells were treated with $0 \mathrm{mg} / \mathrm{ml}, 5 \mathrm{mg} / \mathrm{ml}$ and $20 \mathrm{mg} / \mathrm{ml}$ amygdalin for $10 \mathrm{~d}$ and analyzed daily under the inverse microscope. Cell morphology changes were observed in a time- and dose-dependent manner. Amygdalin-treated cells formed long spines or lost cell shape. Representative pictures of three independent experiments are shown.

\subsubsection{Analysis of cell number in amygdalin-treated primary PCa cell lines T244 and 2E}

To validate the result of cell survival reduction in amygdalin-treated primary PCa cell lines T244 and 2E, cells were treated with $0 \mathrm{mg} / \mathrm{ml}, 5 \mathrm{mg} / \mathrm{ml}$ and $20 \mathrm{mg} / \mathrm{ml}$ amygdalin for $3 \mathrm{~d}, 5 \mathrm{~d}$ or $7 \mathrm{~d}$ and nuclei were stained. Next, number of cells was counted in five random visual fields under the microscope and compared to the numbers of untreated control cells.

The treatment with $20 \mathrm{mg} / \mathrm{ml}$ amygdalin resulted in reduced cell counts up to $67 \%$ in T244 cells and $70 \%$ in 2 E cells (Fig. 3.49). 
A
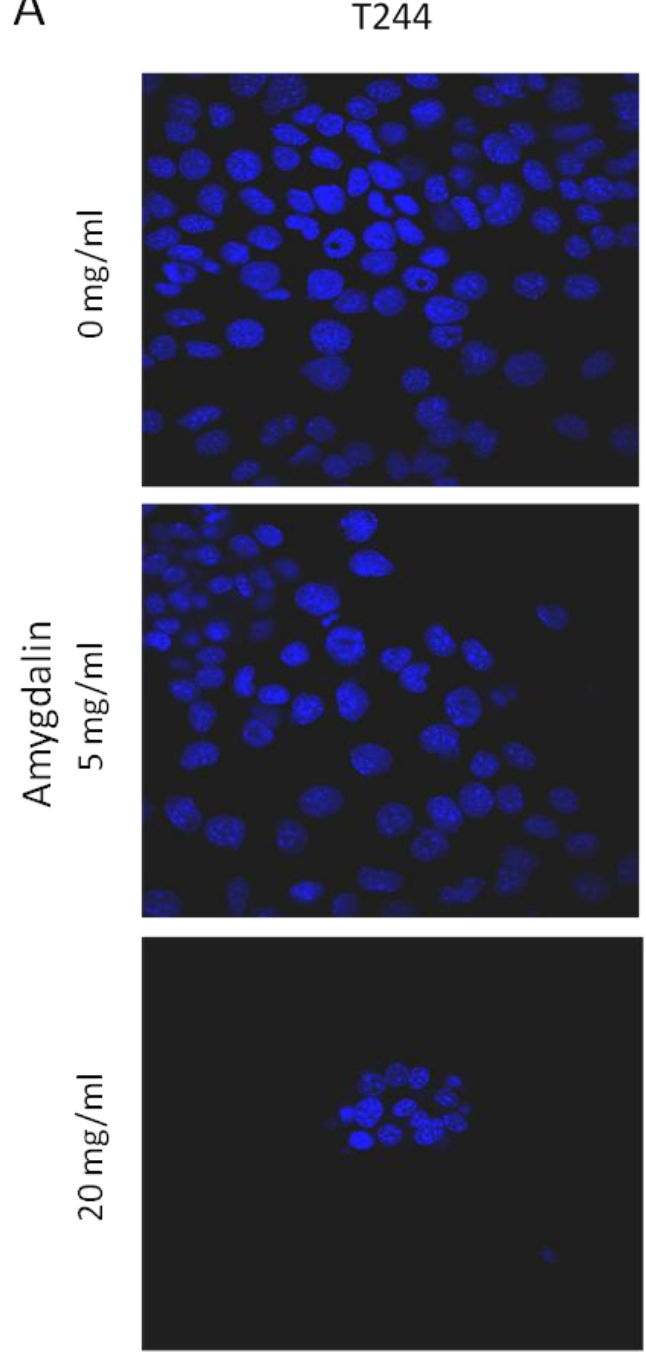

T244

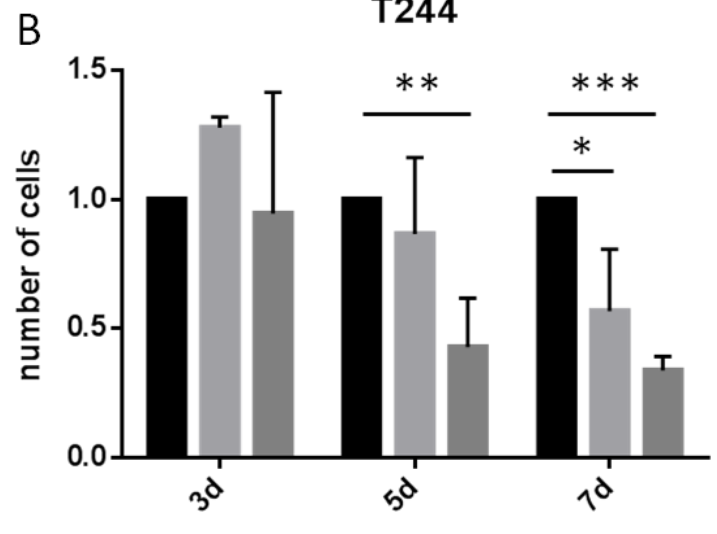

$0 \mathrm{mg} / \mathrm{ml}$ amydalin

T244
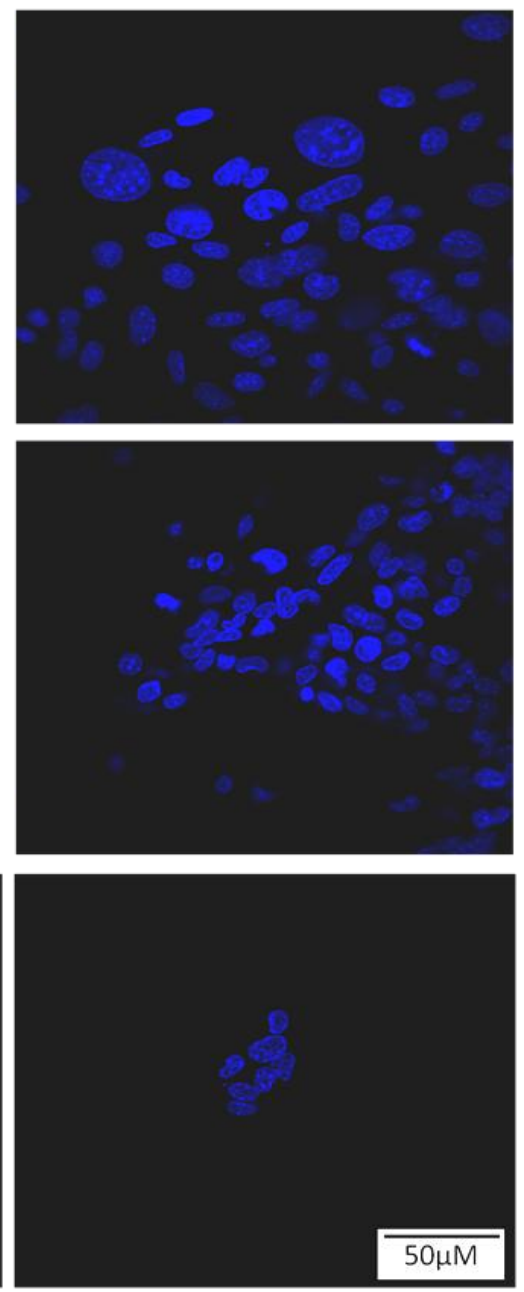

C

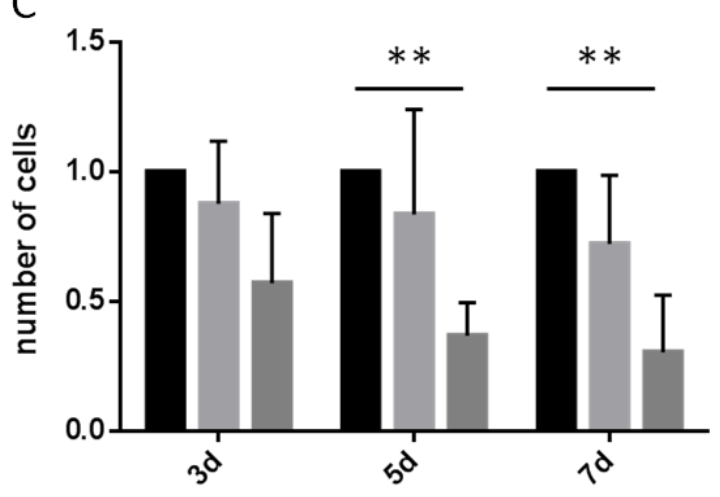

$5 \mathrm{mg} / \mathrm{ml}$ amygdalin
$2 \mathrm{E}$

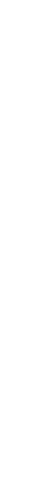


experiments. Significance was calculated using the student's t-test. ${ }^{*} p<0.05,{ }^{* *} p<0.01,{ }^{* * *} p<0.0001$ compared with control cells.

\subsubsection{Analysis of proliferation in amygdalin-treated primary PCa cell lines T244 and 2E}

To investigate if the decreased cell number is associated with decreased proliferation, T244 and $2 \mathrm{E}$ cells were treated with $5 \mathrm{mg} / \mathrm{ml}$ and $20 \mathrm{mg} / \mathrm{ml}$ amygdalin and the proliferation marker KI67 was stained. Expression was analyzed in five randomly taken pictures using the fluorescent microscope. Stained and unstained cells were counted and the relative number of stained cells was calculated.

Expression of KI67 did not alter due to amygdalin treatment in T244 cells, whereas $2 \mathrm{E}$ cells showed $70 \%$ less KI67 expression after $7 \mathrm{~d}$ of $20 \mathrm{mg} / \mathrm{ml}$ amygdalin treatment (Fig. 3.50).

A
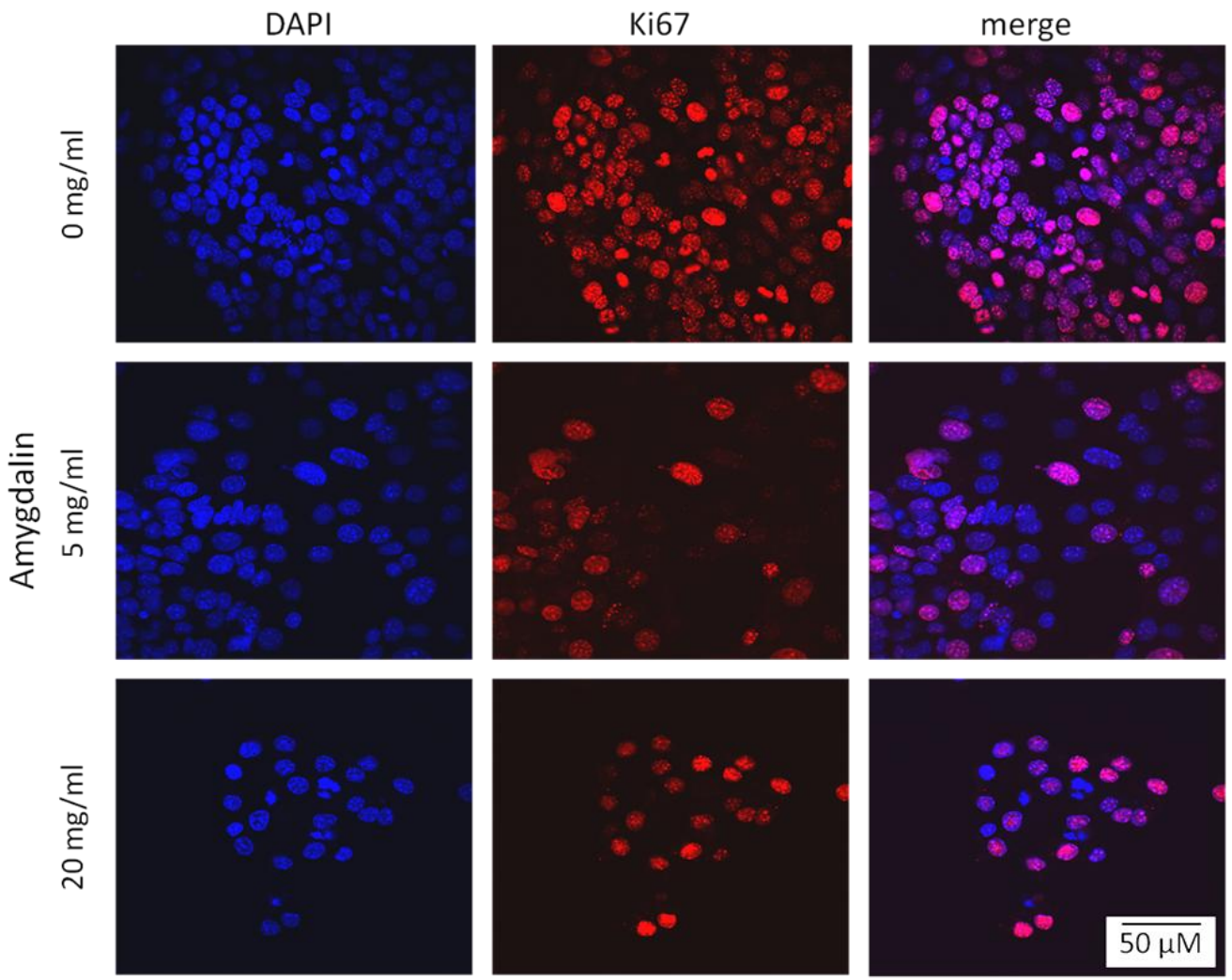
B

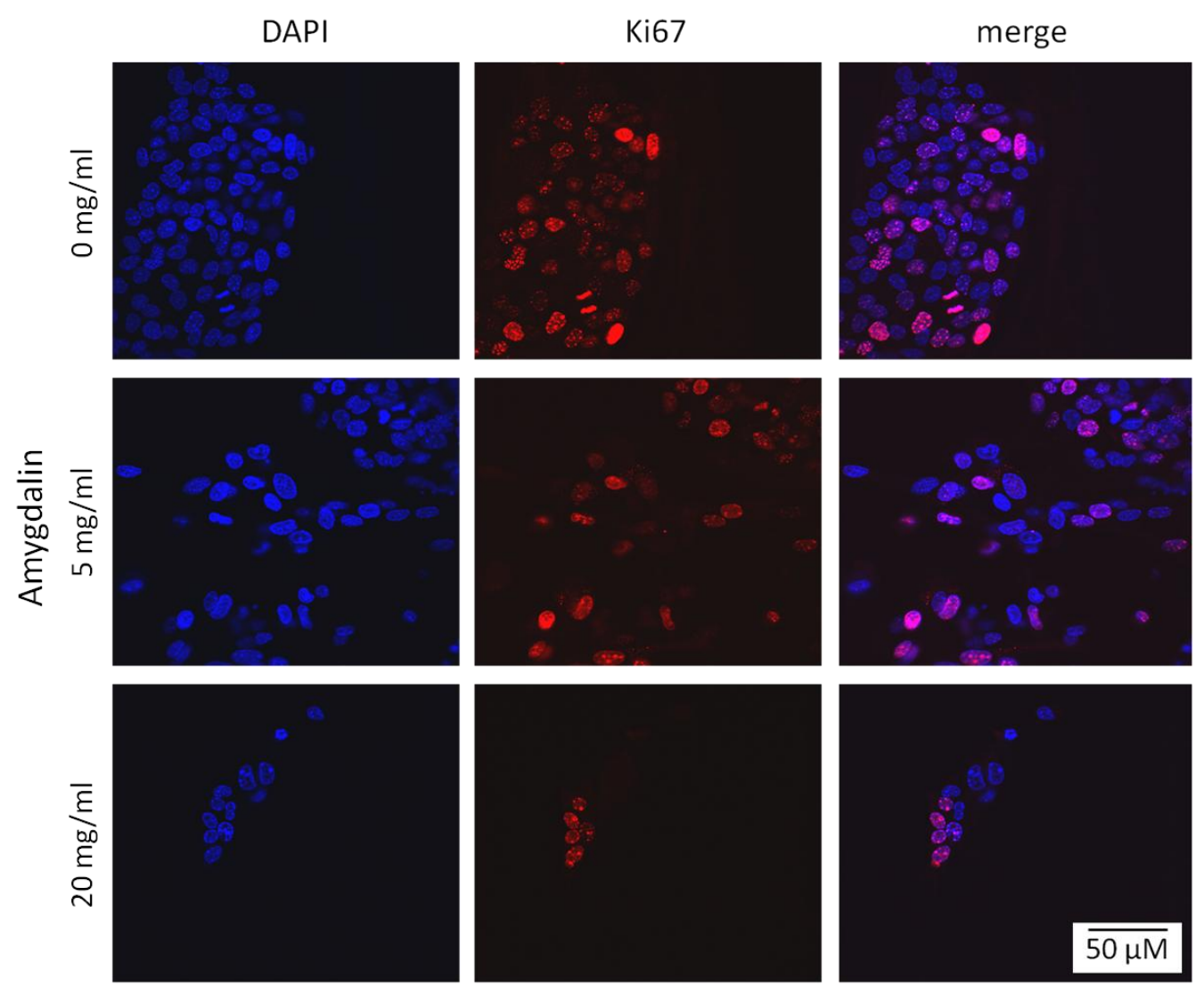

C

T244

D

2E
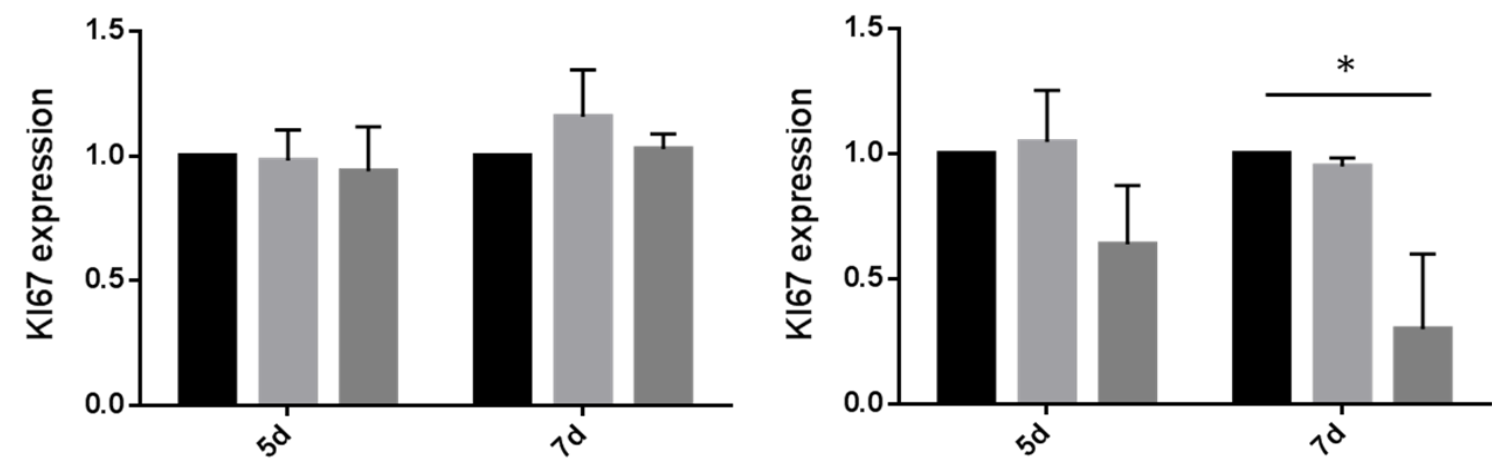

$0 \mathrm{mg} / \mathrm{ml}$ amydalin

$5 \mathrm{mg} / \mathrm{ml}$ amygdalin

$20 \mathrm{mg} / \mathrm{ml}$ amygdalin

Figure 3.50: Expression of proliferation marker KI67 in amygdalin-treated primary PCa cell lines T244 and 2E. (A) T244 and (B) 2E cells were treated with $0 \mathrm{mg} / \mathrm{ml}, 5 \mathrm{mg} / \mathrm{ml}$ and $20 \mathrm{mg} / \mathrm{ml}$ amygdalin for $5 \mathrm{~d}$ and $7 \mathrm{~d}$ and the proliferation marker KI67 was detected using immunofluorescence (stained in red). Expression was analyzed in five randomly taken pictures under the fluorescent microscope. (A and C) T244 cells showed no altered expression of KI67, but (B and D) $2 E$ cells showed $70 \%$ less $\mathrm{KI} 67$ expression after $7 \mathrm{~d}$ of $20 \mathrm{mg} / \mathrm{ml}$ amygdalin treatment. The data represent the mean \pm s.d. of three independent experiments. Significance was calculated using the student's t-test. ${ }^{*} p<0.05$, ** $p<0.01,{ }^{* * *} p<0.0001$ compared with control. 


\subsubsection{Analysis of senescence induction in amygdalin-treated primary $\mathrm{PCa}$ cell lines T244 and 2E}

The reduction of viable murine PCa cells without induction of cell death together with the cell morphological changes indicates that these cells might enter a senescent cell stage. To visualize senescent cells the senescent-associated $\beta$-galactosidase (SA $\beta$-gal) expression was detected. Primary PCa cell lines T244 and $2 \mathrm{E}$ were treated with $0 \mathrm{mg} / \mathrm{ml}, 5 \mathrm{mg} / \mathrm{ml}$ and $20 \mathrm{mg} / \mathrm{ml}$ amygdalin for $5 \mathrm{~d}$ and $7 \mathrm{~d}$, respectively and activity of $\beta$-galactosidase was visualized (see 2.20.4). Subsequently, stained and unstained cells were counted and the relative number of stained cells was calculated.

Both cell lines showed increased expression of SA $\beta$-gal after $7 \mathrm{~d}$ of $20 \mathrm{mg} / \mathrm{ml}$ amygdalin treatment. SA $\beta$-gal expression increased 2 -fold in T244 cells and 5 -fold in $2 \mathrm{E}$ cells after $7 \mathrm{~d}$ (Fig. 3.51). 
A

T244

$5 d$

$7 d$

d
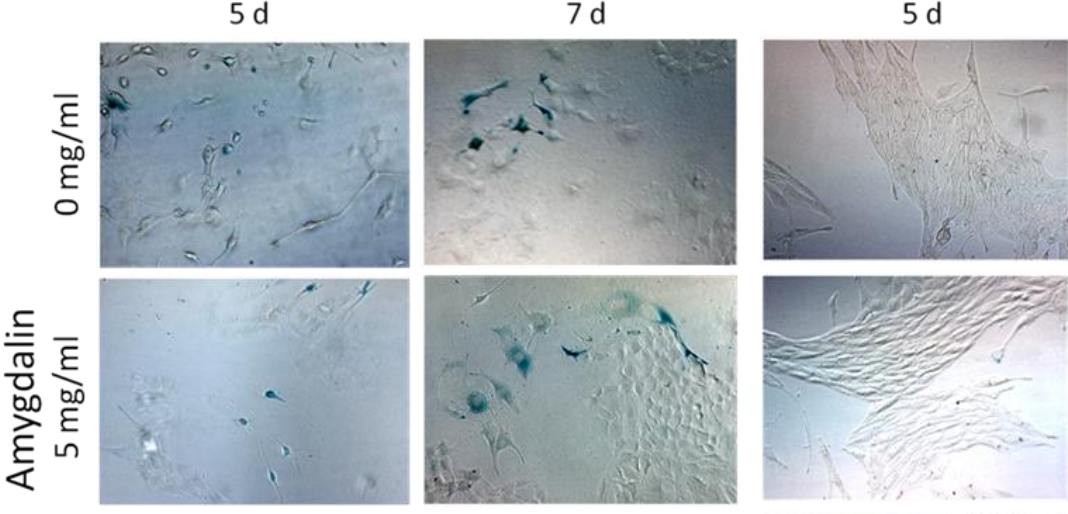

$2 \mathrm{E}$
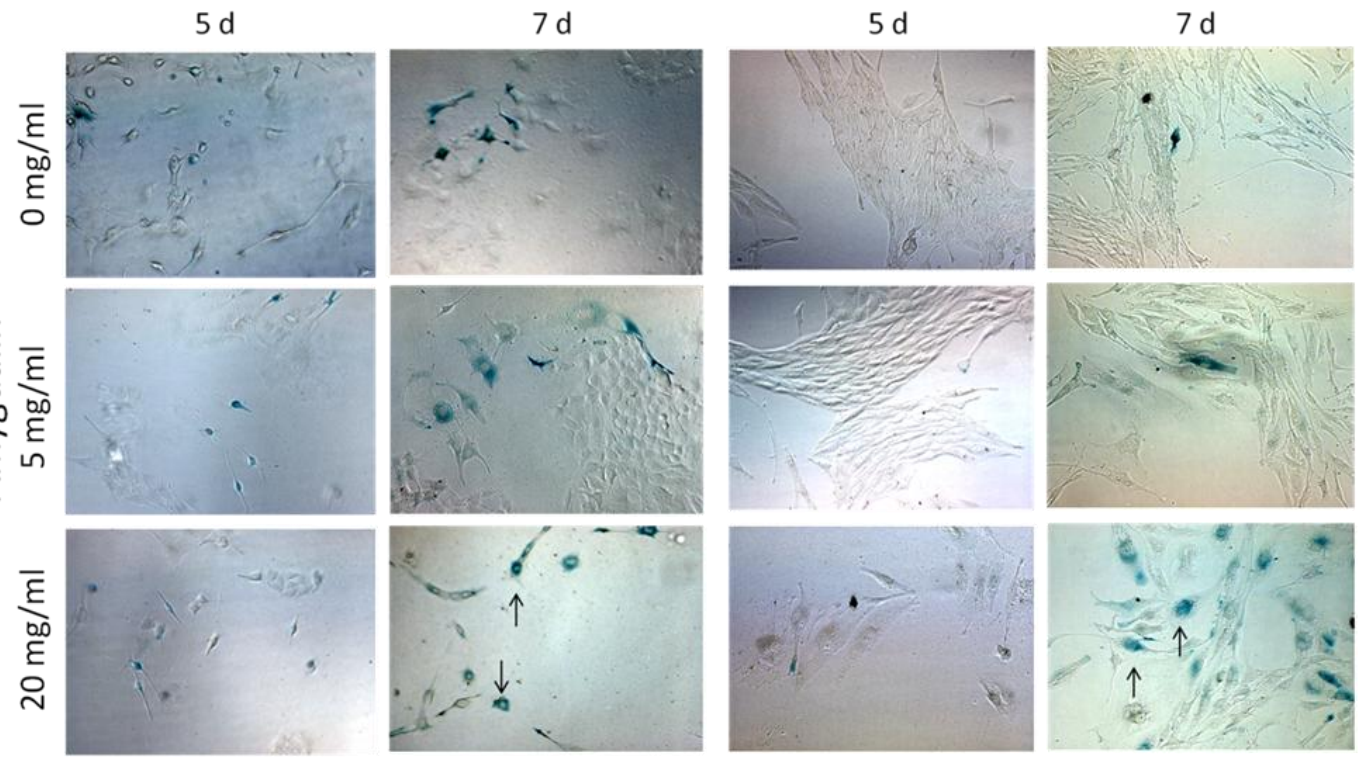

B

T244

C
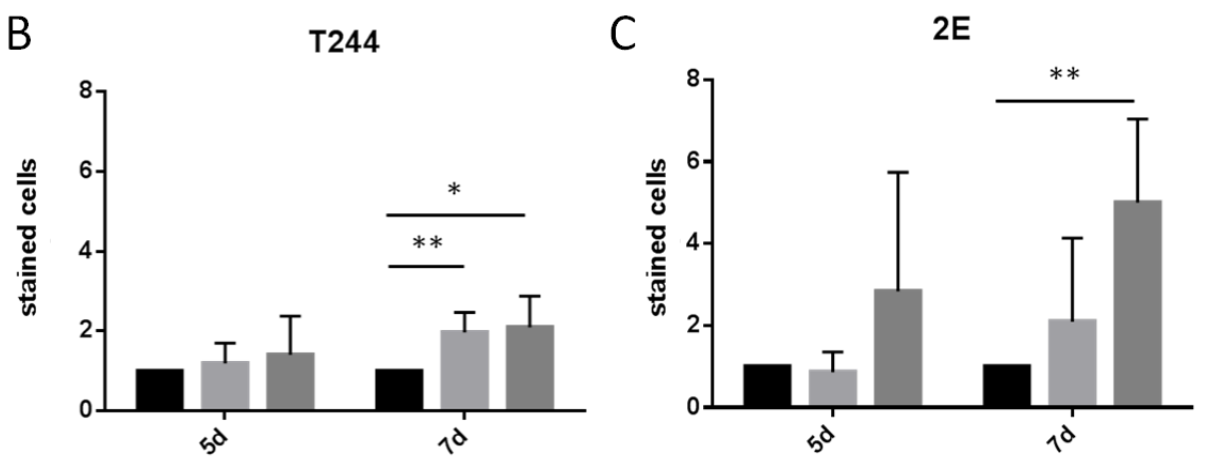

$0 \mathrm{mg} / \mathrm{ml}$ amydalin

$5 \mathrm{mg} / \mathrm{ml}$ amygdalin

$20 \mathrm{mg} / \mathrm{ml}$ amygdalin

Figure 3.51: SA $\beta$-gal staining in amygdalin-treated primary PCa cell lines T244 and 2E. (A) T244 and 2E cells were treated with $0 \mathrm{mg} / \mathrm{ml}, 1 \mathrm{mg} / \mathrm{ml}, 5 \mathrm{mg} / \mathrm{ml}, 10 \mathrm{mg} / \mathrm{ml}$ or $20 \mathrm{mg} / \mathrm{ml}$ amygdalin and $\beta$-galactosidase was stained (blue) after $5 \mathrm{~d}$ and $7 \mathrm{~d}$. Pictures were taken under the inverse microscope at 40x magnification. Both, (B) T244 and (C) 2E cells showed increased staining after $7 \mathrm{~d}$ of amygdalin treatment (2-fold in T244 and 5-fold in 2E cells). The data represent the mean \pm s.d. of three independent experiments. Significance was calculated using the student's t-test. * $p<0.05$, ** $p<0.01,{ }^{* * *} p<0.0001$ compared with control.

\subsubsection{Analysis of senescence inducing factors in amygdalin-treated primary PCa cell lines T244 and 2E}

Senescence can be induced for example by DNA double strand breaks (DSB) or mitotic catastrophe (Levine, Oren 2009; Yen, Klionsky 2008). To identify the underlying cause for senescence induction in amygdalin-treated T244 and 2E PCa cells markers for DSB (H2AX) and mitotic catastrophe (damaged nuclei) were analyzed. 


\subsection{Detection of yH2Ax expression in amygdalin-treated primary PCa cell lines T244 and $2 E$}

As a marker for DSB the protein $\mathrm{yH} 2 \mathrm{Ax}$ was stained by immunofluorescence. T244 and 2E PCa cells were treated with $0 \mathrm{mg} / \mathrm{ml}, 5 \mathrm{mg} / \mathrm{ml}$ or $20 \mathrm{mg} / \mathrm{ml}$ amygdalin for $3 \mathrm{~d}$ or $7 \mathrm{~d}$. $\mathrm{yH} 2 \mathrm{Ax}$ stained cells were counted in five randomly taken pictures taken under the fluorescent microscope. Stained and unstained cells were counted and the relative number of stained cells was calculated.

After $7 \mathrm{~d}$ treatment with $20 \mathrm{mg} / \mathrm{ml}$ amygdalin the number of $\mathrm{yH} 2 \mathrm{Ax}$ foci increased 1.5 -fold in T244 and 2E cells (Fig. 3.52).

A

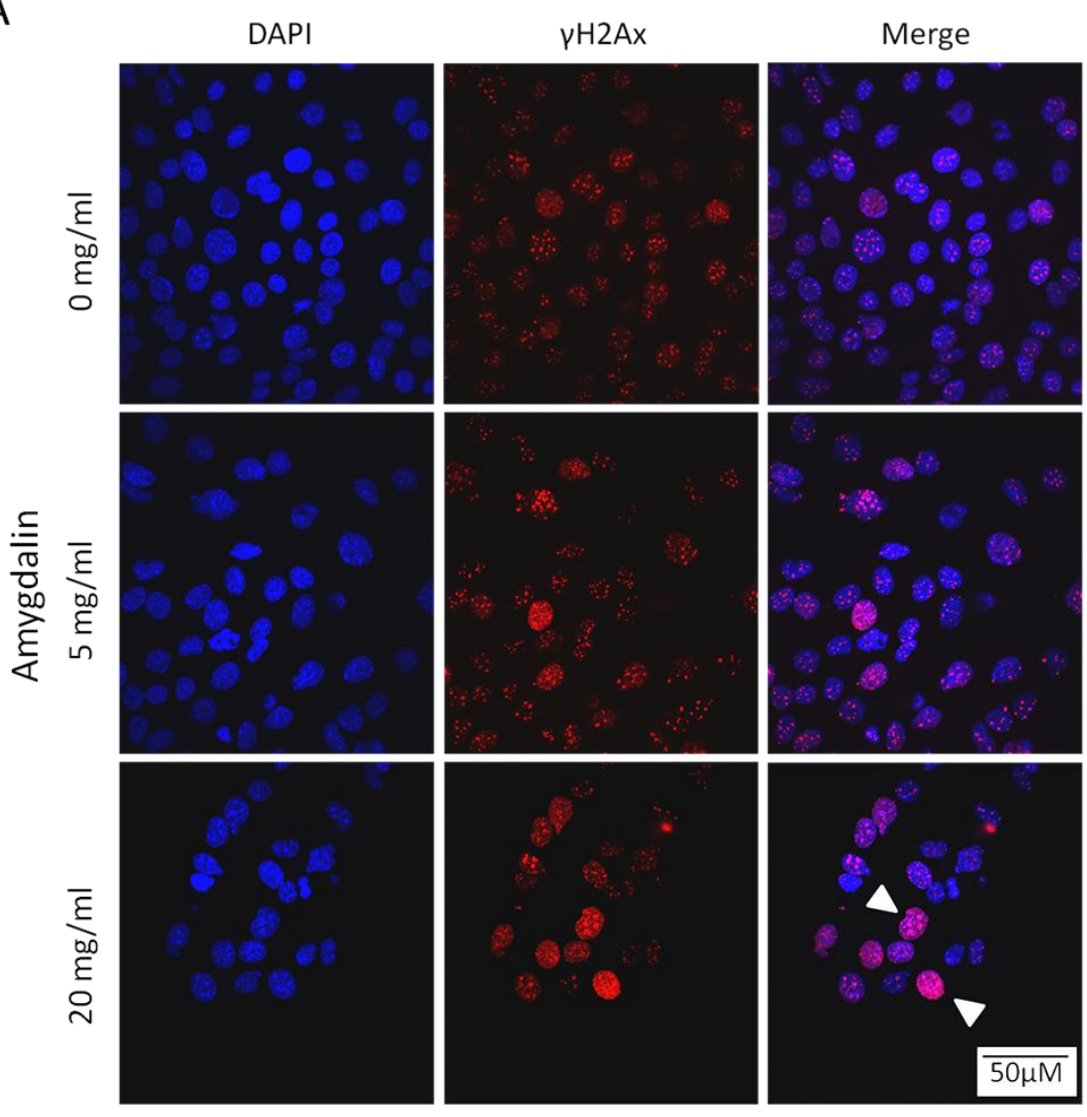




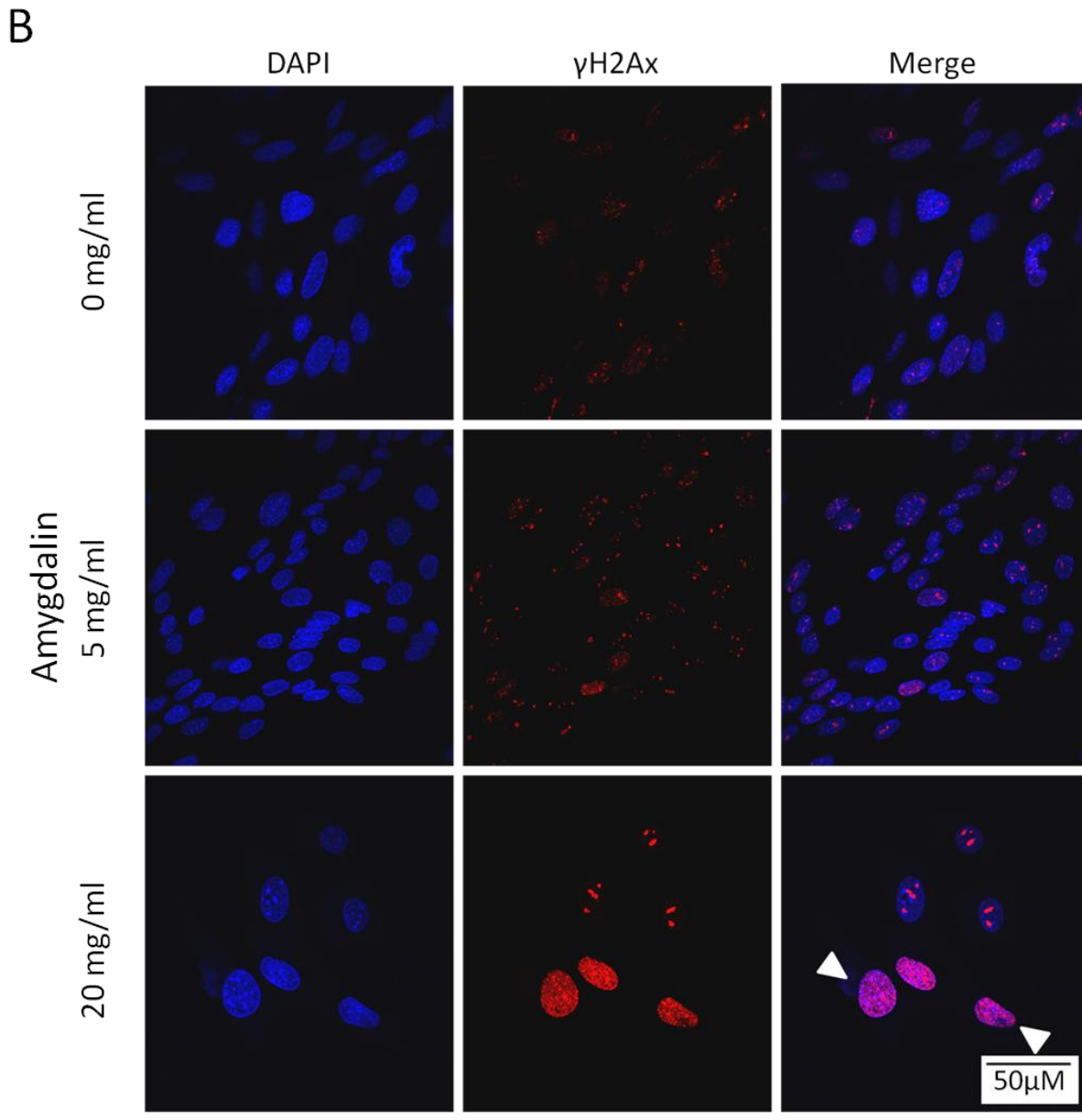

C

T244

D

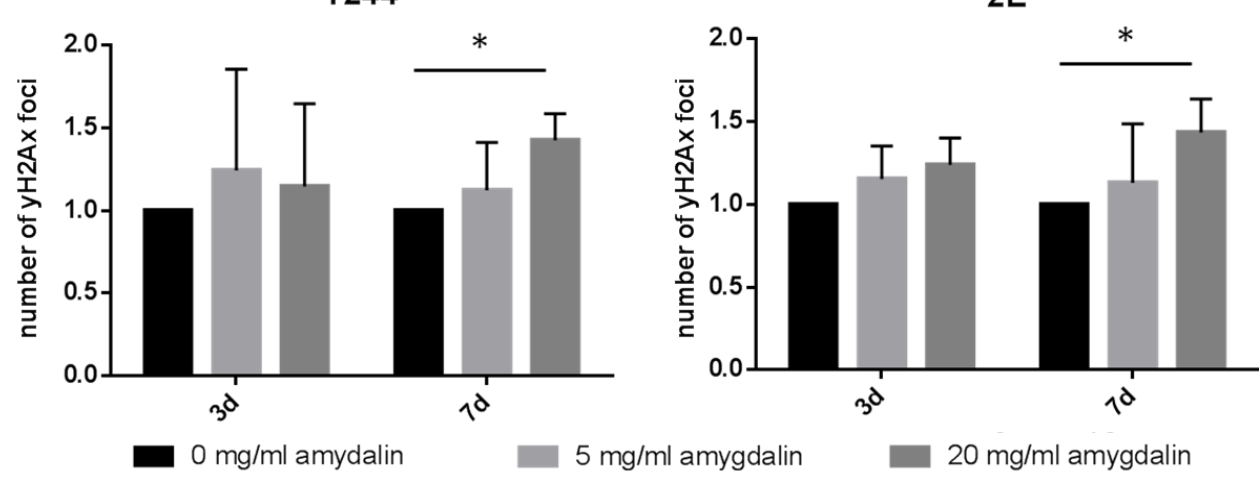

Figure 3.52: DNA double strand breaks (DSB) in primary PCa cell lines T244 and 2E after amygdalin treatment. (A) T244 and (B) $2 E$ cells were treated with $0 \mathrm{mg} / \mathrm{ml}, 5 \mathrm{mg} / \mathrm{ml}$ or $20 \mathrm{mg} / \mathrm{ml}$ amygdalin for $3 \mathrm{~d}$ and $7 \mathrm{~d}$ and $\mathrm{yH} 2 \mathrm{Ax}$ foci were stained (red) and counted in five randomly taken pictures taken under the fluorescent microscope. After $7 \mathrm{~d}$ treatment with $20 \mathrm{mg} / \mathrm{ml}$ amygdalin both (C) T244 and (D) 2E cells showed 1.5-fold increased number of $\mathrm{yH} 2 \mathrm{Ax}$ foci. The data represent the mean \pm s.d. of three independent experiments. Significance was calculated using the student's t-test. ${ }^{*} p<0.05,{ }^{* *} p<0.01,{ }^{* *} p<0.0001$ compared with control. 


\subsection{Detection of mitotic catastrophe in amygdalin-treated primary PCa cell}

\section{lines T244 and 2E}

Mitotic catastrophe is induced by missegregation of chromosomes during mitosis and misshaped cellular nuclei are used as a marker for this phenomenon. Primary PCa cell lines T244 and 2E were treated with $0 \mathrm{mg} / \mathrm{ml}, 5 \mathrm{mg} / \mathrm{ml}$ or $20 \mathrm{mg} / \mathrm{ml}$ amygdalin for $3 \mathrm{~d}, 5 \mathrm{~d}$ or $7 \mathrm{~d}$. Cells were stained with DAPI and misshaped nuclei were counted in five randomly taken pictures using the fluorescent microscope. Damaged and intact nuclei were counted and the relative number of damaged cells was calculated.

Mitotic catastrophe was reduced by $53 \%$ in T244 cells, but increased 2-fold in 2E cells upon treatment with $20 \mathrm{mg} / \mathrm{ml}$ amygdalin for $7 \mathrm{~d}$ (Fig. 3.53). 
A
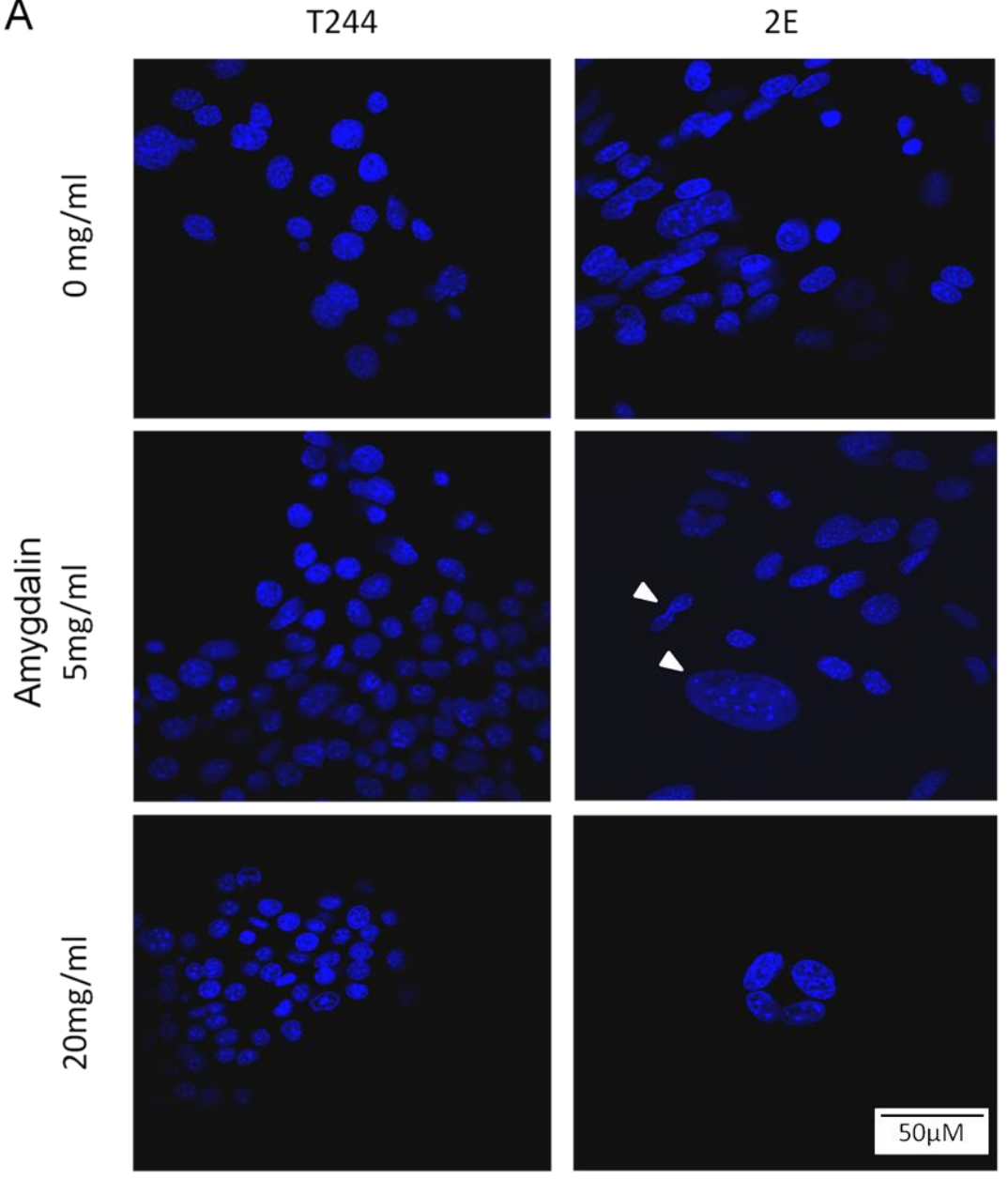

B

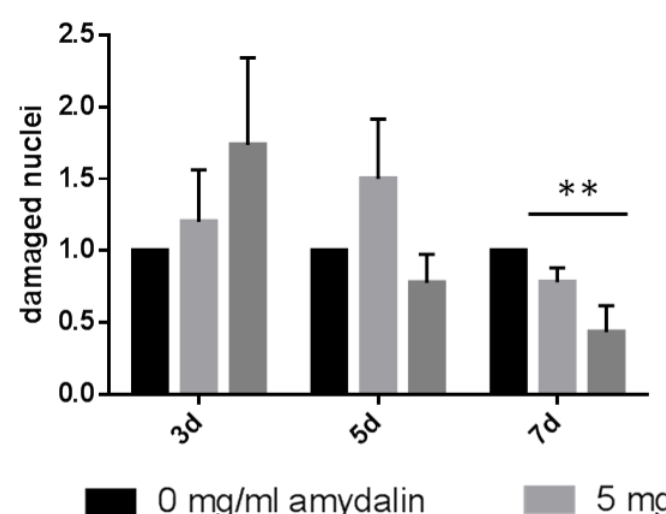

C

$2 E$

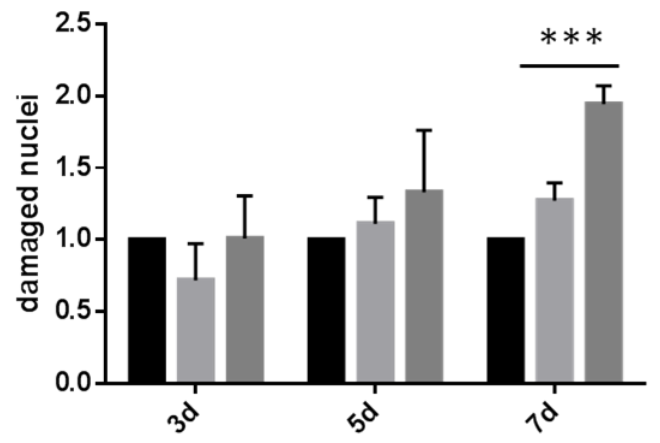

$5 \mathrm{mg} / \mathrm{ml}$ amygdalin

$20 \mathrm{mg} / \mathrm{ml}$ amygdalin

Figure 3.53: Mitotic catastrophe in primary PCa cell lines T244 and 2E after amygdalin treatment. (A) T244 and 2E cells were treated with $0 \mathrm{mg} / \mathrm{ml}, 5 \mathrm{mg} / \mathrm{ml}$ or $20 \mathrm{mg} / \mathrm{ml}$ amygdalin for $3 \mathrm{~d}$, $5 \mathrm{~d}$ or $7 \mathrm{~d}$ and damaged nuclei were counted in five randomly taken pictures using the fluorescent microscope. After $7 \mathrm{~d}$ treatment with $20 \mathrm{mg} / \mathrm{ml}$ amygdalin (B) reduced mitotic catastrophe in T244 cells by $53 \%$ was detected and (C) 2 -fold increased mitotic catastrophe in $2 \mathrm{E}$ cells was observed. The data represent the mean \pm s.d. of three independent experiments. Significance was calculated using the student's t-test. ${ }^{*} p<0.05,{ }^{* *} p<0.01,{ }^{* * *} p<0.0001$ compared with control. 


\section{Discussion}

\subsection{Summary of results}

The two main aims of this study were the analysis of the role of estrogen receptors (ERs) in prostate cancer (PCa) and $\mathrm{PCa}$ treatment and to study the therapeutic potential of the alternative cancer drug amygdalin for PCa treatment.

The role of ERs in PCa was analyzed in ER a knockout (ERKO)/TRAMP or ER $\beta$ knockout (BERKO)/TRAMP mice. Survival time, tumor incidence and signaling in primary cell culture should be analyzed. The mean survival of BERKO/TRAMP and TRAMP mice did not differ from each other, whereas survival of ERKO/TRAMP mice compared to TRAMP mice was significantly reduced. ERKO/TRAMP mice developed PIN lesions and extensive seminal gland cancer (SGCa) but no PCa during this study. Tumor incidence and malignancy (progression stage) did not differ between TRAMP and BERKO/TRAMP mice. One TRAMP (T244 cells) and one BERKO/TRAMP (BERKO/TRAMP 283 cells) cell line was established from $\mathrm{PCa}$, respectively. Although genotyping $\mathrm{PCR}$ and sequence analysis confirmed a BERKO genotype of BERKO/TRAMP 283 cells, western blot analysis still showed strong expression of ER $\beta$. T244 and BERKO/TRAMP 283 cells showed no differences in cell proliferation. The ambiguous expression of ER $\beta$ in the murine primary PCa cell lines impeded conclusions about the role of ER $\beta$ in PCa development.

In order to investigate the therapeutic potential of ER $\beta$ activation on different stages of $\mathrm{PCa}$, a cell system derived by Priv. Doz. Dr. Paul Thelen was used. The cell system was composed of three variants of the human PCa cell line VCaP. Based on the castrationresistant $\mathrm{PCa}$ (CRPC) cell line VCaP, VCaP rev. cells were generated by treatment of VCaP cells with testosterone for seven month. VCaP AA cells were generated by treatment of VCaP cells with the agent abiraterone for at least three weeks until the cells developed a resistance against abiraterone. To characterize the cell model, the expression of PCarelevant genes, i.e. AR and PSA in the three VCaP cell variants was examined. VCaP rev. cells represented early PCa stages. Compared to the other VCaP cell variants VCaP rev. cells showed low AR expression and lower expression of the PCa marker PSA. VCaP cells represented an advanced PCa stage, already gaining adaptation to ADT. VCaP cells showed high AR expression and moderate PSA expression. VCaP AA cells represented the most advanced PCa stage, with resistance to androgen deprivation therapy (ADT), high AR and PSA expression and expression of constitutively active androgen receptor splice variants (ARVs).

This cell model was used to study the activation of ER $\beta$ by treating the VCaP cell variants with the ER $\beta$ agonist $8 \beta$-VE2. Next, cell survival, apoptosis induction and gene expression were analyzed. Concentrations of $25 \mu \mathrm{M} 8 \beta$-VE2-induced cell survival reduction and apoptosis in all VCaP cell variants. In VCaP rev. cells, the AR expression was increased 
upon $8 \beta$-VE2 treatment but AR-regulated gene expression was decreased. In VCaP and VCaP AA cells $8 \beta$-VE2 treatment reduced AR expression but increased AR-regulated gene expression. Of note, in VCaP AA cells the expression of $A R V s$ was remarkable downregulated after $8 \beta$-VE2 treatment.

A whole mRNA-sequencing analysis was performed on VCaP cell variants before and after treatment with $8 \beta-V E 2$ to elucidate the molecular mechanisms underlying these effects. Surprisingly, no evidence for ER $\beta$-mediated signaling and only minor expression of ER $\beta$ mRNA could be found in whole mRNA-sequencing data. Indeed, the knowledge about ER $\beta$ signaling in $\mathrm{PCa}$ is limited. Therefore, further experiments concerning the role of ER $\beta$ in $8 \beta$ VE2 mediated treatment effects were performed. Two different ER $\beta$ agonists, ERB 041 and FERb 033, could not reproduce the effects on survival, apoptosis and gene expression observed after $8 \beta$-VE2 treatment in VCaP cells. Furthermore, the effects of $8 \beta$-VE2 treatment in VCaP cells could not be inhibited by blocking ER $\beta$ with the antagonist fulvestrant.

To further elucidate the molecular processes in $8 \beta$-VE2-treated VCaP variants, whole mRNA-sequencing data were screened for candidate genes pointing to pathways connected to PCa. The cholesterol synthesis pathway and PI3K pathway could be excluded as the underlying cause for $8 \beta$-VE2-mediated effects. The induction of AR-regulated gene expression, further consolidated by whole mRNA-sequencing data, point to an involvement of $A R$ signaling. AR expression as well as expression of constitutively active AR splice variants was downregulated upon $8 \beta$-VE2 treatment and therefore excludes these two escape mechanisms as the underlying cause for AR activation. Furthermore, mRNA analysis could exclude the overexpression of glucocorticoid receptor or intratumoral testosterone synthesis as underlying cause for induction of AR target gene expression after 8 $\beta$-VE2 treatment. The aim to inhibit AR in VCaP cells showed that the antiandrogen bicalutamide is converted into an AR agonist in VCaP cells. Further experiments demonstrated that the AR ligand DHT induced similar gene expression as observed in 8 $\beta$-VE2-treated VCaP cells and also induced early cell survival reduction, but could not induce apoptosis. Treatment of the AR-negative human PCa cell line PC3 with 8 $\beta$-VE2 did not induce reduction of cell survival or induction of apoptosis. A reporter gene analysis with ER- and AR-specific reporter genes did not show any transcriptional activity of $\mathrm{ER}$ in $\mathrm{VCaP}$ cells and only minor activation of $\mathrm{AR}$ transcriptional activity upon $8 \beta$-VE2 treatment.

In summary, our results demonstrated that $8 \beta$-VE2 treatment reduced cell survival and induced apoptosis in human VCaP cell variants and that these cellular events occur either in an ER $\beta$-independent manner or in an ER $\beta$-dependent mechanism which is not known to date. The expression of $A R$ and ARVs was downregulated upon $8 \beta$-VE2 treatment, but expression of several AR-regulated genes was induced. However, how these effects are mediated at the molecular level could not be fully elucidated. 
The second aim of the study was the analysis of the therapeutic potential of the alternative cancer drug amygdalin for PCa treatment. TRAMP-FVB mice were treated with five i.p. injections weekly of $10 \mathrm{mg} / \mathrm{kg}, 100 \mathrm{mg} / \mathrm{kg}$ or $100 \mathrm{mg} / \mathrm{kg}$ purified amygdalin. For preventive treatment mice were treated from the age of six weeks on, for curative treatment amygdalin injections were started at the age of 16 weeks. No differences in weight gain, no toxic effects on liver, lung, kidney, spleen or the urogenital tract were detectable in treated and control animals. No differences in mean survival time were observed. No signs of cyanide or thiocyanate were detected in blood or serum of amygdalin-treated mice. Preventive treatment with $100 \mathrm{mg} / \mathrm{kg}$ amygdalin decreased metastasis formation compared to untreated mice. Analysis of induction of cell signaling upon amygdalin treatment failed due to massive induction of necrosis in TRAMP PCa. Indeed, mice treated with $100 \mathrm{mg} / \mathrm{kg}$ amygdalin showed significantly higher percentage of necrotic area. Since validity of experiments in vivo was limited by the massive occurrence of necrotic tissue, analysis of cell signaling was proceeded in vitro using primary murine PCa cell lines T244 and 2E. Cell number and proliferation rate were reduced by treatment with amygdalin, but neither cytotoxic effects nor apoptosis were observed. Protein analysis revealed that similar to in vivo experiments proteins obtained from in vitro experiments were degraded. Long term treatment of primary murine PCa cell lines with amygdalin led to cell morphological changes. The usually round or elongated T244 and 2E cells showed spine formation or a flatted, undefined shape. The induction of senescence was identified as the underlying cause for the cell shape changes as well as the reduced cell number. T244 as well as 2E cells induced SA $\beta$-gal expression after $7 \mathrm{~d}$ of amygdalin treatment. The induction of senescence can be the reason of different cellular events, e.g. double strand breaks (DSB) and mitotic catastrophe. Here, DSB were induced in both PCa cell lines upon amygdalin treatment. Additionally, in amygdalin-treated $2 \mathrm{E}$ cells also induction of mitotic catastrophe was observed.

Taken together, the in vivo analysis of the effect of amygdalin on PCa growth in TRAMP-FVB mice did not show increased survival or reduction of $\mathrm{PCa}$ incidence. However, preventive treatment with $100 \mathrm{mg} / \mathrm{kg}$ amygdalin decreased metastasis formation in TRAMP-FVB mice and treatment with $100 \mathrm{mg} / \mathrm{kg}$ amygdalin also induced an increase of necrosis formation. Finally, in vitro experiments performed with different PCa cell lines identified senescence induction as the amygdalin-mediated cellular mechanism of action.

\subsection{Are the used ER mouse models suitable for knockout experiments?}

To date, different in vivo approaches were performed to elucidate the role of ERs in PCa. The two types of the ER, namely ER $\alpha$ and ER $\beta$, were described to play a role in prostate and PCa formation. Whereas ER $\alpha$ is described to be mainly expressed in the stromal 
compartment of the prostate, ER $\beta$ is mainly expressed in basal-epithelial prostate cells (Leav et al. 2001; Royuela et al. 2001). Neonatal or long term exposure to estrogens together with testosterone leads in $100 \%$ of cases to a development of PCa in rats (Bosland et al. 1995). Studies using ER $\alpha$ knockout (ERKO) and ER $\beta$ knockout (BERKO) mice showed that this estrogen/testosterone-induced carcinogenesis is mediated by the ER $\alpha$ (Ricke et al. 2008). Another study by Wang et al. (2004) described that ER a expression is increased in TRAMP mice prostate compared to wild type prostate, further supporting the evidence that ER a exhibits PCa promoting function. Furthermore, studies performed by McPherson et al. (2007), using aromatase-deficient mice that lack the capability to produce estrogens, showed that ER $\beta$ but not ER $\alpha$ activation suppresses development of prostatic hyperplasia. Further evidence for the role of ER $\beta$ in prostate cancer was described by Horvath et al. (2001). These authors analyzed the expression of $E R \beta$ in five normal prostates and 159 specimens from radical prostatectomies. Horvath et al. (2001) identified high ER $\beta$ expression in $100 \%$ of normal prostate and declining ER $\beta$ expression in hyperplasic prostate tissue (24.2\% ER $\beta$-positive) and carcinomas (11.3\% ER $\beta$-positive). Furthermore, ER $\beta$ expression was found to be associated with a longer relapse-free survival (Horvath et al. 2001). Taken together, these data indicate that ER $\alpha$ rather promotes PCa development, whereas ER $\beta$ might act as a tumor suppressor in $\mathrm{PCa}$.

In the present study, ERKO/TRAMP and BERKO/TRAMP mouse models previously described by Slusarz et al. (2012) were used to investigate the role of ERs in PCa. The TRAMP (transgenic adenocarcinoma of mouse prostate) mouse model expresses the simian virus 40 (SV40) T/t antigens under the control of the prostate-specific probasin promoter. T/t antigens are dominant active oncoproteins, inactivating the tumor suppressor proteins retinoblastoma (pRb) and p53 (Ahuja et al. 2005). Due to the SV40 T/t expression, PCa development is induced. In the present study one of the major aims were to investigate how the knockout of ER $\alpha$ or ER $\beta$ could influence prostate cancer development and progression in ERKO/TRAMP and BERKO/TRAMP mice. For further investigations primary PCa cell lines should be generated from tumors of these mice in order to elucidate cellular ER signaling. Here, it was observed that ERKO/TRAMP mice developed PIN lesions and massive SGCa. The incidence of SGCa was not increased in ERKO/TRAMP mice compared to TRAMP and BERKO/TRAMP mice, but the SGCa growth proceeded faster and mice had to be sacrificed earlier. The data collected in this study correspond with the current knowledge concerning the growth promoting role of ER $\alpha$ in PCa (Ricke et al. 2008; McPherson et al. 2007). However, no long term studies could be performed using the ERKO/TRAMP model because the accelerated growth of SGCa constrained early termination of the experiments. The development of SGCa in TRAMP mice is a well-known disadvantage of the TRAMP mouse model since SGCa do only seldom occur in humans (Tani et al. 2005; Tarjan et al. 2009). However, most studies using TRAMP mice did not comment on SGCa growth, i.e. Slusarz et 
al. (2012). It remains unclear why SGCa growth is accelerated in ERKO/TRAMP mice. Comparing the data of the present study to the data presented by Slusarz et al. (2012), it is apparent that classification of PCa stage used by Slusarz et al. (2012) differs from the classification used in the present study. Slusarz et al. performed all experiments with 5month old mice and differentiated between non-cancerous stages (normal tissue, hyperplasic tissue and PIN lesions) and cancerous stages (well-differentiated carcinoma (WDC), moderate-differentiated carcinoma (MDC) and poorly-differentiated carcinoma (PDC)). MDC were only described in few cases and were not further described by Slusarz et al., i.e. by a representative picture. The current study differentiated between normal prostate tissue (which was not observed in any mouse with a TRAMP genotype), PIN lesions, PCa and metastatic PCa. Furthermore, the present study analyzed the incidence of metastatic PCa or SGCa, which were both not described by Slusarz et al. (2012). However, reduced PCa incidence in ERKO/TRAMP mice observed in the present study was also shown by Slusarz et al. (2012), i.e. these authors found a reduction of PDC incidence in ERKO/TRAMP by $75 \%$ compared to TRAMP mice. In contrast to the present study, Slusarz et al. (2012) reported a reduced incidence of PIN lesions in ERKO/TRAMP mice compared to TRAMP mice (only $5 \%$ in ERKO/TRAMP mice compared to $23 \%$ in TRAMP mice) and an increased incidence of WDC (85\% in ERKO/TRAMP compared to $50 \%$ in TRAMP mice) (see Tab. 4.1). However, comparing the representative tissue sections shown by Slusarz et al. (2012) for PIN lesions, WDC and PDC to the criteria used in the present study for PIN lesions, PCa and metastatic $\mathrm{PCa}$, it became clear that a comparison between the data could be misleading. The representative pictures of WDCs shown in Slusarz et al. (2012) would have been classified as PIN lesions in the present study. Therefore, a comparison between the present study and Slusarz et al (2012) is limited by the differently chosen classification of tumor sections. In the present study ERKO/TRAMP mice developed only PIN, but progression to PCa was not observed, thus, primary cell cultures could not be established to further investigate the molecular mechanisms behind the ER a knockout.

In contrast to Slusarz et al. (2012), the present study could not show a tumor suppressive function of ER $\beta$ in PCa. The BERKO/TRAMP mice developed PCa, but the occurrence of $\mathrm{PCa}$ in BERKO/TRAMP compared to TRAMP mice was not increased. Slusarz et al. (2012) described that twice as much PDCs were found in BERKO/TRAMP compared to TRAMP mice. Furthermore Slusarz et al. (2012) described a $15 \%$ reduced incidence of WDC compared to TRAMP mice. In the present study, an increase of tumor incidence and evidence for increased tumor aggressiveness was not observed, e.g. increased metastasis formation could also not be detected in BERKO/TRAMP PCa compared to TRAMP PCa (see Tab. 4.1).

Moreover, in vitro analysis in the present study did not reveal increased proliferation rate in BERKO/TRAMP murine primary PCa cells compared to TRAMP murine primary PCa cells. 
Table 4.1: Development of PCa in TRAMP, ERKO/TRAMP and BERKO/TRAMP mice observed in the present study compared to Slusarz et al. (2012). Listed are the incidences and progression stages of PCa in TRAMP, ERKO/TRAMP and BERKO/TRAMP mice observed in the present study and by Slusarz et al. (2012). PIN: prostatic intraepithelial neoplasia, WDP: well-differentiated PCa, PDC: poorly-differentiated PCa

\begin{tabular}{|c|c|c|c|c|c|c|}
\hline & \multicolumn{3}{|c|}{ The present study } & \multicolumn{3}{|c|}{ Slusarz et al. (2012) } \\
\hline Mouse strain & PIN & $\mathrm{PCa}$ & $\begin{array}{c}\text { metastatic } \\
\mathrm{PCa}\end{array}$ & PIN & WDC & PDC \\
\hline TRAMP & $56 \%$ & $17 \%$ & $21 \%$ & $23 \%$ & $50 \%$ & $20 \%$ \\
\hline ERKO/TRAMP & $100 \%$ & $0 \%$ & $0 \%$ & $5 \%$ & $85 \%$ & $5 \%$ \\
\hline BERKO/TRAMP & $70 \%$ & $15 \%$ & $15 \%$ & $26 \%$ & $35 \%$ & $39 \%$ \\
\hline
\end{tabular}

However, in the present study it could not be definitely proven that the BERKO mice indeed did not express ER $\beta$. The genotyping PCR as well as the sequencing analysis confirmed the BERKO genotype of the used mouse model. Nonetheless, protein analysis detected ER $\beta$ protein expression in kidney of BERKO/TRAMP mice, liver, testis and prostate of TRAMPFVB mice, human VCaP cells, primary, murine PCa cell line T244 and mouse brain. The ER $\beta$ expression was diminished by an ER $\beta$-specific blocking peptide. Two possible explanations for this phenomenon seem to be reasonable. First, the observed expression of ER $\beta$ protein is an artifact which is attributable to a non-specific binding of the ER $\beta$ antibodies used in the western blot experiments. Variable specificity of ER $\beta$ antibodies was already discussed in a recent study (Skliris et al. 2002). Skliris and coworkers examined the specificity of seven ER $\beta$-specific antibodies on human breast cancer samples. They found that specificity is strongly dependent on the used method (immunohistochemistry, western blot and flow cytometry) and differs strongly between the tested antibodies. Therefore, specificity of ER $\beta$-detecting antibodies constitutes a major problem in research. Assuming that the lack of antibody specificity leads to the detection of an unspecific signal, the mouse model used in the present study exhibits indeed an ER $\beta$ knockout and could therefore not support the described tumor suppressive function of ER $\beta$ in PCa (Horvath et al. 2001; Hurtado et al. 2008; Dey et al. 2014). Indeed, the study by Skliris et al. did not describe the ER $\beta$ antibodies used in the present experiments and ER $\beta$ antibody specificity might have improved since 2002. In the present work detection of ER $\beta$ expression in different tissues was reproducible with different ER $\beta$ antibodies and diminished by co-incubation with an ER $\beta$-specific peptide. These results indicate that the ER $\beta$ antibody is specific. Interestingly, Slusarz et al. (2012) did not provide results using western blot analysis in their study to validate the ER $\beta$ knockout in BERKO/TRAMP mice, but immunohistochemical staining was performed on tissue sections to prove the ER $\beta$ knockout. 
A second explanation for the discrepancies observed in the present BERKO/TRAMP mouse model could be that the knockout of ER $\beta$ in the used mouse model was not quite complete. Although sequencing analysis confirmed that sequence aberrations did not occur in the respective knockout cassette, the possibility of alternative splicing cannot be ruled out entirely. The knockout cassette is located in exon 3 of the mouse Esr2 (ER $\beta$ ) gene (Krege et al. 1998), however, an ER $\beta$ splice variant with a deletion of exon 3 was already described (ER $\beta \Delta$ ex3) (Fig. 4.1). Exon 3 of the mouse Esr2 gene encodes for the DNA binding domain of the ER $\beta$ and therefore is essential for the transcriptional activity of the ER $\beta$ (Price, JR et al. 2001). Only recently Maneix et al. (2015) described that mice with expression of ER $\beta \Delta$ ex3 showed only mild impairments compared to a full ER $\beta$ knockout. They conclude that several functions of ER $\beta$ are independent of the transcriptional activity of the receptor. Thus, it can be assumed that the expression of ER $\beta \Delta \operatorname{ex} 3$ is responsible for the observed ER $\beta$ signal in the present western blot experiments which in turn leads to the lack of evidence of ER $\beta$-mediated tumor suppressive function in the present study.
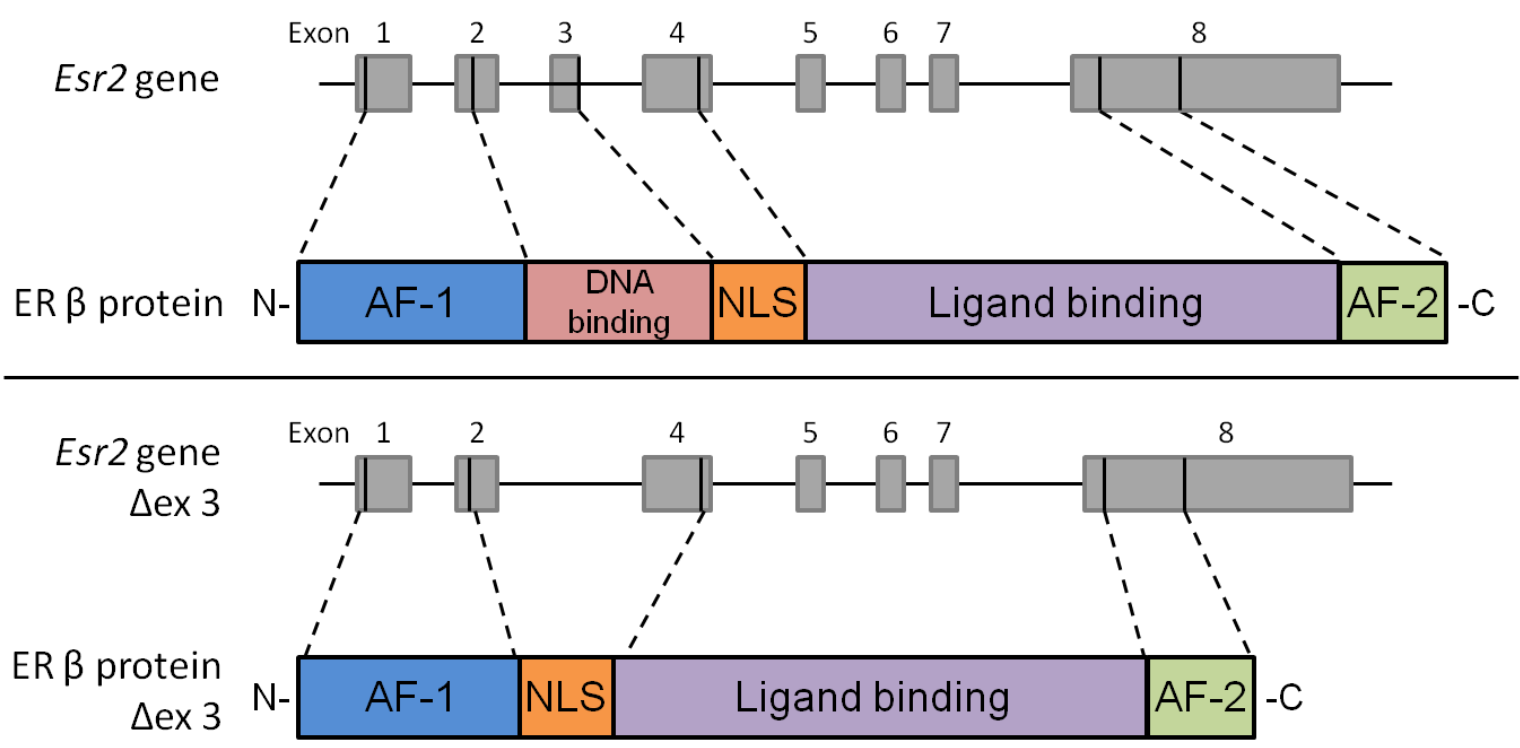

Figure 4.1: Structure of the wild type and $\Delta$ ex3 Esr2 gene and protein. Depicted is a schematic illustration of the wild type Esr2 gene and protein (upper figure) and the $\Delta$ ex3 Esr2 gene and protein described by Maneix et al (2015) (lower figure). The deletion of exon 3 results in the generation of an ER $\beta$ protein lacking the DNA binding domain. We hypothesized that the BERKO mouse model used in the present study expresses a comparable ER $\beta$ splice variant, lacking exon 3 and therefore also lacking the knockout cassette. Modified according to Maneix et al. (2015) and Smart et al. (2013).

The role of human ER $\beta$ splice variants was described by different studies and it was suggested that in contrast to full length $E R \beta, E R \beta$ splice variants exhibit rather tumor promoting than tumor suppressive functions in LNCaP and PC3 PCa cell lines (Leung et al. 2010; Dey et al. 2012; Hurtado et al. 2008). Especially the splice variants ER $\beta 2$ and ER $\beta 5$ are suspected to exhibit tumor promoting functions. Both ER $\beta$ splice variants lack the AF-2 domain and differ in the LBD from full length ER $\beta$ (Christoforou et al. 2014). In contrast to 
human $E R \beta$, only little is known about the role of murine $E R \beta$ splice variants in PCa. One study by Lu et al. (1998) suggested that human and murine ER $\beta$ splice variants might not equal each other. However, to date, tumor promoting function could not be associated with the murine ER $\beta$ splice variant ER $\beta \Delta$ ex3 (Maneix et al. 2015).

Taken together, the in vivo and in vitro results of the present study indicate that the chosen mouse models were not quite suitable for the molecular analysis of the role of ER $\alpha$ or ER $\beta$ in PCa development and progression. On the one hand, ERKO/TRAMP mice only developed PIN lesions in the prostate, but these premalignant lesions did not further progress to PCa, and therefore primary cell cultures could not be established. On the other hand, BERKO/TRAMP mice did not show the expected phenotype with increased aggressive PCa development and moreover, could not be clearly identified to be completely ER $\beta$-negative.

\subsection{Treatment of PCa cells with the ER $\boldsymbol{\beta}$ agonist $8 \beta$-VE2}

The ER $\beta$ is suggested to act as a tumor suppressor in PCa (Cheng et al. 2004; Hurtado et al. 2008; Dey et al. 2014) and activation of ER $\beta$ in PCa cells results in induction of apoptosis and cell cycle arrest (Hurtado et al. 2008; Nakamura et al. 2013; Cheng et al. 2004). Here, the effect of the synthetic steroidal estrogen $8 \beta$-VE2 on different PCa cell stages was analyzed in vitro. 8$\beta$-VE2 (8-vinylestra-1,3,5 (10)-triene-3,17 $\beta$-diol) is an ER $\beta$-specific agonist based on the structural form of estradiol with an additional vinyl-group at the $8 \beta$ position (Hillisch et al. 2004, 2004) (Fig. 4.2). The vinyl-group designates the specificity of $8 \beta$-VE2 to ER $\beta$. The binding selectivity of $8 \beta$-VE2 is 183 -fold higher to ER $\beta$ than to ER $\alpha$ (Hillisch et al. 2004).

E2

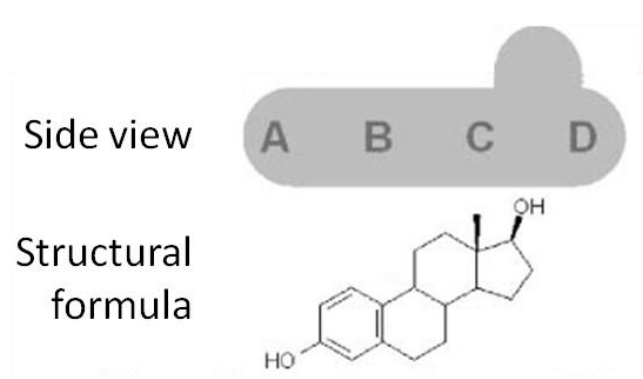

$8 \beta-V E 2$

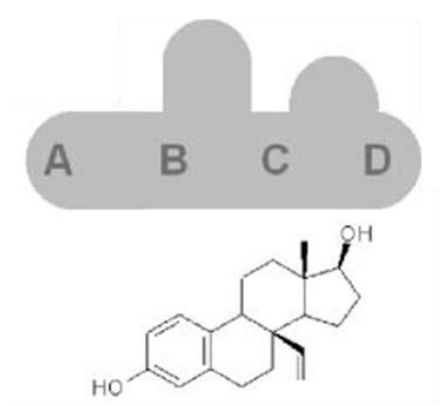

Figure 4.2: Structural comparison of estradiol (E2) and $\mathbf{8} \beta$-VE2. The ER $\beta$ agonist $8 \beta$-VE2 was designed based on the structural formula of the endogenous ER ligand estradiol. The addition of a vinyl-group at the $8 \beta$ position determined the specific binding to ER $\beta$. Modified according to Hillisch et al. (2004).

The present study aimed to reveal the effect of ER $\beta$ activation on a cellular model representing the clinical cause of PCa progression. The VCaP cell variants, VCaP rev., $\mathrm{VCaP}$ and VCaP AA mimic the different stages of PCa progression. VCaP cells exhibit an 
AR overexpression as adaptation to ADT which is the most commonly observed adaptation in patients (Linja et al. 2001; Ford et al. 2003). VCaP rev. cells mimic early PCa before ADT, VCaP cells represent advanced PCa with adaptation to ADT and VCaP AA cells mimic advanced PCa under maximum ADT. This study showed that treatment with $8 \beta$-VE2 reduced cell survival and induced apoptosis in all PCa stages (VCaP rev., VCaP and VCaP AA). Apoptosis describes the programmed cell death. Here, the generation of apoptotic bodies that are phagocytosed by lymphocytes ensures recycling of cell components. Apoptosis can be induced via two major pathways, i.e. extrinsic and intrinsic apoptosis, both inducing the activation of caspases (cysteine-dependent aspartate-directed proteases). Furthermore, apoptosis is an ATP-dependent pathway (Elmore 2007). Induction of apoptosis upon 8ß-VE2 treatment in PCa cell lines was previously described in different studies. McPherson et al. (2010) stated that the ER $\beta$ activation induced androgen-independent apoptosis in PCa cell line PC3. Hussain et al. (2012) found apoptosis induced by $8 \beta$-VE2 in quiescent castrationresistant stem cells. Dey et al. (2014) described 8 $\beta$-VE2-induced apoptosis in ER $\beta$ overexpressing androgen-dependent (LNCaP) and androgen-independent (PC3) PCa cell lines. To date, however, little is known about the molecular signaling that induces apoptosis upon ER $\beta$ activation. McPherson et al. (2010) found TNF a signaling as an essential pathway for ER $\beta$-induced apoptosis and these authors claimed that ER $\beta$-induced apoptosis is mediated via the extrinsic apoptotic pathway. In contrast to these findings Dey et al. (2014) reported that ER $\beta$-induced apoptosis is mediated via PUMA ( $p 53$ upregulated modulator of apoptosis) and triggered by the intrinsic apoptotic pathway. Taken together, reports in the literature indicate that $8 \beta-\mathrm{VE} 2$ is a potent ER $\beta$ agonist, feasible for treatment of PCa cells, independent of AR expression and even suitable for castration-resistant PCa cells.

The present study showed that the treatment of the cell variants VCaP and VCaP AA with $8 \beta$-VE2 reduced the expression of $A R$ and its splice variants, but induced AR-mediated gene expression (see 3.1.2.2 and 3.1.2.3). This observation is surprising, since the downregulation of AR gene expression should coincide with a decreased expression of its target genes (Lund et al. 2004; Thelen et al. 2007). To date, no other working group described the activation of $A R$ transcriptional activity in PCa cells after $8 \beta$-VE2 treatment and only one other agent was reported to induce comparable effects in PCa cells (apoptosis, AR downregulation and induction of AR target genes). This agent, namely HE3235 (17a-ethynyl-

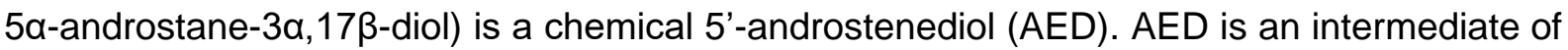
steroid synthesis and the exact function in PCa is still under debate (Miyamoto et al. 1998; Maggiolini et al. 2004). Interestingly, AEDs showed the ability to transactivate AR and ER in PCa (Miyamoto et al. 1998). However, HE3235 is described as an ER $\beta$ agonist with antiandrogenic functions (Trauger et al. 2009; Ahlem et al. 2012). HE3235 induced apoptosis in AR-positive PCa cells in vitro and in vivo, but did not affect AR-negative cell lines (Trauger et al. 2009; Koreckij et al. 2009). This indicates that HE3235 induces apoptosis in an 
androgen-dependent manner. However, clinical pretrials testing HE3235 for the use of PCa treatment were stopped for unknown reasons.

We analyzed the 10 most up- or downregulated genes after $8 \beta$-VE2 treatment in VCaP cells and their function in PCa. 4 of 10 upregulated genes (ORM2, CACNG4, ENDOD1, FAM189A2) and 8 of 10 downregulated genes (ERP27, ASZ1, ADGRF1, TRPM3, ADGRF5, VWA5B1, SLC3A1 and LRRN1) had no known function in PCa (see Tab. 4.2). The expression of 3 of 10 upregulated genes (TRPM8, AGR2, INSIG1) is known to be induced by AR signaling and 3 of 10 downregulated genes (ERP27, ASZ1, LRRN1) are known to be repressed by $A R$ signaling. Two of the upregulated genes (STEAP4, SLC45A3) were reported to have tumor growth suppressive functions (Tamura, Chiba 2009; Perner et al. 2013) and the downregulated PCAT1 was described to be an important regulator of PCa cell progression (Prensner et al. 2014a; Prensner et al. 2014b; Prensner et al. 2011). However, also B3GNT6 downregulation was observed which was described to potentially increase PCa cell invasiveness (Lee et al. 2009). In summary, the most up- and downregulated genes after $8 \beta$-VE2 treatment in VCaP cells could not elucidate the underlying mechanism but showed that tumor suppressive gene expression was activated and oncogenic gene expression was downregulated after $8 \beta$-VE2 treatment in $\mathrm{VCaP}$ cells. Interestingly, 6 genes known to be regulated by AR signaling are among the most differentially expressed candidate genes.

Table 4.2: Overview over the 10 most up- and downregulated genes upon $8 \beta-V E 2$ treatment in VCaP cells. Listed are the 10 most up- and downregulated genes regulated by $8 \beta$-VE2 in VCaP cells indentified in the whole mRNA-sequencing analysis and their function in PCa.

Most upregulated genes upon $8 \beta-V E 2$ treatment

\begin{tabular}{l|l|l}
\hline Gene symbol & Gene name & Function in PCa \\
\hline \multirow{2}{*}{ STEAP4 } & STEAP family member 4 & $\begin{array}{l}\text { STEAP4 expression regulates focal adhesion } \\
\text { kinase activation in the human androgen- } \\
\text { independent prostate cancer cells DU145 PCa } \\
\text { and anchorage-independent cell growth, } \\
\text { meaning STEAP4 expression reduces cell } \\
\text { invasiveness (Tamura, Chiba 2009). }\end{array}$ \\
\hline \multirow{5}{*}{ SLC2A3 } & $\begin{array}{l}\text { solute carrier family } \\
\text { (facilitated } \\
\text { transporter), member 3 }\end{array}$ & $\begin{array}{l}\text { SLC2A3 is involved in the glycolytic } \\
\text { metabolism. The glycolytic metabolism was } \\
\text { found to be differentially expressed in } \\
\text { androgen-responsive PCa cells (LNCaP) and } \\
\text { nonresponsive PCa cells (PC3). However, the } \\
\text { effect of differentially expression on PCa is } \\
\text { unknown (Vaz et al. 2012) }\end{array}$ \\
\hline
\end{tabular}




\begin{tabular}{|c|c|c|}
\hline SLC45A3 & $\begin{array}{l}\text { solute carrier family } 45 \text {, } \\
\text { member } 3\end{array}$ & $\begin{array}{l}\text { Loss of SLC45A3 protein expression in } \\
\text { prostate cancer is associated with SLC45A3- } \\
\text { ERG gene rearrangement and an unfavorable } \\
\text { clinical course (Perner et al. 2013). }\end{array}$ \\
\hline TRPM8 & $\begin{array}{l}\text { transient receptor potential } \\
\text { cation channel, subfamily } \\
\text { M, member } 8\end{array}$ & $\begin{array}{l}\text { The expression of TRPM8 is androgen- } \\
\text { regulated. TRPM8 possibly serves as a } \\
\text { testosterone receptor in PCa (Asuthkar et al. } \\
2015 \text { ) and serves as a potential diagnostic } \\
\text { marker for PCa (Zhang, Barritt 2006). }\end{array}$ \\
\hline AGR2 & anterior gradient 2 & $\begin{array}{l}\text { The expression of AGR2 is androgen-regulated } \\
\text { and serves as a potential diagnostic marker for } \\
\text { PCa (Waltering et al. 2009; Bu et al. 2011). A } \\
\text { knockdown of AGR2 was associated with the } \\
\text { induction of senescence in PCa cell lines } \\
\text { LNCaP and DU145 (Hu et al. 2012). }\end{array}$ \\
\hline INSIG1 & insulin induced gene 1 & $\begin{array}{l}\text { Expression of INSIG1 is AR-regulated } \\
\text { (Waltering et al. 2009). INSIG1 regulates } \\
\text { cholesterol concentrations and is } \\
\text { overexpressed in PCa (Sharad et al. 2011). }\end{array}$ \\
\hline ORM2 & orosomucoid 2 & No connection to PCa known \\
\hline CACNG4 & $\begin{array}{l}\text { calcium channel, voltage- } \\
\text { dependent, } \\
\text { subunit } 4\end{array}$ & No connection to PCa known \\
\hline ENDOD1 & $\begin{array}{l}\text { endonuclease domain } \\
\text { containing } 1\end{array}$ & No connection to PCa known \\
\hline FAM189A2 & $\begin{array}{l}\text { family with sequence } \\
\text { similarity } 189, \text { member A2 }\end{array}$ & No connection to PCa known \\
\hline
\end{tabular}

Most downregulated genes upon $8 \beta-V E 2$ treatment

\begin{tabular}{l|l|l}
\hline Gene symbol & Gene name & Function in PCa \\
\hline ERP27 & $\begin{array}{l}\text { endoplasmic reticulum } \\
\text { protein 27 }\end{array}$ & $\begin{array}{l}\text { For ERP27 expression no connection to PCa is } \\
\text { known but the expression of ERP27 is AR- } \\
\text { repressed (Waltering et al. 2009) }\end{array}$ \\
\hline ADGRF1 & $\begin{array}{l}\text { adhesion G protein- } \\
\text { coupled receptor F1 }\end{array}$ & No connection to PCa known \\
\hline
\end{tabular}




\begin{tabular}{|c|c|c|}
\hline$A S Z 1$ & $\begin{array}{l}\text { ankyrin repeat, SAM and } \\
\text { basic leucine zipper } \\
\text { domain containing } 1\end{array}$ & $\begin{array}{l}\text { ASZ1 expression was not connected to PCa so } \\
\text { far, but the expression of ASZ1 is AR- } \\
\text { repressed (Waltering et al. 2009) }\end{array}$ \\
\hline TRPMЗ & $\begin{array}{l}\text { transient receptor potential } \\
\text { cation channel, subfamily } \\
\text { M, member } 3\end{array}$ & No connection to PCa known \\
\hline ADGRF5 & $\begin{array}{l}\text { adhesion G protein- } \\
\text { coupled receptor F5 }\end{array}$ & No connection to PCa known \\
\hline VWA5B1 & $\begin{array}{l}\text { von Willebrand factor } \mathrm{A} \\
\text { domain containing 5B1 }\end{array}$ & No connection to PCa known \\
\hline B3GNT6 & $\begin{array}{l}\text { UDP-GIcNAc:betaGal } \\
\text { beta-1,3-N-acetyl } \\
\text { glucosaminyltransferase } 6\end{array}$ & $\begin{array}{l}\text { B3GNT6 expression suppresses PCa cell } \\
\text { invasive potential in LNCaP and PC3 cells (Lee } \\
\text { et al. 2009) }\end{array}$ \\
\hline LRRN1 & $\begin{array}{l}\text { leucine rich repeat } \\
\text { neuronal } 1\end{array}$ & $\begin{array}{l}\text { Expression of LRRN1 is strongly repressed by } \\
\text { AR signaling (Wu et al. 2014). However, the } \\
\text { function of LRRN1 in } \mathrm{PCa} \text { is unknown. }\end{array}$ \\
\hline SLC3A1 & $\begin{array}{l}\text { solute carrier family } 3 \\
\text { (amino acid transporter } \\
\text { heavy chain), member } 1\end{array}$ & No connection to PCa known \\
\hline PCAT1 & $\begin{array}{l}\text { prostate cancer } \\
\text { associated transcript } \\
\text { (non-protein coding) }\end{array}$ & $\begin{array}{l}\text { PCAT-1 is a long non-coding RNA expressed } \\
\text { in PCa (Prensner et al. 2011). PCAT-1 was } \\
\text { associated with gene expression promoting } \\
\text { PCa progression (Prensner et al. 2014a). } \\
\text { PCAT-1 is described to promote chromosomal } \\
\text { instability and stabilizes the oncogene cMyc in } \\
\text { PCa (Prensner et al. 2014a; Prensner et al. } \\
2014 b \text { ) }\end{array}$ \\
\hline
\end{tabular}

In summary, data from the literature as well as the results of the current study showed that treatment of PCa cells with the ER $\beta$ agonist $8 \beta$-VE2 induced apoptosis. However, the underlying molecular mechanisms are still unknown and it is controversial discussed which apoptosis-inducing pathway is activated and whether the effects are AR-dependent or ARindependent. The present study found an activation of AR target gene expression which seems to be contradictory to the observed effects on PCa cell survival. The whole mRNAsequencing analysis identified the most up- and downregulated genes upon 8 $\beta$-VE2 treatment. 6 out of 20 genes are known to be AR regulated. Furthermore, we found upregulation of the expression of tumor suppressive genes and downregulation of the 
expression of oncogenic genes after $8 \beta$-VE2 in VCaP cells. Further analysis of the signaling pathways underlying the $8 \beta$-VE2-induced effects in PCa cells should elucidate how these findings could be explained.

\subsubsection{Are the 8$\beta$-VE2-induced effects dependent on ER $\beta$ signaling?}

As described above, different in vitro and in vivo studies showed that ER $\beta$ exhibits tumor suppressive function in PCa (Dey et al. 2014; McPherson et al. 2010; Slusarz et al. 2012). Consistent with the current knowledge, the treatment of VCaP cell variants with the ER $\beta$ agonist $8 \beta$-VE2 induced cell survival reduction and apoptosis. The present study furthermore aims to elucidate the molecular signaling in $8 \beta$-VE2-treated PCa cells. Therefore, mRNA analysis, whole mRNA-sequencing analysis and protein analysis were performed to identify pathways involved in the signaling process. Although protein analysis showed strong expression of ER $\beta$ in VCaP cells, mRNA and whole mRNA-sequencing analysis of VCaP cell variants detected only minor ER $\beta$ expression. As described above (see 4.2), it remains unclear whether the observed ER $\beta$ protein expression results from the unspecific binding of the ER $\beta$ antibody or not. Despite this inconsistency, further analyses revealed that ER $\beta$ activation is presumably not the only mediator of $8 \beta$-VE2-induced effects in VCaP cell variants. Only one gene, namely tubulin alpha $3 d$ which is known to be regulated by ER $\beta$ in PCa was differentially expressed in 8$\beta$-VE2-treated VCaP cell variants (Vivar et al. 2010; Dey et al. 2012). Tubulin alpha $3 d$ was not described to be deregulated during to PCa development so far. However, little is known about ER $\beta$-mediated gene expression, especially in $\mathrm{PCa}$. Furthermore, the treatment of $\mathrm{VCaP}$ variants with the ER $\beta$ agonists ERB 041 and FERb 033 could just partially mimic the effect of $8 \beta$-VE2 treatment. While ERB 041 treatment effects did not concur with 8$\beta$-VE2 treatment effects in VCaP cell variants, FERb 033 reduced cell survival and induced apoptosis in VCaP cell variants but did not affect either AR gene expression or AR-regulated gene expression. FERb 033 partially mimics the effects induced by $8 \beta-V E 2$, indicating that ER $\beta$ might be involved in the induction of apoptosis. However, the ER $\beta$ antagonist fulvestrant could not inhibit 8 $\beta$-VE2-mediated effects in VCaP cells. Furthermore, the reporter assay could not show any transcriptional activity of ER $\beta$, even not for the E2-stimuated positive control. Taken together, even though the main question of the present study was to elucidate the role of ER $\beta$-mediated signaling in $\mathrm{PCa}$, the results of the present study indicate that another, to date unknown mechanism could be responsible for the $8 \beta$-VE2-induced effects in VCaP cell variants. 


\subsubsection{Are the 8 $\beta$-VE2-induced effects in VCaP cells dependent on the cholesterol synthesis pathway?}

Cholesterol is synthesized in every cell of animals, because it is an essential compound of the cell membrane. Cholesterol maintains cell membrane structure and fluidity (Cooper 1978). Furthermore, it is the precursor of steroid hormones and therefore essential for androgen synthesis (Mostaghel 2013). High cholesterol levels were associated with increased risk for different malignancies, i.e. arteriosclerosis (Ravnskov 2002). However, a cause-effect relationship is still controversial discussed (Ravnskov 2002). A connection between cholesterol and PCa was first described by Swyer et al. (1942). Swyer and coworkers observed increased cholesterol concentrations in human $\mathrm{PCa}$ compared to normal prostate tissue. More recently, it was hypothesized that the connection between intake of high fat diet and increased PCa risk might be based on the increased consumption of cholesterol (Hammarsten, Hogstedt 2002; Veierod et al. 1997). Further research showed that cholesterol synthesis is involved in PCa progression (Montgomery et al. 2008; Pelton et al. 2012). Moreover, connections between the inhibition of cholesterol synthesis, decreased proliferation, induction of apoptosis and decreased cell migration and invasion of PCa cells were described (Murtola et al. 2012; Freeman, Solomon 2004). In the present work, the whole mRNA-sequencing analysis of $8 \beta$-VE2-treated VCaP cell variants revealed that 6 out of 13 genes encoding essential enzymes of the cholesterol synthesis pathway are upregulated after $8 \beta$-VE2 treatment (see table 3.2). We hypothesized that the upregulation of the gene expression of essential enzymes of the cholesterol synthesis pathway could be induced by the loss of feedback inhibition of steroid hormones. This hypothesis is based on the assumed mechanism of action of the 5'-androstenediol HE3235 described above, which induced similar effects in LNCaP cells compared to the effects induced by $8 \beta-V E 2$ in VCaP cells. HE3235 was supposed to act as an inhibitor of the cholesterol side chain cleavage enzyme (P450scc) (Montgomery et al., 2010). P450scc mediates the conversion of cholesterol to pregnenolone, a precursor of steroid hormones such as DHT and estradiol. Therefore, P450scc is a key regulator of steroid synthesis. A hypothesized mechanism of action of $8 \beta$-VE2 including the cholesterol synthesis pathway could be that $8 \beta-V E 2$, comparable to HE3235, acts as a P450scc inhibitor and thereby blocks the production of steroid hormones. Steroid hormones and the cholesterol synthesis pathway are known to be connected via feedback inhibition (Roitelman, Simoni 1992). Blockade of steroid production by inhibition of P450scc via 8ß-VE2 could lead to the loss of feedback inhibition. And thus, this loss of inhibition could induce the expression of enzymes involved in cholesterol synthesis (Fig. 4.3). However, accumulation of cholesterol should itself induce feedback inhibition of cholesterol production (DeBose-Boyd 2008). 


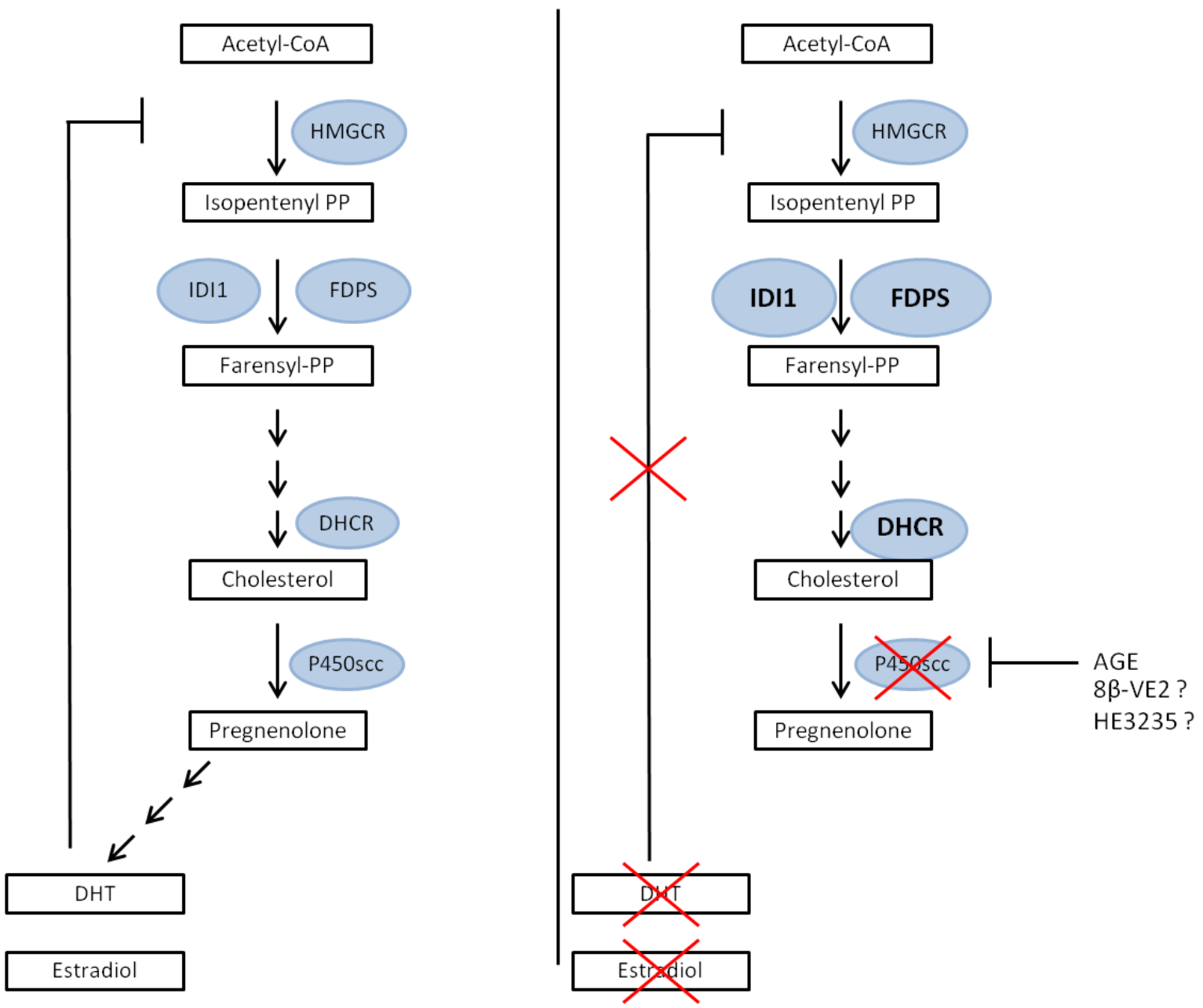

Figure 4.3: Schematic illustration of the cholesterol synthesis pathway and the putative role of

$\mathbf{8} \boldsymbol{\beta}$-VE2. Cholesterol synthesis is mediated by enzymatic reactions that convert Acetyl-CoA into cholesterol. Cholesterol is either integrated into the cell membrane or can be further metabolized to steroid hormones. The cholesterol synthesis pathway is regulated via feedback inhibition. Abundant steroid hormones can inhibit the activity of the enzyme HMGCR and therefore cholesterol synthesis. AGE (and possibly also 8ß-VE2 and HE3235) inhibits the enzyme P450scc and shuts down steroid synthesis. Due to the loss of feedback inhibition by steroid hormones the expression of enzymes of the cholesterol synthesis pathways (e.g. IDI1, FDPS and DHCR) could be increased in order to compensate the lack of steroid hormones. AGE: aminoglutethimide, DHCR: dehydrocholesterol reductase, DHT: dihydrotestosterone FDPS: farnesyl diphosphate synthase, HMGCR: 3-hydroxy-3methylglutaryl-CoA reductase, IDI1: isopentenyl-diphosphate delta isomerase 1, P450scc: cholesterol side chain cleavage enzyme. Modified according to Cyster et al. (2014), Wilcox et al. (2007), DeBoseBoyd (2008) and Roitelman, Simoni (1992).

In the present study, treatment of VCaP cells with the P450scc inhibitor aminoglutethimide (AGE) could not mimic the 8$\beta$-VE2-induced effects, i.e. P450scc inhibition could neither upregulate expression of genes involved in the cholesterol synthesis pathway nor induce ARregulated gene expression. Here, we cannot determine if $8 \beta$-VE2 inhibits P450scc, since we did not examine the activity of P450scc after 8ß-VE2 treatment in VCaP cell variants. 
Nevertheless, we can exclude that inhibition of P450scc is the underlying mechanism of action of $8 \beta$-VE2 on gene expression in VCaP cell variants.

\subsubsection{Are the 8 $\beta$-VE2-induced effects in VCaP cells dependent on PI3K signaling?}

PI3K (phosphatidylinositide 3-kinase) signaling is a cell survival and proliferation promoting pathway, which is often deregulated in cancer (Liu et al. 2009). PI3K activates its downstream target AKT which is a major regulator of a complex signal transduction network (Hers et al. 2011). In PCa, the PI3K pathway is one of the most important pathways next to AR-induced signaling (Edlind, Hsieh 2014). The inhibition of PI3K signaling in PCa treatment is currently under intense investigation, because it was shown that the inhibition of the PI3K pathway decreases PCa cell survival, invasiveness and angiogenesis (Edlind, Hsieh 2014; Fang et al. 2007; Shukla et al. 2007). With regard to the observed induction of AR-regulated gene expression after $8 \beta$-VE2 treatment, it is interesting that AR signaling and PI3K signaling are connected via reciprocal feedback inhibition (Qi et al. 2015). Hence, the inhibition of the PI3K pathway results in a loss of repression of AR signaling (Fig. 4.3). Kaarbo et al. (2010) described that the effects of $\mathrm{PI} 3 \mathrm{~K}$ inhibition overcome the accompanying effects of $\mathrm{AR}$ reactivation in $\mathrm{PCa}$ cells. Despite the cell survival promoting signaling of the $A R$, the antiproliferative effect of PI3K inhibition dominated the cell's behavior. Based on this information, inhibition of PI3K might be involved in the mechanism of action of 8 $\beta$-VE2 in VCaP cells. We suggested that $8 \beta-V E 2$ disables PI3K/AKT-mediated cell survival signals and by loss of inhibition induces AR signaling (Fig. 4.4). Therefore, PI3K signaling inhibition could combine the two apparently opposing effects of $8 \beta-\mathrm{VE} 2$ in VCaP cells: apoptosis induction despite induction of AR target gene expression. Of note, PI3K signaling can be inhibited at different sites, i.e. 8 $\beta$-VE2 could inhibit PI3K signaling indirectly, e.g. by inhibition of an upstream receptor tyrosine kinase (RTK) or inhibit PI3K directly. 

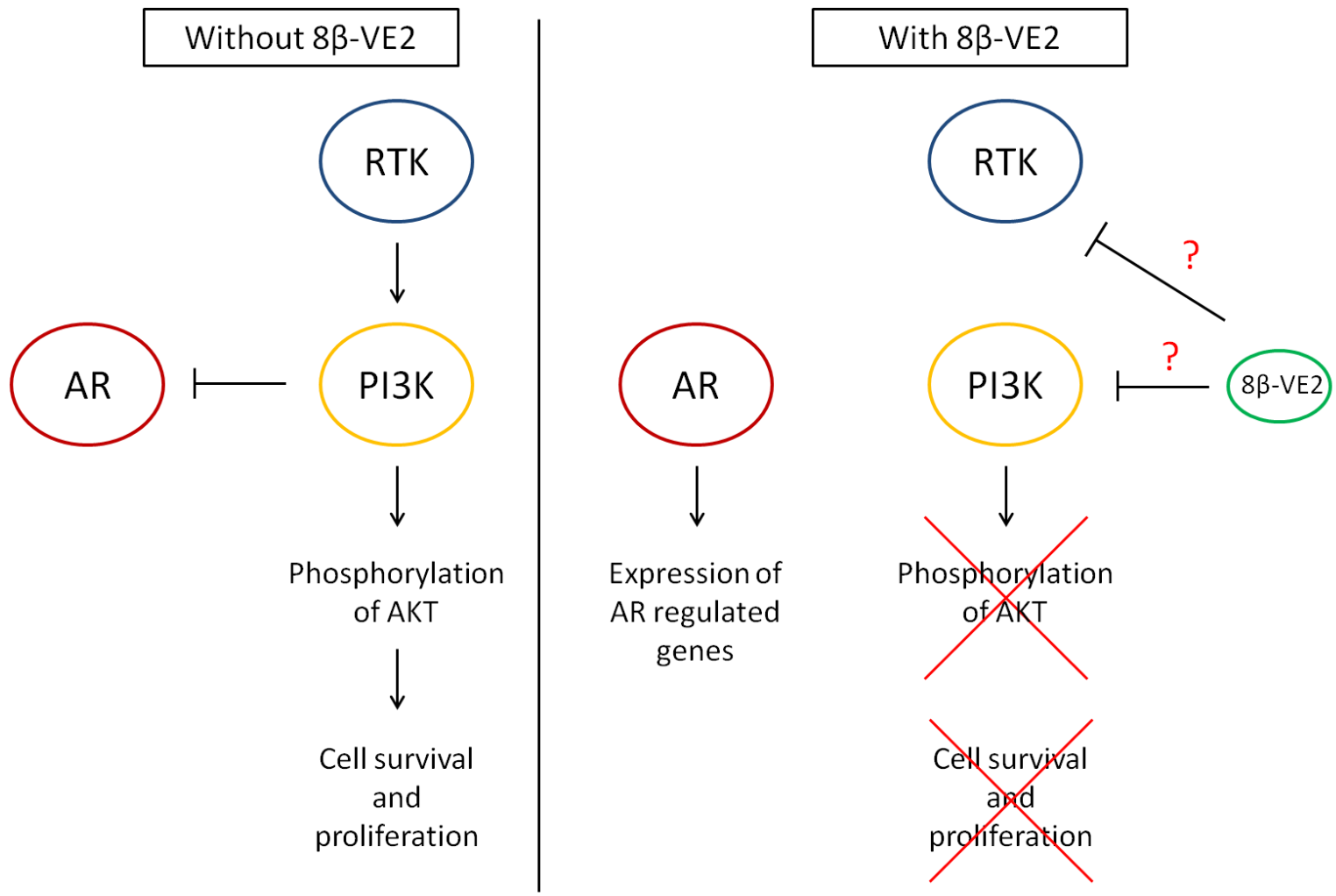

Figure 4.4: Feedback inhibition of $A R$ and PI3K signaling and potential involvement of $8 \beta$-VE2 treatment. PI3K and AR signaling are connected via feedback inhibition. Activated PI3K signaling inhibits the activation of the AR. Inhibition of PI3K signaling directly or indirectly by inhibition of upstream RTKs leads to a loss of repression of AR signaling. Therefore, PI3K inhibition leads to an inhibition of PI3K-mediated cell survival and proliferation, but also to the re-expression of AR-regulated genes. Inhibition of PI3K could explain the intrinsic contradicting effects of reduction of cell survival and an induction of AR target gene expression in VCaP cells observed after $8 \beta-\mathrm{VE} 2$ treatment. AR: androgen receptor, PI3K: phosphatidylinositide 3-kinase, RTK: receptor tyrosine kinase. Modified according to Qi et al. (2015).

However, the experiments of the current study to prove this hypothesis showed that $8 \beta-\mathrm{VE} 2$ treatment did not inhibit but induced phosphorylation of AKT and thereby caused the activation of the PI3K downstream target AKT in VCaP cells (see 3.1.2.7). Furthermore, the inhibition of PI3K signaling with the inhibitor GDC-0941 revealed that active PI3K is not necessary for mediating the $8 \beta$-VE2 treatment effects. Thus, the loss of PI3K-mediated inhibition can be excluded as an explanation for the increased expression of AR-regulated gene in VCaP cells upon $8 \beta$-VE2 treatment.

\subsubsection{Are the 8$\beta$-VE2-induced effects in VCaP cells dependent on AR signaling?}

The treatment of $\mathrm{PCa}$ cells with ER $\beta$-specific agonists should provide a new ARindependent treatment opportunity. Indeed, the observed activation of AR-regulated gene 
expression in 8 $\beta$-VE2-treated $\mathrm{VCaP}$ cell variants indicates a connection to AR signaling. However, the data collected in the present study are not fully conclusive (see Tab. 4.3).

Table 4.3: Arguments for and against AR involvement in mediation of $8 \beta$-VE2-induced effects on VCaP cell variants.

\begin{tabular}{|c|c|}
\hline AR is involved in $8 \beta$-VE2-induced effects & $\begin{array}{l}\text { AR is not involved in } 8 \beta \text {-VE2-induced } \\
\text { effects }\end{array}$ \\
\hline $\begin{array}{l}\text { - } \text { AR-negative PC3 cells did not respond to } \\
8 \beta \text {-VE2 treatment } \\
\text { - } \text { AR-regulated gene expression is induced } \\
\text { by } 8 \beta \text {-VE2 treatment }\end{array}$ & $\begin{array}{l}\text { - Only minor AR transcriptional activation } \\
\text { was observed after } 8 \beta-V E 2 \text { treatment } \\
\text { - Cell survival reduction and apoptosis } \\
\text { induction are the opposite of expected } \\
\text { effects of AR activation in PCa cells }\end{array}$ \\
\hline $\begin{array}{l}\text { - Bicalutamide activates AR transcription } \\
\text { in VCaP cells }\end{array}$ & $\begin{array}{l}\text { - DHT-induced AR activation could not } \\
\text { mimic } 8 \beta-V E 2 \text {-induced effects on cell } \\
\text { survival and apoptosis induction }\end{array}$ \\
\hline $\begin{array}{l}\text { - } \mathrm{AR} \text { and ARV expression is } \\
\text { downregulated by } 8 \beta \text {-VE2 treatment }\end{array}$ & \\
\hline
\end{tabular}

In the present study, experimental evidences were obtained after treatment of PCa cells with $8 \beta$-VE2 pointing to an involvement of the AR: 1) The treatment of AR-negative PC3 PCa cells with $8 \beta$-VE2 did not result in cell survival reduction or apoptosis induction (see 3.1.2.8.2.3). Although not experimentally validated, it is likely that the different effects of $8 \beta$ VE2 treatment in PC3 and VCaP cells are based on the most prominent difference of these two PCa cell lines which is the expression status of the AR. The AR is not expressed in PC3 cells but overexpressed in VCaP cells (Kaighn et al. 1979). This important issue indicates that the AR plays an essential role in the mediation of the $8 \beta$-VE2-induced treatment effects. 2) AR-regulated gene expression is induced by $8 \beta$-VE2 treatment. The induction of a typical AR-regulated gene expression pattern could be shown by qRT-PCR experiments and whole mRNA-sequencing analysis (see 3.1.2.2 and 3.1.2.3). The similarities of gene expression profiles between DHT-treated VCaP cells (Waltering et al. 2009) and 8 $\beta$-VE2-treated VCaP cells are highly significant $\left(p=5.5^{\star} 1033\right)$ (see 3.1.3.8). Furthermore, not only the expression of typical androgen-regulated genes matches with DHT- and 8 $\beta$-VE2-treated VCaP cells. Also randomly chosen genes to validate whole mRNA-sequencing data showed similar expression profiles after 8 $\beta$-VE2 and DHT treatment (see 3.1.2.8.2.2). A GO term pathway analysis showed that 10 of the 20 most activated pathways are similar in DHT-treated and 8ß-VE2-treated VCaP cells (compared to Cai et al. 2011) (Tab. 4.4). 
Table 4.4: Overlapping pathways activated in VCaP cells by $8 \beta$-VE2 and DHT treatment. Listed are 10 pathways activated in VCaP cells upon $8 \beta-\mathrm{VE} 2$ and DHT treatment (compared to Cai et al. (2012)).

\section{Pathways activated in 8 $\beta$-VE2-treated VCaP cells and DHT-treated VCaP cells}
1. Cholesterol biosynthetic process
2. Sterol biosynthetic process
3. Lipid biosynthetic process
4. Cholesterol metabolic process
5. Fatty acid biosynthetic process
6. Sterol metabolic process
7. Steroid biosynthetic process
8. Carboxylic acid biosynthetic process
9. Organic acid biosynthetic process
10. Unfolded protein response

Those similarities further consolidate the connection to AR-mediated signaling. 3) It was shown that the antiandrogen bicalutamide activates AR transcription in VCaP cells (see 3.1.2.8.2.1). This conversion of antiandrogenic to androgenic function indicated that it could be possible that $8 \beta$-VE2 is also accepted in VCaP cells as an AR ligand (further described below). 4) $A R$ and $A R V$ expression is downregulated by $8 \beta$-VE2 treatment (3.1.2.3). Recently, ER $\beta$ was reported to downregulate AR expression in LNCaP PCa cells (Thelen et al. 2007). But since inhibition of ER $\beta$ could not block the $8 \beta$-VE2-induced effects it seems unlikely that $A R$ downregulation is mediated by ER $\beta$ activation in the present study. Another possible explanation for the $A R$ downregulation is a self-regulating mechanism of $A R$ activation and expression driven by androgens (Quarmby et al. 1990; Shan et al. 1990). This self-regulation mechanism is mediated via $A R$ recruitment to a cis-regulatory intronic region of the AR and thus, AR itself is described to be a direct suppressor of AR gene expression (Cai et al. 2011).

Experimental evidences collected in the current study against the activation of $A R$ signaling in PCa cells upon 8 $\beta$-VE2 treatment are: 1) Only minor AR transcriptional activation was observed after $8 \beta$-VE2 treatment (see 3.1.2.8.2.4). The strongest evidence that the AR is not involved in the $8 \beta$-VE2-mediated treatment effects in PCa cells is that the reporter assay results revealed only minor induction of $A R$ transcriptional activity upon $8 \beta$-VE2 treatment. The data of this experiment demonstrated that the expression of AR-regulated target genes is not mediated by AR activation. Indeed, the activation of the AR was described to be highly dependent on AR cofactors and more than 500 AR promoter regions were identified (Bolton et al. 2007; Olsen et al. 2016). Therefore, it can be assumed that the activation of the AR by 
$8 \beta$-VE2 could specifically induced another promoter region than the one used for this assay. Another explanation would be that the expression of the typically AR-regulated genes is induced by another transcription factor (Arora et al. 2013; Fatima et al. 2015). Moreover, this assay did not give information about non-transcriptional activation of AR (Lonergan, Tindall 2011). 2) The effects of $8 \beta$-VE2 treatment on cell survival and apoptosis are the opposite effects which would be expected due to AR activation in PCa cells (see 3.1.2.2). The present study discussed about the need of restoration of $A R$ function and the adaptation mechanisms of PCa to ADT. Adaptation to ADT ensures PCa cell survival when little or no androgens are available. However, this must not be true when cellular gene expression changes. It is well documented, that patients can benefit from antiandrogen withdrawal, meaning that after a period of ADT the stop of treatment prolonged progression free survival (Paul, Breul 2000). To date, the exact mechanism how antiandrogen withdrawal affects PCa is not identified, but it is hypothesized that the relief of $A R$ signaling repression could be responsible for inhibition of PCa progression (Sartor et al. 2008; Paul, Breul 2000). Moreover, a sudden increase of androgen concentration can reduce cell survival in PCa cells, e.g. high levels of testosterone (testosterone boost) can induce proliferation inhibition and downregulation of AR and ARV expression in the castration-resistant PCa cell line VCaP (Thelen et al. 2013). In addition, beneficial effects of high dose testosterone treatment could also be shown in clinical phase I trials (Morris et al. 2009). However, both antiandrogen withdrawal syndrome- and testosterone-induced proliferation inhibition are accompanied with decreased AR-regulated gene expression, e.g. a decline of PSA expression. Taken together, AR activation could be responsible for proliferation stop in CRPC, but expression status of AR-regulated gene expression indicates that the underlying mechanism of $8 \beta$-VE2-induced effects in PCa cells differs from the antiandrogen withdrawal syndrome and from testosterone-induced proliferation inhibition. 3) Treatment of $\mathrm{VCaP}$ cell variants with low dose DHT (10 $\mathrm{nM}$ or $100 \mathrm{nM}$ ) could not mimic 8$\beta$-VE2-induced effects on PCa cell survival and apoptosis induction (see 3.1.2.8.2.2). DHT treatment of VCaP cells induced early proliferation inhibition but induction of apoptosis was not observed. Of note, DHT-induced AR activation did not induce VCaP cell proliferation. This experiment indicates that AR activation alone is not responsible for the effects observed in PCa cells upon 8 $\beta$-VE2 treatment.

In the current study experimental evidence both supporting and excluding AR signaling as the underlying mechanism of $8 \beta$-VE2-induced effects in PCa cells were found. On the one hand, the lack of AR-induced reporter gene activation is the most striking argument against the AR as the key mediator. On the other hand, the absence of treatment effects of $8 \beta-V E 2$ in AR-negative PC3 cells shows that the AR is required for induction of $8 \beta$-VE2-induced treatment effects. Hypothesizing that $A R$ activation could be the mechanism underlying the $8 \beta$-VE2-induced effects we aimed to elucidate how the AR could be activated in PCa cells. For example, the membrane bound ligand-dependent AR signaling pathway could explain 
both findings (see Fig. 4.5). First, ligand binding to the membrane-anchored AR activates second messengers like $G$ protein-coupled receptors or calcium release. Next, second messenger signaling regulates gene transcription without direct transcriptional activity of the AR. Moreover, the observed activation of PI3K upon $8 \beta$-VE2 treatment could be explained by second messenger signaling (Heinlein, Chang 2002b). Only little is known about the nongenomic signaling of the AR in PCa cells. The existence of membrane-bound AR in PCa cells was first described by Kampa et al. (2002). Most non-genomic effects are rapidly occurring within minutes after stimulation and affect e.g. calcium $\left(\mathrm{Ca}^{2+}\right)$ homeostasis and second messenger activation. Also actin cytoskeleton reorganization was observed (Papakonstanti et al. 2003). Furthermore, the activation of membrane-bound AR was shown to regulate transcriptional effects, e.g. the expression of the AR-regulated gene PSA (Kampa et al. 2002; Papakonstanti et al. 2003). However, non-genomic signaling of AR physiologically seems to mediate a rapid signal transduction but not indeed a pathway for alternative signaling. Furthermore, it could not be reasonably explained why $8 \beta$-VE2 should induce membrane-bound AR activity but not cytoplasmic AR activity.

PCa cells develop different escape mechanisms to restore AR function. AR overexpression, mutations in the AR, AR modifications (e.g. phosphorylation), constitutively active splice variants, gain and loss of cofactors and intratumoral ligand synthesis were described as escape mechanisms (Knudsen, Penning 2010) (see Fig. 1.1). VCaP cells exhibit an AR gene amplification and overexpression as an adaptation to ADT. VCaP AA cells furthermore showed the expression of constitutively active ARVs. AR overexpression was shown to be sufficient to induce PCa development (Stanbrough et al. 2001). ARVs are supposed to be important mediators of therapy resistance, since they cannot be inhibited by AR-binding site directed antiandrogens and are independent of ligand synthesis (Antonarakis et al. 2014). However, 8 $\beta$-VE2-treated VCaP and VCaP AA cells showed no further increase of $A R$ expression, in contrast, $A R$ and ARV expression was downregulated by 8 $\beta$-VE2 (see 3.1.2.3). Alternative pathways to produce testosterone or DHT are described to be active in $\mathrm{PCa}$. These pathways circumvent treatments that inhibit the AR ligand synthesis, e.g. abiraterone (Knudsen, Penning 2010). In the present study, the release of intratumoral testosterone could be excluded as the mediator of $8 \beta$-VE2-induced effects because AR target gene expression could not be abrogated in the presence of a testosterone binding antibody (see 3.1.2.8.2.1). AR posttranslational modifications are described to activate AR signaling. AR phosphorylation at different phosphorylation sites is often described to remodel AR activity (Wong et al. 2004). The induction of PI3K signaling by $8 \beta$-VE2 could point to AR activation by phosphorylation, but since we found that PI3K signaling was not necessary for $8 \beta$-VE2-mediated effects and MAPK pathway was not induced in VCaP cells, it is unlikely that ligand-independent activation of $A R$ is the underlying cause for $A R$ activation. In addition, AR target gene expression was induced after treatment with the antiandrogen 
bicalutamide (see 3.1.2.8.2.1). This result indicates that VCaP cells can accept antiandrogens as AR ligands, although VCaP cells express the wild type AR (Eskra 2015). The occurrence of mutations in the ligand binding domain, e.g. observed in LNCaP cells, could convert the function of a ligand (Veldscholte et al. 1992). Moreover, for PCa cells with wild type AR overexpression activation of AR by the estrogen E2 was described (Susa et al. 2015). Susa and coworkers induced AR overexpression in different PCa cell lines and showed nuclear AR localization and AR-specific reporter activation upon treatment with E2. Here, the authors speculated that wild type AR can be activated by E2 in certain hormonesensitive cancer cells. Similarly, Chen et al. (2004) described that the massive overexpression of the $\mathrm{AR}$ in $\mathrm{VCaP}$ cells contributes to more unspecific ligand binding, however, the exact mechanism is still elusive. The high concentration of $8 \beta$-VE2 used in the present study combined with the massive overexpression of AR in VCaP cells could facilitate the activation of AR by $8 \beta$-VE2. The recruitment of cofactors could also contribute to the $A R$ activation. Cai et al. (2011) described that the effects of AR activation are mainly dependent on the involved coactivators and corepressors. AR coregulators are cis-regulatory elements. Several hundred coregulators of the AR are described to be involved in different processes in PCa (Heinlein, Chang 2002a). Next to regulation of transcription, coactivators are also known to regulate activity and recruitment of chromatin remodeling enzymes (e.g. histone acetylases) and induce changes of AR conformation. AR corepressors mediate the feedback inhibitory regulation of $A R$ activation to other signaling pathways, i.e. cell cycle regulation (Knudsen, Penning 2010). Both, gain of coactivators and loss of corepressors are described to be involved in antagonist to agonist conversion (Burd et al. 2006; Wang et al. 2009).

The agent HE3235 was described to be a synthetic AED (Trauger et al. 2009). AEDs are potent ER- as well as AR activators and possibly act as a ER $\beta$-AR transactivator (Maggiolini et al. 2004). Interestingly, AED-mediated AR activation cannot be blocked by the antiandrogens flutamide or bicalutamide (Miyamoto et al. 1998). Due to the comparable effects of HE3235 and $8 \beta$-VE2 it would be reasonable to suggest a similar signaling mechanism for $8 \beta$-VE2. Nevertheless, in contrast to HE3235 and 8 $\beta$-VE2, ER $\beta$-AR transactivation was reported to promote cell survival and proliferation in the PCa cell line LNCaP (Maggiolini et al. 2004). Identification and analysis of potential involved cofactors was not performed in the present study and could be an interesting field for further analysis.

Taken together, AR signaling is presumably involved in the mediation of $8 \beta$-VE2-induced effects. The similarities of gene expression between DHT- and 8 $\beta$-VE2-treated VCaP cells and the lack of effects in AR-negative PCa cells strongly indicate the central role of the AR, but the lack of strong AR transcriptional activation in 8 $\beta$-VE2-treated VCaP cells exclude direct transcriptional regulation. Possible explanations could be the interaction of the AR with different coactivators and corepressors, activation of membrane-bound AR signaling, 
transactivation of $E R \beta$ and $A R$ or activation of an unknown transcription factor mimicking the AR (Fig 4.5).

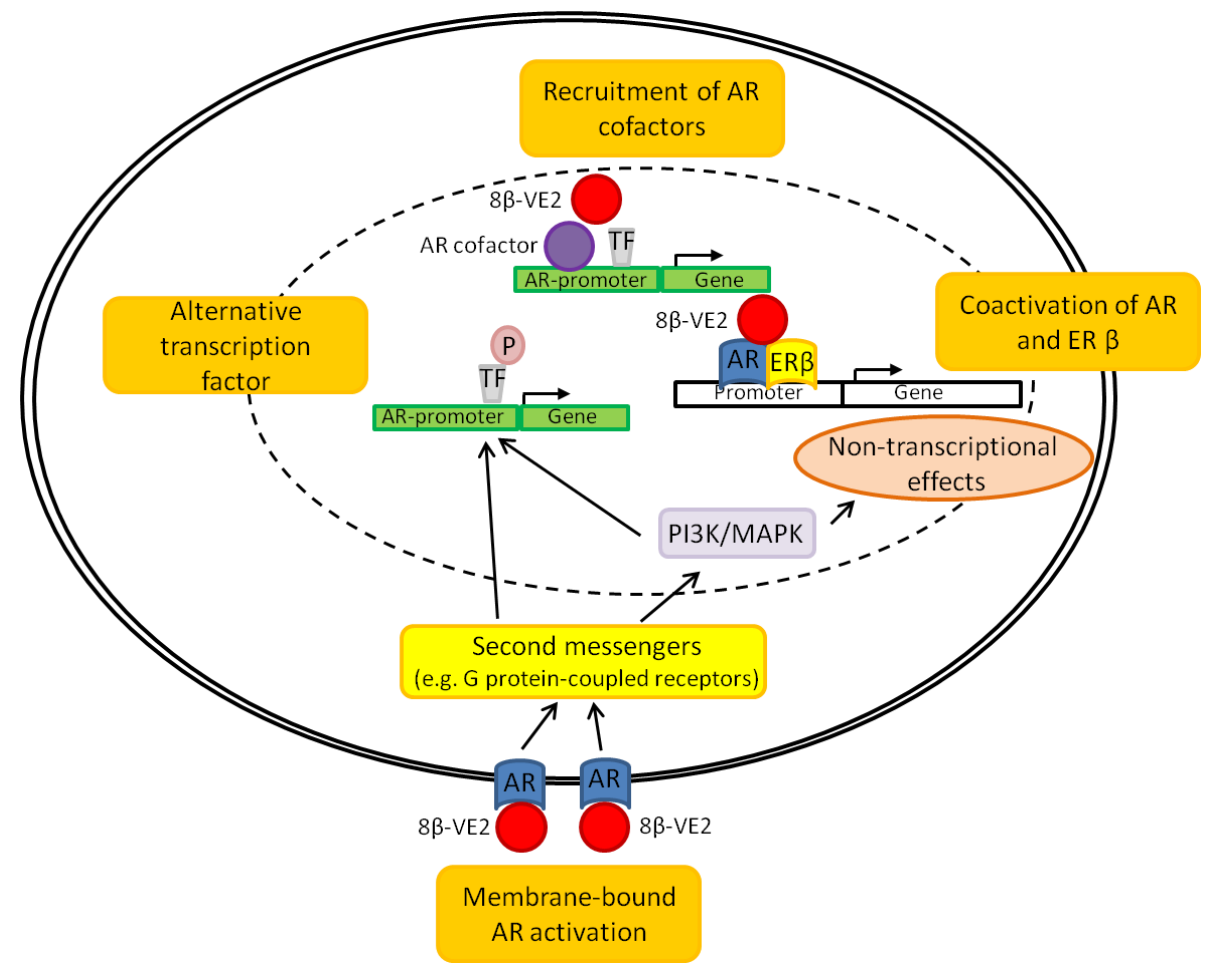

Figure 4.5: Possible mechanisms of AR activation in $8 \beta$-VE2-treated VCaP cells. AR signaling could mediate the $8 \beta$-VE2-induced effects by membrane-bound AR signaling transduction and activation of second messengers such as $G$ protein-coupled receptors. AR target gene expression could be induced by activation of alternative transcription factors, coactivation of $E R \beta$ and AR or via the recruitment of AR cofactors. Modified according to Lonergan, Tindall (2011) and Maggiolini et al. (2004)

\subsection{AR-mediated alternative survival pathways}

AR signaling is not only important in prostate cancer, but also in healthy prostate tissue (Singh et al. 2014). In PCa tissue AR signaling is the most important cell survival signaling (Lonergan, Tindall 2011). However, the PCa development cannot be sufficiently explained by increased activation of $A R$ signaling, since the $A R$ exhibits activating and repressive functions (Karantanos et al. 2013). In healthy prostate tissue and early PCa stages AR signaling is a regulator of both repressive and activating pathways. Cai et al. (2011) described activation of pathways involved in lipid and protein synthesis and repression of pathways regulating DNA synthesis and the cell cycle. When the balance between repressive and activating functions of the $A R$ is lost, aggressive $\mathrm{PCa}$ cells evolve (Holzbeierlein et al. 2004; Cai et al. 2011). This means, that not a fully restored function of the $A R$ is achieved in advanced PCa, but a partially active AR with activating function, but with a loss of the repressive signaling machinery (Cai et al. 2011). 
In the previous chapter the auto-regulation of the AR together with a proliferation inhibition after testosterone treatment is described (Cai et al. 2011; Sartor et al. 2008; Paul, Breul 2000). Similar effects can be observed in VCaP rev. cells and in 8 $\beta$-VE2-treated VCaP cells and VCaP AA cells. When first treated with testosterone or $8 \beta-\mathrm{VE} 2$, cell proliferation of VCaP cells was inhibited (Thelen et al. 2013). Long term testosterone treatment of VCaP cells results in the generation of the VCaP rev. cell line which can be differentiated from VCaP cells by its gene expression profile (see 3.1.2.1 and 3.1.2.3). VCaP rev. cells and 8 $\beta$-VE2treated VCaP and VCaP AA cells exhibit a remarkably lower expression of AR, supporting the evidence for AR auto-regulation. Furthermore, the data indicate that adaptation to ADT (when it is achieved by AR overexpression) can be at least partially restored. Downregulation of AR- and ARV expression in VCaP rev. cells as well as 8 $\beta$-VE2-treated VCaP and VCaP $A A$ cells on the one hand, downregulation of proliferation and induction of apoptosis in $8 \beta$ VE2-treated VCaP and VCaP AA cells on the other hand point to a reversion of the PCa cell phenotype back towards a less aggressive pre-ADT PCa cell type. The results obtained from the whole mRNA-sequencing analysis of the different VCaP cell variants support this hypothesis. The highest consensus of gene expression was found in VCaP rev. and VCaP and VCaP AA cells treated with $8 \beta$-VE2. The equalization of the expression profiles point to a more similar phenotype. Cai et al. (2011) and Karantanos et al. (2013) suggested an androgen-dependent regulation of $\mathrm{AR}$ expression and regulation. This model involves two distinct AR-regulated pathways. On the one hand, the regulation of the expression of common AR target genes, on the other hand the regulation of the alternative survival pathway. Based on the data generated in this study, we hypothesize that the VCaP cell variants resemble the different PCa cell stages described by Cai et al. (2011). With increasing adaptation to ADT repression of the alternative survival pathway decreases and thus survival of PCa cells is ensured. However, the $8 \beta$-VE2-mediated induction of apoptosis might be due to the shutdown of the alternative survival pathway and the fully restored $A R$ function back to pre-ADT state (Fig. 4.6). 


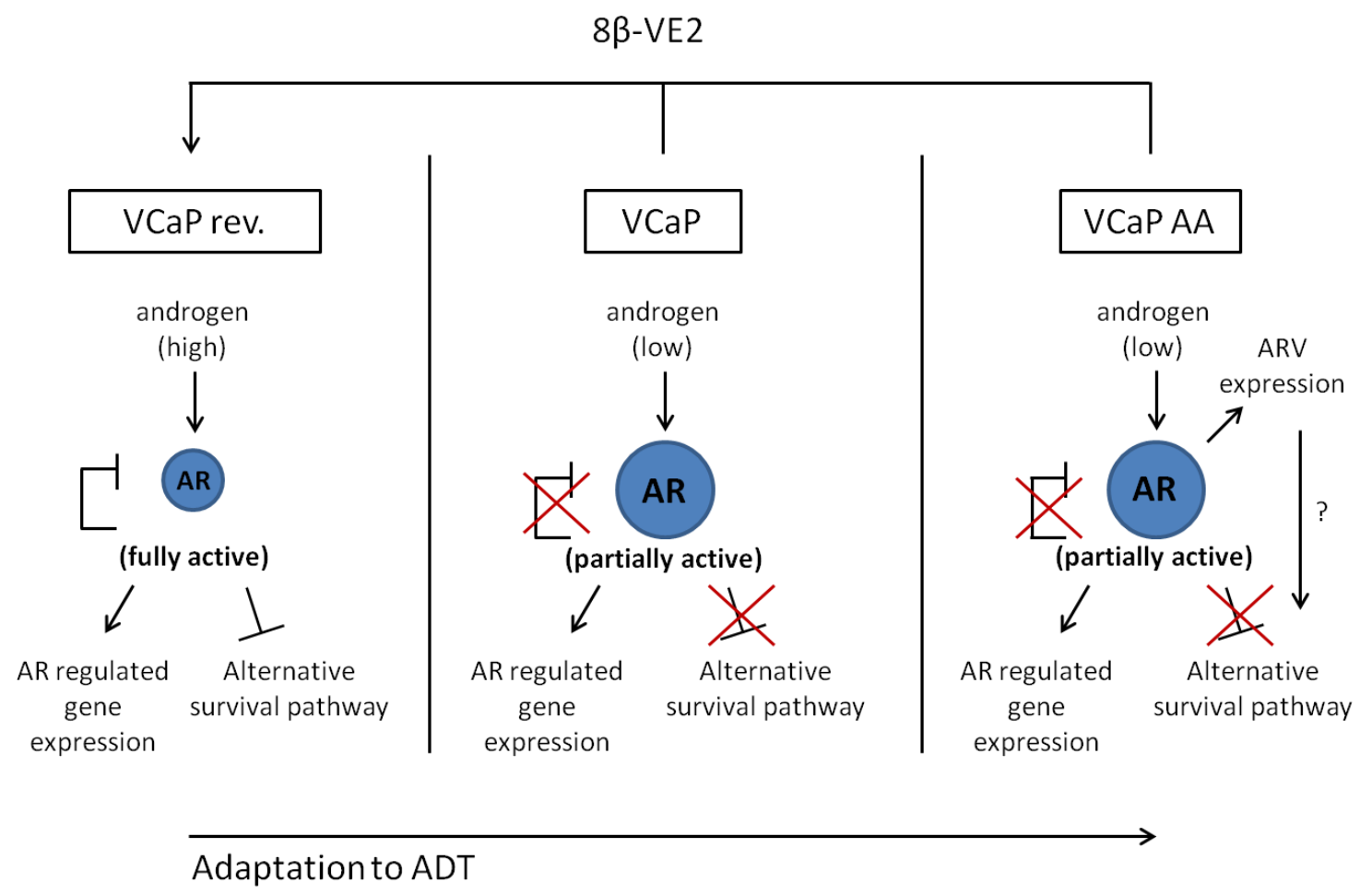

Figure 4.6: AR-regulation in PCa changes during disease progression. In early PCa (VCaP rev.) high androgen levels led to full active AR. AR signaling auto-regulates AR expression and regulation of AR-induced and -repressed pathways is relative intact. PCa cells adapt to ADT downregulated androgen level. VCaP cells exhibited partial restored AR function. Here, AR repressed signaling (AR auto-regulation and alternative survival pathway) is lost. In VCaP AA cells the signaling is even more deregulated by expression of ARVs. We assume that $8 \beta-V E 2$ treatment restored fully active $A R$ function and thus shut down the alternative survival pathway. Modified according to Cai et al. (2011) and Karantanos et al. (2013).

The AR-mediated alternative survival pathway is not identified so far. Candidates need to be differentially regulated in non-tumoral prostate or early PCa and advanced PCa. Karantanos et al. (2013) suggested different pathways to be potentially involved in the AR-regulated alternative survival pathway: 1) Overexpression of the proto-oncogene c-myc. C-myc overexpression was frequently observed in CRPC and ADT has been suggested to increase the incidence of c-myc overexpression (Nupponen et al. 1998; Visakorpi et al. 1995). Moreover, advanced PCa cells were found to grow under ADT when c-myc is overexpressed (Bernard et al. 2003). However, whole mRNA-sequencing analysis could not detect differential expression of c-myc, therefore it is unlikely that c-myc is involved in the effects described in the present study. 2) Activation of PI3K or MAPK signaling. As described above, PI3K signaling is an important survival promoting pathway in $\mathrm{PCa}$, but could be excluded to be the regulator for the $8 \beta$-VE2-mediated treatment effects. No activation of MAPK signaling was found in untreated and $8 \beta$-VE2-treated VCaP cells, therefore also MAPK signaling can be excluded as the mediator of the AR-regulated alternative survival pathway. 3) Activation 
of the $W n t / \beta$-catenin pathway. The $W n t / \beta$-catenin pathway is an important regulatory pathway during embryogenesis and was shown to be deregulated and associated with cancer formation in different tissues, among others also in the prostate. Wnt/ $\beta$-catenin is a cell survival promoting signaling pathway (Kypta, Waxman 2012). The connection between $A R$ signaling and Wnt/B-catenin is not fully understood, but it is suggested that $A R$ signaling inhibits Wnt/ $\beta$-catenin signaling (Chesire, Isaacs 2002; Wan et al. 2012). Interestingly, Yokoyama et al. (2014) described changes in the signaling regulation during the malignant degeneration of PCa. In primary PCa AR signaling inhibits Wnt/ $\beta$-catenin signaling whereas in CRPC AR and Wnt/B-catenin induce each other. In the present study, whole mRNAsequencing analysis and qRT-PCR experiments showed downregulation of Wnt2 expression upon $8 \beta$-VE2 treatment in VCaP and VCaP AA cells. Nonetheless, no differential expression of $\beta$-catenin could be detected. $\beta$-catenin signaling is mainly regulated by protein stabilization and degradation and therefore might not be detected by mRNA analysis. Thus, Wnt/ $\beta$ catenin signaling might play a role in 8 8 -VE2-mediated treatment effects (Fig. 4.7). 4) Epidermal growth factor (EGF)/ epidermal growth factor receptor (EGFR) pathway is another interesting candidate which was not suggested by Karantanos et al. (2013) but identified based on an in silico upstream analysis of the whole mRNA-sequencing data generated in the current study. Due to the in silico analysis the downregulation of EGFmediated signaling was identified to be the most likely regulator for differential gene expression between untreated- and 8ß-VE2-treated VCaP and VCaP AA cells. EGF/EGFR signaling plays an important role in prostate morphogenesis and was furthermore connected to prostate tumorigenesis (Kim et al. 1999). The connection between EGF/EGFR and AR signaling was described in different publications. Leotoing et al. (2007) hypothesized that in normal prostate tissue an inverse correlation between EGF and AR signaling exists, indicating underlying feedback inhibition. During prostate tumor progression overexpression of EGF and EGFR were described to promote cancer cell proliferation and invasiveness (Jarrard et al. 1994), whereas EGFR inhibition induces apoptosis in PCa cells (Festuccia et al. 2005). Moreover, EGFR was reported to be connected to the cholesterol synthesis pathway in PCa (Freeman et al. 2007). As described above, a deregulation of cholesterol synthesis was induced upon $8 \beta-V E 2$ treatment. Therefore, the EGF/EGFR pathway connects a number of effects observed after $8 \beta$-VE2 treatment of VCaP cell variants and thus is also a potential candidate for the AR-mediated alternative survival pathway (Fig. 4.7). 


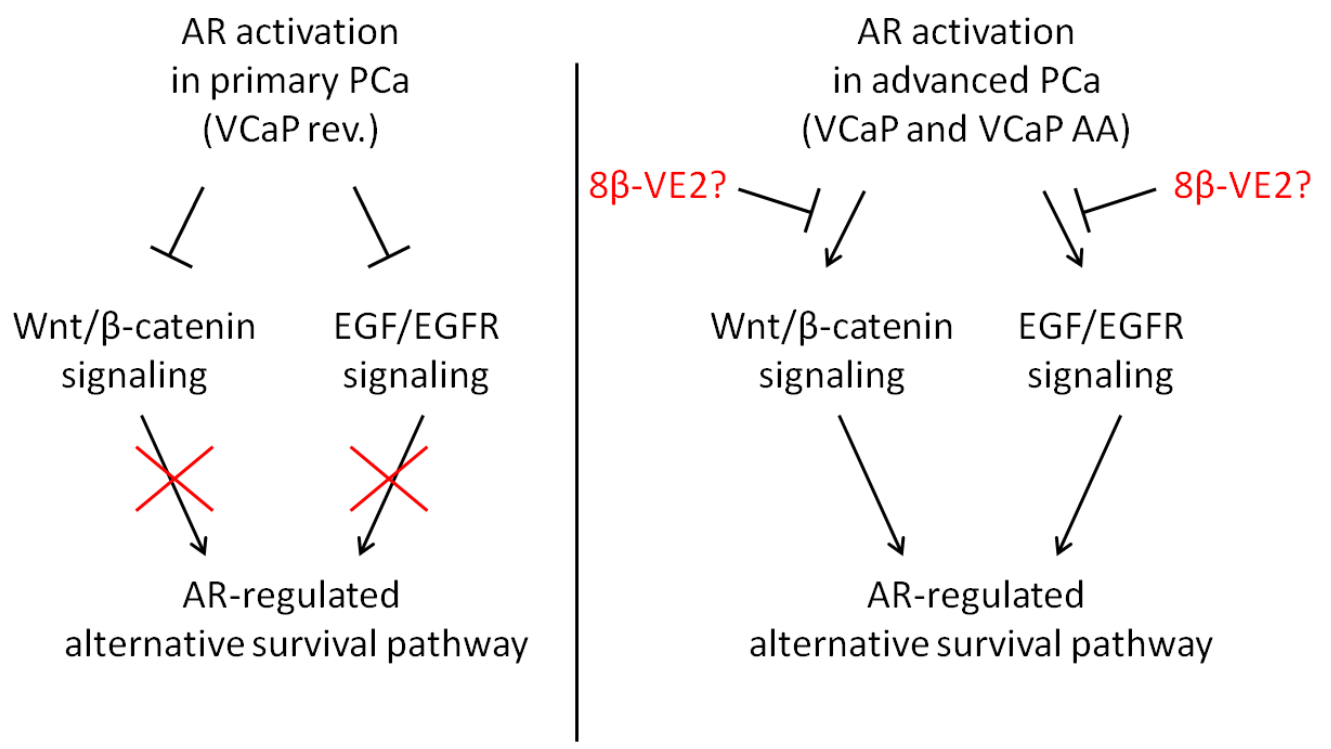

Figure 4.7: Potential role of $\mathrm{Wnt} / \boldsymbol{\beta}$ catenin signaling and EGF/EGFR signaling in PCa cell survival. The activation of an alternative AR-regulated survival pathway could be a possible explanation for the development of PCa resistance to ADT. Moreover, inhibition of the alternative survival pathway could be the underlying mechanism for $8 \beta$-VE2-mediated treatment effects in PCa cells. With respect to the literature, we suggest two possible candidates for mediating the ARregulated alternative survival pathways based on the whole mRNA-sequencing analyses: Wnt/ $\beta$ catenin signaling and EGF/EGFR signaling. Both pathways were described to be inhibited in primary $\mathrm{PCa}$ (VCaP rev. cells) and induced during PCa progression (VCaP and VCaP AA cells). We hypothesize that $8 \beta$-VE2 inhibits the signaling of $W n t / \beta$-catenin or EGF/EGFR signaling axis.

Taken together, both $\mathrm{Wnt} / \beta$-catenin signaling and the EGF/EGFR pathway are putative candidates for the AR-regulated alternative survival pathway.

\subsection{Amygdalin- a potent anti-cancer agent or toxic nonsense?}

The physiological effects of amygdalin are controversially discussed. Whereas proponents of amygdalin assert that amygdalin exhibits potent anti-cancer potential, opponents claim amygdalin to be toxic, dangerous and only applied by dubious profit-orientated quacks. In Germany, the use of amygdalin is prohibited and the DKFZ (Deutsches Krebsforschungszentrum) advise patients against the risk of cyanide intoxication after the use of amygdalin. However, different current in vitro studies underpin anti-cancerous features of amygdalin in PCa cells, but reliable in vivo studies are missing (Chang et al. 2005; Chang et al. 2006; Makarevic et al. 2014; Makarevic et al. 2016). Furthermore, it remains unclear how amygdalin may affect cancer cells.

Partially responsible for the controversial results concerning amygdalins efficacy is confusion in the nomenclature. Often the terms laetrile, vitamin B17 and amygdalin are used 
synonymously though indeed describe different more or less purified substances. Amygdalin is the natural occurring cyanogenic diglucoside, composed of two glucose molecules, one benzaldehyde group and one cyanide ion. In contrast, laetrile, also called vitamin B17, is a semi-synthetic cyanogenic glucoside composed of one glucose molecule, one benzaldehyde group and one cyanide ion (Unproven methods of cancer management. Laetrile 1991). Even more confusing, although called vitamin B17, laetrile is not a vitamin, since it is not required for any physiological processes. Amygdalin was termed to be too toxic for human use by Dr. Krebs Sr. and subsequently his son Dr. Krebs Jr. produced the supposedly less toxic laetrile. Indeed, the synthesis of laetrile did not work efficiently and mostly amygdalin was produced instead of laetrile (Unproven methods of cancer management. Laetrile 1991). Many studies, i.e. Moertel et al. (1981) and Milazzo et al. (2011) did not clearly indicate if amygdalin or laetrile was used, but use the terms synonymously. However, in the present study amygdalin was used for both in vivo and in vitro experiments.

\subsubsection{Toxicity and mechanism of metabolisation}

Next to its debatable efficacy, most concerns about amygdalin consider its toxic side effects. Cases were reported where patients showed signs of intoxication after consume of high amounts of amygdalin (Milazzo et al. 2011). Especially the oral intake of amygdalin was shown to be associated with increased risk for toxic side effects due to increased bloodcyanide concentrations in humans (Moertel et al. 1981). Indeed, blood-cyanide concentrations varied strongly between patients although similar amygdalin amounts were consumed (Moertel et al. 1981). Therefore, safe amounts of amygdalin are difficult to define, but in humans $0.5-3.5 \mathrm{mg} / \mathrm{kg}$ cyanide are lethal (equals a maximal intake of $62.5 \mathrm{mg} / \mathrm{kg}$ amygdalin) (Bundesinstitut für Risikobewertung, 2014). For mice, rabbits and dogs the maximal tolerated dose is $3 \mathrm{~g} / \mathrm{kg}$ administered intravenously and $0.075 \mathrm{~g} / \mathrm{kg}$ administered orally (Rauws et al. 1982; Zhang, Jin 1986; Khandekar, Edelman 1979). However, consumption of smaller amounts of amygdalin seems to be harmless (Carter et al. 1980). Intravenous amygdalin injections did not increase blood-cyanide concentrations and were not reported to cause side effects (Moertel et al. 1981). In the present mouse study, amygdalin was applied by i.p. injections. Neither toxic side effects, such as weight loss or organ health impairment, nor increased blood-cyanide concentrations were found.

Although numerous studies about amygdalin were performed, it is still unsolved how amygdalin may affect cancer cells. The most common hypothesis described the enzymatic cleavage of amygdalin by $\beta$-glucosidase into cyanide, benzaldehyde and glucose (Haisman, Knight 1967). Of note, laetrile cannot be cleaved by this enzymatic reaction (Unproven methods of cancer management. Laetrile 1991). Also the expression of the enzyme rhodanase is supposed to be important. High rhodanase expression should lead to fast detoxication of cyanide and therefore counteracts the anti-cancerous effects. According to 
the hypothesized mechanism of action, tumor cells exhibit higher expression level of $\beta$ glucosidase and lower rhodanase expression than normal tissues. Therefore, more amygdalin is cleaved and more cyanide released. This phenomenon should determine tumor cell specificity of amygdalin. However, Carter et al (1980) reported that $\beta$-glucosidase is not expressed in human tumor cells but in gut epithelial cells. To investigate the effects of amygdalin in vivo it is important to know, how amygdalin should be applied. The choice of application routes decide if amygdalin is metabolized during the process of drug intake. In case of oral application the passage of gut and liver is needed before the drug arrive the blood circulatory system. The expression of $\beta$-glucosidase in gut epithelial cells leads to a first-pass effect, i.e. that the drug is metabolized before it reaches its actual point of action. This effect explains why oral amygdalin intake is associated with increased blood-cyanide concentrations, whereas injected amygdalin circumvents the gut passage and thus does not release cyanide systemically. The question remains, if amygdalin can only be metabolized in the human gut and therefore the first-pass effect is needed to release the active compound of amygdalin or if cyanide release in the gut is an undesired effect and prevents amygdalins effectiveness in tumor cells. It is also possible, that amygdalin does not need to be cleaved to be effective on cancer cells and cleavage of amygdalin in the gut therefore prevents amygdalins' positive effects. In the present study it was shown that mouse PCa tumor tissue derived from TRAMP-FVB mice expressed $\beta$-glucosidase and rhodanase. Therefore, the parenteral application should be able to induce effects of amygdalin. Considering the $\beta$ glucosidase/rhodanase expression ratio, cyanide should be released into the tumor tissue, but even to a higher amount in the spleen (see 3.2.1.4.1). However, signs of toxic degeneration were not found in the spleen or in any other tissues analyzed. Only in tumor tissue an increased formation of necrotic tissue in amygdalin-treated compared to untreated mice was observed. These data could not fully elucidate the mechanism of action of amygdalin. The observation of increased necrosis in prostate tumors without cellular degeneration in spleen and other tissues, together with the lack of cyanide excretion after parenteral administration indicates a tumor-specific effect of amygdalin. However, this effect could not be explained by the expression pattern of $\beta$-glucosidase and rhodanase. Indeed, Moon et al. (2015) reported that amygdalin metabolism is achieved by an increased activation of $\beta$-glucosidase rather than increased $\beta$-glucosidase expression level. Since activation of $\beta$-glucosidase was not analyzed in the present study, no conclusions about the activity of $\beta$-glucosidase in prostate tumor and spleen could be drawn.

In summary, we demonstrated that parenteral administration of amygdalin did not induce any toxic side effects in TRAMP mice. Furthermore, the enzymes $\beta$-glucosidase and rhodanase are expressed in different tissues, such as kidney, spleen, liver, lung and PCa tissue. However, the expression of these enzymes cannot explain the prostate tumor specificity of amygdalin-induced effects. 


\subsubsection{Amygdalin reduces the invasive potential of $\mathrm{PCa}$}

The invasive potential of cancer cells is one of the major hallmarks of tumor progression (Hanahan, Weinberg 2011). In most cases not the primary tumors but metastases are responsible for the fatal outcome of cancer disease. In patients with $\mathrm{PCa}$ metastasis formation occurs mostly in bone (90\%), lung (46\%) and liver (25\%) (Bubendorf et al. 2000). To invade other tissues, cancer cells need to escape the primary tumor site, invade lymphatic or blood vessels and grow on distant organs to form a metastatic site. Different modifications in gene expression are necessary for this complex process and the mechanism of invasion is still not fully understood. Changes in the expression profile of cancer cells, epithelial-mesenchymal transition (EMT) and changes in tumor microenvironment were described to be associated with tumor cell invasion (Hanahan, Weinberg 2011). In the present study, we could demonstrate that amygdalin reduces the invasive potential of PCa cells in vitro and reduces metastasis formation in preventive-treated TRAMP-FVB mice. Antiinvasive potential of amygdalin was described for different cancer cell lines in vitro, e.g. renal cell carcinoma, bladder cancer and non-small cell lung cancer (Juengel et al. 2016; Makarevic et al. 2014; Qian et al. 2015). Juengel et al. (2016) and Makarevic et al. (2014) described that the anti-invasive effect of amygdalin is mediated by an integrin-dependent mechanism, impairing cell adhesion and invasion. Integrins are transmembrane glycoprotein receptors. They are major regulators of cell adhesion and involved in gene expression, affecting the regulation of cell survival, differentiation and proliferation (Koistinen, Heino 2013). Integrins are described to be involved in the initiation and progression of cancer cell invasion (Guo, Giancotti 2004). Differential expression of integrins was also observed in PCa and found to be correlated to PCa clinical outcome (Hess et al. 2014). Makarevic et al. (2014) hypothesized that amygdalins' anti-invasive effect is mediated via downregulation of $\beta 1$ and $\beta 4$ integrin expression. However, how amygdalin affects gene expression was not described.

Taken together, the present study hypothesizes that amygdalin has anti-invasive effects on PCa cells. Whether or not downregulation of integrin expression is involved in this effect is under investigation.

\subsubsection{Amygdalin treatment induces senescence in vitro}

We could show that the agent amygdalin induces senescence in the murine primary prostate cancer cell lines T244 and 2E. Upon amygdalin treatment, the cells cease proliferation without induction of apoptosis or cytotoxic cell death. Together with the time- and dosedependent changes of cell morphology and the induction of SA $\beta$-gal expression clear evidence of senescence induction was found. 
Cellular senescence is an irreversible arrest of proliferation. Senescent cells stay metabolic active and maintain cellular signaling, especially release of immunogenic cytokines (Campisi 2013). Senescent cells show morphological changes (Bayreuther et al. 1988; Cho et al. 2004) and SA $\beta$-gal expression (Dimri et al. 1995). SA $\beta$-gal staining is based on the increased expression of lysosomal $\beta$-galactosidase in senescent cells and is used as a senescence marker (Lee et al. 2006). Different causes for induction of senescence are known. Replicative senescence, also known as Hayflick limit, occurs after a cell line-specific number of cell cycles due to telomere shortening (Hayflick, Moorhead 1961). Telomeres, the DNA-protein structures that cap the ends of linear chromosomes, shorten with every cell division (Allsopp et al. 1995). Telomere shortening is associated with chromosomal instability (Murnane 2012). To prevent instability and the associated risk of cancer development p53mediated senescence is induced (Rodier et al. 2005). Accelerated senescence is induced by other factors, e.g. DNA damage, induction of DSB or mitotic catastrophe (Nakamura et al. 2008; Robles, Adami 1998). Oxidative stress or strong mitogenic activation driven by oncogenes can also induce accelerated senescence (Robles, Adami 1998; Nakamura et al. 2008; Barascu et al. 2012; Parrinello et al. 2003; Blagosklonny 2003). Furthermore, the activation of tumor suppressor genes such as p53, p21 or pRB (retinoblastoma protein) can induce and mediate senescence (Adams 2009; Roninson 2003). The secretion of immunogenic cytokines by senescent cells induces immune recognition and clearance and thereby prevents malignant degeneration (Freund et al. 2010). During their degeneration cancer cells shut down pathways that are responsible for proliferation inhibition and cell death induction. Therefore, the induction of replicative senescence as well as accelerated senescence is often circumvented in cancer cells (Dimri 2005). Moreover, it is hypothesized that the physiological function of accelerated senescence is the blockade of oncogenic degeneration. It could be shown that inhibition of senescence induction facilitates malignant degeneration of cells (Boehm, Hahn 2005).

Therefore, senescence induction is desired in cancer treatment and prevention (Collado, Serrano 2010; Prieur, Peeper 2008). Stop of proliferation of cancer cells by induction of cellular senescence was shown to be very effective in cancer treatment and prevention (Chen et al. 2005; Dimri 2005). Chen et al. (2005) reported that the induction of senescence can inhibit malignant degeneration of prostate in PTEN-deficient mice. In prostate cancer, senescence was identified, next to apoptosis and mitotic catastrophe, to be induced by classical anti-cancer treatment e.g. docetaxel (Schwarze et al. 2005). Also ADT was reported to induce senescence in PCa cell lines (Burton et al. 2013). Moon et al. (2015) reported that amygdalin treatment decreases telomerase activity in cancer cells but not in fibroblast cells. Telomerase is the enzyme is responsible for the maintenance of telomeres and therefore of essential importance for the control of replication (Greider, Blackburn 1985). Inactivation of telomerase leads to chromosomal instability and therefore potentially induces senescence 
(Xu et al. 2015). Moreover, Moon et al. (2015) demonstrate a cancer-specific effect of amygdalin treatment, the mechanism of this specificity could not be identified so far. The present study showed that DSBs and mitotic catastrophe occurs after amygdalin treatment and thus indicates that DNA damage plays a major role in the amygdalin-mediated induction of senescence. DSB as well as mitotic catastrophe can be induced by different causes and through different mechanisms. DSB can be caused by e.g. ionizing radiation, DNA intercalating chemotherapeutics (e.g. cisplatin or melphalan), potassium cyanide or the detergent Triton-X 100 (Mladenov, Iliakis 2011; Vock et al. 1998; Winn et al. 2003; Mladenov, Iliakis 2011). Amygdalin is a cyanide-containing compound, therefore it is likely that the induction of DSBs is mediated by the cyanide ion. Cyanide-containing compounds were described to induce DSBs by inhibition of the mitochondrial enzyme cytochrome oxidase (Pettersen, Cohen 1993). The inhibition of cytochrome oxidase induced a number of events, next to others, the release of reactive oxygen species (ROS) (Way 1984). ROS can bind to DNA and thereby induce DSB (Winn et al. 2003). Furthermore, ROS can directly activate p53 and therefore induces senescence (Singh et al. 2010). The exact mechanism how DSB induce senescence is still under debate. A complex network of DNA damage sensors, apical local kinases, DNA damage mediators, downstream diffusible kinases and other effectors is active and responsible for induction of either transient cell cycle arrest, senescence or apoptosis (Di d'Adda Fagagna 2008).

Mitotic catastrophe results from chromosomal instability (Vitale et al. 2011). Missegregation of chromosomes during mitosis leads to aneuploid daughter cells. Aneuploidy is not unusual in cancer cells, thus in mitotic catastrophe the chromosome integrity is damaged beyond repair. Cell division is terminated and therefore mitotic catastrophe results in either apoptosis or senescence induction (Mansilla et al. 2006; Eom et al. 2005). DNA damage can also result in mitotic catastrophe when cell cycle checkpoints are deregulated (Huang et al. 2005). Here, it is unclear if amygdalin treatment directly induces mitotic catastrophe or if mitotic catastrophe is a consequence of amygdalin-induced DSBs. The inactivation of telomerase as described by Moon et al. (2015) could also result in mitotic catastrophe (Hornsby 2007).

As described above, DSBs as well as mitotic catastrophe can induce either apoptosis or senescence. In the present study senescence was induced. Other studies report cell cycle arrests (Makarevic et al. 2016) or apoptosis induction (Chang et al. 2005) in PCa cells upon amygdalin treatment. Both, cell cycle arrest and apoptosis induction could also be consequences of DNA damage. The severity of damage as well as the molecular profile of cells seems to be responsible for which cell fate is induced, either apoptosis or cell cycle arrest (Di d'Adda Fagagna 2008; Vakifahmetoglu et al. 2008). 


\subsubsection{Amygdalin treatment induces necrosis in vivo}

In the current study TRAMP-FVB mice were treated with $100 \mathrm{mg} / \mathrm{ml}$ amygdalin and showed increased induction of necrosis in prostate tumors. It was long suggested that necrosis, in contrast to apoptosis, is an unprogrammed form of cellular death, mainly induced by injury. Necrosis is hallmarked by cell rounding, organelle swelling and membrane rupture, but no internucleosomal DNA fragmentation (Vanlangenakker et al. 2012). In contrast to apoptotic cells, necrotic cells are not cleared of by the immune system and they are associated with the release of inflammatory cytokines (Proskuryakov et al. 2003). Recent publications demonstrate that necrosis is more than chaotic cell lyses. Organized necrosis is termed necroptosis (Su et al. 2016). Different necroptotic pathways are described (tumor necrosis factor receptor 1 (TNFR1)-mediated, receptor-interacting protein (RIP1)-mediated, poly ADPribose polymerase (PARP)-mediated), indicating that necroptosis is a well-organized mechanism of cell death (Festjens et al. 2006). It was shown that identical stimuli can induce both, apoptosis and necro(pto)sis on the same cell population (Yen, Klionsky 2008). Two factors seem to be crucial for the decision whether apoptosis or necro(pto)sis is induced: severity of damage and depletion of ATP. If damage is too severe cells undergo necro(pto)sis. Apoptosis is an ATP-dependent process, therefore necro(pto)sis is induced in case of ATP depletion (Yen, Klionsky 2008). Furthermore, it was shown that apoptosis and necro(pto)sis are connected via TNFR1 and AKT. Factors like RIP1 can induce both, apoptosis and necro(pto)sis. However, induction of necro(pto)sis inhibits apoptosis via AKT (Nikoletopoulou et al. 2013). In cancer treatment, it is intended to prevent necro(pto)sis and rather induce apoptosis. Although necro(pto)sis also results in (cancer) cell death, it is associated with inflammation (Rock, Kono 2008). Inflammation systemically induces fever which can lead to complications, especially in immune-compromised cancer patients (Johnson 1996). The effect of necro(pto)sis induced inflammation on cancer progression is still under debate. On the one hand, the release of inflammatory cytokines can induce moderate inflammation. This recruits the immune system and thus promotes the clearance of cancer cells (Proskuryakov, Gabai 2010). On the other hand, it was described that inflammatory microenvironment might facilitates tumor development by stimulating angiogenesis and metastasis formation (Mantovani et al. 2008). However, different drugs are used although they are known to induce necro(pto)sis, namely arsentic trioxide or tamoxifen (Proskuryakov, Gabai 2010). Advantage of those agents is that they are also efficient in tumor cells that are resistant to apoptosis (Proskuryakov, Gabai 2010). Acquisition of apoptosis resistance is believed to be one of the main mechanisms in development of chemotherapy resistance of cancer cells (Su et al. 2016). This can either be achieved by disrupted apoptosis machinery, overactivated prosurvival signaling pathways, increased expression of the therapeutic target or activation of alternative compensatory pathways (Holohan et al. 2013). 
Up to now, no other group reported that amygdalin increases necrosis in tumor tissue. Cyanide is believed to be the active compound in amygdalin. It is known to have cell toxic properties and thus induce either apoptosis or necro(pto)sis depending on its concentration (Li et al. 2005; Prabhakaran et al. 2002). As described above, cyanide is a cytotoxin that inhibits the mitochondrial enzyme cytochrome oxidase. Inhibition of cytochrome oxidase interrupts the respiratory chain and leads to oxidative stress by increased release of DNAdamaging ROS molecules (Way 1984). This oxidative stress leads to a collapse of the mitochondrial inner transmembrane potential and finally to the loss of mitochondrial membrane integrity (Kroemer et al. 1998). Two consequences of mitochondrial dysfunction are induced. First, bioenergetic catastrophe leads to disruption of cell membrane integrity, inducing necro(pto)sis. Second, activation of caspases by mitochondrial proteins that leak into the cytosol (cytochrome c, apoptosis-inducing factor) inducing apoptosis. Both processes occur in parallel and the relative rate of necro(pto)sis versus apoptosis induction as well as the ATP concentration determines whether a cell will undergo primary necro(pto)sis or apoptosis (Fig.4.8) (Kroemer et al. 1998).

Level of oxidative stress

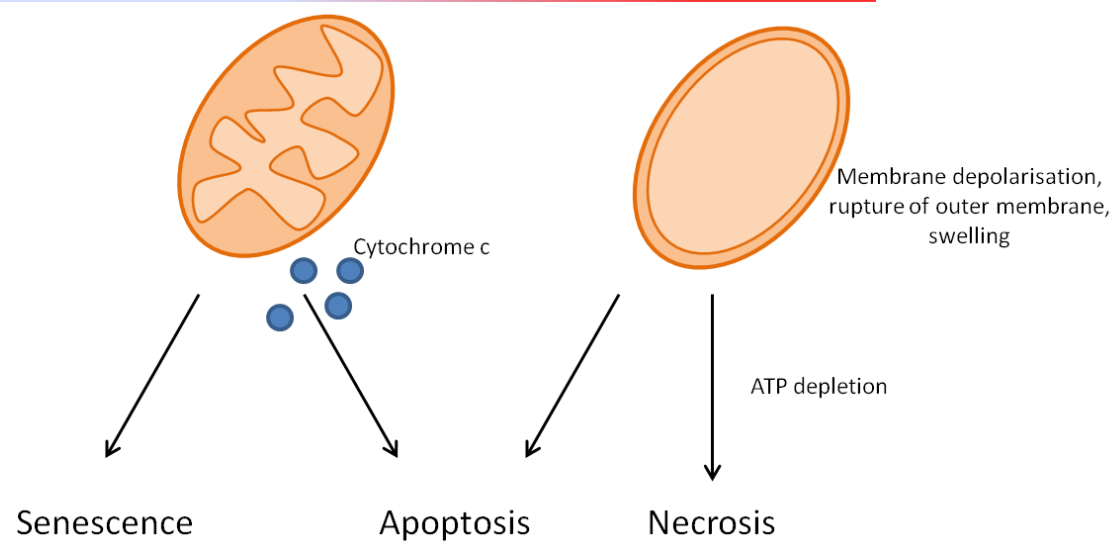

Figure 4.8: Role of mitochondria in oxidative stress response. With increasing level of oxidative stress, the membrane integrity and maintenance of mitochondrial function is impaired. When ROS concentration becomes too high, p53 is induced and initiates senescence or apoptosis induction. If membrane integrity of mitochondria is impaired, cytochrome $\mathrm{c}$ is released and induces apoptosis. Massive oxidative stress ruptures mitochondrial membrane and initiates mitochondrial swelling. Apoptosis or necro(pto)sis is initiated dependent on e.g. ATP concentration. Modified according to Yen, Klionsky (2008) and Levine, Oren (2009).

\subsubsection{How could amygdalin mediates its anti-cancerous effects?}

Cancer treatment can target different pathways or functions of cells with the aim to induce cell cycle arrest, senescence, apoptosis or necro(pto)sis. Amygdalin was long associated 
with cyanide intoxication and described as a toxic substance. Here, we found that parenteral administered amygdalin did not induce toxic side effects. Moreover, amygdalin treatment reduced cancer cell invasiveness and increased necrosis formation in in vivo experiments using the TRAMP-FVB mouse model. A reduced invasiveness, decreased cell proliferation rate and increased senescence induction upon amygdalin treatment was observed in in vitro experiments. The question arises if amygdalin has different effects in vivo and in vitro or if necrosis and senescence are connected and induced by the same mechanism of action. Different mechanisms potentially connect DNA damage, senescence and necrosis. Three possible mechanisms could explain how amygdalin could mediate its effects.

First, mitochondria seem to be a central site of action in amygdalin-mediated cancer treatment. As described above, the inhibition of the cytochrome oxidase by cyanide can inhibit the respiratory chain and release ROS in cases of weaker intoxication and induces mitochondrial swelling and rupture in cases of severe intoxication. Therefore, both, DNA damage-induced senescence as well as necrosis can result from cytochrome oxidase inhibition.

Second, DNA damage can also not only induce senescence but apoptosis and necrosis as well (Borges et al. 2008). Interestingly, Seluanov et al. (2001) described that in response to cellular stress, senescent cells induced necrosis but not apoptosis. Meaning that it is reasonable that also in vivo first senescence occurs and with persistent oxidative stress necrosis is induced. Especially when considered that mice received long term treatment while cell culture was treated for a maximum of seven days, it seems reasonable that the observed necrosis in vivo might occur in succession of senescence. However, if senescence is induced in vivo could not be clarified in the present study.

Third, the ROS which are released due to inhibition of cytochrome oxidase can induce another major player of cell signaling, namely the tumor suppressor p53. Indeed, p53 is involved in the induction of apoptosis, senescence and necrosis (Polager, Ginsberg 2009; te Poele et al. 2002; Wiman 2013). The tumor suppressor p53 is long known to regulate the transcription of a number of genes involved in apoptosis induction and cell cycle regulation. For induction of apoptosis, activated p53 facilitates the transcription of the proapoptotic factors Bid (BH3 interacting-domain death agonist), Bax (B-cell lymphoma 2-associated $\mathrm{X}$ ), Noxa and Puma (Fridman, Lowe 2003). Induction of these factors lead to a release of mitochondrial cytochrome $c$ and therefore triggers apoptosis (Haupt et al. 2003). Senescence is induced by p53-induced expression of p21, PML (promyelocytic leukemia protein) and others (Qian, Chen 2013). The role of p53 in necrosis is not that clear. Accumulation of p53 could facilitate mitochondrial permeability transition pore opening (Vaseva et al. 2012). Degradation of mitochondria initiates a PARP-mediated bioenergetic catastrophe and thus triggers necrosis (Elkholi, Chipuk 2014). Another paper reports p53 activates cathepsin expression and this initiates necrosis (Tu et al. 2009). Furthermore, p53 is also involved in 
the inhibition of invasion and metastasis formation (Bieging et al. 2014), which was observed in amygdalin-treated PCa in vitro and in vivo. p53 inhibits cell invasion by activation of the E3 ubiquitin ligase mouse double minute 2 (MDM2) and thus induces the degradation of key regulators in EMT (Muller et al. 2011) (Fig. 4.9).

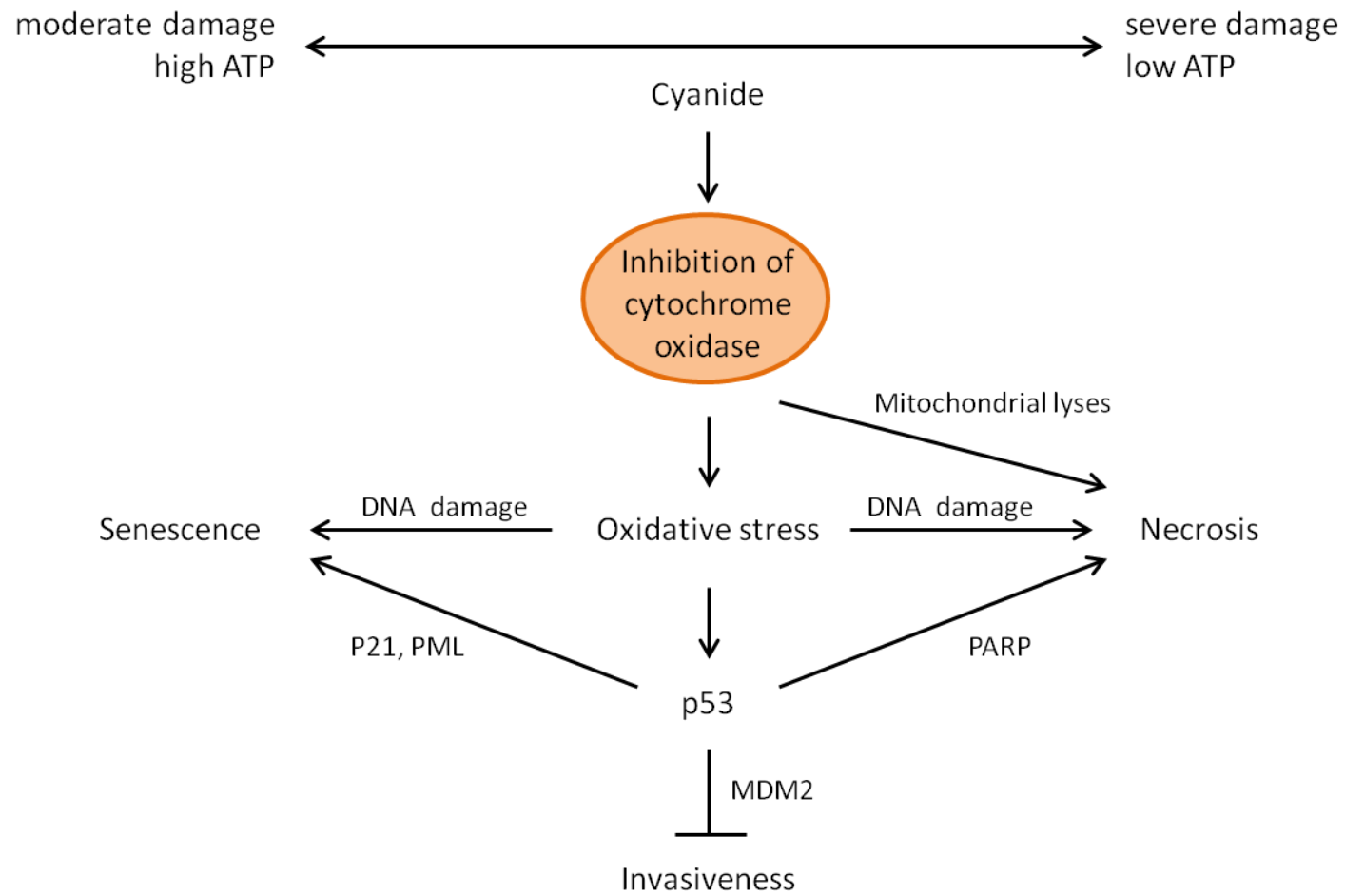

Figure 4.9: Cellular response to cyanide intoxication. Cyanide intoxication inhibits the cytochrome oxidase and therefore triggers mitochondrial lyses, DNA damage and p53 activation. Mitochondrial lyses directly leads to necrosis by the induction of bioenergetic catastrophe. DNA damage can induces either senescence or necrosis, dependent on the severity of damage and the ATP concentration. The tumor suppressor p53 acts as a major regulator of many cellular processes. Upon oxidative stress p53 induces p21 and PML-mediated senescence or PARP-mediated necrosis. Furthermore, activation of p53 induced MDM2-mediated inhibition of cancer cell invasion. PML: promyelocytic leukemia protein; MDM2: mouse double minute 2; PARP: Poly (ADP-ribose) polymerase. Modified according to Polager, Ginsberg (2009), Wiman (2013), te Poele et al. (2002) and Muller et al. (2011).

Taken together, the present study showed that amygdalin exhibit anti-cancerous potential in PCa. We observed induction of senescence in vitro and necrosis in vivo. Invasiveness was reduced in vitro and preventive amygdalin treatment reduced the incidence of distant metastasis formation in vivo. However, the metabolism of amygdalin and the mechanism of action is not fully understood and more research is needed to elucidate how amygdalin induces necrosis in PCa cells but not in other tissues. Here, we suggest three possible mechanisms connecting the effects of amygdalin observed in vitro and in vivo. First, cyanideinduced mitochondrial damage induces dependent on its intensity DNA damage and senescence (mild damage) or necrosis (severe damage). Second, senescence as well as necrosis could be a consequence of cyanide-induced DNA damage (Vakifahmetoglu et al. 
2008). Possibly, first senescence is induced and necrosis results from persistent oxidative stress on the senescent cells (Seluanov et al. 2001). Third, the in vivo observed necrosis and the in vitro observed DNA damage and senescence are both consequences of p53activation. Activation of p53 could furthermore be connected to the in vitro and in vivo observed reduced invasiveness of PCa cells. Indeed, most likely all three mechanisms occur.

\subsection{Perspectives}

\subsubsection{ER $\beta$ in PCa treatment}

The investigation of the role of ER $\beta$ in PCa development and progression is limited by the accuracy of ER $\beta$ expression analysis. Many supposed specific antibodies are available, but still no consensus about the expression of ER $\beta$ in PCa could be found (Christoforou et al. 2014; Dey et al. 2014). In the present study, contradictory results between ER $\beta$ mRNA and protein expression were found. Therefore, better analysis tools to estimate ER $\beta$ expression are needed. Only when reliable data about the expression status of ER $\beta$ in PCa could be generated, reliable studies about ER $\beta$-mediated signaling could be made.

Another major issue during the investigation of the role of ERs in PCa was associated with the TRAMP mouse model. As described above, TRAMP mice developed SGCa with high incidences. SGCa in humans are very rare (Tarjan et al. 2009) and in TRAMP mice SGCa occurs as a side effect of the SV40 T/t antigene overexpression (Tani et al. 2005). However, the massive growth of SGCa resulted in the earlier termination of the experiments. Other PCa mouse models, i.e. the PTEN mouse model could reduce undesired side effects and therefore offers the possibility to analyze the prostate and prostate cancer development over longer time periods.

Most studies claimed that prostate cancer cells exhibit only low ER $\beta$ expression (Dey et al. 2014; Christoforou et al. 2014). The epigenetic silencing of ER $\beta$ expression during PCa progression is one argument for the identification of ER $\beta$ as a tumor suppressor in $\mathrm{PCa}$ (Stettner et al. 2007). Important studies on the role of ER $\beta$ in PCa therefore use artificial overexpression of ER $\beta$ together with ER $\beta$ agonist treatment to show how ER $\beta$ activation impairs signaling in PCa cells (Dey et al. 2014). Since overexpression experiments are susceptible for artificial effects and moreover not applicable in patients, other methods to induce ER $\beta$ re-expression are needed. It could be shown that treatment with DNA methlytransferase inhibitors and histone deacetylase inhibitors induces the expression of ER $\beta$ in advanced PCa (Walton et al. 2008). In this way, the induced re-expression of ER $\beta$ in $\mathrm{PCa}$ is sufficient to reduce PCa proliferation and induce apoptosis (Stettner et al. 2007; Walton et al. 2008). Therefore, it would be interesting if a combined treatment with DNA methlytransferase inhibitors and histone deacetylase inhibitors together with ER $\beta$ agonists could further improve treatment effects. 
The molecular signaling mediating $8 \beta$-VE2-induced treatment effects seems to be ER $\beta$ independent. However, the exact mechanism could not be fully elucidated in the present study. It should be considered that the whole mRNA-sequencing analysis did not give information about RNA without polyadenylation (in consequence of the usage of oligo-dT primers for library generation). Therefore, we cannot make assertions about the potential involvement of those regulatory RNAs. Questions concerning the role of the cholesterol synthesis pathway and AR signaling remain unsolved. It could be shown that several enzymes involved in the cholesterol synthesis pathway are overexpressed upon $8 \beta-V E 2$ treatment and that this effect is not induced by inhibition of P450scc. However, as described above the cholesterol synthesis pathway is involved in PCa progression and therefore, the deregulation of cholesterol synthesis pathway upon $8 \beta$-VE2 treatment might be an important factor. It is unclear, whether the upregulation of the cholesterol synthesis pathway is a direct effect, induced by loss of feedback inhibition because cholesterol is depleted or a consequence of induction of other pathways. Indeed, the cholesterol synthesis pathway is known to be regulated by PI3K and AR signaling in PCa (Krycer, Brown 2013). To get more insights into the processes a measurement of cholesterol and steroid hormones in the cells and the medium could be performed. If cholesterol concentration is high, this would indicate that cholesterol synthesis is used to promote survival in $8 \beta$-VE2-treated cells. Low cholesterol concentration would indicate either cholesterol or cholesterol precursor depletion or inhibition of another enzyme involved in the synthesis process.

The observation that $8 \beta$-VE2 treatment reduced cell survival and induced apoptosis in the AR-positive cell line VCaP but not in AR-negative PC3 cells indicates that the 8$\beta$-VE2mediated effects rely on AR activation. Similar effects were observed for HE3235, which was also only effective in AR-positive cancer cells (Trauger et al. 2009). Performing further experiments with HE3235 could help to elucidate which mechanisms are induced. In order to prove that AR activation is essential for $8 \beta$-VE2-induced effects, PC3 cells with artificial AR expression should be treated with $8 \beta$-VE2 and cell survival, apoptosis induction and gene expression should be investigated. Furthermore, the results from the present study suggest that the inhibition of an alternative AR-mediated survival pathway could be the underlying mechanism mediating $8 \beta$-VE2 treatment effects. Here, Wnt/ $\beta$-catenin signaling and EGF/EGFR signaling were reported to be potential mediators of the alternative survival pathway. Investigation of this hypothesis should be performed by combined treatment of VCaP cells with $8 \beta$-VE2 and Wnt, $\beta$-catenin or EGFR inhibitors. The effects of Wnt, $\beta$-catenin or EGFR inhibition on cell survival, proliferation and gene expression should be investigated.

\subsubsection{Amygdalin in PCa treatment}

This study showed that the alternative cancer drug amygdalin potentially induces anticancerous effects. However, it became clear that one of the main obstacles in amygdalin 
treatment is that it is still not fully understood how amygdalin is metabolized and how it should be applied. In the present work it could be demonstrated that parenteral administration of amygdalin did not induce toxic side effects up to concentrations of $100 \mathrm{mg} / \mathrm{kg}$ amygdalin five times a week. Nonetheless, it was not proven that amygdalin is bioavailable and metabolized by PCa cells. In vivo experiments could show if amygdalin enters tumor tissue or accumulates in any organ by application of radioactive or fluorescence-tagged amygdalin.

It needs to be addressed if and how amygdalin selectively can affect tumor cells. The present study described that $\beta$-glucosidase and rhodanase expression alone could not explain the supposed tumor cell specificity. Moon et al. (2015) reported that rather increased enzyme activity than increased expression leads to tumor cell selective effects of amygdalin. To investigate if amygdalin can potentially be metabolized in PCa cells, an in vitro study could be performed, i.e. cyanide concentration could be measured in medium of amygdalin-treated $\mathrm{PCa}$ and fibroblast cells. Furthermore, the inhibition of telomerase upon amygdalin treatment was demonstrated (Moon et al. 2015). The experiments performed by Moon et al. (2015) described effects on different cancer cell lines including lung cancer, breast cancer and glioblastoma. Therefore, it would be interesting to investigate if the effect described by Moon et al. (2015) also occurs in PCa cells. Inhibition of telomerase activity should induce telomere shortening and thus could be responsible for the induction of senescence. Shortened telomeres could be detected in vitro and in vivo by FISH analysis. Incubation with telomere specific probes should give information about telomere size. Moreover, the expression of PARP, p21 and p53 should be investigated to elucidate the mechanism of senescence and necrosis induction. Analysis of the expression of $\beta 1$ and $\beta 4$ integrin could reveal information about the molecular signaling events underlying the reduced invasive potential of PCa cells upon amygdalin treatment. The degradation of protein quality during amygdalin treatment hinders such investigations in vitro and in vivo. The induction of necrosis furthermore impedes signaling analysis in prostate tumor tissue. Therefore, it would be reasonable to terminate in vivo experiments at an earlier time point, because in non-advanced prostate tumors necrosis might not be progressed, and therefore, differences in cell signaling could be detected. However, adequate timing is mandatory since in vitro analysis showed that amygdalin induces effects only after longer periods of treatment. To further elucidate the underlying mechanisms of amygdalin action in the TRAMP-FVB mouse model, in vivo analysis of induction of senescence in prostate tumors should be performed. This could give information whether necrosis is induced as a consequence of oxidative stress in senescent tumor cells or mediated by a senescence-independent mechanism. 


\section{Summary}

\subsection{The role of ER $\beta$ and effects of $8 \beta-V E 2$ treatment in $\mathrm{PCa}$}

The key pathway deregulated in $\mathrm{PCa}$ is the androgen receptor (AR) signaling pathway (Lonergan, Tindall 2011). Different attempts to inhibit AR signaling by ligand synthesis inhibition or blockade of the ligand binding site failed to be curative, and cancer cells developed different adaptation mechanisms (Knudsen, Penning 2010). Therefore, the targeting of other pathways involved in PCa became a new field of research. The signaling of the estrogen receptors (ERs) was suggested to have an impact of PCa development and progression (Christoforou et al. 2014). Whereas ER a was reported to promote PCa growth (Ricke et al. 2008), ER $\beta$ is described to have a tumor suppressive function in PCa (Horvath et al. 2001). However, to date little is known about ER signaling in PCa. The present study aimed to investigate how ER signaling regulates $\mathrm{PCa}$ development and growth and if ER $\beta$ activation is a potential treatment opportunity for different stages of PCa. The function of ERs should be investigated in cell lines derived from PCa of TRAMP mice and TRAMP mice with either an ER $\alpha$ knockout (ERKO) or an ER $\beta$ knockout (BERKO). ERKO/TRAMP mice developed PIN lesions but no PCa which correlates with the suggested tumor promoting function of ER $\alpha$. The massive growth of SGCa made early termination of ERKO/TRAMP experiments necessary, so it could not be estimated if PCa occurs in ERKO/TRAMP mice at older ages. Since no PCa were observed, no primary ERKO/TRAMP PCa cell line could be generated. In contrast, both, TRAMP and BERKO/TRAMP, developed PCa with comparable incidence and malignancies. Cell lines from TRAMP and BERKO/TRAMP PCas showed similar proliferation rates. No tumor suppressive effect of ER $\beta$ could be found. However, it was unclear if BERKO/TRAMP mice indeed exhibit a complete ER $\beta$ knockout as indicated by genotyping and sequencing analysis, or express ER $\beta$ or an ER $\beta$ splice variant as indicated by protein analysis. The findings of the present study differ from the literature Slusarz et al. (2012) and therefore more research is needed to elucidate if the described mouse models are suitable of ER studies.

In order to mimic different PCa stages, the human PCa cell line VCaP was treated on the one hand with testosterone to generate androgen-dependent VCaP rev. cells and on the other hand with the agent abiraterone acetate to generate VCaP AA cells under maximum ADT. VCaP rev. cells showed low AR and PSA expression compared to VCaP cells. VCaP AA cells exhibited increased expression of AR splice variants, indicating advanced resistance to ADT. Upon treatment with the ER $\beta$ agonist $8 \beta$-VE2 all three VCaP cell variants showed cell survival reduction and apoptosis induction. Moreover, the expression of the AR and ARVs was downregulated in VCaP and VCaP AA cells after $8 \beta$-VE2 treatment, whereas the expression of AR-regulated genes was increased. However, VCaP rev. cells showed increased AR expression but decreased AR target gene expression. Whole mRNA- 
sequencing analysis of differential gene expression could not identify any connection to commonly known ER $\beta$ signaling. Moreover, inhibition of ER $\beta$ signaling could not block the $8 \beta$-VE2-induced treatment effects. We thereby concluded that the observed effects of $8 \beta$ VE2 treatment of VCaP cell variants were not solely ER $\beta$-mediated. Indeed, another pathway might be responsible for the observed effects. Whole mRNA-sequencing analysis showed deregulation of the cholesterol synthesis pathway and PI3K signaling in 8 $\beta$-VE2treated PCa cells. Inhibition of the cholesterol synthesis pathway as well as inhibition or activation of PI3K signaling could be excluded as the underlying cause for $8 \beta$-VE2-mediated effects. Overlapping results obtained from the $8 \beta$-VE2-induced expression profile and the DHT-induced expression profile of $\mathrm{VCaP}$ cells point to an activation of the AR signaling pathway. Furthermore, $8 \beta$-VE2 failed to induce any effects in AR-negative PC3 cells. However, no AR-specific transcriptional activity could be found in $8 \beta$-VE2-treated VCaP cells. Possible explanations for these effects could be the induction of the membrane-bound AR signaling pathway. Here, AR does not directly act as a transcription factor but initiates a signaling cascade, inducing the activation of gene transcription and cell survival regulation factors. In fact, the observed decrease of cell survival and the induction of apoptosis upon $8 \beta$-VE2 treatment did not answer the expectations of $\mathrm{AR}$ activation in PCa cells. By recruitment of $A R$ coregulators, the gene expression can be regulated, e.g. the coregulatordependent auto-regulation of $A R$ was well-described by Cai et al. (2011). The AR is suggested to mediate two main pathways. First, it regulates gene expression involved in prostate cell maintenance and growth. Second, it was hypothesized that AR signaling regulates an alternative survival pathway. This alternative survival pathway is inhibited by AR activation in normal prostate cells but during PCa progression the loss of inhibition promotes PCa growth (Karantanos et al. 2013). It is unknown which signaling might mediate this alternative survival pathway. Possible candidates are Wnt/ $\beta$-catenin signaling and EGF/EGFR signaling, which are supported by the whole mRNA-sequencing data.

In summary, treatment of different PCa stages with $8 \beta$-VE2 led to efficient cell survival reduction and induction of apoptosis. $8 \beta$-VE2 treatment downregulated $A R$ and $A R V$ expression and thereby reverses the adaptation to ADT. However, ER $\beta$ activation is not likely to be responsible for these effects. More likely, the effects in $8 \beta$-VE2-treated VCaP cells could be induced by the restoration of the full AR signaling, i.e. activation of $A R$ gene transcription, for example by membrane-bound AR signaling and the inhibition of an ARregulated alternative survival pathway. Further examination of the $\mathrm{Wnt} / \beta$-catenin signaling and EGF/EGFR signaling pathways could help to elucidate if $\mathrm{PCa}$ cell survival promoting pathways are inhibited by $8 \beta$-VE2 treatment. $8 \beta$-VE2 was identified as an effective anticancerous drug, decreasing $\mathrm{PCa}$ cell survival, $\mathrm{AR}$ and $\mathrm{ARV}$ expression and inducing apoptosis. However, further studies are needed to elucidate the underlying molecular mechanism of action. 


\subsection{Amygdalin in PCa treatment}

The natural occurring cyanogenic diglucoside amygdalin, found in seeds of different rosacea, was long believed to have therapeutic potential in different diseases, e.g. asthmatic, pulmonary fibrosis, hyperoxia induced lung injury, immune regulation, etc (Song, Xu 2014). Cyanide was described to be the active component of amygdalin. Different studies disprove therapeutic use but emphasize toxic properties of amygdalin. However, current investigations underpin that amygdalin indeed has cancer cell toxic properties. In vitro studies on PCa cell lines showed that amygdalin treatment induced cell cycle arrest and apoptosis (Chang et al. 2006; Makarevic et al. 2016). The present study aimed to elucidate the effect of amygdalin on PCa growth in vivo. Therefore, TRAMP-FVB mice were treated with different amygdalin concentrations from six weeks of age (preventive treatment group) or 16 weeks of age (curative treatment group) on. Up to now, no consensus could be found about the question how amygdalin should be administered. Here, we showed that in mouse PCa tissue the enzyme $\beta$-glucosidase, presumably needed for metabolisation of amygdalin, is expressed. Therefore, parenteral administration of amygdalin, preventing systemic cyanide intoxication was chosen. The survival time of mice did not change due to the amygdalin treatment, but the preventive treatment group showed reduced formation of metastasis under treatment with high amygdalin concentrations compared to control mice. Furthermore, $100 \mathrm{mg} / \mathrm{kg}$ amygdalin increased the formation of necrotic tumor tissue in preventive- and curativetreated TRAMP-FVB mice compared to untreated control mice. Due to the massive induction of necrosis, no further analysis of cell signaling could be performed on the tumor tissue sections or tumor proteins. Therefore, analyses concerning the underlying mechanisms of amygdalin-induced effects were performed on murine primary PCa cell lines T244 and 2E. Amygdalin induced a reduction of cell survival but without the induction of apoptosis or cytotoxic effects. Moreover, amygdalin treatment reduced invasiveness of T244 cells. Cell morphological changes and increased expression of senescence associated $\beta$-gal (SA $\beta$-gal) showed that senescence was induced in amygdalin-treated cell lines. As potential causes for senescence induction mitotic catastrophe and induction of DNA double strand breaks could be identified. Senescence as well as necrosis can both be induced upon cyanide release. Taken together, this study supports the finding that amygdalin has anti-cancerous potential, most likely mediated by cyanide release. In the present study amygdalin does not induce toxic side effects. The release of cyanide was described to induce dependent on its concentration either senescence or necrosis due to inhibition of the respiratory chain and induction of DNA damage. Thus, for the first time the present study provides evidence-based indications for amygdalin's anti-cancerous potential in PCa treatment in vivo. 


\section{Publication bibliography}

Abbas, Ata; Gupta, Sanjay (2008): The role of histone deacetylases in prostate cancer. In Epigenetics 3 (6), pp. 300-309.

Adams, Peter D. (2009): Healing and hurting: molecular mechanisms, functions, and pathologies of cellular senescence. In Molecular cell 36 (1), pp. 2-14. DOI: 10.1016/j.molcel.2009.09.021.

Ahlem, Clarence; Kennedy, Michael; Page, Theodore; Bell, David; Delorme, Evelyn; Villegas, Sonia et al. (2012): 17alpha-alkynyl 3alpha, 17beta-androstanediol nonclinical and clinical pharmacology, pharmacokinetics and metabolism. In Investigational new drugs 30 (1), pp. 59-78. DOI: 10.1007/s10637-010-9517-0.

Ahuja, Deepika; Saenz-Robles, M. Teresa; Pipas, James M. (2005): SV40 large T antigen targets multiple cellular pathways to elicit cellular transformation. In Oncogene 24 (52), pp. 7729-7745. DOI: 10.1038/sj.onc.1209046.

Allsopp, R. C.; Chang, E.; Kashefi-Aazam, M.; Rogaev, E. I.; Piatyszek, M. A.; Shay, J. W.; Harley, C. B. (1995): Telomere shortening is associated with cell division in vitro and in vivo. In Experimental cell research 220 (1), pp. 194-200. DOI: 10.1006/excr.1995.1306.

Antonarakis, Emmanuel S.; Lu, Changxue; Wang, Hao; Luber, Brandon; Nakazawa, Mary; Roeser, Jeffrey C. et al. (2014): AR-V7 and resistance to enzalutamide and abiraterone in prostate cancer. In The New England journal of medicine 371 (11), pp. 1028-1038. DOI: 10.1056/NEJMoa1315815.

Arora, Vivek K.; Schenkein, Emily; Murali, Rajmohan; Subudhi, Sumit K.; Wongvipat, John; Balbas, Minna D. et al. (2013): Glucocorticoid receptor confers resistance to antiandrogens by bypassing androgen receptor blockade. In Cell 155 (6), pp. 13091322. DOI: 10.1016/j.cell.2013.11.012.

Asuthkar, Swapna; Elustondo, Pia A.; Demirkhanyan, Lusine; Sun, Xiaohui; Baskaran, Padmamalini; Velpula, Kiran Kumar et al. (2015): The TRPM8 protein is a testosterone receptor: I. Biochemical evidence for direct TRPM8-testosterone interactions. In The Journal of biological chemistry 290 (5), pp. 2659-2669. DOI: 10.1074/jbc.M114.610824.

Balkon, J. (1982): Methodology for the detection and measurement of amygdalin in tissues and fluids. In Journal of analytical toxicology 6 (5), pp. 244-246.

Barascu, Aurelia; Le Chalony, Catherine; Pennarun, Gaelle; Genet, Diane; Imam, Naima; Lopez, Bernard; Bertrand, Pascale (2012): Oxidative stress induces an ATM- 
independent senescence pathway through p38 MAPK-mediated lamin B1 accumulation. In The EMBO journal 31 (5), pp. 1080-1094. DOI: 10.1038/emboj.2011.492.

Barry Delongchamps, N. (2014): Prostate cancer: review in 2014. In Diagnostic and interventional imaging 95 (7-8), pp. 739-742. DOI: 10.1016/j.diii.2014.06.005.

Bayreuther, K.; Rodemann, H. P.; Hommel, R.; Dittmann, K.; Albiez, M.; Francz, P. I. (1988): Human skin fibroblasts in vitro differentiate along a terminal cell lineage. In Proceedings of the National Academy of Sciences of the United States of America 85 (14), pp. 5112-5116.

Beer, Tomasz M.; Lemmon, Dianne; Lowe, Bruce A.; Henner, W. David (2003): High-dose weekly oral calcitriol in patients with a rising PSA after prostatectomy or radiation for prostate carcinoma. In Cancer 97 (5), pp. 1217-1224. DOI: 10.1002/cncr.11179.

Bernard, David; Pourtier-Manzanedo, Albin; Gil, Jesus; Beach, David H. (2003): Myc confers androgen-independent prostate cancer cell growth. In The Journal of clinical investigation 112 (11), pp. 1724-1731. DOI: 10.1172/JCI19035.

Bieging, Kathryn T.; Mello, Stephano Spano; Attardi, Laura D. (2014): Unravelling mechanisms of p53-mediated tumour suppression. In Nature reviews. Cancer 14 (5), pp. 359-370. DOI: 10.1038/nrc3711.

Bitting, Rhonda L.; Armstrong, Andrew J. (2013): Targeting the PI3K/Akt/mTOR pathway in castration-resistant prostate cancer. In Endocrine-related cancer 20 (3), R83-99. DOI: 10.1530/ERC-12-0394.

Blagosklonny, Mikhail V. (2003): Cell senescence and hypermitogenic arrest. In EMBO reports 4 (4), pp. 358-362. DOI: 10.1038/sj.embor.embor806.

Boehm, Jesse S.; Hahn, William C. (2005): Understanding transformation: progress and gaps. In Current opinion in genetics \& development 15 (1), pp. 13-17. DOI: 10.1016/j.gde.2004.11.003

Bolarinwa, Islamiyat F.; Orfila, Caroline; Morgan, Michael R. A. (2014): Amygdalin content of seeds, kernels and food products commercially-available in the UK. In Food chemistry 152, pp. 133-139. DOI: 10.1016/j.foodchem.2013.11.002.

Bolton, Eric C.; So, Alex Y.; Chaivorapol, Christina; Haqq, Christopher M.; Li, Hao; Yamamoto, Keith R. (2007): Cell- and gene-specific regulation of primary target genes by the androgen receptor. In Genes \& development 21 (16), pp. 2005-2017. DOI: $10.1101 / \mathrm{gad} .1564207$. 
Borges, Helena Lobo; Linden, Rafael; Wang, Jean Y. J. (2008): DNA damage-induced cell death: lessons from the central nervous system. In Cell research 18 (1), pp. 17-26. DOI: $10.1038 / \mathrm{cr} .2007 .110$.

Bosland, M. C.; Ford, H.; Horton, L. (1995): Induction at high incidence of ductal prostate adenocarcinomas in NBL/Cr and Sprague-Dawley Hsd:SD rats treated with a combination of testosterone and estradiol-17 beta or diethylstilbestrol. In Carcinogenesis 16 (6), pp. 1311-1317.

Bradford, M. M. (1976): A rapid and sensitive method for the quantitation of microgram quantities of protein utilizing the principle of protein-dye binding. In Analytical biochemistry 72, pp. 248-254.

Bu, Huajie; Bormann, Stefanie; Schafer, Georg; Horninger, Wolfgang; Massoner, Petra; Neeb, Antje et al. (2011): The anterior gradient 2 (AGR2) gene is overexpressed in prostate cancer and may be useful as a urine sediment marker for prostate cancer detection. In The Prostate 71 (6), pp. 575-587. DOI: 10.1002/pros.21273.

Bubendorf, L.; Schopfer, A.; Wagner, U.; Sauter, G.; Moch, H.; Willi, N. et al. (2000): Metastatic patterns of prostate cancer: an autopsy study of 1,589 patients. In Human pathology 31 (5), pp. 578-583.

Burd, Craig J.; Morey, Lisa M.; Knudsen, Karen E. (2006): Androgen receptor corepressors and prostate cancer. In Endocrine-related cancer 13 (4), pp.979-994. DOI: 10.1677/erc. 1.01115 .

Burton, Dominick G. A.; Giribaldi, Maria G.; Munoz, Anisleidys; Halvorsen, Katherine; Patel, Asmita; Jorda, Merce et al. (2013): Androgen deprivation-induced senescence promotes outgrowth of androgen-refractory prostate cancer cells. In PloS one 8 (6), e68003. DOI: 10.1371/journal.pone.0068003.

Buttigliero, Consuelo; Tucci, Marcello; Bertaglia, Valentina; Vignani, Francesca; Bironzo, Paolo; Di Maio, Massimo; Scagliotti, Giorgio Vittorio (2015): Understanding and overcoming the mechanisms of primary and acquired resistance to abiraterone and enzalutamide in castration resistant prostate cancer. In Cancer treatment reviews 41 (10), pp. 884-892. DOI: 10.1016/j.ctrv.2015.08.002.

Cai, Changmeng; He, Housheng Hansen; Chen, Sen; Coleman, Ilsa; Wang, Hongyun; Fang, $\mathrm{Zi}$ et al. (2011): Androgen receptor gene expression in prostate cancer is directly suppressed by the androgen receptor through recruitment of lysine-specific demethylase 1. In Cancer cell 20 (4), pp. 457-471. DOI: 10.1016/j.ccr.2011.09.001.

Campisi, Judith (2013): Aging, cellular senescence, and cancer. In Annual review of physiology 75, pp. 685-705. DOI: 10.1146/annurev-physiol-030212-183653. 
Carter, J. H.; McLafferty, M. A.; Goldman, P. (1980): Role of the gastrointestinal microflora in amygdalin (laetrile)-induced cyanide toxicity. In Biochemical pharmacology 29 (3), pp. 301-304.

Chang, Hyun-Kyung; Shin, Mal-Soon; Yang, Hye-Young; Lee, Jin-Woo; Kim, Young-Sick; Lee, Myoung-Hwa et al. (2006): Amygdalin induces apoptosis through regulation of Bax and Bcl-2 expressions in human DU145 and LNCaP prostate cancer cells. In Biological \& pharmaceutical bulletin 29 (8), pp. 1597-1602.

Chang, Li-wen; Zhu, Hua-ping; Li, Wen-bin; Liu, Han-chu; Zhang, Qian-shen; Chen, Hongbing (2005): Protective effects of amygdalin on hyperoxia-exposed type II alveolar epithelial cells isolated from premature rat lungs in vitro. In Zhonghua er ke za zhi= Chinese journal of pediatrics 43 (2), pp. 118-123.

Chen, Charlie D.; Welsbie, Derek S.; Tran, Chris; Baek, Sung Hee; Chen, Randy; Vessella, Robert et al. (2004): Molecular determinants of resistance to antiandrogen therapy. In Nature medicine 10 (1), pp. 33-39. DOI: 10.1038/nm972.

Chen, Jinyao; Song, Yang; Zhang, Lishi (2013): Lycopene/tomato consumption and the risk of prostate cancer: a systematic review and meta-analysis of prospective studies. In Journal of nutritional science and vitaminology 59 (3), pp. 213-223.

Chen, Zhenbang; Trotman, Lloyd C.; Shaffer, David; Lin, Hui-Kuan; Dotan, Zohar A.; Niki, Masaru et al. (2005): Crucial role of p53-dependent cellular senescence in suppression of Pten-deficient tumorigenesis. In Nature 436 (7051), pp. 725-730. DOI: 10.1038/nature03918.

Cheng, Jennifer; Lee, Eun Jig; Madison, Laird D.; Lazennec, Gwendal (2004): Expression of estrogen receptor beta in prostate carcinoma cells inhibits invasion and proliferation and triggers apoptosis. In FEBS letters 566 (1-3), pp. 169-172. DOI: 10.1016/j.febslet.2004.04.025.

Chesire, Dennis R.; Isaacs, William B. (2002): Ligand-dependent inhibition of betacatenin/TCF signaling by androgen receptor. In Oncogene 21 (55), pp. 8453-8469. DOI: $10.1038 /$ sj.onc.1206049.

Cho, Kyung A.; Ryu, Sung Jin; Oh, Yoon Sin; Park, Ji Hyeun; Lee, Jung Weon; Kim, HwangPhill et al. (2004): Morphological adjustment of senescent cells by modulating caveolin1 status. In The Journal of biological chemistry 279 (40), pp. 42270-42278. DOI: 10.1074/jbc.M402352200.

Christoforou, Paraskevi; Christopoulos, Panagiotis F.; Koutsilieris, Michael (2014): The role of estrogen receptor beta in prostate cancer. In Molecular medicine (Cambridge, Mass.) 20, pp. 427-434. DOI: 10.2119/molmed.2014.00105. 
Collado, Manuel; Serrano, Manuel (2010): Senescence in tumours: evidence from mice and humans. In Nature reviews. Cancer 10 (1), pp. 51-57. DOI: 10.1038/nrc2772.

Cooper, R. A. (1978): Influence of increased membrane cholesterol on membrane fluidity and cell function in human red blood cells. In Journal of supramolecular structure 8 (4), pp. 413-430. DOI: 10.1002/jss.400080404.

Couse, J. F.; Lindzey, J.; Grandien, K.; Gustafsson, J. A.; Korach, K. S. (1997): Tissue distribution and quantitative analysis of estrogen receptor-alpha (ERalpha) and estrogen receptor-beta (ERbeta) messenger ribonucleic acid in the wild-type and ERalpha-knockout mouse. In Endocrinology 138 (11), pp. 4613-4621. DOI: 10.1210/endo.138.11.5496.

Cowley, S. M.; Hoare, S.; Mosselman, S.; Parker, M. G. (1997): Estrogen receptors alpha and beta form heterodimers on DNA. In The Journal of biological chemistry 272 (32), pp. 19858-19862.

Cui, Jie; Shen, Yong; Li, Rena (2013): Estrogen synthesis and signaling pathways during aging: from periphery to brain. In Trends in molecular medicine 19 (3), pp. 197-209. DOI: 10.1016/j.molmed.2012.12.007.

Cyster, Jason G.; Dang, Eric V.; Reboldi, Andrea; Yi, Tangsheng (2014): 25Hydroxycholesterols in innate and adaptive immunity. In Nature reviews. Immunology 14 (11), pp. 731-743. DOI: 10.1038/nri3755.

Das, Susobhan; Roth, Cherice P.; Wasson, Lori M.; Vishwanatha, Jamboor K. (2007): Signal transducer and activator of transcription-6 (STAT6) is a constitutively expressed survival factor in human prostate cancer. In The Prostate 67 (14), pp. 1550-1564. DOI: 10.1002/pros.20640.

DeBose-Boyd, Russell A. (2008): Feedback regulation of cholesterol synthesis: sterolaccelerated ubiquitination and degradation of HMG CoA reductase. In Cell research 18 (6), pp. 609-621. DOI: 10.1038/cr.2008.61.

Dey, P.; Strom, A.; Gustafsson, J-A (2014): Estrogen receptor beta upregulates FOXO3a and causes induction of apoptosis through PUMA in prostate cancer. In Oncogene 33 (33), pp. 4213-4225. DOI: 10.1038/onc.2013.384.

Dey, Prasenjit; Jonsson, Philip; Hartman, Johan; Williams, Cecilia; Strom, Anders; Gustafsson, Jan-Ake (2012): Estrogen receptors beta1 and beta2 have opposing roles in regulating proliferation and bone metastasis genes in the prostate cancer cell line PC3. In Molecular endocrinology (Baltimore, Md.) 26 (12), pp. 1991-2003. DOI: 10.1210/me.2012.1227. 
Di d'Adda Fagagna, Fabrizio (2008): Living on a break: cellular senescence as a DNAdamage response. In Nature reviews. Cancer 8 (7), pp.512-522. DOI: 10.1038/nrc2440.

Dimri, G. P.; Lee, X.; Basile, G.; Acosta, M.; Scott, G.; Roskelley, C. et al. (1995): A biomarker that identifies senescent human cells in culture and in aging skin in vivo. In Proceedings of the National Academy of Sciences of the United States of America 92 (20), pp. 9363-9367.

Dimri, Goberdhan P. (2005): What has senescence got to do with cancer? In Cancer cell 7 (6), pp. 505-512. DOI: 10.1016/j.ccr.2005.05.025.

Do, Jeong-Su; Hwang, Jin-Ki; Seo, Hyo-Jung; Woo, Won-Hong; Nam, Sang-Yun (2006): Antiasthmatic activity and selective inhibition of type 2 helper $\mathrm{T}$ cell response by aqueous extract of semen armeniacae amarum. In Immunopharmacology and immunotoxicology 28 (2), pp. 213-225. DOI: 10.1080/08923970600815253.

Dorr, R. T.; Paxinos, J. (1978): The current status of laetrile. In Annals of internal medicine 89 (3), pp. 389-397.

Drexler, Hans G.; Uphoff, Cord C. (2002): Mycoplasma contamination of cell cultures: Incidence, sources, effects, detection, elimination, prevention. In Cytotechnology 39 (2), pp. 75-90. DOI: 10.1023/A:1022913015916.

Edlind, Merritt P.; Hsieh, Andrew C. (2014): PI3K-AKT-mTOR signaling in prostate cancer progression and androgen deprivation therapy resistance. In Asian journal of andrology 16 (3), pp. 378-386. DOI: 10.4103/1008-682X.122876.

Edwards, D. P. (2000): The role of coactivators and corepressors in the biology and mechanism of action of steroid hormone receptors. In Journal of mammary gland biology and neoplasia 5 (3), pp. 307-324.

Elkholi, Rana; Chipuk, Jerry E. (2014): How do I kill thee? Let me count the ways: p53 regulates PARP-1 dependent necrosis. In BioEssays : news and reviews in molecular, cellular and developmental biology 36 (1), pp. 46-51. DOI: 10.1002/bies.201300117.

Elmore, Susan (2007): Apoptosis: a review of programmed cell death. In Toxicologic pathology 35 (4), pp. 495-516. DOI: 10.1080/01926230701320337.

Enmark, E.; Pelto-Huikko, M.; Grandien, K.; Lagercrantz, S.; Lagercrantz, J.; Fried, G. et al. (1997): Human estrogen receptor beta-gene structure, chromosomal localization, and expression pattern. In The Journal of clinical endocrinology and metabolism 82 (12), pp. 4258-4265. DOI: 10.1210/jcem.82.12.4470. 
Eom, Young-Woo; Kim, Mi Ae; Park, Seok Soon; Goo, Mi Jin; Kwon, Hyuk Jae; Sohn, Seonghyang et al. (2005): Two distinct modes of cell death induced by doxorubicin: apoptosis and cell death through mitotic catastrophe accompanied by senescence-like phenotype. In Oncogene 24 (30), pp. 4765-4777. DOI: 10.1038/sj.onc.1208627.

Eskra, Jillian (2015): Culture methods for VCaP prostate cancer cells. In Protocol Exchange. DOI: $10.1038 /$ protex.2015.077.

Fang, Jing; Ding, Min; Yang, Lily; Liu, Ling-Zhi; Jiang, Bing-Hua (2007): PI3K/PTEN/AKT signaling regulates prostate tumor angiogenesis. In Cellular signalling 19 (12), pp. 2487-2497. DOI: 10.1016/j.cellsig.2007.07.025.

Fatima, Roshan; Akhade, Vijay Suresh; Pal, Debosree; Rao, Satyanarayana (2015): Long noncoding RNAs in development and cancer: potential biomarkers and therapeutic targets. In Molecular and cellular therapies 3, p. 5. DOI: 10.1186/s40591-015-0042-6.

Festjens, Nele; Vanden Berghe, Tom; Vandenabeele, Peter (2006): Necrosis, a wellorchestrated form of cell demise: signalling cascades, important mediators and concomitant immune response. In Biochimica et biophysica acta 1757 (9-10), pp. 1371-1387. DOI: 10.1016/j.bbabio.2006.06.014.

Festuccia, C.; Muzi, P.; Millimaggi, D.; Biordi, L.; Gravina, G. L.; Speca, S. et al. (2005): Molecular aspects of gefitinib antiproliferative and pro-apoptotic effects in PTENpositive and PTEN-negative prostate cancer cell lines. In Endocrine-related cancer 12 (4), pp. 983-998. DOI: 10.1677/erc.1.00986.

Ford, O. Harris 3rd; Gregory, Christopher W.; Kim, Desok; Smitherman, Andrew B.; Mohler, James L. (2003): Androgen receptor gene amplification and protein expression in recurrent prostate cancer. In The Journal of urology 170 (5), pp. 1817-1821. DOI: 10.1097/01.ju.0000091873.09677.f4.

Freeman, Michael R.; Cinar, Bekir; Kim, Jayoung; Mukhopadhyay, Nishit K.; Di Vizio, Dolores; Adam, Rosalyn M.; Solomon, Keith R. (2007): Transit of hormonal and EGF receptor-dependent signals through cholesterol-rich membranes. In Steroids 72 (2), pp. 210-217. DOI: 10.1016/j.steroids.2006.11.012.

Freeman, Michael R.; Solomon, Keith R. (2004): Cholesterol and prostate cancer. In Journal of cellular biochemistry 91 (1), pp. 54-69. DOI: 10.1002/jcb.10724.

Freund, Adam; Orjalo, Arturo V.; Desprez, Pierre-Yves; Campisi, Judith (2010): Inflammatory networks during cellular senescence: causes and consequences. In Trends in molecular medicine 16 (5), pp. 238-246. DOI: 10.1016/j.molmed.2010.03.003.

Fridman, Jordan S.; Lowe, Scott W. (2003): Control of apoptosis by p53. In Oncogene 22 (56), pp. 9030-9040. DOI: 10.1038/sj.onc.1207116. 
Gershoni, J. M.; Palade, G. E. (1982): Electrophoretic transfer of proteins from sodium dodecyl sulfate-polyacrylamide gels to a positively charged membrane filter. In Analytical biochemistry 124 (2), pp. 396-405.

Goetzl, M. A.; van Veldhuizen, P. J.; Thrasher, J. B. (2007): Effects of soy phytoestrogens on the prostate. In Prostate cancer and prostatic diseases 10 (3), pp. 216-223. DOI: 10.1038/sj.pcan.4500953.

Gosden, J. R.; Middleton, P. G.; Rout, D. (1986): Localization of the human oestrogen receptor gene to chromosome 6q24----q27 by in situ hybridization. In Cytogenetics and cell genetics 43 (3-4), pp. 218-220.

Graca, Ines; Pereira-Silva, Eva; Henrique, Rui; Packham, Graham; Crabb, Simon J.; Jeronimo, Carmen (2016): Epigenetic modulators as therapeutic targets in prostate cancer. In Clinical epigenetics 8, p. 98. DOI: 10.1186/s13148-016-0264-8.

Greenberg, N. M.; DeMayo, F.; Finegold, M. J.; Medina, D.; Tilley, W. D.; Aspinall, J. O. et al. (1995): Prostate cancer in a transgenic mouse. In Proceedings of the National Academy of Sciences of the United States of America 92 (8), pp. 3439-3443.

Greider, Carol W.; Blackburn, Elizabeth H. (1985): Identification of a specific telomere terminal transferase activity in tetrahymena extracts. In Cell 43 (2), pp. 405-413. DOI: 10.1016/0092-8674(85)90170-9.

Guo, Wenhuan; Keener, Anne L.; Jing, Yifeng; Cai, Liquan; Ai, Junkui; Zhang, Jian et al. (2015): FOXA1 modulates EAF2 regulation of AR transcriptional activity, cell proliferation, and migration in prostate cancer cells. In The Prostate 75 (9), pp. 976987. DOI: 10.1002/pros.22982.

Guo, Wenjun; Giancotti, Filippo G. (2004): Integrin signalling during tumour progression. In Nature reviews. Molecular cell biology 5 (10), pp. 816-826. DOI: 10.1038/nrm1490.

Haisman, D. R.; Knight, D. J. (1967): The enzymic hydrolysis of amygdalin. In The Biochemical journal 103 (2), pp. 528-534.

Hammarsten, Jan; Hogstedt, Benkt (2002): Calculated fast-growing benign prostatic hyperplasia--a risk factor for developing clinical prostate cancer. In Scandinavian journal of urology and nephrology 36 (5), pp.330-338. DOI: 10.1080/003655902320783827.

Hanahan, Douglas; Weinberg, Robert A. (2011): Hallmarks of cancer: the next generation. In Cell 144 (5), pp. 646-674. DOI: 10.1016/j.cell.2011.02.013. 
Haupt, Susan; Berger, Michael; Goldberg, Zehavit; Haupt, Ygal (2003): Apoptosis - the p53 network. In Journal of cell science 116 (Pt 20), pp. 4077-4085. DOI: 10.1242/jcs.00739.

Hayflick, L.; Moorhead, P. S. (1961): The serial cultivation of human diploid cell strains. In Experimental cell research 25, pp. 585-621.

Heidenreich, Axel; Bastian, Patrick J.; Bellmunt, Joaquim; Bolla, Michel; Joniau, Steven; van der Kwast, Theodor et al. (2014): EAU guidelines on prostate cancer. Part II: Treatment of advanced, relapsing, and castration-resistant prostate cancer. In European urology 65 (2), pp. 467-479. DOI: 10.1016/j.eururo.2013.11.002.

Heinlein, Cynthia A.; Chang, Chawnshang (2002a): Androgen receptor (AR) coregulators: an overview. In Endocrine reviews 23 (2), pp. 175-200. DOI: 10.1210/edrv.23.2.0460.

Heinlein, Cynthia A.; Chang, Chawnshang (2002b): The roles of androgen receptors and androgen-binding proteins in nongenomic androgen actions. In Molecular endocrinology (Baltimore, Md.) 16 (10), pp. 2181-2187. DOI: 10.1210/me.2002-0070.

Hers, Ingeborg; Vincent, Emma E.; Tavare, Jeremy M. (2011): Akt signalling in health and disease. In Cellular signalling 23 (10), pp. 1515-1527. DOI: 10.1016/j.cellsig.2011.05.004.

Hess, Katharina; Boger, Christine; Behrens, Hans-Michael; Rocken, Christoph (2014): Correlation between the expression of integrins in prostate cancer and clinical outcome in 1284 patients. In Annals of diagnostic pathology 18 (6), pp. 343-350. DOI: 10.1016/j.anndiagpath.2014.09.001.

Hillisch, Alexander; Peters, Olaf; Kosemund, Dirk; Muller, Gerd; Walter, Alexander; Schneider, Birgitt et al. (2004): Dissecting physiological roles of estrogen receptor alpha and beta with potent selective ligands from structure-based design. In Molecular endocrinology (Baltimore, Md.) 18 (7), pp. 1599-1609. DOI: 10.1210/me.2004-0050.

Holohan Caitriona; van Schaeybroeck, Sandra; Longley, Daniel B.; Johnston, Patrick G. (2013): Cancer drug resistance: an evolving paradigm. In Nature reviews. Cancer 13 (10), pp. 714-726. DOI: 10.1038/nrc3599.

Holzbecher, M. D.; Moss, M. A.; Ellenberger, H. A. (1984): The cyanide content of laetrile preparations, apricot, peach and apple seeds. In Journal of toxicology. Clinical toxicology 22 (4), pp. 341-347.

Holzbeierlein, Jeff; Lal, Priti; LaTulippe, Eva; Smith, Alex; Satagopan, Jaya; Zhang, Liying et al. (2004): Gene Expression Analysis of Human Prostate Carcinoma during Hormonal Therapy Identifies Androgen-Responsive Genes and Mechanisms of Therapy 
Resistance. In The American Journal of Pathology 164 (1), pp. 217-227. DOI: 10.1016/S0002-9440(10)63112-4.

Hornsby, Peter J. (2007): Telomerase and the aging process. In Experimental gerontology 42 (7), pp. 575-581. DOI: 10.1016/j.exger.2007.03.007.

Horvath, L. G.; Henshall, S. M.; Lee, C. S.; Head, D. R.; Quinn, D. I.; Makela, S. et al. (2001): Frequent loss of estrogen receptor-beta expression in prostate cancer. In Cancer research 61 (14), pp. 5331-5335.

Hu, Zhongyi; Gu, Yuanyuan; Han, Bo; Zhang, Jinsan; Li, Zunling; Tian, Keli et al. (2012): Knockdown of AGR2 induces cellular senescence in prostate cancer cells. In Carcinogenesis 33 (6), pp. 1178-1186. DOI: 10.1093/carcin/bgs141.

Huang, Pengxiang; Chandra, Vikas; Rastinejad, Fraydoon (2010): Structural overview of the nuclear receptor superfamily: insights into physiology and therapeutics. In Annual review of physiology 72, pp. 247-272. DOI: 10.1146/annurev-physiol-021909-135917.

Huang, Xingxu; Tran, Thanh; Zhang, Lingna; Hatcher, Rashieda; Zhang, Pumin (2005): DNA damage-induced mitotic catastrophe is mediated by the Chk1-dependent mitotic exit DNA damage checkpoint. In Proceedings of the National Academy of Sciences of the United States of America 102 (4), pp. 1065-1070. DOI: 10.1073/pnas.0409130102.

Huggins, Charles; Hodges, Clarence V. (2002): Studies on prostatic cancer: I. The effect of castration, of estrogen and of androgen injection on serum phosphatases in metastatic carcinoma of the prostate. 1941. In The Journal of urology 168 (1), pp. 9-12.

Hurtado, Antoni; Pinos, Tomas; Barbosa-Desongles, Anna; Lopez-Aviles, Sandra; Barquinero, Jordi; Petriz, Jordi et al. (2008): Estrogen receptor beta displays cell cycledependent expression and regulates the G1 phase through a non-genomic mechanism in prostate carcinoma cells. In Cellular oncology : the official journal of the International Society for Cellular Oncology 30 (4), pp. 349-365.

Hurwitz, A. A.; Foster, B. A.; Allison, J. P.; Greenberg, N. M.; Kwon, E. D. (2001): The TRAMP mouse as a model for prostate cancer. In Current protocols in immunology Chapter 20, Unit 20.5. DOI: 10.1002/0471142735.im2005s45.

Hussain, Shirin; Lawrence, Mitchell G.; Taylor, Renea A.; Lo, Camden Yeung-Wah; Frydenberg, Mark; Ellem, Stuart J. et al. (2012): Estrogen receptor beta activation impairs prostatic regeneration by inducing apoptosis in murine and human stem/progenitor enriched cell populations. In PloS one 7 (7), e40732. DOI: 10.1371/journal.pone.0040732.

Hwang, Hye-Jeong; Kim, Pil; Kim, Chang-Ju; Lee, Hye-Jung; Shim, Insop; Yin, Chang Shik et al. (2008): Antinociceptive effect of amygdalin isolated from Prunus armeniaca on 
formalin-induced pain in rats. In Biological \& pharmaceutical bulletin 31 (8), pp. 1559 1564.

Jain, Shalini; Chakraborty, Goutam; Raja, Remya; Kale, Smita; Kundu, Gopal C. (2008): Prostaglandin E2 regulates tumor angiogenesis in prostate cancer. In Cancer research 68 (19), pp. 7750-7759. DOI: 10.1158/0008-5472.CAN-07-6689.

Jarrard, D. F.; Blitz, B. F.; Smith, R. C.; Patai, B. L.; Rukstalis, D. B. (1994): Effect of epidermal growth factor on prostate cancer cell line PC3 growth and invasion. In The Prostate 24 (1), pp. 46-53.

Johnson, M. (1996): Neoplastic fever. In Palliative medicine 10 (3), pp. 217-224.

Juengel, Eva; Afschar, Masud; Makarevic, Jasmina; Rutz, Jochen; Tsaur, Igor; Mani, Jens et al. (2016): Amygdalin blocks the in vitro adhesion and invasion of renal cell carcinoma cells by an integrin-dependent mechanism. In International journal of molecular medicine 37 (3), pp. 843-850. DOI: 10.3892/ijmm.2016.2454.

Kaarbo, Mari; Mikkelsen, Oyvind Loveseter; Malerod, Lene; Qu, Su; Lobert, Viola H.; Akgul, Gulcan et al. (2010): PI3K-AKT-mTOR pathway is dominant over androgen receptor signaling in prostate cancer cells. In Cellular oncology : the official journal of the International Society for Cellular Oncology 32 (1-2), pp. 11-27. DOI: 10.3233/CLO2009-0487.

Kaighn, M. E.; Narayan, K. S.; Ohnuki, Y.; Lechner, J. F.; Jones, L. W. (1979): Establishment and characterization of a human prostatic carcinoma cell line (PC-3). In Investigative urology 17 (1), pp. 16-23.

Kampa, Maeilena; Papakonstanti, Evangelia A.; Hatzoglou, Anastassia; Stathopoulos, Efstathios N.; Stournaras, Christos; Castanas, Elias (2002): The human prostate cancer cell line LNCaP bears functional membrane testosterone receptors that increase PSA secretion and modify actin cytoskeleton. In FASEB journal : official publication of the Federation of American Societies for Experimental Biology 16 (11), pp. 1429-1431. DOI: 10.1096/fj.02-0131fje.

Karantanos, T.; Corn, P. G.; Thompson, T. C. (2013): Prostate cancer progression after androgen deprivation therapy: mechanisms of castrate resistance and novel therapeutic approaches. In Oncogene 32 (49), pp.5501-5511. DOI: 10.1038/onc.2013.206.

Keating, Gillian M. (2015): Enzalutamide: a review of its use in chemotherapy-naive metastatic castration-resistant prostate cancer. In Drugs \& aging 32 (3), pp. 243-249. DOI: $10.1007 / s 40266-015-0248-y$. 
Kel, Alexdander; Voss, Nico; Jauregui, Ruy; Kel-Margoulis, Olga; Wingender, Edgar (2006): Beyond microarrays: find key transcription factors controlling signal transduction pathways. In BMC bioinformatics 7 Suppl 2, S13. DOI: 10.1186/1471-2105-7-S2-S13.

Khandekar, J. D.; Edelman, H. (1979): Studies of amygdalin (laetrile) toxicity in rodents. In JAMA 242 (2), pp. 169-171.

Kharaishvili, Gvantsa; Simkova, Dana; Makharoblidze, Eka; Trtkova, Katerina; Kolar, Zdenek; Bouchal, Jan (2011): Wnt signaling in prostate development and carcinogenesis. In Biomedical papers of the Medical Faculty of the University Palacky, Olomouc, Czechoslovakia 155 (1), pp. 11-18.

Kikuno, Nobuyuki; Shiina, Hiroaki; Urakami, Shinji; Kawamoto, Ken; Hirata, Hiroshi; Tanaka, Yuichiro et al. (2008): Genistein mediated histone acetylation and demethylation activates tumor suppressor genes in prostate cancer cells. In International journal of cancer 123 (3), pp. 552-560. DOI: 10.1002/ijc.23590.

Kim, H. G.; Kassis, J.; Souto, J. C.; Turner, T.; Wells, A. (1999): EGF receptor signaling in prostate morphogenesis and tumorigenesis. In Histology and histopathology 14 (4), pp. 1175-1182.

Klotz, L.; McNeill, I.; Fleshner, N. (1999): A phase 1-2 trial of diethylstilbestrol plus low dose warfarin in advanced prostate carcinoma. In The Journal of urology 161 (1), pp. 169172.

Kluetz, Paul G.; Figg, William D.; Dahut, William L. (2010): Angiogenesis inhibitors in the treatment of prostate cancer. In Expert opinion on pharmacotherapy 11 (2), pp. 233247. DOI: $10.1517 / 14656560903451716$.

Knudsen, Karen E.; Penning, Trevor M. (2010): Partners in crime: deregulation of AR activity and androgen synthesis in prostate cancer. In Trends in endocrinology and metabolism: TEM 21 (5), pp. 315-324. DOI: 10.1016/j.tem.2010.01.002.

Kong, E. H.; Pike, A. C. W.; Hubbard, R. E. (2003): Structure and mechanism of the oestrogen receptor. In Biochemical Society transactions 31 (Pt 1), pp. 56-59.

Koreckij, Theodore D.; Trauger, Richard J.; Montgomery, Robert Bruce; Pitts, Tiffany E. M.; Coleman, Ilsa; Nguyen, Holly et al. (2009): HE3235 inhibits growth of castrationresistant prostate cancer. In Neoplasia (New York, N.Y.) 11 (11), pp. 1216-1225.

Korpal, Manav; Korn, Joshua M.; Gao, Xueliang; Rakiec, Daniel P.; Ruddy, David A.; Doshi, Shivang et al. (2013): An F876L mutation in androgen receptor confers genetic and phenotypic resistance to MDV3100 (enzalutamide). In Cancer discovery 3 (9), pp. 1030-1043. DOI: 10.1158/2159-8290.CD-13-0142. 
Krege, J. H.; Hodgin, J. B.; Couse, J. F.; Enmark, E.; Warner, M.; Mahler, J. F. et al. (1998): Generation and reproductive phenotypes of mice lacking estrogen receptor beta. In Proceedings of the National Academy of Sciences of the United States of America 95 (26), pp. 15677-15682.

Kroemer, G.; Dallaporta, B.; Resche-Rigon, M. (1998): The mitochondrial death/life regulator in apoptosis and necrosis. In Annual review of physiology 60, pp. 619-642. DOI: 10.1146/annurev.physiol.60.1.619.

Krycer, James Robert; Brown, Andrew John (2013): Cholesterol accumulation in prostate cancer: a classic observation from a modern perspective. In Biochimica et biophysica acta 1835 (2), pp. 219-229. DOI: 10.1016/j.bbcan.2013.01.002.

Kulski, Jerzy K. (Ed.) (2016): Next Generation Sequencing - Advances, Applications and Challenges: InTech.

Kumi-Diaka, James; Sanderson, Naeyhi-Aelei; Hall, Alexis (2000): The mediating role of caspase-3 protease in the intracellular mechanism of genistein-induced apoptosis in human prostatic carcinoma cell lines, DU145 and LNCaP. In Biology of the Cell 92 (89), pp. 595-604. DOI: 10.1016/S0248-4900(00)01109-6.

Kypta, Robert M.; Waxman, Jonathan (2012): Wnt/beta-catenin signalling in prostate cancer. In Nature reviews. Urology 9 (8), pp. 418-428. DOI: 10.1038/nrurol.2012.116.

Lazennec, Gwendal (2006): Estrogen receptor beta, a possible tumor suppressor involved in ovarian carcinogenesis. In Cancer letters 231 (2), pp. 151-157. DOI: 10.1016/j.canlet.2005.01.021.

Leav, I.; Lau, K. M.; Adams, J. Y.; McNeal, J. E.; Taplin, M. E.; Wang, J. et al. (2001): Comparative studies of the estrogen receptors beta and alpha and the androgen receptor in normal human prostate glands, dysplasia, and in primary and metastatic carcinoma. In The American Journal of Pathology 159 (1), pp. 79-92.

Lee, Bo Yun; Han, Jung A.; Im, Jun Sub; Morrone, Amelia; Johung, Kimberly; Goodwin, Edward C. et al. (2006): Senescence-associated beta-galactosidase is lysosomal betagalactosidase. In Aging cell 5 (2), pp. 187-195. DOI: 10.1111/j.14749726.2006.00199.x.

Lee, Hye-Rim; Kim, Tae-Hee; Choi, Kyung-Chul (2012): Functions and physiological roles of two types of estrogen receptors, ERalpha and ERbeta, identified by estrogen receptor knockout mouse. In Laboratory animal research 28 (2), pp.71-76. DOI: 10.5625/lar.2012.28.2.71.

Lee, Seung Ho; Hatakeyama, Shingo; Yu, Shin-Yi; Bao, Xingfeng; Ohyama, Chikara; Khoo, Kai-Hooi et al. (2009): Core3 O-glycan synthase suppresses tumor formation and 
metastasis of prostate carcinoma PC3 and LNCaP cells through down-regulation of alpha2beta1 integrin complex. In The Journal of biological chemistry 284 (25), pp. 17157-17169. DOI: 10.1074/jbc.M109.010934.

Leotoing, Laurent; Manin, Michele; Monte, Didier; Baron, Silvere; Communal, Yves; Lours, Corinne et al. (2007): Crosstalk between androgen receptor and epidermal growth factor receptor-signalling pathways: a molecular switch for epithelial cell differentiation. In Journal of molecular endocrinology 39 (2), pp. 151-162. DOI: 10.1677/JME-070021.

Leung, Yuet-Kin; Lam, Hung-Ming; Wu, Shulin; Song, Dan; Levin, Linda; Cheng, Liang et al. (2010): Estrogen receptor beta2 and beta5 are associated with poor prognosis in prostate cancer, and promote cancer cell migration and invasion. In Endocrine-related cancer 17 (3), pp. 675-689. DOI: 10.1677/ERC-09-0294.

Levine, Arnold J.; Oren, Moshe (2009): The first 30 years of p53: growing ever more complex. In Nature reviews. Cancer 9 (10), pp. 749-758. DOI: 10.1038/nrc2723.

Li, L.; Prabhakaran, K.; Mills, E. M.; Borowitz, J. L.; Isom, G. E. (2005): Enhancement of cyanide-induced mitochondrial dysfunction and cortical cell necrosis by uncoupling protein-2. In Toxicological sciences : an official journal of the Society of Toxicology 86 (1), pp. 116-124. DOI: 10.1093/toxsci/kfi164.

Li, Xiaodong; Huang, Jing; Yi, Ping; Bambara, Robert A.; Hilf, Russell; Muyan, Mesut (2004): Single-chain estrogen receptors (ERs) reveal that the ERalpha/beta heterodimer emulates functions of the ERalpha dimer in genomic estrogen signaling pathways. In Molecular and cellular biology 24 (17), pp. 7681-7694. DOI: 10.1128/MCB.24.17.76817694.2004.

Liao, Ross S.; Ma, Shihong; Miao, Lu; Li, Rui; Yin, Yi; Raj, Ganesh V. (2013): Androgen receptor-mediated non-genomic regulation of prostate cancer cell proliferation. In Translational andrology and urology 2 (3), pp. 187-196. DOI: 10.3978/j.issn.22234683.2013.09.07.

Lindberg, Marie K.; Moverare, Sofia; Skrtic, Stanko; Gao, Hui; Dahlman-Wright, Karin; Gustafsson, Jan-Ake; Ohlsson, Claes (2003): Estrogen receptor (ER)-beta reduces ERalpha-regulated gene transcription, supporting a "ying yang" relationship between ERalpha and ERbeta in mice. In Molecular endocrinology (Baltimore, Md.) 17 (2), pp. 203-208. DOI: 10.1210/me.2002-0206.

Linja, M. J.; Savinainen, K. J.; Saramaki, O. R.; Tammela, T. L.; Vessella, R. L.; Visakorpi, T. (2001): Amplification and overexpression of androgen receptor gene in hormonerefractory prostate cancer. In Cancer research 61 (9), pp. 3550-3555. 
Liu, Pixu; Cheng, Hailing; Roberts, Thomas M.; Zhao, Jean J. (2009): Targeting the phosphoinositide 3-kinase pathway in cancer. In Nature reviews. Drug discovery 8 (8), pp. 627-644. DOI: 10.1038/nrd2926.

Lonergan, Peter E.; Tindall, Donald J. (2011): Androgen receptor signaling in prostate cancer development and progression. In Journal of carcinogenesis 10, p. 20. DOI: 10.4103/1477-3163.83937.

Loriot, Y.; Bianchini, D.; Ileana, E.; Sandhu, S.; Patrikidou, A.; Pezaro, C. et al. (2013): Antitumour activity of abiraterone acetate against metastatic castration-resistant prostate cancer progressing after docetaxel and enzalutamide (MDV3100). In Annals of oncology : official journal of the European Society for Medical Oncology / ESMO 24 (7), pp. 1807-1812. DOI: 10.1093/annonc/mdt136.

Lu, B.; Leygue, E.; Dotzlaw, H.; Murphy, L. J.; Murphy, L. C.; Watson, P. H. (1998): Estrogen receptor-beta mRNA variants in human and murine tissues. In Molecular and cellular endocrinology 138 (1-2), pp. 199-203.

Lubahn, D. B.; Moyer, J. S.; Golding, T. S.; Couse, J. F.; Korach, K. S.; Smithies, O. (1993): Alteration of reproductive function but not prenatal sexual development after insertional disruption of the mouse estrogen receptor gene. In Proceedings of the National Academy of Sciences of the United States of America 90 (23), pp. 11162-11166.

Lund, Trent D.; Munson, Daniel J.; Adlercreutz, Herman; Handa, Robert J.; Lephart, Edwin D. (2004): Androgen receptor expression in the rat prostate is down-regulated by dietary phytoestrogens. In Reproductive biology and endocrinology : RB\&E 2, p. 5. DOI: $10.1186 / 1477-7827-2-5$.

Maggiolini, Marcello; Recchia, Anna Grazia; Carpino, Amalia; Vivacqua, Adele; Fasanella, Giovanna; Rago, Vittoria et al. (2004): Oestrogen receptor beta is required for androgen-stimulated proliferation of LNCaP prostate cancer cells. In Journal of molecular endocrinology 32 (3), pp. 777-791.

Makarevic, Jasmina; Rutz, Jochen; Juengel, Eva; Kaulfuss, Silke; Reiter, Michael; Tsaur, Igor et al. (2014): Amygdalin blocks bladder cancer cell growth in vitro by diminishing cyclin A and cdk2. In PloS one 9 (8), e105590. DOI: 10.1371/journal.pone.0105590.

Makarevic, Jasmina; Tsaur, Igor; Juengel, Eva; Borgmann, Hendrik; Nelson, Karen; Thomas, Christian et al. (2016): Amygdalin delays cell cycle progression and blocks growth of prostate cancer cells in vitro. In Life sciences 147, pp. 137-142. DOI: 10.1016/j.lfs.2016.01.039.

Malamas, Michael S.; Manas, Eric S.; McDevitt, Robert E.; Gunawan, Iwan; Xu, Zhang B.; Collini, Michael D. et al. (2004): Design and synthesis of aryl diphenolic azoles as 
potent and selective estrogen receptor-beta ligands. In Journal of medicinal chemistry 47 (21), pp. 5021-5040. DOI: 10.1021/jm049719y.

Maneix, Laure; Antonson, Per; Humire, Patricia; Rochel-Maia, Sabrina; Castaneda, Jessica; Omoto, Yoko et al. (2015): Estrogen receptor beta exon 3-deleted mouse: The importance of non-ERE pathways in ERbeta signaling. In Proceedings of the National Academy of Sciences of the United States of America 112 (16), pp. 5135-5140. DOI: 10.1073/pnas. 1504944112.

Mansilla, Sylvia; Priebe, Waldemar; Portugal, Jose (2006): Mitotic catastrophe results in cell death by caspase-dependent and caspase-independent mechanisms. In Cell cycle (Georgetown, Tex.) 5 (1), pp. 53-60. DOI: 10.4161/cc.5.1.2267.

Mantovani, Alberto; Allavena, Paola; Sica, Antonio; Balkwill, Frances (2008): Cancer-related inflammation. In Nature 454 (7203), pp. 436-444. DOI: 10.1038/nature07205.

Massie, Charles E.; Mills, lan G.; Lynch, Andy G. (2016): The importance of DNA methylation in prostate cancer development. In The Journal of steroid biochemistry and molecular biology. DOI: 10.1016/j.jsbmb.2016.04.009.

McPherson, Stephen J.; Ellem, Stuart J.; Simpson, Evan R.; Patchev, Vladimir; Fritzemeier, Karl-Heinrich; Risbridger, Gail P. (2007): Essential role for estrogen receptor beta in stromal-epithelial regulation of prostatic hyperplasia. In Endocrinology 148 (2), pp. 566-574. DOI: 10.1210/en.2006-0906.

McPherson, Stephen J.; Hussain, Shirin; Balanathan, Preetika; Hedwards, Shelley L.; Niranjan, Birunthi; Grant, Michael et al. (2010): Estrogen receptor-beta activated apoptosis in benign hyperplasia and cancer of the prostate is androgen independent and TNFalpha mediated. In Proceedings of the National Academy of Sciences of the United States of America 107 (7), pp. 3123-3128. DOI: 10.1073/pnas.0905524107.

Mentor-Marcel, R.; Lamartiniere, C. A.; Eltoum, I. E.; Greenberg, N. M.; Elgavish, A. (2001): Genistein in the diet reduces the incidence of poorly differentiated prostatic adenocarcinoma in transgenic mice (TRAMP). In Cancer research 61 (18), pp. 67776782.

Michaud, Jason E.; Billups, Kevin L.; Partin, Alan W. (2015): Testosterone and prostate cancer: an evidence-based review of pathogenesis and oncologic risk. In Therapeutic advances in urology 7 (6), pp. 378-387. DOI: 10.1177/1756287215597633.

Milazzo, Stefania; Ernst, Edzard; Lejeune, Stephane; Boehm, Katja; Horneber, Markus (2011): Laetrile treatment for cancer. In The Cochrane database of systematic reviews (11), CD005476. DOI: 10.1002/14651858.CD005476.pub3. 
Minutolo, Filippo; Bertini, Simone; Granchi, Carlotta; Marchitiello, Teresa; Prota, Giovanni; Rapposelli, Simona et al. (2009): Structural evolutions of salicylaldoximes as selective agonists for estrogen receptor beta. In Journal of medicinal chemistry 52 (3), pp. 858867. DOI: 10.1021/jm801458t.

Miyamoto, H.; Yeh, S.; Lardy, H.; Messing, E.; Chang, C. (1998): Delta5-androstenediol is a natural hormone with androgenic activity in human prostate cancer cells. In Proceedings of the National Academy of Sciences of the United States of America 95 (19), pp. 11083-11088.

Mladenov, Emil; lliakis, George (2011): Induction and repair of DNA double strand breaks: the increasing spectrum of non-homologous end joining pathways. In Mutation research 711 (1-2), pp. 61-72. DOI: 10.1016/j.mrfmmm.2011.02.005.

Moertel, C. G.; Ames, M. M.; Kovach, J. S.; Moyer, T. P.; Rubin, J. R.; Tinker, J. H. (1981): A pharmacologic and toxicological study of amygdalin. In JAMA 245 (6), pp. 591-594.

Moertel, C. G.; Fleming, T. R.; Rubin, J.; Kvols, L. K.; Sarna, G.; Koch, R. et al. (1982): A clinical trial of amygdalin (Laetrile) in the treatment of human cancer. In The New England journal of medicine 306 (4), pp. 201-206. DOI: 10.1056/NEJM198201283060403.

Montgomery, R. Bruce; Mostaghel, Elahe A.; Vessella, Robert; Hess, David L.; Kalhorn, Thomas F.; Higano, Celestia S. et al. (2008): Maintenance of intratumoral androgens in metastatic prostate cancer: a mechanism for castration-resistant tumor growth. In Cancer research 68 (11), pp. 4447-4454. DOI: 10.1158/0008-5472.CAN-08-0249.

Moon, Ji-Yoon; Kim, Sang-Won; Yun, Gi-Mok; Lee, Hyeon-Sik; Kim, Yoon-Dong; Jeong, Gie-Joon et al. (2015): Inhibition of cell growth and down-regulation of telomerase activity by amygdalin in human cancer cell lines. In Animal Cells and Systems 19 (5), pp. 295-304. DOI: 10.1080/19768354.2015.1060261.

Morris, Michael J.; Huang, Daisy; Kelly, William K.; Slovin, Susan F.; Stephenson, Ryan D.; Eicher, Caitlin et al. (2009): Phase 1 trial of high-dose exogenous testosterone in patients with castration-resistant metastatic prostate cancer. In European urology 56 (2), pp. 237-244. DOI: 10.1016/j.eururo.2009.03.073.

Morrissey, Colm; Watson, R. William G. (2003): Phytoestrogens and prostate cancer. In Current drug targets 4 (3), pp. 231-241.

Mostaghel, Elahe A. (2013): Steroid hormone synthetic pathways in prostate cancer. In Translational andrology and urology 2 (3), pp. 212-227. DOI: 10.3978/j.issn.22234683.2013.09.16. 
Mostaghel, Elahe A.; Marck, Brett T.; Plymate, Stephen R.; Vessella, Robert L.; Balk, Stephen; Matsumoto, Alvin M. et al. (2011): Resistance to CYP17A1 inhibition with abiraterone in castration-resistant prostate cancer: induction of steroidogenesis and androgen receptor splice variants. In Clinical cancer research : an official journal of the American Association for Cancer Research 17 (18), pp. 5913-5925. DOI: 10.1158/1078-0432.CCR-11-0728.

Muller, Patricia A. J.; Vousden, Karen H.; Norman, Jim C. (2011): p53 and its mutants in tumor cell migration and invasion. In The Journal of cell biology 192 (2), pp. 209-218. DOI: $10.1083 / j c b .201009059$.

Murnane, John P. (2012): Telomere dysfunction and chromosome instability. In Mutation research 730 (1-2), pp. 28-36. DOI: 10.1016/j.mrfmmm.2011.04.008.

Murtola, Teemu J.; Syvala, Heimo; Pennanen, Pasi; Blauer, Merja; Solakivi, Tiina; Ylikomi, Timo; Tammela, Teuvo L. J. (2012): The importance of LDL and cholesterol metabolism for prostate epithelial cell growth. In PloS one 7 (6), e39445. DOI: 10.1371/journal.pone.0039445.

Nakamura, Asako J.; Chiang, Y. Jeffrey; Hathcock, Karen S.; Horikawa, Izumi; Sedelnikova, Olga A.; Hodes, Richard J.; Bonner, William M. (2008): Both telomeric and nontelomeric DNA damage are determinants of mammalian cellular senescence. In Epigenetics \& chromatin 1 (1), p. 6. DOI: 10.1186/1756-8935-1-6.

Nakamura, Yasuhiro; Felizola, Saulo J. A.; Kurotaki, Yumi; Fujishima, Fumiyoshi; McNamara, Keely M.; Suzuki, Takashi et al. (2013): Cyclin D1 (CCND1) expression is involved in estrogen receptor beta (ERbeta) in human prostate cancer. In The Prostate 73 (6), pp. 590-595. DOI: 10.1002/pros.22599.

Newmark, J.; Brady, R. O.; Grimley, P. M.; Gal, A. E.; Waller, S. G.; Thistlethwaite, J. R. (1981): Amygdalin (Laetrile) and prunasin beta-glucosidases: distribution in germ-free rat and in human tumor tissue. In Proceedings of the National Academy of Sciences of the United States of America 78 (10), pp. 6513-6516.

Nikoletopoulou, Vassiliki; Markaki, Maria; Palikaras, Konstantinos; Tavernarakis, Nektarios (2013): Crosstalk between apoptosis, necrosis and autophagy. In Biochimica et biophysica acta 1833 (12), pp. 3448-3459. DOI: 10.1016/j.bbamcr.2013.06.001.

Nupponen, N. N.; Kakkola, L.; Koivisto, P.; Visakorpi, T. (1998): Genetic alterations in hormone-refractory recurrent prostate carcinomas. In The American Journal of Pathology 153 (1), pp. 141-148. DOI: 10.1016/S0002-9440(10)65554-X. 
Odoul, M.; Fouillet, B.; Nouri, B.; Chambon, R.; Chambon, P. (1994): Specific determination of cyanide in blood by headspace gas chromatography. In Journal of analytical toxicology 18 (4), pp. 205-207.

Olsen, Jan Roger; Azeem, Waqas; Hellem, Margrete Reime; Marvyin, Kristo; Hua, Yaping; $\mathrm{Qu}, \mathrm{Yi}$ et al. (2016): Context dependent regulatory patterns of the androgen receptor and androgen receptor target genes. In BMC cancer 16, p. 377. DOI: 10.1186/s12885016-2453-4.

Palmieri, C.; Cheng, G. J.; Saji, S.; Zelada-Hedman, M.; Warri, A.; Weihua, Z. et al. (2002): Estrogen receptor beta in breast cancer. In Endocrine-related cancer 9 (1), pp. 1-13.

Papakonstanti, Evangelia A.; Kampa, Marilena; Castanas, Elias; Stournaras, Christos (2003): A rapid, nongenomic, signaling pathway regulates the actin reorganization induced by activation of membrane testosterone receptors. In Molecular endocrinology (Baltimore, Md.) 17 (5), pp. 870-881. DOI: 10.1210/me.2002-0253.

Parrinello, Simona; Samper, Enrique; Krtolica, Ana; Goldstein, Joshua; Melov, Simon; Campisi, Judith (2003): Oxygen sensitivity severely limits the replicative lifespan of murine fibroblasts. In Nature cell biology 5 (8), pp. 741-747. DOI: 10.1038/ncb1024.

Paul, R.; Breul, J. (2000): Antiandrogen withdrawal syndrome associated with prostate cancer therapies: incidence and clinical significance. In Drug safety 23 (5), pp. 381390.

Peekhaus, N. T.; Chang, T.; Hayes, E. C.; Wilkinson, H. A.; Mitra, S. W.; Schaeffer, J. M.; Rohrer, S. P. (2004): Distinct effects of the antiestrogen Faslodex on the stability of estrogen receptors-alpha and -beta in the breast cancer cell line MCF-7. In Journal of molecular endocrinology 32 (3), pp. 987-995.

Pelton, Kristine; Freeman, Michael R.; Solomon, Keith R. (2012): Cholesterol and prostate cancer. In Current opinion in pharmacology 12 (6), pp.751-759. DOI: 10.1016/j.coph.2012.07.006.

Perner, Sven; Rupp, Niels J.; Braun, Martin; Rubin, Mark A.; Moch, Holger; Dietel, Manfred et al. (2013): Loss of SLC45A3 protein (prostein) expression in prostate cancer is associated with SLC45A3-ERG gene rearrangement and an unfavorable clinical course. In International journal of cancer 132 (4), pp. 807-812. DOI: 10.1002/ijc.27733.

Pettersen, John C.; Cohen, Steven D. (1993): The effects of cyanide on brain mitochondrial cytochrome oxidase and respiratory activities. In J. Appl. Toxicol. 13 (1), pp. 9-14. DOI: 10.1002/jat.2550130104.

Polager, Shirley; Ginsberg, Doron (2009): p53 and E2f: partners in life and death. In Nature reviews. Cancer 9 (10), pp. 738-748. DOI: 10.1038/nrc2718. 
Prabhakaran, K.; Li, L.; Borowitz, J. L.; Isom, G. E. (2002): Cyanide induces different modes of death in cortical and mesencephalon cells. In The Journal of pharmacology and experimental therapeutics 303 (2), pp. 510-519. DOI: 10.1124/jpet.102.039453.

Prensner, John R.; Chen, Wei; Han, Sumin; Iyer, Matthew K.; Cao, Qi; Kothari, Vishal et al. (2014a): The long non-coding RNA PCAT-1 promotes prostate cancer cell proliferation through cMyc. In Neoplasia (New York, N.Y.) 16 (11), pp. 900-908. DOI: 10.1016/j.neo.2014.09.001.

Prensner, John R.; Chen, Wei; lyer, Matthew K.; Cao, Qi; Ma, Teng; Han, Sumin et al. (2014b): PCAT-1, a long noncoding RNA, regulates BRCA2 and controls homologous recombination in cancer. In Cancer research 74 (6), pp. 1651-1660. DOI: 10.1158/0008-5472.CAN-13-3159.

Prensner, John R.; Iyer, Matthew K.; Balbin, O. Alejandro; Dhanasekaran, Saravana M.; Cao, Qi; Brenner, J. Chad et al. (2011): Transcriptome sequencing across a prostate cancer cohort identifies PCAT-1, an unannotated lincRNA implicated in disease progression. In Nature biotechnology 29 (8), pp. 742-749. DOI: 10.1038/nbt.1914.

Price, R. H., JR; Butler, C. A.; Webb, P.; Uht, R.; Kushner, P.; Handa, R. J. (2001): A splice variant of estrogen receptor beta missing exon 3 displays altered subnuclear localization and capacity for transcriptional activation. In Endocrinology 142 (5), pp. 2039-2049. DOI: 10.1210/endo.142.5.8130.

Prieur, Alexandre; Peeper, Daniel S. (2008): Cellular senescence in vivo: a barrier to tumorigenesis. In Current opinion in cell biology 20 (2), pp. 150-155. DOI: 10.1016/j.ceb.2008.01.007.

Prins, Gail S.; Korach, Kenneth S. (2008): The role of estrogens and estrogen receptors in normal prostate growth and disease. In Steroids 73 (3), pp. 233-244. DOI: 10.1016/j.steroids.2007.10.013.

Proskuryakov, Sergey Y.; Gabai, Vladimir L. (2010): Mechanisms of tumor cell necrosis. In Current pharmaceutical design 16 (1), pp. 56-68.

Proskuryakov, Sergey Y.a; Konoplyannikov, Anatoli G.; Gabai, Vladimir L. (2003): Necrosis. A specific form of programmed cell death? In Experimental cell research 283 (1), pp. 1-16. DOI: 10.1016/S0014-4827(02)00027-7.

Qi, Wenqing; Morales, Carla; Cooke, Laurence S.; Johnson, Benny; Somer, Bradley; Mahadevan, Daruka (2015): Reciprocal feedback inhibition of the androgen receptor and PI3K as a novel therapy for castrate-sensitive and -resistant prostate cancer. In Oncotarget 6 (39), pp. 41976-41987. DOI: 10.18632/oncotarget.5659. 
Qian, Liyu; Xie, Bo; Wang, Yaguo; Qian, Jun (2015): Amygdalin-mediated inhibition of nonsmall cell lung cancer cell invasion in vitro. In International journal of clinical and experimental pathology 8 (5), pp. 5363-5370.

Qian, Yingjuan; Chen, Xinbin (2013): Senescence regulation by the p53 protein family. In Methods in molecular biology (Clifton, N.J.) 965, pp. 37-61. DOI: 10.1007/978-162703-239-1_3.

Quarmby, V. E.; Yarbrough, W. G.; Lubahn, D. B.; French, F. S.; Wilson, E. M. (1990): Autologous down-regulation of androgen receptor messenger ribonucleic acid. In Molecular endocrinology (Baltimore, Md.) 4 (1), pp. 22-28. DOI: 10.1210/mend-4-1-22.

Rauws, A. G.; Olling, M.; Timmerman, A. (1982): The pharmacokinetics of amygdalin. In Archives of toxicology 49 (3-4), pp. 311-319.

Ravnskov, U. (2002): Is atherosclerosis caused by high cholesterol? In QJM 95 (6), pp. 397-403. DOI: 10.1093/qjmed/95.6.397.

Ricke, William A.; McPherson, Stephen J.; Bianco, Joseph J.; Cunha, Gerald R.; Wang, Yuzhuo; Risbridger, Gail P. (2008): Prostatic hormonal carcinogenesis is mediated by in situ estrogen production and estrogen receptor alpha signaling. In FASEB journal : official publication of the Federation of American Societies for Experimental Biology 22 (5), pp. 1512-1520. DOI: 10.1096/fj.07-9526com.

Robert-Koch-Institut; Gesellschaft der epidemiologischen Krebsregister in Deutschland (2015): Krebs in Deutschland 2011/2012. 10. Ausg. Berlin (Beiträge zur Gesundheitsberichterstattung des Bundes).

Robles, S. J.; Adami, G. R. (1998): Agents that cause DNA double strand breaks lead to p16INK4a enrichment and the premature senescence of normal fibroblasts. In Oncogene 16 (9), pp. 1113-1123. DOI: 10.1038/sj.onc.1201862.

Rock, Kenneth L.; Kono, Hajime (2008): The inflammatory response to cell death. In Annual review of pathology 3, pp.99-126. DOI: 10.1146/annurev.pathmechdis.3.121806.151456.

Rodier, Francis; Kim, Sahn-Ho; Nijjar, Tarlochan; Yaswen, Paul; Campisi, Judith (2005): Cancer and aging: the importance of telomeres in genome maintenance. In The international journal of biochemistry \& cell biology 37 (5), pp. 977-990. DOI: 10.1016/j.biocel.2004.10.012.

Rodriguez-Vida, Alejo; Galazi, Myria; Rudman, Sarah; Chowdhury, Simon; Sternberg, Cora N. (2015): Enzalutamide for the treatment of metastatic castration-resistant prostate cancer. In Drug design, development and therapy 9, pp.3325-3339. DOI: 10.2147/DDDT.S69433. 
Roitelman, J.; Simoni, R. D. (1992): Distinct sterol and nonsterol signals for the regulated degradation of 3-hydroxy-3-methylglutaryl-CoA reductase. In The Journal of biological chemistry 267 (35), pp. 25264-25273.

Roninson, Igor B. (2003): Tumor cell senescence in cancer treatment. In Cancer research 63 (11), pp. 2705-2715.

Royuela, M.; Miguel, M. P. de; Bethencourt, F. R.; Sanchez-Chapado, M.; Fraile, B.; Arenas, M. I.; Paniagua, R. (2001): Estrogen receptors alpha and beta in the normal, hyperplastic and carcinomatous human prostate. In The Journal of endocrinology 168 (3), pp. 447-454.

Saiki, R. K.; Scharf, S.; Faloona, F.; Mullis, K. B.; Horn, G. T.; Erlich, H. A.; Arnheim, N. (1985): Enzymatic amplification of beta-globin genomic sequences and restriction site analysis for diagnosis of sickle cell anemia. In Science (New York, N.Y.) 230 (4732), pp. 1350-1354.

Sanger, F.; Nicklen, S.; Coulson, A. R. (1977): DNA sequencing with chain-terminating inhibitors. In Proceedings of the National Academy of Sciences of the United States of America 74 (12), pp. 5463-5467.

Sartor, A. Oliver; Tangen, Catherine M.; Hussain, Maha H. A.; Eisenberger, Mario A.; Parab, Minoti; Fontana, Joseph A. et al. (2008): Antiandrogen withdrawal in castrate-refractory prostate cancer: a Southwest Oncology Group trial (SWOG 9426). In Cancer 112 (11), pp. 2393-2400. DOI: 10.1002/cncr.23473.

Scherer G. et al. (2017) Thiocyanat. The MAK Collection for Occupational Health and Safety, under Revision

Schrader, Andres Jan; Boegemann, Martin; Ohlmann, Carsten-H; Schnoeller, Thomas J.; Krabbe, Laura-Maria; Hajili, Turkan et al. (2014): Enzalutamide in castration-resistant prostate cancer patients progressing after docetaxel and abiraterone. In European urology 65 (1), pp. 30-36. DOI: 10.1016/j.eururo.2013.06.042.

Schwarze, Steven R.; Fu, Vivian X.; Desotelle, Joshua A.; Kenowski, Michelle L.; Jarrard, David F. (2005): The identification of senescence-specific genes during the induction of senescence in prostate cancer cells. In Neoplasia (New York, N.Y.) 7 (9), pp. 816-823.

Seluanov, A.; Gorbunova, V.; Falcovitz, A.; Sigal, A.; Milyavsky, M.; Zurer, I. et al. (2001): Change of the death pathway in senescent human fibroblasts in response to DNA damage is caused by an inability to stabilize p53. In Molecular and cellular biology 21 (5), pp. 1552-1564. DOI: 10.1128/MCB.21.5.1552-1564.2001.

Shan, L. X.; Rodriguez, M. C.; Janne, O. A. (1990): Regulation of androgen receptor protein and mRNA concentrations by androgens in rat ventral prostate and seminal vesicles 
and in human hepatoma cells. In Molecular endocrinology (Baltimore, Md.) 4 (11), pp. 1636-1646. DOI: 10.1210/mend-4-11-1636.

Sharad, S.; Srivastava, A.; Ravulapalli, S.; Parker, P.; Chen, Y.; Li, H. et al. (2011): Prostate cancer gene expression signature of patients with high body mass index. In Prostate cancer and prostatic diseases 14 (1), pp. 22-29. DOI: 10.1038/pcan.2010.44.

Shen, J. C.; Klein, R. D.; Wei, Q.; Guan, Y.; Contois, J. H.; Wang, T. T. et al. (2000): Lowdose genistein induces cyclin-dependent kinase inhibitors and $\mathrm{G}(1)$ cell-cycle arrest in human prostate cancer cells. In Molecular carcinogenesis 29 (2), pp. 92-102.

Shukla, Sanjeev; Maclennan, Gregory T.; Hartman, Douglas J.; Fu, Pingfu; Resnick, Martin I.; Gupta, Sanjay (2007): Activation of PI3K-Akt signaling pathway promotes prostate cancer cell invasion. In International journal of cancer 121 (7), pp. 1424-1432. DOI: 10.1002/ijc.22862.

Singh, Mandeep; Jha, Ruchi; Melamed, Jonathan; Shapiro, Ellen; Hayward, Simon W.; Lee, Peng (2014): Stromal androgen receptor in prostate development and cancer. In The American Journal of Pathology 184 (10), pp. 2598-2607. DOI: 10.1016/j.ajpath.2014.06.022.

Singh, Richa; George, Jasmine; Shukla, Yogeshwer (2010): Role of senescence and mitotic catastrophe in cancer therapy. In Cell division 5, p. 4. DOI: 10.1186/1747-1028-5-4.

Skafar, Debra F.; Zhao, Changqing (2008): The multifunctional estrogen receptor-alpha F domain. In Endocrine 33 (1), pp. 1-8. DOI: 10.1007/s12020-008-9054-1.

Skliris, G. P.; Parkes, A. T.; Limer, J. L.; Burdall, S. E.; Carder, P. J.; Speirs, V. (2002): Evaluation of seven oestrogen receptor beta antibodies for immunohistochemistry, western blotting, and flow cytometry in human breast tissue. In The Journal of pathology 197 (2), pp. 155-162. DOI: 10.1002/path.1077.

Slusarz, Anna; Jackson, Glenn A.; Day, J. Kevin; Shenouda, Nader S.; Bogener, Jennifer L.; Browning, Jim D. et al. (2012): Aggressive prostate cancer is prevented in ERalphaKO mice and stimulated in ERbetaKO TRAMP mice. In Endocrinology 153 (9), pp. 4160 4170. DOI: 10.1210/en.2012-1030.

Smart, Emily; Hughes, Thomas; Smith, Laura; Speirs, Valerie (2013): Estrogen receptor beta: putting a positive into triple negative breast cancer? In Hormone molecular biology and clinical investigation 16 (3), pp. 117-123. DOI: 10.1515/hmbci-2013-0042.

Song, Zuoqing; Xu, Xiaohong (2014): Advanced research on anti-tumor effects of amygdalin. In Journal of cancer research and therapeutics 10 Suppl 1, pp.3-7. DOI: 10.4103/0973-1482.139743. 
Sonn, G. A.; Aronson, W.; Litwin, M. S. (2005): Impact of diet on prostate cancer: a review. In Prostate cancer and prostatic diseases 8 (4), pp. 304-310. DOI: 10.1038/sj.pcan.4500825.

Sotoca M., Ana; Vervoort, Jacques; M.C.M., Ivonne; Gustafsso, Jan-ke (2012): Human ERa and ER $\beta$ Splice Variants: Understanding Their Domain Structure in Relation to Their Biological Roles in Breast Cancer Cell Proliferation. In Deniz Ekinci (Ed.): Biochemistry: InTech.

Srivastava, Sanjeev K.; Bhardwaj, Arun; Singh, Seema; Arora, Sumit; McClellan, Steven; Grizzle, William E. et al. (2012): Myb overexpression overrides androgen depletioninduced cell cycle arrest and apoptosis in prostate cancer cells, and confers aggressive malignant traits: potential role in castration resistance. In Carcinogenesis 33 (6), pp. 1149-1157. DOI: 10.1093/carcin/bgs134.

Stanbrough, M.; Leav, I.; Kwan, P. W.; Bubley, G. J.; Balk, S. P. (2001): Prostatic intraepithelial neoplasia in mice expressing an androgen receptor transgene in prostate epithelium. In Proceedings of the National Academy of Sciences of the United States of America 98 (19), pp. 10823-10828. DOI: 10.1073/pnas.191235898.

Statkiewicz, Malgorzata; Maryan, Natalia; Lipiec, Agnieszka; Grecka, Emilia; Grygorowicz, Monika A.; Omiotek, Marcin et al. (2014): The role of the SHH gene in prostate cancer cell resistance to paclitaxel. In The Prostate 74 (11), pp. 1142-1152. DOI: 10.1002/pros.22830

Stettner, Mark; Kaulfuss, Silke; Burfeind, Peter; Schweyer, Stefan; Strauss, Arne; Ringert, Rolf-Hermann; Thelen, Paul (2007): The relevance of estrogen receptor-beta expression to the antiproliferative effects observed with histone deacetylase inhibitors and phytoestrogens in prostate cancer treatment. In Molecular cancer therapeutics 6 (10), pp. 2626-2633. DOI: 10.1158/1535-7163.MCT-07-0197.

Su, Z.; Yang, Z.; Xie, L.; DeWitt, J. P.; Chen, Y. (2016): Cancer therapy in the necroptosis era. In Cell death and differentiation 23 (5), pp. 748-756. DOI: 10.1038/cdd.2016.8.

Susa, Takao; Ikaga, Reina; Kajitani, Takashi; lizuka, Masayoshi; Okinaga, Hiroko; Tamamori-Adachi, Mimi; Okazaki, Tomoki (2015): Wild-type and specific mutant androgen receptor mediates transcription via 17beta-estradiol in sex hormone-sensitive cancer cells. In Journal of cellular physiology 230 (7), pp. 1594-1606. DOI: 10.1002/jcp.24906.

Swyer, G. I. M. The cholesterol content of normal and enlarged prostates. Cancer Res., 2, 372-375, 1942. 
Tamura, Takahiko; Chiba, Joe (2009): STEAP4 regulates focal adhesion kinase activation and CpG motifs within STEAP4 promoter region are frequently methylated in DU145, human androgen-independent prostate cancer cells. In International journal of molecular medicine 24 (5), pp. 599-604.

Tang, K. D.; Ling, Ming-Tat (2014): Targeting drug-resistant prostate cancer with dual $\mathrm{PI3K} / \mathrm{mTOR}$ inhibition. In Current medicinal chemistry 21 (26), pp. 3048-3056.

Tani, Y.; Suttie, A.; Flake, G. P.; Nyska, A.; Maronpot, R. R. (2005): Epithelial-stromal tumor of the seminal vesicles in the transgenic adenocarcinoma mouse prostate model. In Veterinary pathology 42 (3), pp. 306-314. DOI: 10.1354/vp.42-3-306.

Tarjan, M.; Ottlecz, I.; Tot, T. (2009): Primary adenocarcinoma of the seminal vesicle. In Indian journal of urology : IJU : journal of the Urological Society of India 25 (1), pp. 143-145. DOI: 10.4103/0970-1591.45557.

te Poele, Robert H.; Okorokov, Andrei L.; Jardine, Lesley; Cummings, Jeffrey; Joel, Simon P. (2002): DNA damage is able to induce senescence in tumor cells in vitro and in vivo. In Cancer research 62 (6), pp. 1876-1883.

Teply, B. A.; Luber, B.; Denmeade, S. R.; Antonarakis, E. S. (2016): The influence of prednisone on the efficacy of docetaxel in men with metastatic castration-resistant prostate cancer. In Prostate cancer and prostatic diseases 19 (1), pp. 72-78. DOI: 10.1038/pcan.2015.53.

Thelen, Paul; Heinrich, Elmar; Bremmer, Felix; Trojan, Lutz; Strauss, Arne (2013): Testosterone boosts for treatment of castration resistant prostate cancer: an experimental implementation of intermittent androgen deprivation. In The Prostate 73 (15), pp. 1699-1709. DOI: 10.1002/pros.22711.

Thelen, Paul; Peter, Thomas; Hunermund, Anika; Kaulfuss, Silke; Seidlova-Wuttke, Dana; Wuttke, Wolfgang et al. (2007): Phytoestrogens from Belamcanda chinensis regulate the expression of steroid receptors and related cofactors in LNCaP prostate cancer cells. In BJU international 100 (1), pp. 199-203. DOI: 10.1111/j.1464410X.2007.06924.x.

Tominaga, S. (1985): Cancer incidence in Japanese in Japan, Hawaii, and western United States. In National Cancer Institute monograph 69, pp. 83-92.

Trachtenberg, J.; Bogaards, A.; Weersink, R. A.; Haider, M. A.; Evans, A.; McCluskey, S. A. et al. (2007): Vascular targeted photodynamic therapy with palladiumbacteriopheophorbide photosensitizer for recurrent prostate cancer following definitive radiation therapy: assessment of safety and treatment response. In The Journal of urology 178 (5), 1974-9; discussion 1979. DOI: 10.1016/j.juro.2007.07.036. 
Tran, Chris; Ouk, Samedy; Clegg, Nicola J.; Chen, Yu; Watson, Philip A.; Arora, Vivek et al. (2009): Development of a second-generation antiandrogen for treatment of advanced prostate cancer. In Science (New York, N.Y.) 324 (5928), pp. 787-790. DOI: 10.1126/science.1168175.

Trauger, R.; Corey, E.; Bell, D.; White, S.; Garsd, A.; Stickney, D. et al. (2009): Inhibition of androstenediol-dependent LNCaP tumour growth by 17alpha-ethynyl-5alphaandrostane-3alpha, 17beta-diol (HE3235). In British journal of cancer 100 (7), pp. 1068-1072. DOI: 10.1038/sj.bjc.6604987.

Tselis, Nikolaos; Tunn, Ulf W.; Chatzikonstantinou, Georgios; Milickovic, Natasa; Baltas, Dimos; Ratka, Markus; Zamboglou, Nikolaos (2013): High dose rate brachytherapy as monotherapy for localised prostate cancer: a hypofractionated two-implant approach in 351 consecutive patients. In Radiation oncology (London, England) 8, p. 115. DOI: 10.1186/1748-717X-8-115.

Tu, Ho-Chou; Ren, Decheng; Wang, Gary X.; Chen, David Y.; Westergard, Todd D.; Kim, Hyungjin et al. (2009): The p53-cathepsin axis cooperates with ROS to activate programmed necrotic death upon DNA damage. In Proceedings of the National Academy of Sciences of the United States of America 106 (4), pp. 1093-1098. DOI: 10.1073/pnas.0808173106.

Turner, Joseph V.; Agatonovic-Kustrin, Snezana; Glass, Beverley D. (2007): Molecular aspects of phytoestrogen selective binding at estrogen receptors. In Journal of pharmaceutical sciences 96 (8), pp. 1879-1885. DOI: 10.1002/jps.20987.

Unproven methods of cancer management. Laetrile (1991). In CA: a cancer journal for clinicians 41 (3), pp. 187-192.

Vakifahmetoglu, H.; Olsson, M.; Zhivotovsky, B. (2008): Death through a tragedy: mitotic catastrophe. In Cell death and differentiation 15 (7), pp. 1153-1162. DOI: 10.1038/cdd.2008.47.

Vanlangenakker, N.; Vanden Berghe, T.; Vandenabeele, P. (2012): Many stimuli pull the necrotic trigger, an overview. In Cell death and differentiation 19 (1), pp. 75-86. DOI: 10.1038/cdd.2011.164.

Vaseva, Angelina V.; Marchenko, Natalie D.; Ji, Kyungmin; Tsirka, Stella E.; Holzmann, Sonja; Moll, Ute M. (2012): p53 opens the mitochondrial permeability transition pore to trigger necrosis. In Cell 149 (7), pp. 1536-1548. DOI: 10.1016/j.cell.2012.05.014.

Vaz, Catia V.; Alves, Marco G.; Marques, Ricardo; Moreira, Paula I.; Oliveira, Pedro F.; Maia, Claudio J.; Socorro, Silvia (2012): Androgen-responsive and nonresponsive prostate cancer cells present a distinct glycolytic metabolism profile. In The international journal 

of biochemistry \& cell biology 4
10.1016/j.biocel.2012.08.013.
(11), pp. 2077-2084. DOI:

Veierod, M. B.; Laake, P.; Thelle, D. S. (1997): Dietary fat intake and risk of prostate cancer: a prospective study of 25,708 Norwegian men. In International journal of cancer 73 (5), pp. 634-638.

Veldscholte, J.; Berrevoets, C. A.; Ris-Stalpers, C.; Kuiper, G. G.; Jenster, G.; Trapman, J. et al. (1992): The androgen receptor in LNCaP cells contains a mutation in the ligand binding domain which affects steroid binding characteristics and response to antiandrogens. In The Journal of steroid biochemistry and molecular biology 41 (3-8), pp. 665-669.

Visakorpi, T.; Kallioniemi, A. H.; Syvanen, A. C.; Hyytinen, E. R.; Karhu, R.; Tammela, T. et al. (1995): Genetic changes in primary and recurrent prostate cancer by comparative genomic hybridization. In Cancer research 55 (2), pp. 342-347.

Vitale, Ilio; Galluzzi, Lorenzo; Castedo, Maria; Kroemer, Guido (2011): Mitotic catastrophe: a mechanism for avoiding genomic instability. In Nature reviews. Molecular cell biology 12 (6), pp. 385-392. DOI: 10.1038/nrm3115.

Vivar, Omar I.; Zhao, Xiaoyue; Saunier, Elise F.; Griffin, Chandi; Mayba, Oleg S.; Tagliaferri, Mary et al. (2010): Estrogen receptor beta binds to and regulates three distinct classes of target genes. In The Journal of biological chemistry 285 (29), pp. 22059-22066. DOI: $10.1074 / j b c . M 110.114116$.

Vock, E. H.; Lutz, W. K.; Hormes, P.; Hoffmann, H. D.; Vamvakas, S. (1998): Discrimination between genotoxicity and cytotoxicity in the induction of DNA double-strand breaks in cells treated with etoposide, melphalan, cisplatin, potassium cyanide, Triton X-100, and gamma-irradiation. In Mutation research 413 (1), pp. 83-94.

von Hardenberg, S. 2010: Charakterisierung von Leupaxin und seiner Interaktionspartner in Karzinomzellen. Dissertation. University Göttingen, Germany. Institute for Human Genetics

Waltering, Kati K.; Helenius, Merja A.; Sahu, Biswajyoti; Manni, Visa; Linja, Marika J.; Janne, Olli A.; Visakorpi, Tapio (2009): Increased expression of androgen receptor sensitizes prostate cancer cells to low levels of androgens. In Cancer research 69 (20), pp. 8141-8149. DOI: 10.1158/0008-5472.CAN-09-0919.

Walton, T. J.; Li, G.; Seth, R.; McArdle, S. E.; Bishop, M. C.; Rees, R. C. (2008): DNA demethylation and histone deacetylation inhibition co-operate to re-express estrogen receptor beta and induce apoptosis in prostate cancer cell-lines. In The Prostate 68 (2), pp. 210-222. DOI: 10.1002/pros.20673. 
Wan, Xinhai; Liu, Jie; Lu, Jing-Fang; Tzelepi, Vassiliki; Yang, Jun; Starbuck, Michael W. et al. (2012): Activation of beta-catenin signaling in androgen receptor-negative prostate cancer cells. In Clinical cancer research : an official journal of the American Association for Cancer Research 18 (3), pp. 726-736. DOI: 10.1158/1078-0432.CCR-11-2521.

Wang, Jun; Eltoum, Isam-Eldin; Lamartiniere, Coral A. (2004): Genistein alters growth factor signaling in transgenic prostate model (TRAMP). In Molecular and cellular endocrinology 219 (1-2), pp. 171-180. DOI: 10.1016/j.mce.2003.12.018.

Wang, Jun; Eltoum, Isam-Eldin; Lamartiniere, Coral A. (2007): Genistein chemoprevention of prostate cancer in TRAMP mice. In Journal of carcinogenesis 6, p. 3. DOI: 10.1186/1477-3163-6-3.

Wang, Qianben; Li, Wei; Zhang, Yong; Yuan, Xin; Xu, Kexin; Yu, Jindan et al. (2009): Androgen receptor regulates a distinct transcription program in androgen-independent prostate cancer. In Cell 138 (2), pp. 245-256. DOI: 10.1016/j.cell.2009.04.056.

Way, J. L. (1984): Cyanide intoxication and its mechanism of antagonism. In Annual review of pharmacology and toxicology 24, pp. 451-481. DOI: 10.1146/annurev.pa.24.040184.002315.

Wilcox, Cathy B.; Feddes, Grace O.; Willett-Brozick, Joan E.; Hsu, Lih-Ching; DeLoia, Julie A.; Baysal, Bora E. (2007): Coordinate up-regulation of TMEM97 and cholesterol biosynthesis genes in normal ovarian surface epithelial cells treated with progesterone: implications for pathogenesis of ovarian cancer. In BMC cancer 7, p. 223. DOI: 10.1186/1471-2407-7-223.

Williams, C.; Edvardsson, K.; Lewandowski, S. A.; Strom, A.; Gustafsson, J-A (2008): A genome-wide study of the repressive effects of estrogen receptor beta on estrogen receptor alpha signaling in breast cancer cells. In Oncogene 27 (7), pp. 1019-1032. DOI: $10.1038 /$ sj.onc.1210712.

Wiman, K. G. (2013): p53 talks to PARP: the increasing complexity of p53-induced cell death. In Cell death and differentiation 20 (11), pp. 1438-1439. DOI: 10.1038/cdd.2013.111.

Winn, Louise M.; Kim, Perry M.; Nickoloff, Jac A. (2003): Oxidative stress-induced homologous recombination as a novel mechanism for phenytoin-initiated toxicity. In The Journal of pharmacology and experimental therapeutics 306 (2), pp. 523-527. DOI: 10.1124/jpet.103.052639.

Witt, D. 2012. Analyse zur Rolle von pflanzlichen Wirkstoffen und HistondeacetylaseInhibitoren auf Wachstumsfaktoren und deren Signalwege in Prostatakarzinomzellen. Dissertation. University Göttingen, Germany. Institute for Human Genetics. 
Wong, Hao Yun; Burghoorn, Jan A.; van Leeuwen, Marije; Ruiter, Petra E. de; Schippers, Esther; Blok, Leen J. et al. (2004): Phosphorylation of androgen receptor isoforms. In The Biochemical journal 383 (Pt 2), pp. 267-276. DOI: 10.1042/BJ20040683.

Wu, L.; Runkle, C.; Jin, H-J; Yu, J.; Li, J.; Yang, X. et al. (2014): CCN3/NOV gene expression in human prostate cancer is directly suppressed by the androgen receptor. In Oncogene 33 (4), pp. 504-513. DOI: 10.1038/onc.2012.602.

www.akdae.de/Arzneimittelsicherheit/Bekanntgaben/Archiv/2014/20141212.html 29.11.2016 www.cancer.org/cancer/prostatecancer/detailedguide/prostate-cancer-treating-general-info 29.11.2016

www.illumina.com/products/scriptseq-rna-seq-library-prep.html 29.11.2016 www.krebsinformationsdienst.de/aktuelles/2014/news95.php 29.11.2016 www.promega.com/ /media/Files/Resources/Protocols/Technical\%20Manuals/101/ApoToxGlo\%20Triplex\%20Assay\%20Protocol.pdf 29.11.2016

www.wcrf.org/ 29.11.2016

Xu, Zhou; Fallet, Emilie; Paoletti, Camille; Fehrmann, Steffen; Charvin, Gilles; Teixeira, Maria Teresa (2015): Two routes to senescence revealed by real-time analysis of telomerase-negative single lineages. In Nature communications 6, p. 7680. DOI: $10.1038 /$ ncomms 8680 .

Yang, Cheng; Fischer-Keso, Regina; Schlechter, Tanja; Strobel, Philipp; Marx, Alexander; Hofmann, Ilse (2015): Plakophilin 1-deficient cells upregulate SPOCK1: implications for prostate cancer progression. In Tumour biology : the journal of the International Society for Oncodevelopmental Biology and Medicine 36 (12), pp. 9567-9577. DOI: 10.1007/s13277-015-3628-3.

Yang, Feng; Zhang, Yongyou; Ressler, Steven J.; Ittmann, Michael M.; Ayala, Gustavo E.; Dang, Truong D. et al. (2013): FGFR1 is essential for prostate cancer progression and metastasis. In Cancer research 73 (12), pp. 3716-3724. DOI: 10.1158/00085472.CAN-12-3274.

Yen, Wei-Lien; Klionsky, Daniel J. (2008): How to live long and prosper: autophagy, mitochondria, and aging. In Physiology (Bethesda, Md.) 23, pp. 248-262. DOI: 10.1152/physiol.00013.2008.

Yokoyama, Noriko N.; Shao, Shujuan; Hoang, Bang H.; Mercola, Dan; Zi, Xiaolin (2014): Wnt signaling in castration-resistant prostate cancer: implications for therapy. In American journal of clinical and experimental urology 2 (1), pp. 27-44. 
Zellweger, Tobias; Sturm, Susanne; Rey, Silvia; Zlobec, Inti; Gsponer, Joel R.; Rentsch, Cyrill A. et al. (2013): Estrogen receptor beta expression and androgen receptor phosphorylation correlate with a poor clinical outcome in hormone-naive prostate cancer and are elevated in castration-resistant disease. In Endocrine-related cancer 20 (3), pp. 403-413. DOI: 10.1530/ERC-12-0402.

Zhang, G. M.; Jin, B. Q. (1986): Pharmacokinetics of amygdalin in rabbits. In Zhongguo yao li $x$ ue bao = Acta pharmacologica Sinica 7 (5), pp. 460-462.

Zhang, Lei; Barritt, Gregory J. (2006): TRPM8 in prostate cancer cells: a potential diagnostic and prognostic marker with a secretory function? In Endocrine-related cancer 13 (1), pp. 27-38. DOI: 10.1677/erc.1.01093.

Zhu, Timothy C.; Finlay, Jarod C.; Hahn, Stephen M. (2005): Determination of the distribution of light, optical properties, drug concentration, and tissue oxygenation invivo in human prostate during motexafin lutetium-mediated photodynamic therapy. In Journal of photochemistry and photobiology. B, Biology 79 (3), pp. 231-241. DOI: 10.1016/j.jphotobiol.2004.09.013. 


\section{Acknowledgements}

I would like to acknowledge all the people involved in the generation of this thesis and supporting me during my time in the Institute for Human Genetics in Göttingen.

First, I would like to thank Prof. Dr. Peter Burfeind for giving me the opportunity to work on this interesting project. Thanks for your comments and for proofreading my manuscript. I learned a lot during my time in your lab.

I would like to thank the members of my thesis committee, Prof. Dr. Hubertus Jarry and Prof. Dr. Dieter Kube, for spending your time to help me improve my research, for your advice and ideas.

I was lucky to be part of many fruitful cooperations. Thanks to PD Dr. Paul Thelen, for allowing us to use his VCaP cell system and provide the $8 \beta$-VE2 and abiraterone actetate. Thanks to Prof. Dr. Roman Blaheta for introducing us to his amygdalin project and supporting our work financially and with his knowledge. Special thanks to Dr. Felix Bremmer for the analysis and staging of the tumor sections. Thanks to Dr. Michael Müller for performing the cyanide measurements and to Dr. Thomas Lingner and Dr. Andreas Leha, for helping me with the analysis of the whole mRNA-sequencing data. I appreciated the patience you showed answering all my questions. I also want to express my gratitude to the employees of the animal facility for doing a great job, guaranteeing the well-being of the mice used in this study.

My special thanks to PD Dr. Silke Kaulfuß. Thanks for your comments and for proofreading my manuscript. Thank you for encouraging me to follow my own ideas, for your critical feedback and for standing up for me.

During my PhD, I was grateful being part of a wonderful working group. Thanks to Lisa, Rabea, Laura, Amanda, Michelle, Britta, Jasmin, Sascha, Rovena, Claudia, Tashina, Tabea, Daria and Henning. Thanks for being such a great team and for the amicable atmosphere. It was great to talk, laugh and work with you. Special thanks to Lisa for being always cheerful and optimistic, for your help and your open ears and for caring for all of us. Thanks to Amanda, hardworking as you were you helped me lots with the amygdalin project.

I would furthermore like to thank my family and friends. Thanks for encouraging me to complete my thesis but even more for being part of my life. I am thankful to my parents for listening to my concerns, supporting me in all situations and helping me to find my way. I could not have any better parents than you.

Especially I would like to thank my husband Daniel for being there whenever I needed to talk, for caring and encouraging me when I was tired and for cooking dinner and doing the dishes. You always keep on telling me how proud you are. Thank you. 


\section{Curriculum Vitae}

Personal information

Name:

Julia Gehrig, née Meyer

Date of birth:

13.07.1988

Place of birth:

Hamelin

Nationality:

German

\section{Course of education}

1994-1998

1998-2000

2000-2007

2008-2011

$2011-2013$

2013-2016

Elementary school Pestalozzi, Hamelin

Orientierungsstufe-Nord, Hamelin

Albert-Einstein-Gymnasium, Hamelin,

Graduation: Abitur

Georg-August-Universität Göttingen

Studies in Biology

Graduation: BSc Biology

Georg-August-Universität Göttingen

Studies in Developmental, Neural and Behavioral

Biology

Graduation: MSc Biology

Georg-August-Universität Göttingen

Studies in the Ph.D. Program Molecular Medicine

Work-related internships

03.2012-04.2012

Wissenschaftliche Hilfskraft, Georg-

August-Universität Göttingen

Language skills

Englisch (UNIcert III Scientific English)

Göttingen, den 20.11.2016 